Nanostructured imaging surface plasmon resonance biosensing

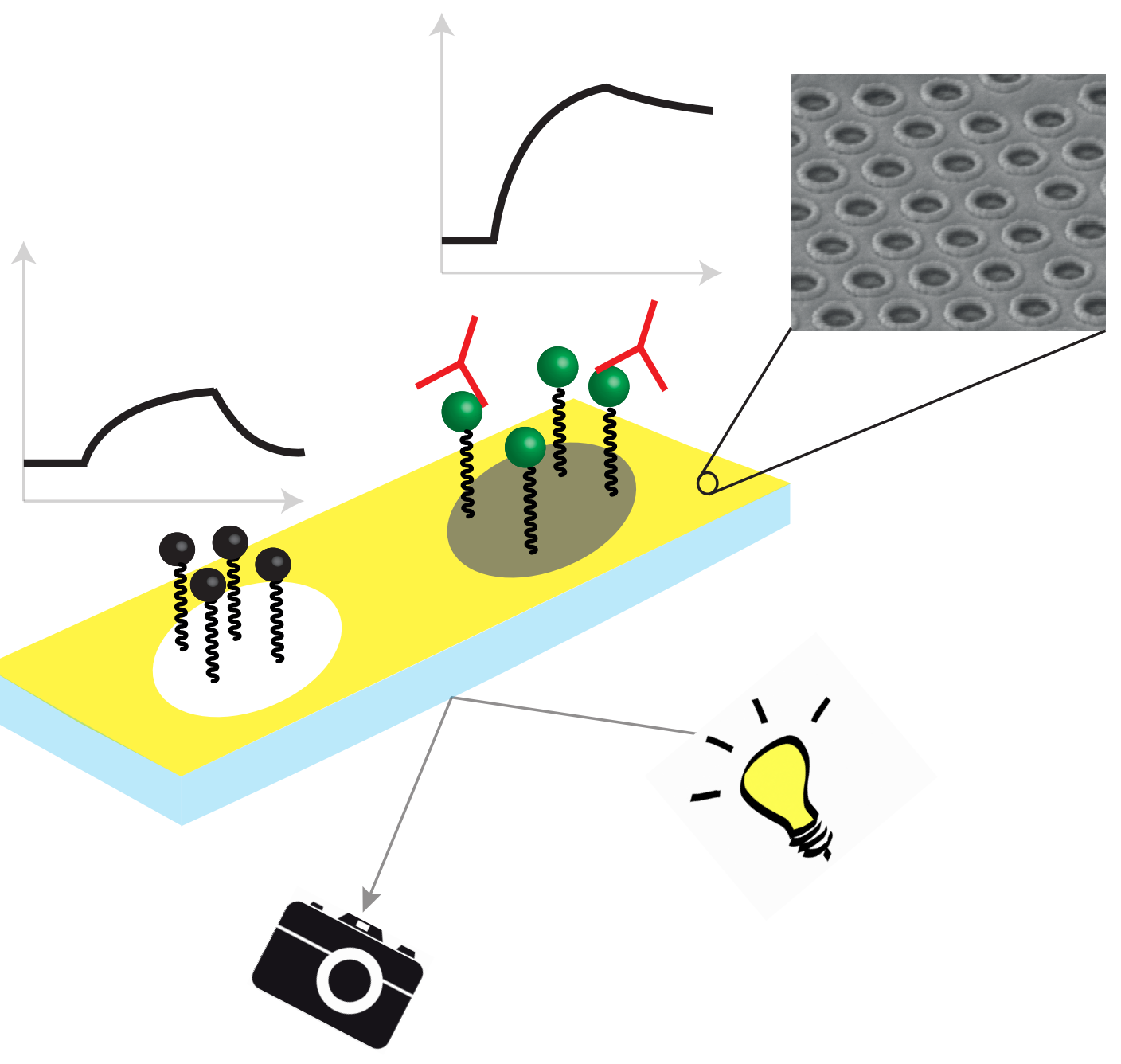

Sweccha Joshi 


\section{Propositions}

1. Demands of multiplex mycotoxin iSPR assays are not compatible with the wide range of legal limits for mycotoxins.

(this thesis)

2. Despite the progress made in antifouling chemistries, the one to beat carboxymethylated dextran in terms of SPR performance is still to be found.

(this thesis)

3. Simplicity comes at the cost of sensitivity.

4. NOED or NOESY NMR spectra can lead to wrong conclusions regarding the relative stereochemistry of natural products.

T. C. Fleischer et al. J. Nat. Prod. 1997, 60, 1054.

P. Weyerstahl et al. Flavour Fragr. J. 2000, 15, 153.

A. A. Stierle et al. J. Nat. Prod. 2003, 66, 1097.

K. I. Booker-Milburn et al. Org. Lett. 2003, 5, 3309.

S. Amand et al. J. Nat. Prod. 2012, 75, 798.

5. Small talk in the Netherlands would not be complete without discussing the weather.

6. Specifications of electric cars claiming zero $\mathrm{CO}_{2}$ emission per $\mathrm{km}$ reflect the attempt of the industry to satisfy unrealistic expectations of consumers.

Propositions belonging to the thesis, entitled

'Nanostructured imaging surface plasmon resonance biosensing'

Sweccha Joshi

Wageningen, 10 February 2017 


\section{Nanostructured imaging surface plasmon resonance biosensing}

Sweccha Joshi 


\section{Thesis committee}

\section{Promotors}

Prof. Dr M.W.F. Nielen

Professor of Analytical Chemistry, with special emphasis for the detection of chemical food contaminants

Wageningen University

Prof. Dr H. Zuilhof

Professor of Organic Chemistry

Wageningen University

\section{Co-promotor}

Dr T.A. van Beek

Assistant Professor, Laboratory of Organic Chemistry

Wageningen University

\section{Other members}

Prof. Dr M.H.M. Eppink, Wageningen University

Prof. Dr M.W.J. Prins, Eindhoven University of Technology

Prof. Dr G.W. Somsen, VU Amsterdam

Dr T. Kudernac, University of Twente

This research was conducted under the auspices of the Graduate School VLAG (Advanced Studies in Food Technology, Agrobiotechnology, Nutrition and Health Sciences). 


\title{
Nanostructured imaging surface plasmon resonance biosensing
}

Sweccha Joshi

\author{
Thesis \\ submitted in fulfilment of the requirements for the degree of doctor \\ at Wageningen University \\ by the authority of the Rector Magnificus \\ Prof. Dr A.P.J. Mol, \\ in the presence of the \\ Thesis Committee appointed by the Academic Board \\ to be defended in public \\ on Friday 10 February 2017 \\ at 4 p.m. in the Aula.
}


Sweccha Joshi

Nanostructured imaging surface plasmon resonance biosensing, 164 pages.

PhD thesis, Wageningen University, Wageningen, NL (2017)

With references, with summary in English

ISBN 978-94-6343-020-3

DOI $10.18174 / 398439$ 


\section{Table of Contents}

\section{List of abbreviations}

Chapter 1 General introduction

Chapter 2 Surface characterization and antifouling properties of nanostructured gold chips for imaging surface plasmon resonance biosensing

Chapter 3 Multiplex surface plasmon resonance biosensing and its transferability towards imaging nanoplasmonics for detection of mycotoxins in barley

Chapter 4 Analysis of mycotoxins in beer using a portable nanostructured imaging surface plasmon resonance biosensor

Chapter 5 Biochip Spray: Simplified coupling of surface plasmon resonance biosensing and mass spectrometry

Chapter 6 General discussion and future perspectives

Summary 



\section{To my mother Veena Sharma}

"It does not matter how slowly you go as long as you do not stop" Confucius 



\section{List of Abbreviations}

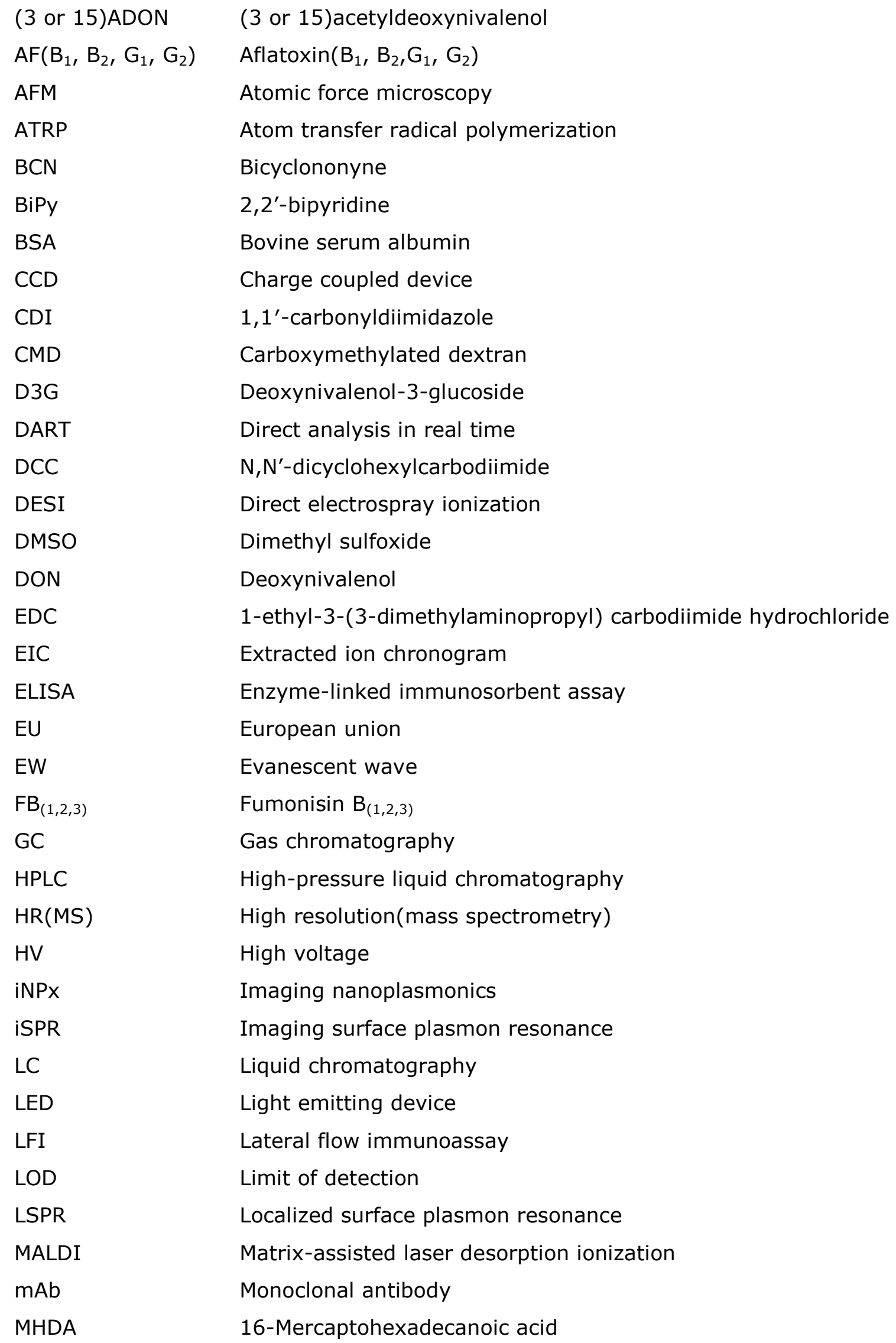




\begin{tabular}{|c|c|}
\hline ML & Maximum level \\
\hline NHS & N-hydroxysuccinimide \\
\hline NP & Nanoparticles \\
\hline OEG & Oligo(ethylene glycol) \\
\hline OT $(A, B)$ & Ochratoxin $(A, B)$ \\
\hline OVA & Ovalbumin \\
\hline PE-CVD & Plasma-enhanced chemical vapor deposition \\
\hline PEG & Poly(ethylene glycol) \\
\hline PEG1000 and & Poly(ethylene glycol) 2-aminoethyl ether acetic acid (average MW 1000 \\
\hline PEG3500 & and 3500) \\
\hline PEGMA & Poly(ethylene glycol) methacrylate \\
\hline PFP & Pentafluorophenol \\
\hline PMMA & Poly(methyl methacrylate) \\
\hline PS & Polystyrene \\
\hline ROI & Region of interest \\
\hline RSD & Relative standard deviation \\
\hline SAM & Self-assembled monolayers \\
\hline SAMDI & Self-assembled monolayers laser desorption/ionization \\
\hline SBMA & 2-(methacryloyloxy)ethyl)dimethyl-3-sulfopropyl)ammonium hydroxide \\
\hline SELDI & Surface-enhanced laser desorption ionization \\
\hline SEM & Scanning electron microscopy \\
\hline SERS & Surface enhanced raman spectroscopy \\
\hline SI-ATRP & Surface initiated atom transfer radical polymerization \\
\hline SP(M)E & Solid phase (micro) extraction \\
\hline SPR & Surface plasmon resonance \\
\hline TDI & Tolerable daily intake \\
\hline TIC & Total ion chronogram \\
\hline TSL & Theoretical safe limit \\
\hline WCA & Water contact angle \\
\hline XPS & X-ray photoelectron spectroscopy \\
\hline ZEA or ZEN & Zearalenone \\
\hline$a-Z E L$ & a-zearalanol \\
\hline
\end{tabular}




\section{Chapter 1}

General Introduction 


\section{Surface plasmon resonance based label free biosensors}

The combination of the optical detection technique surface plasmon resonance (SPR) and biosensing has given rise to the field of SPR-based biosensors. ${ }^{1-3}$ SPR-based biosensors have received considerable attention in the past decades as they allow fast, reliable and label-free detection of analytes. SPR, in addition, benefits from real-time monitoring of the interaction kinetics and reusability of the biosensor chip. The current interest in the field of biosensing has been on portable devices to bring the lab to the sample. However, most SPR instruments are laboratory based and do not fulfill this requirement. Therefore, there is a high demand for a portable SPR instrument that would allow detection of multiple analytes directly in the field. In the next few sections, background information about SPR, SPR instruments along with their components, development of a multiplex SPR biosensor and coupling of SPR to mass spectrometry is described.

\section{Surface plasmon resonance (SPR)}

When a monochromatic plane polarized light (electric vector component is parallel to the interface) passes through a prism and strikes the glass side of an SPR chip (Figure 1A) under conditions of total internal reflection, an electric field known as an evanescent wave (EW) is generated. ${ }^{4}$ The EW propagates along the interface and its amplitude decreases with increasing distance from the interface. The EW is absorbed by the free electron clouds in the gold layer (on the other side of the chip) to generate electron charge density waves called surface plasmons (SPs). As some of the incident light is absorbed, the intensity of the reflected light decreases as a function of the angle of reflection (Figure $1 B$ ). The angle at which the intensity of the reflected light is minimal is called the resonance angle or SPR angle (Figure 1B). This SPR angle is sensitive to the refractive index of the fluid close to the gold side and changes (Figure 1B, change from I to II) upon a binding event at the surface thus allowing label-free detection of analytes. The SPR angles are used as response and plotted against time in a graph showing the real-time change in SPR response called a sensorgram (Figure $1 \mathrm{C}$ ). In addition to the information about the amount of analyte binding to the surface, kinetic data can also be extracted from these sensorgrams. The kinetic data gives information about the rate of association or dissociation and strength of the overall interaction.

\section{Imaging SPR}

Imaging surface plasmon resonance (iSPR) ${ }^{5,6}$ is a variation of SPR that allows detection of multiple analytes on a single chip as the intensity of light coming from the entire chip is collected (by a CCD camera) as a signal (Figure $2 \mathrm{~A}$ ). The change in intensity of light is used as the SPR response (Figure 2A, I-III). The so-called regions of interest (ROIs, 
white circles in Figure $2 \mathrm{~A}$ ) can be used as detection areas, allowing analysis of few analytes up to an entire array (for high-throughput screening) depending on the application. $^{7}$

A
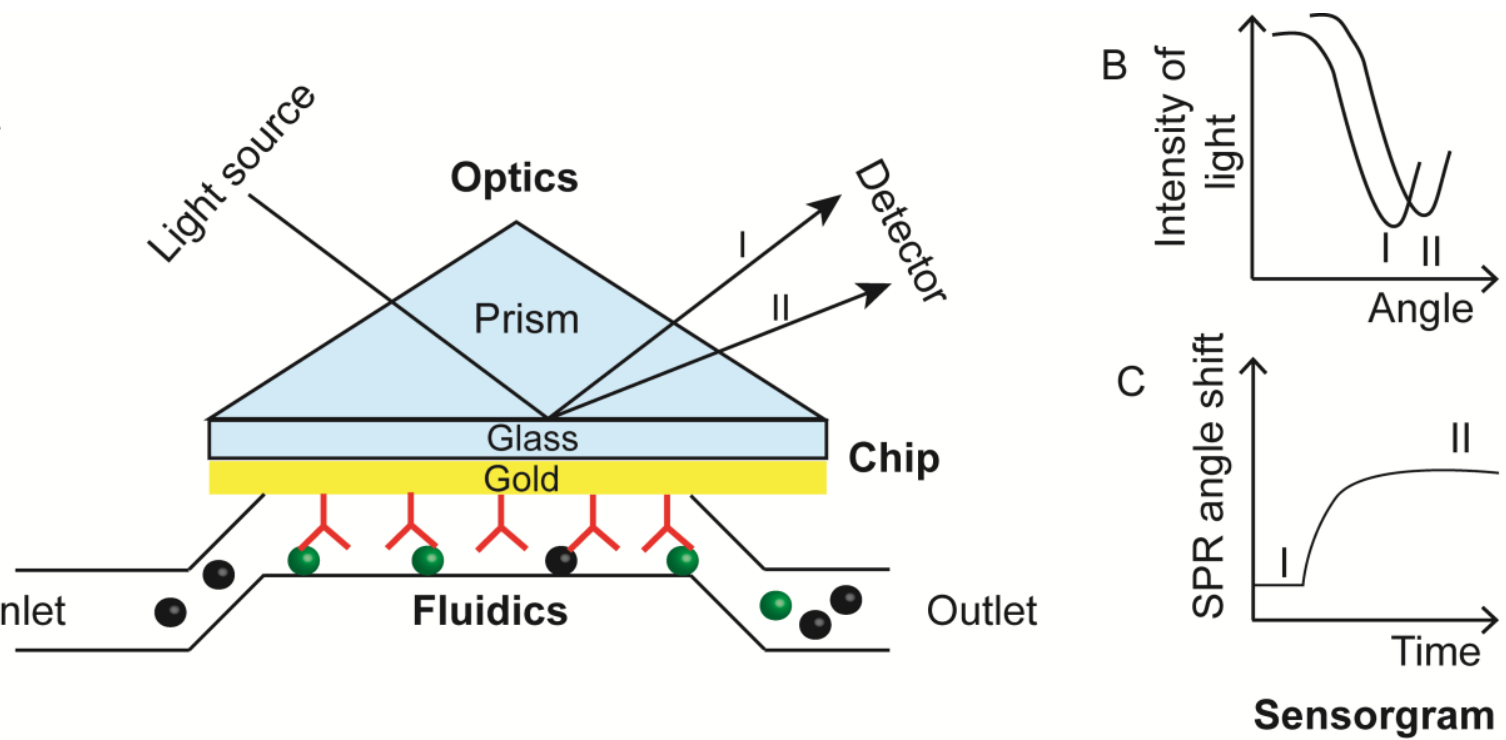

Figure 1. A) An SPR setup in Kretschmann configuration consisting of an optical part (light source, prism and detector), sensor chip and a liquid handling system (fluidics). B) The angle-dependent intensity, and the shift thereof (from I to II) upon a binding event at the surface. C) Translation of the SPR angle shift into a real-time change in SPR response (sensorgram).

\section{Localized SPR}

Localized surface plasmon resonance (LSPR) is a phenomenon that occurs when light interacts with particles much smaller than the incident wavelength thus creating a plasmon that oscillates locally around the particle with a frequency known as the LSPR frequency band (Figure 2B). ${ }^{8}$ Localized surface plasmons can have a shorter decay length (e.g. 6 nm) compared to normal SPR ( 200 nm), which makes LSPR-based biosensors more sensitive to changes in the local refractive index. ${ }^{9-11}$ The resulting small sensing volume (region where refractive index-based sensing can occur) significantly reduces the effect of bulk change of refractive index, and can be advantageous for the detection of small molecules. 


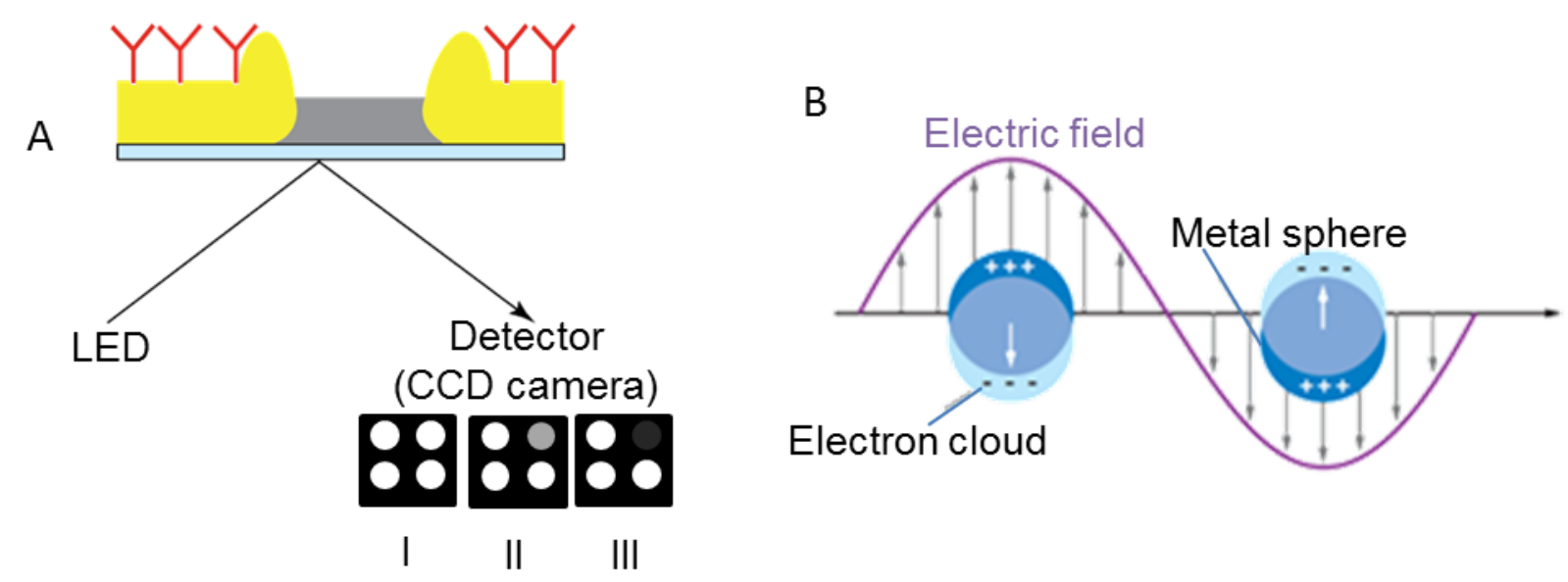

Figure 2. Schematic representation of A) an imaging SPR setup with antibodies immobilized on the surface, where the change in intensity of reflected light (I-III) upon binding of an analyte is recorded using a CCD camera. The highest intensity (I) is recorded when no analyte is present and intensity decreases (II and III) in a specific ROI (top right with antibodies against a specific analyte) as binding occurs while no change is seen in the other three ROIs (antibodies against other analytes or reference). B) Schematic illustration of a localized surface plasmon where the electric field generated around metal nanoparticles is depicted (reproduced with permission from ref. 8).

\section{Prismless SPR detection}

Traditional SPR uses glass coated on one side with flat gold as sensor surface. Such a setup requires a prism for coupling of the wavevector of the incident light to that of the surface plasmons. More recently, along with advancements in nanotechnology, nanostructured surfaces have emerged as an alternative to flat gold surfaces. ${ }^{12}$ One example of such a nanostructured surface consists of a dielectric substrate with a periodic, sub-wavelength nanohole array drilled (e.g., by ion beams) into the gold layer (Figure 3A, inset). ${ }^{13-15}$ In line with this new technology, a novel nanostructured chip consisting of periodic alternation of PMMA wells in optically thick gold films was developed. ${ }^{16}$ In both cases, the nanoarray acts as the metal dielectric required for the coupling of light to the surface plasmons, thus omitting the use of prisms. The periodic alternation of holes/wells with metallic film gives rise to localized plasmonic resonances, which in combination with the normal surface plasmonic resonances can lead to higher sensitivity. ${ }^{17}$ Unlike the nanoholes where the maximum of the electric field (maximum sensitivity) is along the walls of the holes (Figure $3 \mathrm{~B}),{ }^{18}$ the maximum in case of the nanostructured gold chip is at the top of the PMMA wells (Figure $3 \mathrm{C}$ ). ${ }^{19}$ This makes the latter more suitable for immobilization of bio-receptors in the zone with maximum sensitivity as the top of the wells are more accessible than the walls of the holes. 
A

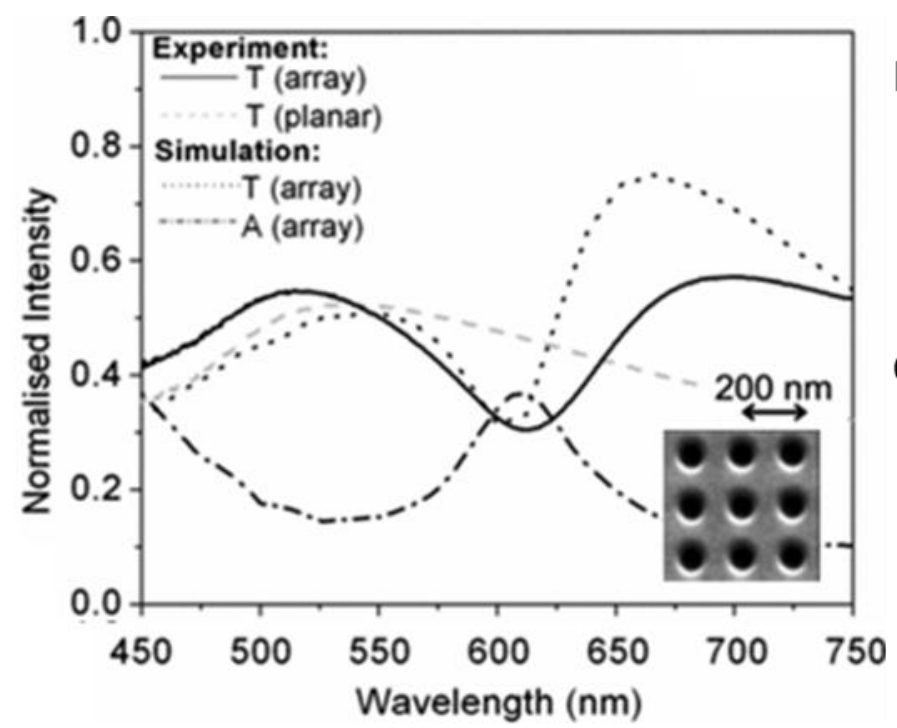

B

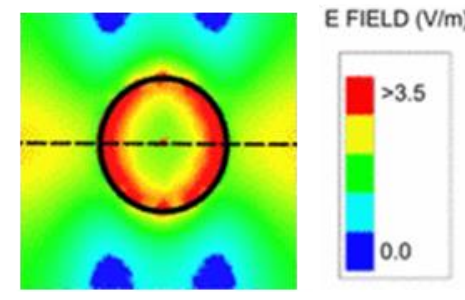

C

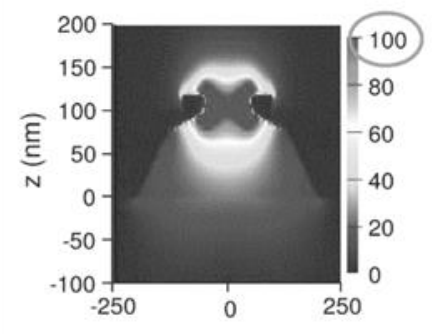

Figure 3. A) Experimental transmittance spectra ( $T$ array) compared to simulated transmittance and absorbance spectra (A array) for a nanohole array (inset) with $90 \mathrm{~nm}$ diameter holes in a 20 $\mathrm{nm}$ thick gold film (periodicity of $200 \mathrm{~nm}$ ), illuminated at normal incidence in air. The grey dashed line shows the transmittance spectra for a $20 \mathrm{~nm}$ flat gold film (T planar). A minimum observed around $600 \mathrm{~nm}$ in case of the array is due to the excitation of the LSPR. B) Electric field distribution for the nanohole array (reproduced with permission from ref. 18). C) Electric field distribution for the nanostructured gold (reproduced with permission from ref. 19).

\section{SPR instruments}

The SPR instrument industry is dominated by Biacore (Uppsala, Sweden). ${ }^{20}$ Biacore 3000 (Figure 4A), the most successful model from the manufacturers, allows detection of three different biointeractions in parallel plus a reference. Nevertheless, several other SPR instruments have been developed over the years all occupying different niches. ${ }^{21}$ Amongst the imaging SPR instruments, IBIS $^{22}$ (Figure 4B) has developed a model that has been very successfully applied for multi-analyte screening for food safety ${ }^{23}$ as well as medical applications. ${ }^{24,25}$ Both instruments (Biacore and IBIS) are benchtop and have been used as benchmark instruments in this thesis.
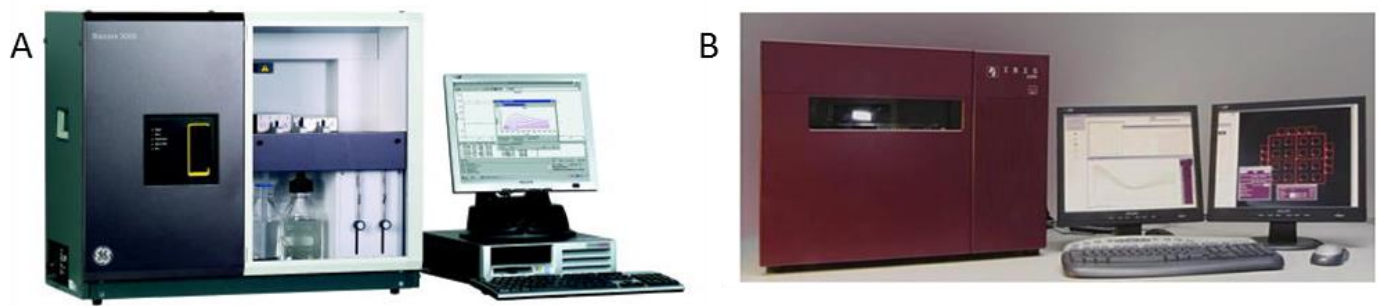

Figure 4. A conventional benchtop A) SPR (Biacore 3000) and B) imaging SPR (IBIS) instrument. Images from www.gelifesciences.com and www.ibis-spr.nl respectively. 
There is a clear demand for simple and affordable instruments preferably in a portable format in the field of SPR. Several miniaturized SPR instruments, ranging from smaller benchtop to smartphone-based models, have been developed over the past years. ${ }^{26-32}$ However, there is a lack of a detailed study or a comparison with the benchmark instruments. ${ }^{33}$ The aim of this thesis is basically to fill this gap. To this aim, to properly analyze the potential and limitations of such portable SPR instruments, a recently developed nanostructured iSPR, 14 times smaller, 8-11 times lighter and 7-12 times cheaper than the commercial SPR (Biacore 3000) and iSPR (IBIS), was chosen for detailed study in this thesis. The instrument integrates optics, liquid handling and data analysis in a compact, stand-alone and portable format (Figure 5).

A

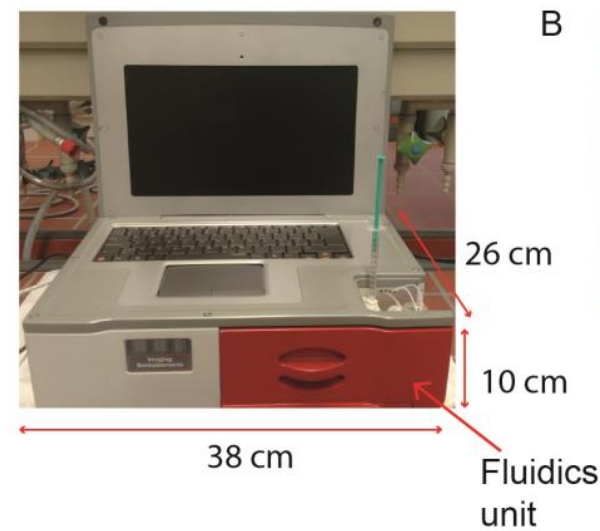

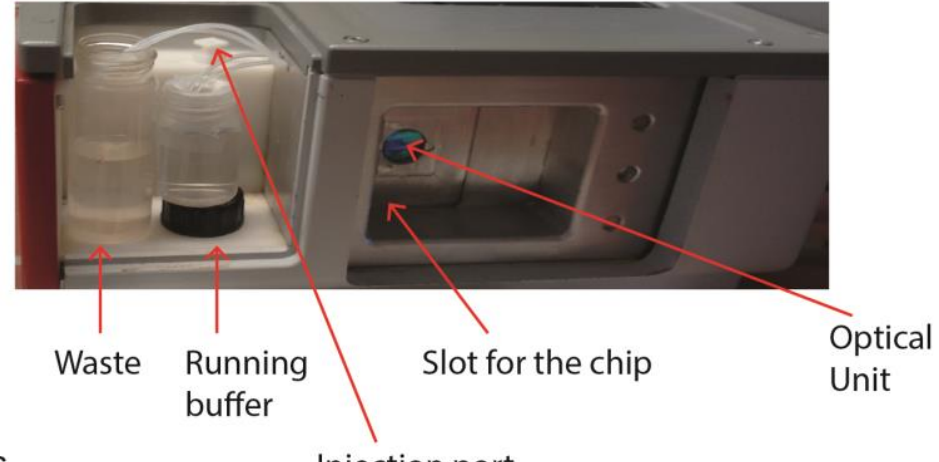

Injection port

Figure 5. A) The nanostructured imaging SPR instrument, also referred to as imaging nanoplasmonics. The fluidics unit can be removed. B) View of the instrument from the right side where the running buffer and waste container can be placed. The sample can be injected manually via the injection port using a syringe. The chip can be installed in the slot such that it is in contact with the fluidics and the glass side is facing the optical unit.

\section{Components of an SPR instrument}

A typical SPR biosensor setup consists of a sensor chip, an optical unit (light source, detector and optical couplers), a liquid handling system and a computing unit. The details of each component depend on the instrument and are described below for the three instruments used in the work described in this thesis. An (i)SPR sensor chip consists of a chip with a glass side (high refractive index) and a metal side (low refractive index) to form the metal dielectric interface. In all three instruments (Biacore, IBIS and imaging nanoplasmonics), the glass side of the sensor chip is in contact with the optical part while the metal is in contact with the fluidics. Gold is mostly used as metal as it produces a strong SPR response, is inert, and can be easily functionalized using thiol chemistry for further immobilization of biomolecules. ${ }^{34}$ Other metals such as silver, aluminum, titanium, copper or chromium have been explored, but only to a limited extent. ${ }^{35}$ In both 
Biacore and IBIS, a flat gold surface is used, whereas imaging nanoplasmonics uses a nanostructured iSPR chip.

The optical part of an SPR consists of a light source for the incident light that gets coupled to the surface plasmons via the optical coupler and is reflected back and collected by a detector. The SPR detection can be performed at fixed angle, fixed wavelength or fixed angle and wavelength. In the former two cases, the change in the second variable (wavelength or angle) is monitored whereas in the last case, the change in intensity of the light is measured as a function of time. Both Biacore and IBIS are based on the Kretschman configuration and use light sources of fixed wavelength of 760 $\mathrm{nm}$ (Biacore) and $840 \mathrm{~nm}$ (IBIS). In the Biacore, the change in the SPR angle upon a binding event at the surface is used as the SPR response. Imaging SPR instruments, however, measure the change in intensity of reflected light at a fixed wavelength. The conventional iSPR (IBIS) uses a fixed wavelength light source and additionally allows scanning of optical angle (range $8^{\circ}$ ) to find the optimal SPR angle. In contrast to both the conventional instruments, the portable iSPR setup consists of two light sources one with a wavelength of $850 \mathrm{~nm}$ and the other of $940 \mathrm{~nm}$. The change in intensity of the reflected light at a fixed wavelength and angle is monitored. An optical coupler is required for matching of the wave vector of the surface plasmons with that of the incident light. In most conventional SPR instruments, including Biacore and IBIS, prism couplers are used. In prism-based systems, refractive index matching between the sensor chip and prism is required. This can be quite tedious in case of some instruments, such as the IBIS, where the sensor chip has to be removed and the prism has to be cleaned manually. An alternative where the prism is coated with gold has been introduced but this increases the cost of the analysis. Some instruments have attempted to overcome this problem by using grating couplers that use metallic grating as optical couplers (Figure 6A). ${ }^{36,37}$ Unlike in a prism-coupled SPR setup, the light passes through the sample in case of grating couplers. This is often the reason for lower and unstable signals obtained for grating couplers in comparison to prism couplers. From this point of view, the imaging nanoplasmonics is superior to both prism and grating couplers. Firstly, the nanostructured gold surface used in the portable instrument acts as a nanograting. This can replace the prism that is found in traditional SPR setups, thus contributing to the miniaturization of the instrument. This has helped to minimize the optical component, reduce the cost by omitting expensive prisms and made the setup including chip mounting simpler. Secondly, unlike grating couplers, the light does not pass through the sample thus overcoming the traditional problems of grating couplers. It has been proven that, in case of nanostructured gold, the reflectance measurement from the glass side of the chip is sensitive to the change in refractive index on the gold side. ${ }^{38}$ Fiber optics is the third type of optical couplers that can be used. ${ }^{39}$ The silicon from a small portion of 
the fiber is removed and coated with metal followed by a dielectric layer as shown in Figure 6B. Due to their low prices, they offer a disposable solution for medical applications. Furthermore, they do not contain any moving parts and have an advantage over the bulkier prism based sensors.

A

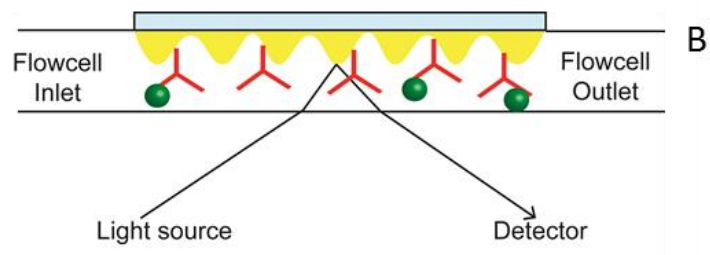

B

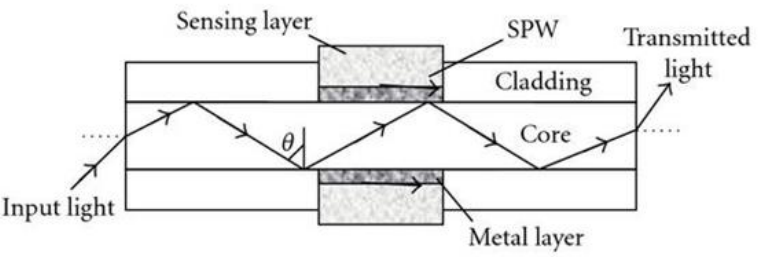

Figure 6. Schematic representation of a A) grating coupler and B) fiber optic coupler (reproduced with permission from ref. 39).

The fluidics of most SPR instruments consists of a flow-cell that allows continuous flow of the solutions. While Biacore and the portable iSPR instrument use a continuous flow system, a back and forth flow is used in IBIS instruments. Cuvette-based systems can also be found in some instruments. Cuvette systems are often preferred for analysis of samples such as blood and cell cultures, as clogging is less of a problem in such systems compared to flow-cell-based systems. However, efficient mixing of the sample is a big challenge in cuvette-based systems, thus making flow-cells more popular. Furthermore, there are also liquid handling systems, such as in Biacore 4000, that consist of four independent flow-cells, each containing five detection spots that improve the throughput of the system. Both the conventional iSPR (IBIS) and the portable iSPR consist of a single flow-cell. However, due to the imaging technology multiple detection spots can be incorporated. The detection spots, depending on the printing technology and the application, can range from a few spots to an entire array for high throughput applications.

Every SPR instrument is equipped with a computing unit that allows control of the instrument and software that allows data analysis. In case of Biacore, the entire process including docking of chip, sample injection and output data is automated. A similar control is possible with IBIS, except for the docking of the chip which is done manually. In case of imaging nanoplasmonics, the fluidics and optical unit are controlled using a software, while chip mounting and sample injection are done manually. The data obtained can be analyzed by dedicated software, such as Scrubber (BioLogic Software Pty Ltd., Campbell, Australia), Sprint (IBIS technologies B.V., Enschede, the Netherlands), ImageJ, etc., or can be exported into Excel for further analysis. 


\section{Nanostructured imaging surface plasmon resonance instrument}

Imaging nanoplasmonics uses a novel nanostructured gold surface as the sensor chip, which has several advantages in the overall setup as discussed above. The nanostructured gold chips were produced by Plasmore Srl. (Italy) using colloidal lithography and plasma-enhanced chemical vapor deposition (PE-CVD). As depicted schematically in Figure 7, poly(methyl methacrylate) (PMMA) is deposited on the glass substrate by PE-CVD using methyl methacrylate as liquid precursor. Spin coating is then used to cover the film with polystyrene (PS) beads (500 nm with a nominal size dispersion of $10 \%$ ). Next, the sample is exposed to oxygen plasma etching to remove the PMMA from the areas not covered by the beads and to reduce the size of the PS beads to provide the required periodicity. A gold layer of $100-200 \mathrm{~nm}$ is then deposited on the surface by physical vapor deposition. Finally, the polystyrene beads are removed, resulting in a surface with a gold film perforated by PMMA wells. The combined control of deposition and etching parameters allows fine tuning of film thickness and well diameters. The PMMA regions with diameters of 200-250 nm and periodicity (distance between the centers of the two PMMA wells) of 500-600 nm make up around $20 \%$ of the total surface area. Details about the modification and characterization of the nanostructured chip can be found in Chapter 2.

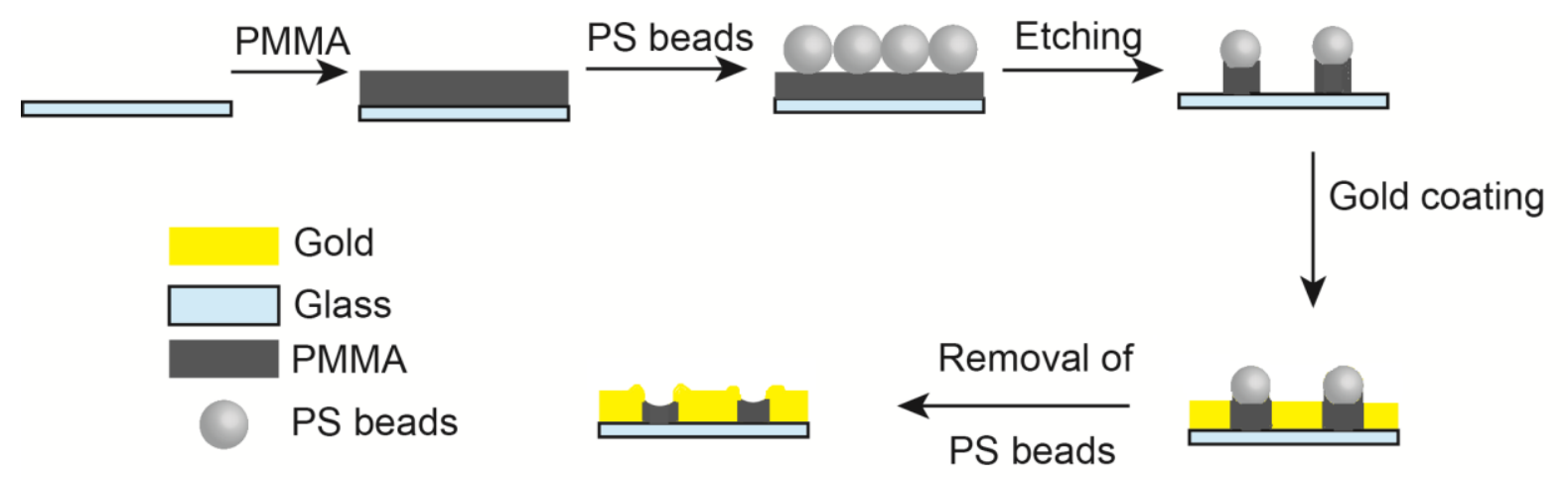

Figure 7. Schematic representation of the production of nanostructured iSPR chips.

The optical setup consists of two light-emitting devices (LEDs) as light source (test light source in Figure 8A), for excitation of SPR and obtaining of signal around 850 $\mathrm{nm}$ (LED1' in Figure 8B) and the other around $940 \mathrm{~nm}$ (LED1 in Figure 8B). As shown in Figure $8 \mathrm{~B}$, with increasing refractive index, the intensity of the reflected light decreases between 750-850 nm, whereas it increases above $900 \mathrm{~nm}$. Therefore, use of the two LEDs in alternation and combining the two signals allows improvement in sensitivity by increasing the total signal. Furthermore, as seen from Figure $8 \mathrm{~B}$, there is no change in intensity between 500-600 nm. A reference LED (LED2 in Figure 8B) emitting light in that range is used as reference light source (Figure $8 \mathrm{~A}$ ) to correct for any artefacts caused by 
temperature fluctuations or light source fluctuations. The incident light beams strike the chip at a fixed angle and the change in intensity of reflected light is recorded in reflectance mode. Unlike most conventional nanohole setups that work in transmittance mode ${ }^{14}$ there is no interference due to the sample or the nanostructure feature of the chip. A charge coupled device (CCD) is used as detector (Figure $8 \mathrm{~A}$ ) for the reflected light, as it allows spatial mapping of the entire biosensor chip, which is the core of the multiplexing ability of the instrument.

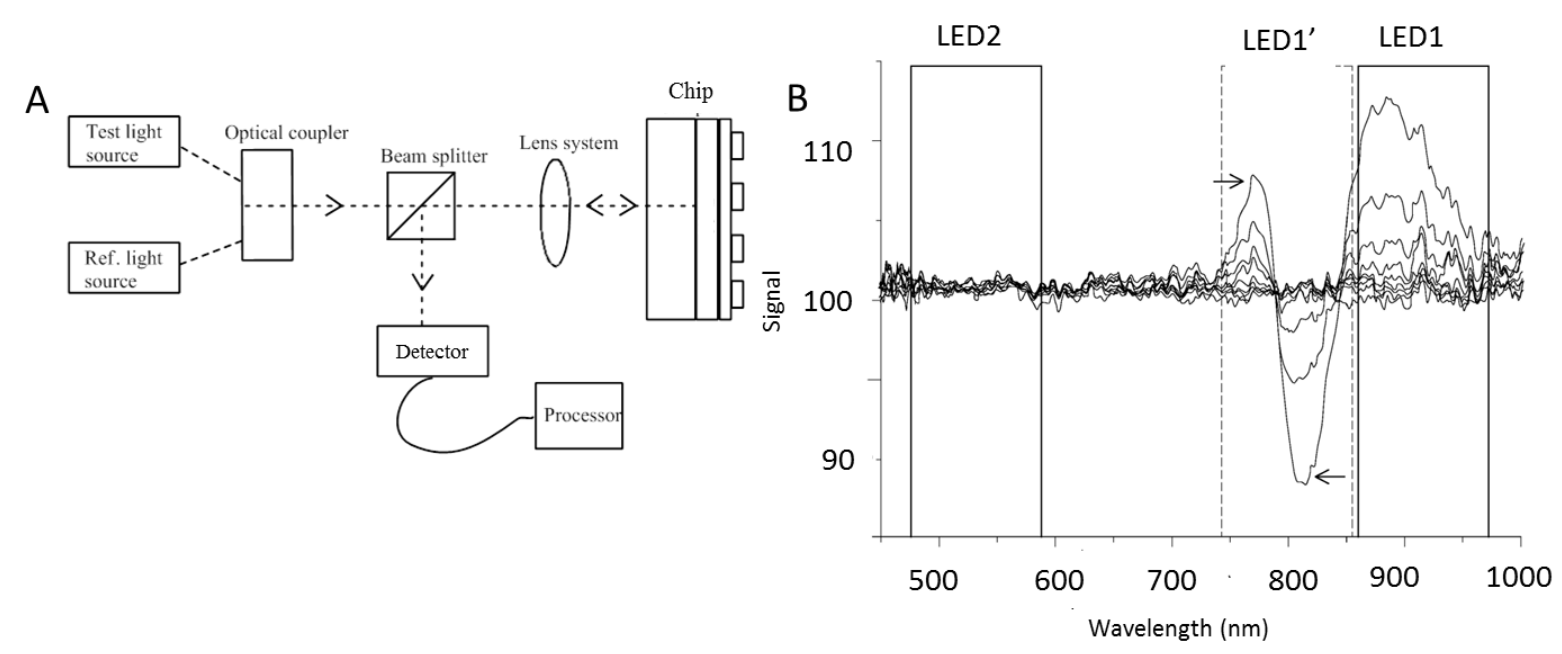

Figure 8. A) Schematic illustration of the preferred SPR optical setup and B) change in the spectra of the nanostructured chip recorded in reflectance mode upon increase in refractive index (reproduced with permission from reference 16).

The fluidics of the portable iSPR instrument (Figure 9) consists of two automatic syringe pumps (connected to the two inlet tubings). Each pump is connected to a 3-port valve, both connected to a T-piece, which ensures continuous flow of the buffer during the entire experiment by simultaneous switching. The chip is mounted on a flow-cell with a PDMS ring. The gold side of the chip is placed towards the ring (to ensure proper sealing) and fixed using a plastic window supported by six stainless steel pins. The flowcell can then be installed on the side of the instrument (Figure 5B) such that it is in contact with the fluidics and the glass side is facing the optical unit. The injection of the sample is controlled using a 6-port Rheodyne valve that can be loaded with sample via the injection port (in LOAD mode) and injected into the flow-cell by switching to INJECT mode with the help of the T-piece and 3-port valve. Any excess buffer or sample from the loop $(50 \mu \mathrm{L})$ is collected in the waste container. The dead volume of the entire fluidics is $70 \mu \mathrm{L}$, thus requiring at least $120 \mu \mathrm{L}$ of sample to completely fill the loop. The injection is performed manually, while the pumps and the switching of the Rheodyne valve can be controlled using the software. 


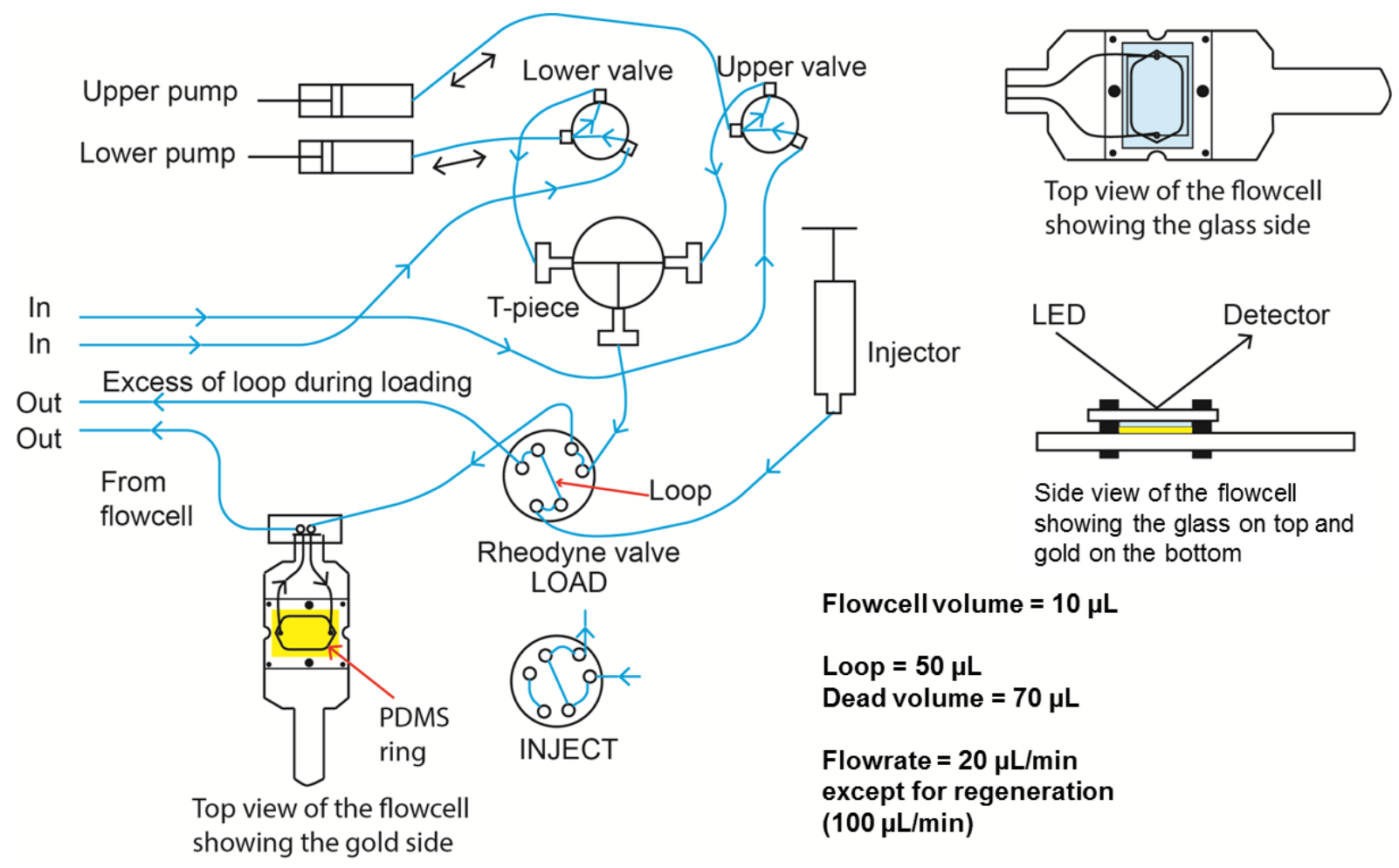

Figure 9. Schematic representation of the fluidics of the portable iSPR instrument. The chip is mounted onto a plastic flow-cell and sealed using a PDMS ring. The chip is placed such that its glass side faces the optical unit (LED and detector) and the gold side is in contact with the fluidics.

The imaging nanoplasmonics iSPR is operated using a computer (laptop size). The fluidics and optical unit is controlled via the software NanoPlasmonix Imaging V1 (Figure 10 , left side). Once, the chip is mounted onto the instrument, the live image can be visualized and the camera settings (exposure and gain) can be changed in such a way that a good contrast is obtained between the chip and the PDMS ring. The acquisition settings allow tuning of the lateral and time resolution (number of images per second) with three options: No decimation (highest lateral resolution; 1 image every $10 \mathrm{sec}$ ), 1/2 decimation (medium lateral resolution; 1 image every $6 \mathrm{sec}$ ) and 1/4 decimation (lowest lateral resolution; 1 image sec). As the instrument has two LEDs (850 nm and $940 \mathrm{~nm}$ ), they can be used individually or in alternation (strobe mode, the two responses can then be summed up). The ROI setup allows defining regions of interest (of different shape and size) including any reference ROIs. A reference outside the flow-cell is chosen to correct for any fluctuations of the light while a reference inside the flow-cell can help correct for any non-specific binding of the matrix. The ROIs, once saved can be loaded for later experiments and can be visualized in the upper right window of the software. The pump plus its speed and position of the Rheodyne valve can be controlled using the buttons on the lower left corner of the software. Once, all the settings have been chosen, the experiment can be started with the option to store the raw images as data. The first 
image of the experiment is taken as the reference image such that the difference image can be visualized and the corresponding sensorgram can be seen in the lower right corner. At the end of the experiment, an Excel file is generated containing the intensity of the raw images as well as the intensity after correcting for the reference outside.

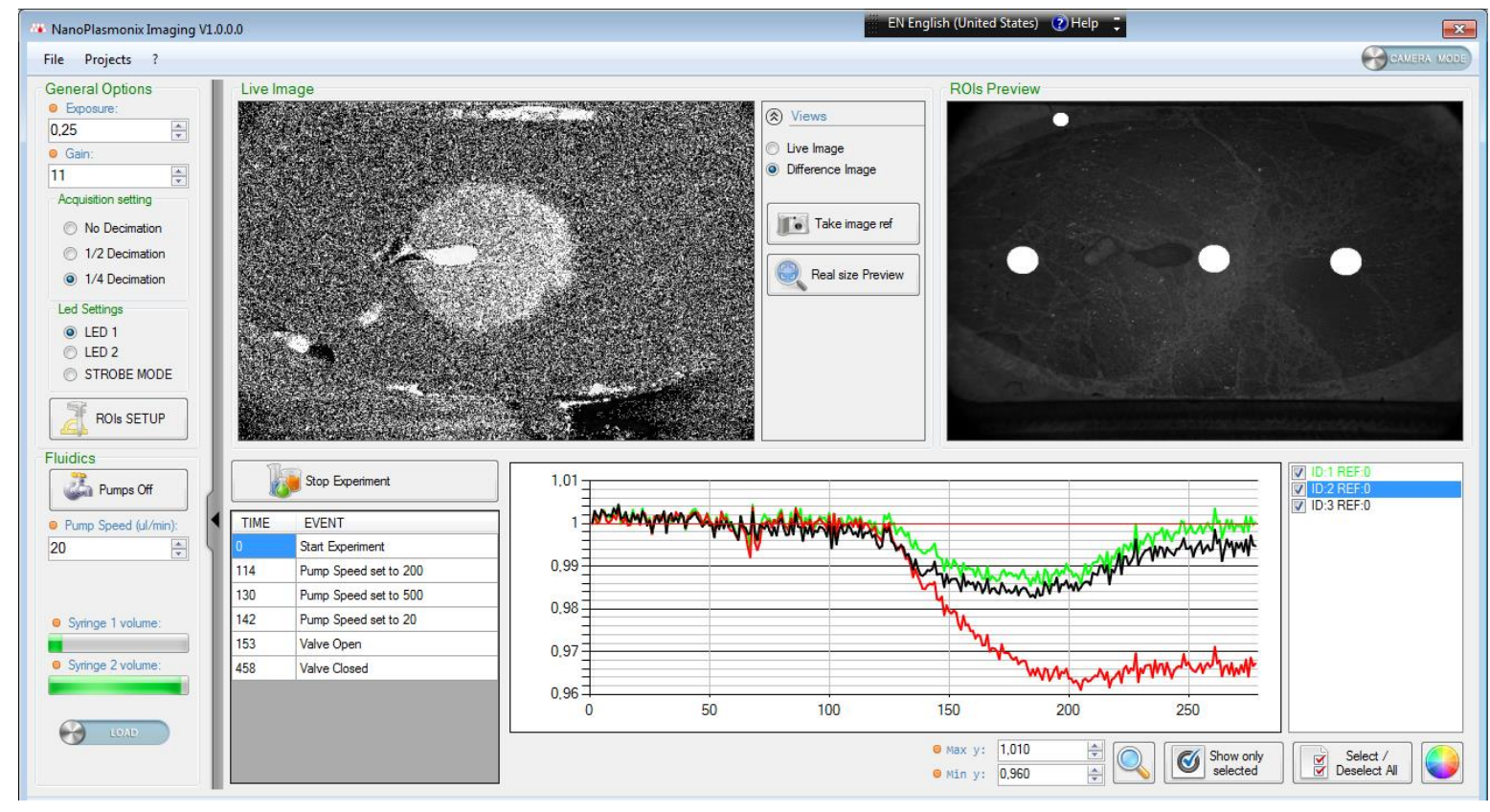

Figure 10. A screenshot of the PC screen (NanoPlasmonix Imaging V1 software) showing the controls for the camera, ROI setup and fluidics on the left side. The live or difference image and the ROI position can be seen on the top panels while the lower panels show the log of the experiment and sensorgrams obtained for each ROI. Upon binding of an analyte, although the image shows an increase in intensity as expected, the current software displays an opposite effect in the sensorgram. This is due to settings in the current software that needs to be updated but can be easily corrected in Excel during data processing.

\section{Nanostructured iSPR biosensing for the detection of multiple mycotoxins}

Mycotoxins are secondary metabolites of fungi known to be toxic to animals and humans. ${ }^{40}$ The most important mycotoxins are produced by the genera Aspergillus, Fusarium, Alternaria and Penicillium. ${ }^{41}$ In the European Union (EU), maximum levels $(M L s)^{42}$ are set for aflatoxins ( $A F B_{1}$ individually and summed with $A F B_{2}, A F G_{1}$ and $A F G_{2}$ ), deoxynivalenol (DON), fumonisins (sum of $\mathrm{FB}_{1}$ and $\mathrm{FB}_{2}$ ), ochratoxin $A$ (OTA) and zearalenone (ZEA or ZEN) while a recommended level is defined for the sum of T-2 and HT-2 toxin. ${ }^{43}$ The structures of six mycotoxins used in this thesis work are depicted in Figure 11. 
<smiles>COc1cc2c(c3c1CCC3=O)[C@H]1C=CO[C@@H]1OC2=O</smiles>

Aflatoxin $B_{1}\left(\mathrm{AFB}_{1}\right)$<smiles>C[C@H]1Cc2c(Cl)cc(C(=O)N[C@@H](Cc3ccccc3)C(=O)O)c(O)c2C(=O)O1</smiles>

Ochratoxin A (OTA)

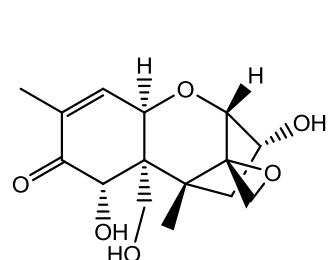

Deoxynivalenol (DON)

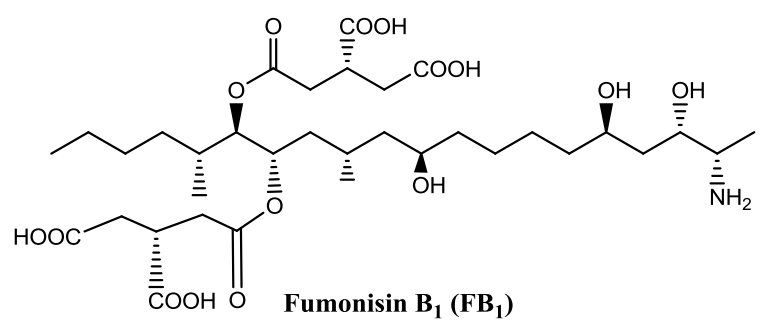<smiles>CO[C@H](C)CCCC(=O)CCC/C=C/c1cc(O)cc(O)c1C(=O)O</smiles>

Zearalenone (ZEA or ZEN)

Figure 11. Chemical structure of six relevant mycotoxins.

There are several techniques for detection of mycotoxins with their own pros and cons. ${ }^{44-46}$ Most conventional methods for quantitative analysis of multiple mycotoxins are based upon liquid chromatography (LC) or gas chromatography (GC) in combination with mass spectrometry (MS). ${ }^{47}$ Despite their high sensitivity, these techniques require extensive sample preparation, expert personnel for operation, are expensive and are not suitable for in-field applications. Recent MS techniques using ambient ionization have partially overcome the limitations by reducing the cost and sample preparation. ${ }^{48}$ In view of the cost and in-field use, immunological assays have gained substantial popularity. ${ }^{49}$ Enzyme-linked immunosorbent assays (ELISA) have dominated the field of immunoassays for several decades along with lateral flow immunoassay (LFI). ${ }^{50}$ Other techniques have been continuously emerging such as metal-enhanced fluorescence, ${ }^{51}$ chemiluminescent immunoassay, ${ }^{52,53}$ immuno-polymerase chain reaction, ${ }^{54}$ real-time electrochemical profiling, ${ }^{55}$ fluorescent immunosorbent assay ${ }^{56}$ and microsphere-based assays. ${ }^{57,58}$ Besides these techniques SPR-based biosensors have found their own niche and have emerged as a reliable, label-free and sensitive alternative immunochemical method for the semi-quantitative detection of mycotoxins. ${ }^{59-62}$ The development of an SPR biosensor for detection of multiple mycotoxins involves several steps as explained in the following sections. 
Table 1. Various techniques for characterization of a surface before and after its modification.

\begin{tabular}{|c|c|c|c|}
\hline Technique & $\begin{array}{l}\text { Information } \\
\text { obtained }\end{array}$ & $\begin{array}{l}\text { Spatial } \\
\text { Resolution }\end{array}$ & Pros \& Cons \\
\hline $\mathrm{AFM}^{63}$ & $\begin{array}{l}\text { Surface } \\
\text { morphology }\end{array}$ & $1-20 \mathrm{~nm}$ & $\begin{array}{l}\text { + 3D surface profile } \\
\text { - Scan speed slow compared to } \\
\text { SEM }\end{array}$ \\
\hline SEM $^{64}$ & $\begin{array}{l}\text { Surface } \\
\text { morphology }\end{array}$ & $8 \mathrm{~nm}$ & $\begin{array}{l}\text { + Fast scanning } \\
\text { - No 3D surface profile }\end{array}$ \\
\hline Auger ${ }^{65}$ & $\begin{array}{l}\text { Elemental } \\
\text { composition }\end{array}$ & $10-20 \mathrm{~nm}$ & $\begin{array}{l}\text { + High spatial resolution } \\
\text { - Quantification of data is } \\
\text { complex }\end{array}$ \\
\hline Contact angle ${ }^{66}$ & $\begin{array}{l}\text { Surface } \\
\text { hydrophobicity }\end{array}$ & $5 \mathrm{~mm}$ & $\begin{array}{l}\text { + Quick information } \\
\text { - No information on chemical } \\
\text { composition }\end{array}$ \\
\hline $\mathrm{XPS}^{65}$ & $\begin{array}{l}\text { Elements and } \\
\text { their oxidation } \\
\text { states, bonds, } \\
\text { thickness }\end{array}$ & $100 \mu \mathrm{m}$ & $\begin{array}{l}\text { + Chemical specificity } \\
\text { - Lower spatial resolution } \\
\text { compared to Auger }\end{array}$ \\
\hline $\begin{array}{l}\text { DART- } \\
\text { HRMS }^{67,68}\end{array}$ & $\begin{array}{l}\text { Surface bound } \\
\text { species }\end{array}$ & $2-3 \mathrm{~mm}$ & $\begin{array}{l}\text { + No solvent or high vacuum, } \\
\text { detailed molecular information } \\
\text { - Some chemical groups cannot } \\
\text { be analyzed }\end{array}$ \\
\hline
\end{tabular}

\section{Surface chemistry}

Surface chemistry plays an important role in the development of an SPR assay. The chosen chemistry, in addition to allowing covalent attachment of ligands, also prevents non-specific adsorption (fouling) of other components of the sample. ${ }^{69,70}$ Due to the lack of intrinsic selectivity of SPR, any binding on the surface including fouling gives a signal thus leading to false results. A widespread approach to reduce non-specific adsorption is to modify the surface with hydrophilic polymers. Carboxymethylated dextran (CMD) coatings are the most popular antifouling layers used in commercial SPR sensors. ${ }^{71}$ Selfassembled monolayers (SAMs) composed of oligo(ethylene glycol) (OEG) ${ }^{72}$ and poly(ethylene glycol) (PEG) tethered alkanethiol chains terminated with different functional groups have been used as alternatives to CMD. ${ }^{73}$ PEGs are more stable compared to OEGs and display improved antifouling characteristics, and are used more often. ${ }^{69}$ PEGs of fixed lengths can either be directly attached to the surface using grafted to approach, or variable lengths can be obtained using PEG methacrylate (PEGMA) in a 
grafting-from approach. In recent years, zwitterionic polymers of carboxybetaine methacrylate (CBMA) and sulfobetaine methacrylate (SBMA) have been developed as a promising approach towards antifouling coating. ${ }^{74}$ Due to their methacrylate functional group, surface-bound polymers of these zwitterions can be obtained using surfaceinitiated atom transfer radical polymerization (SI-ATRP). A variety of PEG and zwitterionic monomers is available, in many cases commercially, and an example of each is shown in Figure 12. In addition to the modification of the biosensor, characterization of the biosensor chip before and after modification is important and information about this is often missing in biosensing literature. ${ }^{75}$ Several methods used in this thesis for the detailed characterization of the sensor chip (Table 1) will be shown to provide highly useful complementary information.

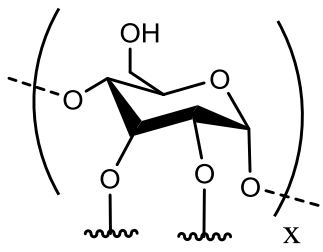

CMD<smiles>C=C(C)C(=O)NCCC[N+](C)(C)CCCC(=O)[O-]</smiles>

CBMA<smiles>C=C(C)C(=O)OCC[N+](C)(C)CCC[SeH]</smiles>

SBMA<smiles>C=C(C)C(=O)OCCCCCCC(C)OCC(C)(C)OCCCN</smiles>

PEGMA

Figure 12. Chemical structures of the commonly used form of different antifouling chemistries: carboxymethylated dextran (CMD), carboxybetaine methacrylate monomer (CBMA), sulfobetaine methacrylate monomer (SBMA), polyethylene glycol (PEG) and PEG methacrylate monomer (PEGMA).

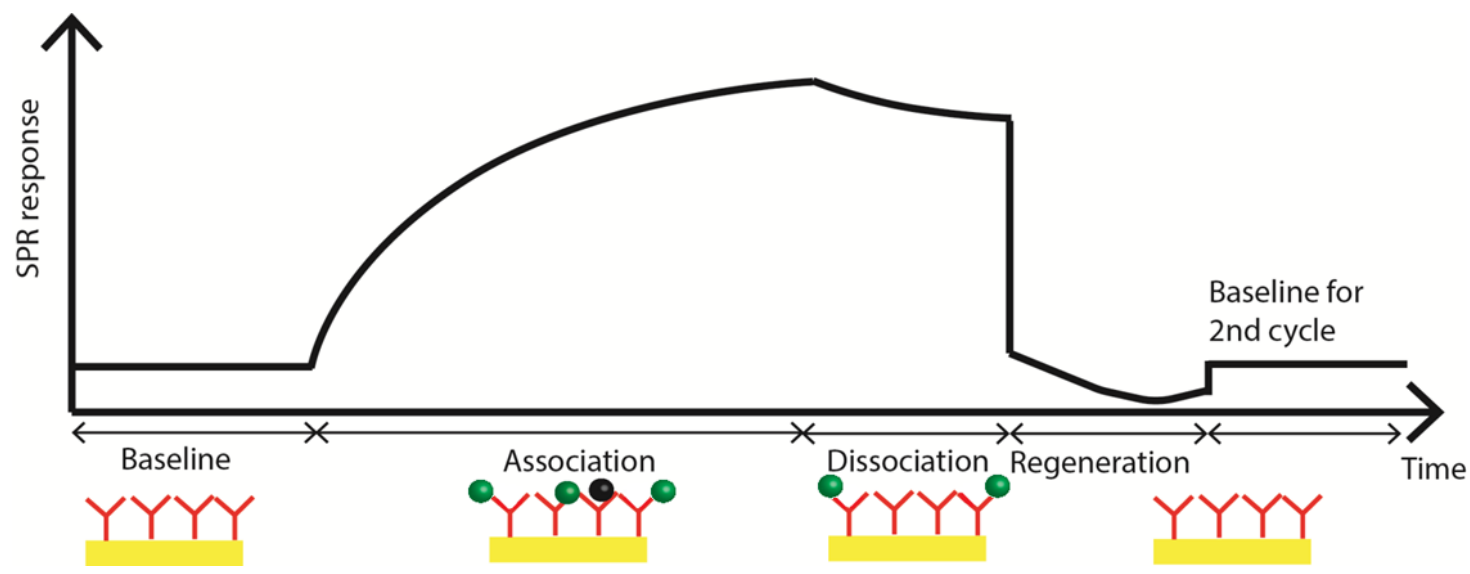

Figure 13. A typical SPR cycle consisting of baseline, association phase, dissociation phase, regeneration followed by baseline for the second cycle. A model system (direct assay) with antibodies immobilized on the surface is shown with a sample consisting of a specific analyte (green balls) and a non-specific component from the sample (black balls). 


\section{SPR immunoassay development}

A typical SPR immunoassay development starts with immobilization of a ligand on the SPR chip and the choice of ligand depends on the type of SPR assay and the analyte of interest. In a direct assay (Figure 13), antibodies against the analyte of interest are immobilized on the surface. The SPR response upon injection of a solution containing different concentrations of analyte is recorded. As the SPR response is directly proportional to the mass of binding molecule, the response increases with increasing concentration of the analyte. However, for small analytes ( $M W<1 \mathrm{kDa})$, the signal generated by the analyte itself is typically not sufficient. Therefore, a signal enhancer with a high molecular weight, such as a protein, is required. Furthermore, antibodies directly attached to the surface are susceptible to denaturation. Thus, indirect assays are preferred for small-molecule detection, such as for mycotoxins. In an indirect assay (Figure 14), either the analyte itself or a protein conjugate of the analyte is immobilized on the surface. The SPR response upon injection of a fixed concentration of antibodies mixed with different concentrations of analyte is recorded. Since the free analytes in the solution compete for binding with the antibodies, the SPR response decreases with increasing concentration of analyte in the sample (inhibition). These assays are also referred to as competitive inhibition assays. Protein conjugates of analytes are used as ligands in competitive inhibition assays as they offer a more generic approach for immobilization of the available conjugates. Direct immobilization of small molecules provides more stable surfaces compared to protein conjugates as the latter are more sensitive to regeneration. However, direct immobilization can be challenging as only one or a limited number of functional groups can be used for immobilization without blocking the antibody binding sites. Depending on the surface chemistry and the ligand to be immobilized there are several approaches for immobilization of ligands on the surface, including but not limited to amine coupling, thiol coupling, thiol-disulfide exchange, maleimide coupling. ${ }^{76,77}$ For protein conjugates and antibodies, the most used method is amine coupling of lysine residues of the ligand with activated carboxylic acid on the surface (resulting from specific surface chemistry). ${ }^{78}$ However, the direct immobilization of mycotoxins is governed by the functional groups present in the toxins themselves, as explained in further detail in Chapter 4. In Biacore instruments, depending on the reagents used, the immobilization of ligand can be monitored online or performed offline using the surface prep unit. In contrast, in the iSPR instruments (IBIS and imaging nanoplasmonics), the ligand is either immobilized using a microspotter or manually. In all cases, the SPR response of the chip when buffer is flushed over a chip with immobilized ligand serves as the baseline (Figure 13). 


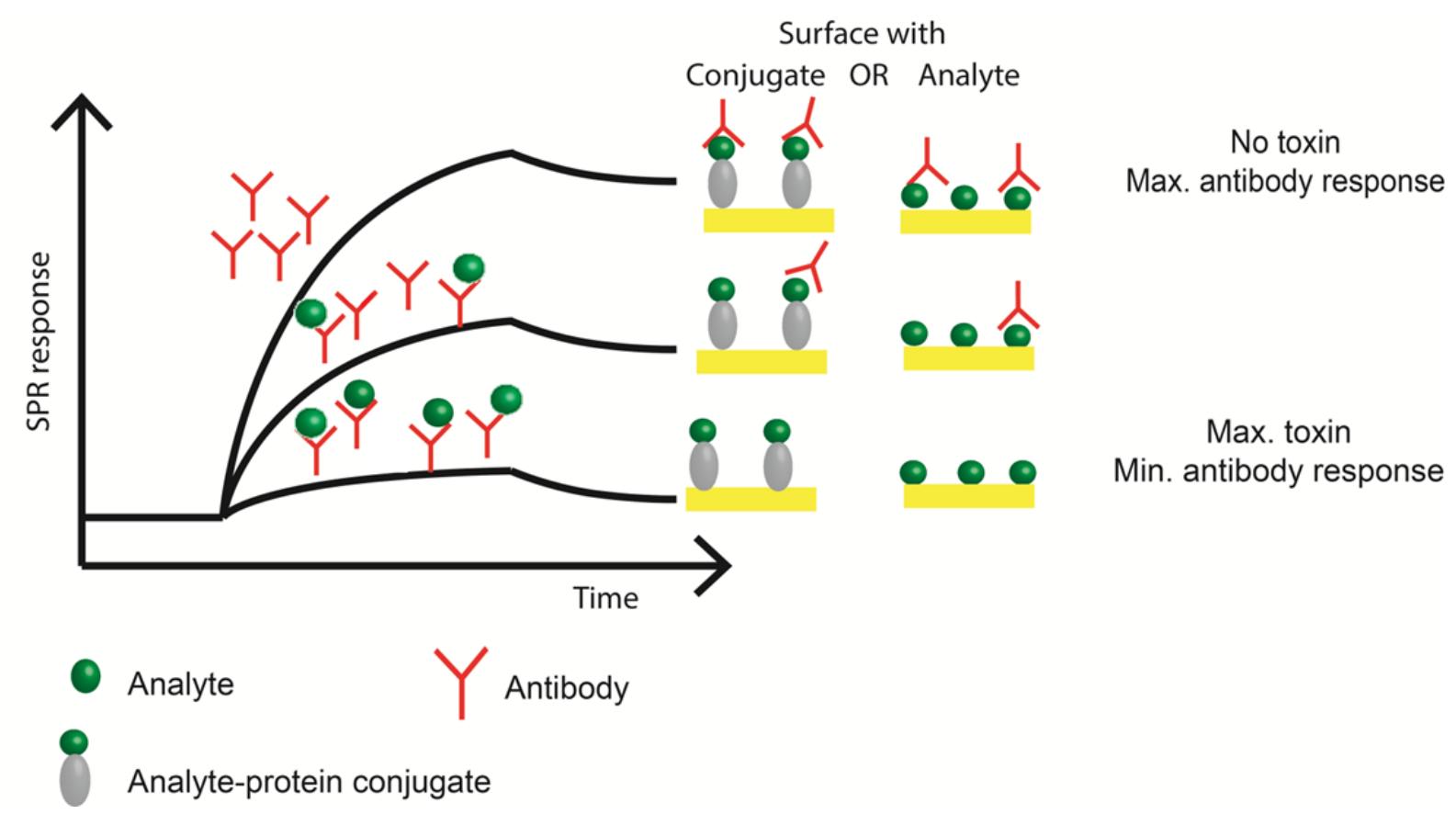

Figure 14. Schematic representation of an indirect SPR assay. Either the analyte itself or a protein conjugate of the analyte is immobilized on the surface. The response of an antibody in the presence or absence of toxin is recorded.

In a typical SPR sensorgram, as the sample is injected, a change in SPR signal occurs (association, Figure 13). The sample is prepared in the same buffer as the running buffer for SPR to minimize bulk changes in refractive index. The initial response is a combination of any minor change in bulk refractive index and binding. After the injection has finished and the flow of buffer restarts, the response due to bulk refractive index changes, adhesion of non-specifically binding molecules or loosely bound specific molecules is removed (dissociation, Figure 13). The response in the stable part of the dissociation phase relative to the starting baseline is used as the SPR response. The SPR response increases (in direct assay) or decreases (in an indirect competitive inhibition assay) with an increasing concentration of the analyte. Therefore, in a competitive inhibition assay, the highest response is obtained upon injection of only antibody and needs to be optimized in such a way that the lowest signal (highest concentration of analyte) is still above the noise of the instrument. Several factors such as concentration of antibodies, buffer composition and $\mathrm{pH}$ play a vital role. ${ }^{79}$

The possibility of re-use of the sensor chips is one of the advantages of SPR over other immunoassays. Therefore, regeneration is performed at the end of each cycle (Figure 13) to remove the bound molecules and to prepare the chip for the next cycle using the ligands on the surface. However, regeneration can be critical, especially in the case of multiplex assay development, due to the varying sensitivities of different ligands towards the regeneration buffer. A variety of regeneration buffers can be used ${ }^{80}$ and 
need to be optimized based on the application. Surfaces with antibodies or protein conjugates are more sensitive to harsh regeneration solutions. On the other hand, surfaces with covalently bound analytes are more stable and can be re-used many more times.

\section{Signal enhancement}

A low signal/noise ratio in the SPR signal is a serious problem in the detection of small molecules or of large molecules in low concentrations. Use of nanostructured sensor chips has been proposed for sensitivity enhancement due to the possibility of coupling of normal SPR and localized SPR signals. ${ }^{81-84}$ However, accurate positioning of the ligand is required at the region where the maximum of the evanescent wave from the LSPR lies. This effect known as confinement enhancement ${ }^{85,86}$ of SPR signals would require very strict control of the fabrication process and still remains a challenge.

Changes in instrumental design do not lead to sufficient signal enhancement, and therefore experimental optimization is also required. ${ }^{87}$ Nanoparticle-enhanced SPR studies, one of the most widely used approaches, are mostly based on the use of free or functionalized gold nanoparticles. ${ }^{88,89}$ The enhancement is attributed to the higher molecular weight of gold NPs conjugates compared to conjugates that are not bound to NPs and to the electronic coupling interaction between the gold NPs and the surface plasmon wave associated with the SPR gold film. ${ }^{12}$ Other techniques that have been used for signal enhancement are based on enzymatic amplification or polymerization approaches. ${ }^{90}$

\section{Effect of cross-talk and cross-reactivity}

Cross-talk, the binding of one mycotoxin to an antibody against another mycotoxin, is a major challenge of multiplex mycotoxin analysis as it can lead to false positives. Use of monoclonal antibodies has helped to overcome this problem to a great extent, however, careful screening is required. During the assay development, the individual antibodies are tested followed by the mixture to account for any cross-reactivity. From this point of view, imaging SPR has an added advantage as all the mycotoxins or their conjugates can be immobilized on one chip and any cross-talk can be easily visualized. In addition to the various parent mycotoxins, conjugated forms of mycotoxins, formed by the detoxification metabolic processes of plants, can also be present in the sample. ${ }^{91}$ Due to their structural similarity, some conjugates can cross-react with the antibodies and this can lead to an overestimation of the amount of target mycotoxin present in the sample. Cross-reactivity is calculated by comparison of the calibration curve of the conjugates with that of the parent toxin. However, cross-reactivity can also be favorable when regulations are set for the sum of the different conjugates or toxins rather than for only the toxin itself (e.g. T-2 
and $\mathrm{HT}-2, \mathrm{FB}_{1}$ and $\mathrm{FB}_{2}$, and $\mathrm{AFB}_{2}, \mathrm{AFG}_{1}$ and $\mathrm{AFG}_{2}$ ). Furthermore, in a competitive screening assay, cross-reactivity would result in a positive result, thus qualifying the sample for quantitative confirmatory analysis and even helping to identify the crossreacting analyte.

\section{Coupling of SPR with ambient ionization mass spectrometry for identification of cross-reacting analytes}

SPR is a powerful tool for detection of a wide range of analytes, but does not give structural information about the binding analyte. Furthermore, cross-reactivity of the antibodies towards other analytes or conjugates of the same analyte cannot be distinguished by SPR, and can lead to overestimation of the main analyte. Therefore, coupling of SPR chips using mass spectrometry allows not only identification of the main analyte of interest, but might also help find cross-reacting analytes. ${ }^{92}$ In the past, offline methods have been used for analysis of the analytes after SPR experiments, but this can lead to loss of sample. ${ }^{93}$ Online coupling methods developed involve complicated and highly specialized setups. ${ }^{94-96}$ One of the most promising approaches with matrix-assisted laser desorption ionization (MALDI), is not suitable for analysis of small molecules $(<0.6$ $\mathrm{kDa}$ ) as the matrix required for the method interferes with the analysis. ${ }^{97,98}$ Therefore, there is a demand for a simplified coupling of SPR with mass spectrometry.

Ambient ionization methods for mass spectrometry, such as direct analysis in real time (DART) ${ }^{67}$ and desorption electrospray ionization (DESI), ${ }^{99,100}$ have gained significant interest. The benefits of these techniques is that analyses can be performed at room temperature, under atmospheric conditions, often require minimal sample preparation and are suitable for small molecules. ${ }^{68}$ Direct spray, ${ }^{101}$ one of the ambient ionization methods, allows the analysis of a sample loaded onto a solid substrate (paper, metal, wood etc. $)^{102-104}$ where the ions of the analyte generated using an organic solvent in combination with a high voltage (HV) are analyzed. Despite their simplicity, all these methods rely on desorbing analytes, either already present or spiked on the substrate and the selectivity is dependent on the mass spectrometer. Direct analysis of a SPR biochip for complex samples containing small analytes yields several challenges, including the interference of other components of the sample as well as ion suppressants (buffer components). Recently, in an attempt to get rid of these possible interferences, SPME combined with desorption electrospray ionization (DESI) source was used. ${ }^{105}$ The method offers sample enrichment by selectively capturing the analyte from the sample mixture. A washing step after enrichment and before the MS analysis helps to get rid of the buffer components. An SPR chip with antibodies can provide the required enrichment of analytes, and the buffer components can be easily washed off during the dissociation 
phase (Figure 13). Therefore, combination of SPR with direct spray would offer a simple coupling of SPR and MS for analysis of small molecules.

\section{Aim of the research}

SPR-based biosensors have emerged as a fast, label-free and sensitive immunochemical method for the semi-quantitative detection of a range of analytes including mycotoxins. The real-time monitoring of the interaction kinetics and reusability of the biosensor chips offers additional advantages. The development of imaging SPR instruments added the possibility of multiplexing thus expanding the horizon of SPR instruments even further. An instrument with all these features and in a portable format was only recently developed, thanks to a nanostructured biosensor chip, in a prototype format by Plasmore Srl. (Italy). Furthermore, very few prototype SPR instruments have been thoroughly studied, validated, benchmarked against conventional instruments or commercialized. ${ }^{33}$ Therefore, the aim of this research was to test and further develop a prototype nanostructured iSPR biosensor, with a focus on surface modification and detailed characterization of the biosensor chip, and on in-field and at-line applicability in the food industry. Furthermore, a simplified coupling of SPR and MS was developed that allows identification of the mycotoxins of interest along with any other cross-reacting analytes.

\section{Outline of the thesis}

The aim of the research was achieved in several sub-projects as outlined below. The surface modification, in-depth surface characterization and the antifouling performance of the nanostructured iSPR chip is described in Chapter 2. Results were compared with conventional flat gold chips having the same surface chemistries. Proof-of-principle biosensing was demonstrated using biotin-streptavidin as a model system.

In Chapter 3 a 6-plex competitive inhibition assay for mycotoxins in barley was developed using the nanostructured iSPR instrument. As a benchmark a double 3-plex SPR assay (using Biacore) was developed. Preliminary in-house validation and measurement of naturally contaminated barley were performed.

The iSPR assay developed in Chapter $\mathbf{3}$ was further optimized and a complete assay for detection of two mycotoxins in beer was developed using the nanostructured iSPR instrument and is described in Chapter 4.

A simplified coupling of surface plasmon resonance to mass spectrometry is described in Chapter 5. The SPR chips containing antibodies against deoxynivalenol (DON) were analyzed, after injection of sample in the SPR, by ambient mass 
spectrometry that allowed confirmation of the presence of DON as well as identification of cross-reacting conjugates.

Chapter 6 contains a general discussion about the research topic described in this thesis and outlines possible future developments for iSPR instruments. 


\section{References}

1. Abdulhalim, I.; Zourob, M.; Lakhtakia, A. Surface plasmon resonance for biosensing: A minireview. Electromagnetics 2008, 28, 214-242.

2. Guo, X. Surface plasmon resonance based biosensor technique: A review. J. Biophotonics 2012, 5, 483-501.

3. Singh, P. SPR biosensors: Historical perspectives and current challenges. Sens. Actuat. B: Chem. 2016, 229, 110-130.

4. Schasfoort, R.; Tudos, A., Handbook of surface plasmon resonance. The Royal Society of Chemistry: Cambridge, 2008.

5. Yeatman, E.; Ash, E. A. Surface plasmon microscopy. Electronics Lett. 1987, 23, 10911092.

6. $\quad$ Rothenhaüsler, B.; Knoll, W. Surface-plasmon microscopy. Nature 1988, 332, 615-617.

7. Campbell, C. T.; Kim, G. SPR microscopy and its applications to high-throughput analyses of biomolecular binding events and their kinetics. Biomaterials 2007, 28, 2380-2392.

8. Willets, K. A.; Van Duyne, R. P. Localized surface plasmon resonance spectroscopy and sensing. Annu. Rev. Phys. Chem. 2007, 58, 267-297.

9. Xiang, G.; Zhang, N.; Zhou, X. Localized surface plasmon resonance biosensing with large area of gold nanoholes fabricated by nanosphere lithography. Nanoscale Res. Lett. 2010, 5, 818-822.

10. Petryayeva, E.; Krull, U. J. Localized surface plasmon resonance: Nanostructures, bioassays and biosensing-A review. Anal. Chim. Acta 2011, 706, 8-24.

11. Joshi, G. K.; McClory, P. J.; Dolai, S.; Sardar, R. Improved localized surface plasmon resonance biosensing sensitivity based on chemically-synthesized gold nanoprisms as plasmonic transducers. J Mater. Chem. 2012, 22, 923-931.

12. Bolduc, O. R.; Masson, J.-F. Advances in surface plasmon resonance sensing with nanoparticles and thin films: Nanomaterials, surface chemistry, and hybrid plasmonic techniques. Anal. Chem. 2011, 83, 8057-8062.

13. Cheng, K.; Wang, S. J.; Cui, Z. G.; Li, Q. Q.; Dai, S. X.; Du, Z. L. Large-scale fabrication of plasmonic gold nanohole arrays for refractive index sensing at visible region. Appl. Phys. Lett. 2012, 100, 253101-253104.

14. Im, H.; Sutherland, J. N.; Maynard, J. A.; Oh, S.-H. Nanohole-based surface plasmon resonance instruments with improved spectral resolution quantify a broad range of antibodyligand binding kinetics. Anal. Chem. 2012, 84, 1941-1947.

15. Najiminaini, M.; Ertorer, E.; Kaminska, B.; Mittler, S.; Carson, J. J. L. Surface plasmon resonance sensing properties of a 3D nanostructure consisting of aligned nanohole and nanocone arrays. Analyst 2014, 139, 1876-1882.

16. Valsesia, A.; Colpo, P.; Rossi, F.; Marabelli, F. A surface plasmon resonance sensing method and sensing system. European Patent 2010, EP 2264438A1.

17. Giudicatti, S.; Marabelli, F.; Valsesia, A.; Pellacani, P.; Colpo, P.; Rossi, F. Interaction among plasmonic resonances in a gold film embedding a two-dimensional array of polymeric nanopillars. J. Opt. Soc. Am. B 2012, 29, 1641-1647.

18. Parsons, J.; Hendry, E.; Burrows, C. P.; Auguié, B.; Sambles, J. R.; Barnes, W. L. Localized surface-plasmon resonances in periodic nondiffracting metallic nanoparticle and nanohole arrays. Phys. Rev. B 2009, 79, 0734121-0734124.

19. Valsesia, A.; Marabelli, F.; Giudicatti, S.; Marchesini, G. R.; Rossi, F.; Colpo, P. SPR sensor device with nanostructure. World Patent 2013, WO 2013/007448 A1.

20. Jönsson, U.; Fägerstam, L.; Ivarsson, B.; Johnsson, B.; Karlsson, R.; Lundh, K.; Löfas, S.; Persson, B.; Roos, H.; Rönnberg, I.; Sjölander, S.; Stenberg, E.; Stahlberg, R.; Urbaniczky, C.; Ostlin, H.; Malmqvist, M. Real-time biospecific interaction analysis using surface plasmon resonance and a sensor chip technology. BioTechniques 1991, 11, 620-627.

21. SPR pages, SPR instruments. http://www.sprpages.nl/instruments (6 May 2016).

22. Beusink, J. B.; Lokate, A. M. C.; Besselink, G. A. J.; Pruijn, G. J. M.; Schasfoort, R. B. M. Angle-scanning SPR imaging for detection of biomolecular interactions on microarrays. Biosens. Bioelectron. 2008, 23, 839-844.

23. Raz, S. R. On-chip food safety monitoring: Multi-analyte screening with imaging surface plasmon resonance-based biosensor. PhD thesis, Wageningen University, Wageningen, the Netherlands, 2010.

24. van Beers, J. J. B. C.; Raijmakers, R.; Alexander, L.-E.; Stammen-Vogelzangs, J.; Lokate, A. M. C.; Heck, A. J. R.; Schasfoort, R. B. M.; Pruijn, G. J. M. Mapping of citrullinated fibrinogen $\mathrm{B}$-cell epitopes in rheumatoid arthritis by imaging surface plasmon resonance. Arthrit. Res. Ther. 2010, 12, R219-R219. 
25. Abdiche, Y. N.; Miles, A.; Eckman, J.; Foletti, D.; Van Blarcom, T. J.; Yeung, Y. A.; Pons, J.; Rajpal, A. High-throughput epitope binning assays on label-free array-based biosensors can yield exquisite epitope discrimination that facilitates the selection of monoclonal antibodies with functional activity. PLOS ONE 2014, 9, e924511-e9245116.

26. Mauriz, E.; Calle, A.; Montoya, A.; Lechuga, L. M. Determination of environmental organic pollutants with a portable optical immunosensor. Talanta 2006, 69, 359-364.

27. Chinowsky, T. M.; Soelberg, S. D.; Baker, P.; Swanson, N. R.; Kauffman, P.; Mactutis, A.; Grow, M. S.; Atmar, R.; Yee, S. S.; Furlong, C. E. Portable 24-analyte surface plasmon resonance instruments for rapid, versatile biodetection. Biosens. Bioelectron. 2007, 22, 2268-2275.

28. Kim, S. J.; Gobi, K. V.; Iwasaka, H.; Tanaka, H.; Miura, N. Novel miniature SPR immunosensor equipped with all-in-one multi-microchannel sensor chip for detecting lowmolecular-weight analytes. Biosens. Bioelectron. 2007, 23, 701-707.

29. Marchesini, G. R.; Koopal, K.; Meulenberg, E.; Haasnoot, W.; Irth, H. Spreeta-based biosensor assays for endocrine disruptors. Biosens. Bioelectron. 2007, 22, 1908-1915.

30. Fernández, F.; Hegnerová, K.; Piliarik, M.; Sanchez-Baeza, F.; Homola, J.; Marco, M. P. A label-free and portable multichannel surface plasmon resonance immunosensor for on site analysis of antibiotics in milk samples. Biosens. Bioelectron. 2010, 26, 1231-1238.

31. Preechaburana, P.; Gonzalez, M. C.; Suska, A.; Filippini, D. Surface plasmon resonance chemical sensing on cell phones. Angew. Chem. Int. Ed. 2012, 51, 11585-11588.

32. Liu, Y.; Liu, Q.; Chen, S.; Cheng, F.; Wang, H.; Peng, W. Surface plasmon resonance biosensor based on smart phone platforms. Sci. Rep. 2015, 5, 128641-128649.

33. Dahlin, A. B. Sensing applications based on plasmonic nanopores: The hole story. Analyst 2015, 140, 4748-4759.

34. Love, J. C.; Estroff, L. A.; Kriebel, J. K.; Nuzzo, R. G.; Whitesides, G. M. Self-assembled monolayers of thiolates on metals as a form of nanotechnology. Chem. Rev. 2005, 105, 1103-1170.

35. Cheng, Z.; Wang, Z.; Gillespie, D. E.; Lausted, C.; Zheng, Z.; Yang, M.; Zhu, J. Plain silver surface plasmon resonance for microarray application. Anal. Chem. 2015, 87, 1466-1469.

36. Ruffato, G.; Romanato, F. Grating-coupled surface plasmon resonance in conical mounting with polarization modulation. Opt. Lett. 2012, 37, 2718-2720.

37. Petefish, J. W.; Hillier, A. C. Multipitched diffraction gratings for surface plasmon resonanceenhanced infrared reflection absorption spectroscopy. Anal. Chem. 2015, 87, 10862-10870.

38. Giudicatti, S.; Valsesia, A.; Marabelli, F.; Colpo, P.; Rossi, F. Plasmonic resonances in nanostructured gold/polymer surfaces by colloidal lithography. Phys. Status Solidi A 2010, 207, 935-942.

39. Sharma, A. K.; Jha, R.; Gupta, B. D. Fiber-optic sensors based on surface plasmon resonance: A comprehensive review. IEEE Sens. J. 2007, 7, 1118-1129.

40. DeVries, J. W.; Trucksess, M. W.; Jackson, L. S., Mycotoxins and food safety. Springer: New York, 2002.

41. Schatzmayr, G.; Streit, E. Global occurrence of mycotoxins in the food and feed chain: Facts and figures. World Mycotoxin J. 2013, 6, 213-222.

42. Commission regulation (EC) No 1881/2006 of 19 December 2006 setting maximum levels for certain contaminants in foodstuffs. Off. J. Eur. Union 2006, L364, 5-24.

43. Commission recommendation of 27 March 2013 on the presence of T-2 and HT-2 toxin in cereals and cereal products. Off. J. Eur. Union 2013, L91, 12-15.

44. Berthiller, F.; Brera, C.; Crews, C.; Iha, M. H.; Krska, R.; Lattanzio, V. M. T.; MacDonald, S.; Malone, R. J.; Maragos, C.; Solfrizzo, M.; Stroka, J.; Whitaker, T. B. Developments in mycotoxin analysis: An update for 2013-2014. World Mycotoxin J. 2015, 8, 5-35.

45. Berthiller, F.; Brera, C.; Crews, C.; Iha, M. H.; Krska, R.; Lattanzio, V. M. T.; MacDonald, S.; Malone, R. J.; Maragos, C.; Solfrizzo, M.; Stroka, J.; Whitaker, T. B. Developments in mycotoxin analysis: An update for 2014-2015. World Mycotoxin J. 2016, 9, 5-30.

46. Chauhan, R.; Singh, J.; Sachdev, T.; Basu, T.; Malhotra, B. D. Recent advances in mycotoxins detection. Biosens. Bioelectron. 2016, 81, 532-545.

47. Berthiller, F.; Sulyok, M.; Krska, R.; Schuhmacher, R. Chromatographic methods for the simultaneous determination of mycotoxins and their conjugates in cereals. Int. J. Food Microbiol. 2007, 119, 33-37.

48. Vaclavik, L.; Zachariasova, M.; Hrbek, V.; Hajslova, J. Analysis of multiple mycotoxins in cereals under ambient conditions using direct analysis in real time (DART) ionization coupled to high resolution mass spectrometry. Talanta 2010, 82, 1950-1957.

49. Goryacheva, I. Y.; De Saeger, S., Immunochemical methods for rapid mycotoxin detection in food and feed. In: Determining mycotoxins and mycotoxigenic fungi in food and feed, De Saeger, S., Ed. Woodhead Publishing: Cambridge, 2011; pp 135-167. 
50. Li, W.; Powers, S.; Dai, S. Y. Using commercial immunoassay kits for mycotoxins: 'Joys and sorrows'? World Mycotoxin J. 2014, 7, 417-430.

51. Todescato, F.; Antognoli, A.; Meneghello, A.; Cretaio, E.; Signorini, R.; Bozio, R. Sensitive detection of ochratoxin $A$ in food and drinks using metal-enhanced fluorescence. Biosens. Bioelectron. 2014, 57, 125-132.

52. Yang, L.; Zhang, Y.; Li, R.; Lin, C.; Guo, L.; Qiu, B.; Lin, Z.; Chen, G. Electrochemiluminescence biosensor for ultrasensitive determination of ochratoxin $A$ in corn samples based on aptamer and hyperbranched rolling circle amplification. Biosens. Bioelectron. 2015, 70, 268-274.

53. Zangheri, M.; Di Nardo, F.; Anfossi, L.; Giovannoli, C.; Baggiani, C.; Roda, A.; Mirasoli, M. A multiplex chemiluminescent biosensor for type B-fumonisins and aflatoxin B1 quantitative detection in maize flour. Analyst 2015, 140, 358-365.

54. Liu, X.; Xu, Y.; Xiong, Y.-h.; Tu, Z.; Li, Y.-p.; He, Z.-y.; Qiu, Y.-I.; Fu, J.-h.; Gee, S. J.; Hammock, B. D. VHH phage-based competitive real-time immuno-polymerase chain reaction for ultrasensitive detection of ochratoxin A in cereal. Anal. Chem. 2014, 86, 7471-7477.

55. Olcer, Z.; Esen, E.; Muhammad, T.; Ersoy, A.; Budak, S.; Uludag, Y. Fast and sensitive detection of mycotoxins in wheat using microfluidics based real-time electrochemical profiling. Biosens. Bioelectron. 2014, 62, 163-169.

56. Beloglazova, N. V.; Speranskaya, E. S.; Wu, A.; Wang, Z.; Sanders, M.; Goftman, V. V.; Zhang, D.; Goryacheva, I. Y.; De Saeger, S. Novel multiplex fluorescent immunoassays based on quantum dot nanolabels for mycotoxins determination. Biosens. Bioelectron. 2014, 62, 59-65.

57. Peters, J.; Bienenmann-Ploum, M.; de Rijk, T.; Haasnoot, W. Development of a multiplex flow cytometric microsphere immunoassay for mycotoxins and evaluation of its application in feed. Mycotoxin Res. 2011, 27, 63-72.

58. Peters, J.; Cardall, A.; Haasnoot, W.; Nielen, M. W. F. 6-plex microsphere immunoassay with imaging planar array detection for mycotoxins in barley. Analyst 2014, 139, 3968-3976.

59. Homola, J. Surface plasmon resonance sensors for detection of chemical and biological species. Chem. Rev. 2008, 108, 462-493.

60. Li, Y.; Liu, X.; Lin, Z. Recent developments and applications of surface plasmon resonance biosensors for the detection of mycotoxins in foodstuffs. Food Chem. 2012, 132, 15491554.

61. Meneely, J. P.; Elliott, C. T. Rapid surface plasmon resonance immunoassays for the determination of mycotoxins in cereals and cereal-based food products. World Mycotoxin J. 2014, 7, 491-505.

62. Karczmarczyk, A.; Dubiak-Szepietowska, M.; Vorobii, M.; Rodriguez-Emmenegger, C.; Dostálek, J.; Feller, K.-H. Sensitive and rapid detection of aflatoxin $M 1$ in milk utilizing enhanced SPR and p(HEMA) brushes. Biosens. Bioelectron. 2016, 81, 159-165.

63. Gan, Y. Atomic and subnanometer resolution in ambient conditions by atomic force microscopy. Surf. Sci. Rep. 2009, 64, 99-121.

64. Kaminskyj, S. G. W.; Dahms, T. E. S. High spatial resolution surface imaging and analysis of fungal cells using SEM and AFM. Micron 2008, 39, 349-361.

65. Watts, J. F.; Wolstenholme, J., Comparison of XPS and AES with other analytical techniques. In: An introduction to surface analysis by XPS and AES, John Wiley \& Sons, Ltd: Chichester, 2005; pp 165-182.

66. Bell, M. S.; Shahraz, A.; Fichthorn, K. A.; Borhan, A. Effects of hierarchical surface roughness on droplet contact angle. Langmuir 2015, 31, 6752-6762.

67. Cody, R. B.; Laramée, J. A.; Durst, H. D. Versatile new ion source for the analysis of materials in open air under ambient conditions. Anal. Chem. 2005, 77, 2297-2302.

68. Manova, R. K.; Joshi, S.; Debrassi, A.; Bhairamadgi, N. S.; Roeven, E.; Gagnon, J.; Tahir, M. N.; Claassen, F. W.; Scheres, L. M. W.; Wennekes, T.; Schroën, K.; van Beek, T. A.; Zuilhof, H.; Nielen, M. W. F. Ambient surface analysis of organic monolayers using direct analysis in real time orbitrap mass spectrometry. Anal. Chem. 2014, 86, 2403-2411.

69. Mérian, T.; Goddard, J. M. Advances in nonfouling materials: Perspectives for the food industry. J. Agric. Food Chem. 2012, 60, 2943-2957.

70. Lowe, S.; O'Brien-Simpson, N. M.; Connal, L. A. Antibiofouling polymer interfaces: Poly(ethylene glycol) and other promising candidates. Polym. Chem. 2015, 6, 198-212.

71. Löfås, S.; Johnsson, B. A novel hydrogel matrix on gold surfaces in surface plasmon resonance sensors for fast and efficient covalent immobilization of ligands. J. Chem. Soc., Chem. Comm. 1990, 1526-1528.

72. Li, L.; Chen, S.; Zheng, J.; Ratner, B. D.; Jiang, S. Protein adsorption on oligo(ethylene glycol)-terminated alkanethiolate self-assembled monolayers: The molecular basis for nonfouling behavior. J. Phys. Chem. B 2005, 109, 2934-2941. 
73. Uchida, K.; Hoshino, Y.; Tamura, A.; Yoshimoto, K.; Kojima, S.; Yamashita, K.; Yamanaka, I.; Otsuka, H.; Kataoka, K.; Nagasaki, Y. Creation of a mixed poly(ethylene glycol) tetheredchain surface for preventing the nonspecific adsorption of proteins and peptides. Biointerphases 2007, 2, 126-130.

74. Wu, L.; Jasinski, J.; Krishnan, S. Carboxybetaine, sulfobetaine, and cationic block copolymer coatings: A comparison of the surface properties and antibiofouling behavior. J. Appl. Polym. Sci. 2012, 124, 2154-2170.

75. Duwez, A.-S. Exploiting electron spectroscopies to probe the structure and organization of self-assembled monolayers: A review. J. Electron Spectrosc. 2004, 134, 97-138.

76. Jonkheijm, P.; Weinrich, D.; Schröder, H.; Niemeyer, C. M.; Waldmann, H. Chemical strategies for generating protein biochips. Angew. Chem. Int. Ed. 2008, 47, 9618-9647.

77. Hermanson, G. T., Bioconjugate techniques. Academic Press: Boston, 2013.

78. O'Shannessy, D. J.; Brigham-Burke, M.; Peck, K. Immobilization chemistries suitable for use in the Biacore surface plasmon resonance detector. Anal. Biochem. 1992, 205, 132-136.

79. Skládal, P. Effect of methanol on the interaction of monoclonal antibody with free and immobilized atrazine studied using the resonant mirror-based biosensor. Biosens. Bioelectron. 1999, 14, 257-263.

80. Geuijen, K. P. M.; Schasfoort, R. B.; Wijffels, R. H.; Eppink, M. H. M. High-throughput and multiplexed regeneration buffer scouting for affinity-based interactions. Anal. Biochem. 2014, 454, 38-40.

81. Shalabney, A.; Abdulhalim, I. Sensitivity-enhancement methods for surface plasmon sensors. Laser Photonics Rev. 2011, 5, 571-606.

82. Breault-Turcot, J.; Masson, J.-F. Nanostructured substrates for portable and miniature SPR biosensors. Anal. Bioanal. Chem. 2012, 403, 1477-1484.

83. Kwon, M. J.; Lee, J.; Wark, A. W.; Lee, H. J. Nanoparticle-enhanced surface plasmon resonance detection of proteins at attomolar concentrations: Comparing different nanoparticle shapes and sizes. Anal. Chem. 2012, 84, 1702-1707.

84. Ertsgaard, C. T.; McKoskey, R. M.; Rich, I. S.; Lindquist, N. C. Dynamic placement of plasmonic hotspots for super-resolution surface-enhanced Raman scattering. ACS Nano 2014, 8, 10941-10946.

85. Krishnamoorthy, S.; Himmelhaus, M. Confinement-induced enhancement of antigenantibody interactions within binary nanopatterns to achieve higher efficiency of on-chip immunosensors. Adv. Mater. 2008, 20, 2782-2788.

86. Valsesia, A.; Colpo, P.; Mannelli, I.; Mornet, S.; Bretagnol, F.; Ceccone, G.; Rossi, F. Use of nanopatterned surfaces to enhance immunoreaction efficiency. Anal. Chem. 2008, 80, 1418-1424.

87. Goggins, S.; Frost, C. G. Approaches towards molecular amplification for sensing. Analyst 2016, 141, 3157-3218.

88. Hong, X.; Hall, E. A. H. Contribution of gold nanoparticles to the signal amplification in surface plasmon resonance. Analyst 2012, 137, 4712-4719.

89. Springer, T.; Ermini, M. L.; Spackova, B.; Jablonku, J.; Homola, J. Enhancing sensitivity of surface plasmon resonance biosensors by functionalized gold nanoparticles: Size matters. Anal. Chem. 2014, 86, 10350-10356.

90. Liu, Y.; Dong, Y.; Jauw, J.; Linman, M. J.; Cheng, Q. Highly sensitive detection of protein toxins by surface plasmon resonance with biotinylation-based inline atom transfer radical polymerization amplification. Anal. Chem. 2010, 82, 3679-3685.

91. Berthiller, F.; Crews, C.; Dall'Asta, C.; De Saeger, S.; Haesaert, G.; Karlovsky, P.; Oswald, I. P.; Seefelder, W.; Speijers, G.; Stroka, J. Masked mycotoxins: A review. Mol. Nutr. Food Res. 2013, 57, 165-186.

92. Stigter, E. C. A.; de Jong, G. J.; van Bennekom, W. P. Coupling surface-plasmon resonance and mass spectrometry to quantify and to identify ligands. Trends Anal. Chem. 2013, 45, 107-120.

93. Natsume, T.; Nakayama, H.; Jansson, Ö.; Isobe, T.; Takio, K.; Mikoshiba, K. Combination of biomolecular interaction analysis and mass spectrometric amino acid sequencing. Anal. Chem. 2000, 72, 4193-4198.

94. Marchesini, G. R.; Buijs, J.; Haasnoot, W.; Hooijerink, D.; Jansson, O.; Nielen, M. W. F. Nanoscale affinity chip interface for coupling inhibition SPR immunosensor screening with nano-LC TOF MS. Anal. Chem. 2008, 80, 1159-1168.

95. Zhang, Y.; Li, X.; Nie, H.; Yang, L.; Li, Z.; Bai, Y.; Niu, L.; Song, D.; Liu, H. Interface for online coupling of surface plasmon resonance to direct analysis in real time mass spectrometry. Anal. Chem. 2015, 87, 6505-6509.

96. Zhang, Y.; Xu, S.; Wen, L.; Bai, Y.; Niu, L.; Song, D.; Liu, H. Dielectric barrier discharge ionization based interface for online coupling surface plasmon resonance with mass spectrometry. Analyst 2016, 141, 3343-3348. 
97. Krone, J. R.; Nelson, R. W.; Dogruel, D.; Williams, P.; Granzow, R. BIA/MS: Interfacing biomolecular interaction analysis with mass spectrometry. Anal. Biochem. 1997, 244, 124132.

98. Urban, P. L.; Amantonico, A.; Zenobi, R. Lab-on-a-plate: Extending the functionality of MALDI-MS and LDI-MS targets. Mass Spectrom. Rev. 2011, 30, 435-478.

99. Takáts, Z.; Wiseman, J. M.; Gologan, B.; Cooks, R. G. Mass spectrometry sampling under ambient conditions with desorption electrospray ionization. Science 2004, 306, 471-473.

100. Nielen, M. W. F.; Hooijerink, H.; Zomer, P.; Mol, J. G. J. Desorption electrospray ionization mass spectrometry in the analysis of chemical food contaminants in food. Trends Anal. Chem. 2011, 30, 165-180.

101. Klampfl, C. W.; Himmelsbach, M. Direct ionization methods in mass spectrometry: An overview. Anal. Chim. Acta 2015, 890, 44-59.

102. Paine, M. R. L.; Barker, P. J.; Blanksby, S. J. Paint spray mass spectrometry for the detection of additives from polymers on conducting surfaces. Mass Spectrom. Lett. 2012, 3, 25-28.

103. Hu, B.; So, P.-K.; Yao, Z.-P. Electrospray ionization with aluminum foil: A versatile mass spectrometric technique. Anal. Chim. Acta 2014, 817, 1-8.

104. Jiang, J.; Zhang, H.; Li, M.; Dulay, M. T.; Ingram, A. J.; Li, N.; You, H.; Zare, R. N. Droplet spray ionization from a glass microscope slide: Real-time monitoring of ethylene polymerization. Anal. Chem. 2015, 87, 8057-8062.

105. Gómez-Ríos, G. A.; Pawliszyn, J. Development of coated blade spray ionization mass spectrometry for the quantitation of target analytes present in complex matrices. Angew. Chem. Int. Ed. 2014, 53, 14503-14507. 


\title{
Chapter 2
}

\section{Surface characterization and antifouling properties of nanostructured gold chips for imaging surface plasmon resonance biosensing}

\author{
Sweccha Joshi, ${ }^{1,2}$, Paola Pellacani ${ }^{3}$, Teris A. van Beek ${ }^{1}$, Han Zuilhof ${ }^{1}$, Michel W.F. Nielen ${ }^{1,4}$ \\ ${ }^{1}$ Laboratory of Organic Chemistry, Wageningen University, Dreijenplein 8, 6703 HB \\ Wageningen, The Netherlands \\ ${ }^{2}$ TI-COAST, Science Park 904, 1098 XH, Amsterdam, The Netherlands \\ ${ }^{3}$ Plasmore S.R.L, Via Deledda 4, 21020 Ranco (VA), Italy \\ ${ }^{4}$ RIKILT Wageningen UR, P.O. Box 230, 6700 AE Wageningen, The Netherlands
}

This chapter has been published in:

Sensors and Actuators, B, 2015, 209, 505-514. 


\section{Abstract}

Surface Plasmon Resonance (SPR) optical sensing is a label-free technique for real-time monitoring of biomolecular interactions. Recently, a portable imaging SPR (iSPR) prototype instrument, featuring a nanostructured gold chip, has been developed. In the present work, we investigated the crucial first steps, prior to eventual use of the nanostructured iSPR chip, i.e., its surface modification, in-depth surface characterization and the antifouling performance. Results were compared with conventional flat (i)SPR gold chips having the same surface chemistries, viz. different types of polyethylene glycol and zwitterionic polymers. Characterization of the (i)SPR chips before and after surface modification was performed using atomic force microscopy (AFM), scanning electron microscopy (SEM), water contact angle (WCA), X-ray photoelectron spectroscopy (XPS) and direct analysis in real time high resolution mass spectrometry (DART-HRMS). The antifouling properties were then studied using the nanostructured chip in the portable iSPR instrument and the flat gold chip in conventional SPR set-up. The zwitterionic polymer surface chemistries showed the best antifouling properties. Comparison of the nanostructured iSPR chips with conventional flat (i)SPR gold chips showed that the latter perform slightly better in terms of surface modification as well as antifouling properties. The portable iSPR instrument is almost as sensitive as conventional iSPR (IBIS) and nine times less sensitive than conventional SPR (Biacore 3000). The nanostructured iSPR chip, along with the portable instrument, offers the advantage of about ten-fold reduction in instrument size, weight and costs compared to conventional (i)SPR instruments using flat gold, thus making it highly interesting for future biosensing applications.

\section{Keywords}

Nanoplasmonics, imaging SPR, surface characterization, zwitterionic polymers, poly(ethyleneglycol), miniaturization 


\section{Introduction}

Surface Plasmon Resonance (SPR) based biosensors have emerged as fast, sensitive, and label-free techniques for real-time monitoring of biomolecular interactions. ${ }^{1,2}$ The desire to measure multiple biointeractions in parallel has triggered the development of a new platform known as imaging SPR (iSPR) ${ }^{2-4}$ In iSPR, the reflected light is collected by a charge-coupled device ( $C C D$ ) camera, which allows real-time visualization of the change in reflectivity at multiple spots on the sensor surface. ${ }^{5}$ Conventional iSPR instruments, although quite successful in biosensing applications, ${ }^{6-8}$ are rather heavy and costly, and should be considered as high-end laboratory-based biosensing equipment. Considering the demand for bringing the lab to the sample, a portable iSPR prototype has been developed recently, ${ }^{9,10}$ which offers the potential for in-field and at-line biosensing applications. Unlike conventional SPR (Figure 1), the miniaturized iSPR instrument uses nanostructured gold instead of flat gold as a sensor chip surface. The nanostructured surface is made up of a periodic alternation of poly (methyl methacrylate) and gold ${ }^{11}$ as shown in Figure $1 \mathrm{~A}$ and 2 . This forms a metal-dielectric pattern that acts as a metallic nanograting, ${ }^{12,13}$ thus eliminating the use of expensive and delicate prism-based optics and contributing to miniaturization, portability and low costs of the instrument. Additionally, the periodicity of a nanostructured gold surface is known to influence the SPR signal. ${ }^{14}$ Due to advantages over flat surfaces, nanostructured gold has also found application in surface-enhanced Raman spectroscopy (SERS). ${ }^{15-17}$

A crucial step towards the use of any sensor chip for biosensor applications, is prevention of non-specific interactions of biomolecules (biofouling) leading to false positive signals. This requires the use of well-defined antifouling chemistries that not only help to overcome fouling issues but also to obtain sufficient surface stability for regeneration and repeated use of the sensor surface. SPR optical sensing has been used extensively to study antifouling properties of chemically modified flat gold. ${ }^{18-22} \mathrm{~A}$ widespread approach to reduce non-specific adsorption is to modify the surface using a coating of hydrophilic polymers. Carboxymethylated (CM) dextran coatings are the oldest and very popular antifouling layers used in commercial SPR sensors. ${ }^{23}$ As an alternative to $C M$ dextran, poly(ethylene glycol) (PEG)-tethered alkanethiol chains, terminated with various functional groups, have been used. ${ }^{20,24}$ PEG is a hydrophilic, electrically neutral polyether known to be resistant to non-specific adsorption mainly due to steric hindrance and water barrier effects. ${ }^{25}$ Careful tuning of the length and density of the PEG-tethered chains is, however, required to achieve the desired effect. ${ }^{26}$ These parameters are dependent on the method of polymer growth, thus it is interesting and relevant to compare "grafting to"27 and "grafting from"28 approaches of polymer attachment. Although PEG has been used extensively, it is known to oxidize easily in the presence of 
oxygen and various transition metal ions. ${ }^{24}$ Recently, interest in finding alternatives for PEG is increasing, leading to the development of a new class of antifouling surfaces composed of zwitterionic polymers. ${ }^{22}$ The antifouling properties of zwitterionic polymer surfaces stem mainly from the strong electrostatic interaction between the opposite charges present and its high hydration capacity. ${ }^{27,29,30}$

In-depth characterization of the sensor chip prior to antifouling experiments is important to understand the surface properties. The nanostructured gold surface used in the present study is an integral part of the design of the portable iSPR setup (Figure $1 \mathrm{~A}$ ). The nanostructured chip in combination with the iSPR instrument offers the advantage of reduced instrument size, weight and costs compared to conventional (i)SPR using flat gold and a prism. The surface modification and subsequent characterization of these nanostructured gold chips has never been reported in the literature. Therefore, properties of nanostructured iSPR chips were studied and compared with those obtained on flat gold chips modified with the same chemistries for future application transferability.

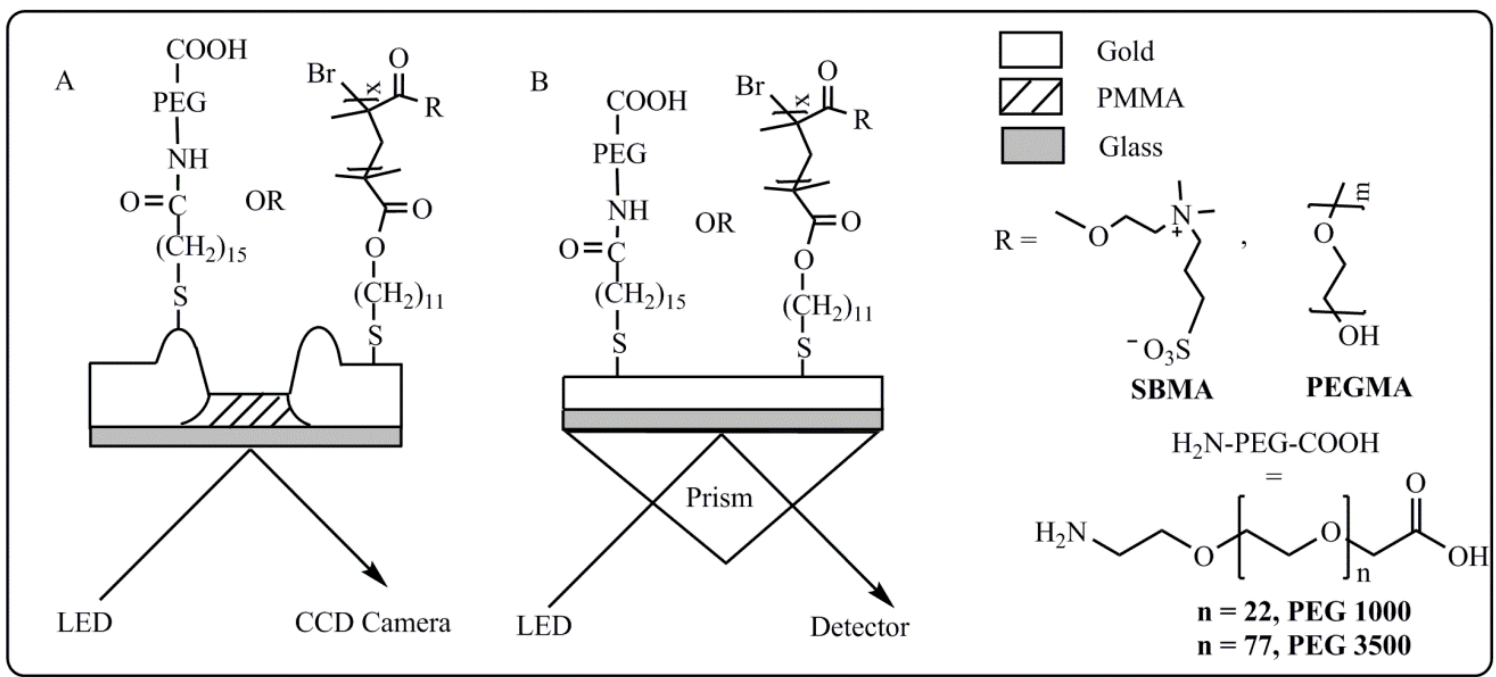

Figure 1. Schematic representation of A) prototype imaging SPR setup with nanostructured gold as sensor surface and B) conventional SPR setup with flat gold as sensor surface, and the antifouling chemistries used.

\section{Materials and methods}

\section{Chemicals and substrates}

16-Mercaptohexadecanoic acid (MHDA), pentafluorophenol (PFP) and $\mathrm{N}, \mathrm{N}^{\prime}-$ dicyclohexylcarbodiimide (DCC), poly(ethylene glycol) 2-aminoethyl ether acetic acid (average MW 1000 and 3500) ( $\mathrm{NH}_{2}$-PEG-COOH), 2,2'-bipyridine (BiPy), anhydrous copper (I) chloride ( $\mathrm{CuCl})$, anhydrous copper (II) chloride $\left(\mathrm{CuCl}_{2}\right)$, (2(methacryloyloxy)ethyl)dimethyl-3-sulfopropyl)ammonium hydroxide (SBMA monomer), 
poly(ethylene glycol) methacrylate (PEGMA monomer, MW 500), isopropanol, bovine serum albumin (BSA) and PBS buffer tablets (1 tablet was dissolved in $200 \mathrm{~mL}$ of deionized water to make $10 \mathrm{mM}$ PBS $\mathrm{pH}$ 7.4) were purchased from Sigma Aldrich (Zwijndrecht, The Netherlands). Absolute ethanol was purchased from Fisher Scientific. All the solvents were used as obtained, except for dichloromethane (Sigma Aldrich, $>99.8 \%$ ), which was further purified using a Pure Solv 400 solvent purification system (Innovative Technology, Amesbury, USA). 11-Mercaptoundec-1-yl 2-bromo-2methylpropionate (Izo-Br) was purchased from Prochimia (Sopot, Poland). HBS-EP buffer (0.01 M HEPES pH 7.4, 0.15 M NaCl, 3 mM EDTA, 0.005\% (v/v) Surfactant P20) and $1 \mathrm{M}$ ethanolamine $\cdot \mathrm{HCl}(\mathrm{pH}$ 8.5) were purchased from GE Healthcare (Diegem, Belgium). Goat milk was bought from a local organic farm. Pale lager (The Netherlands) and Belgian ale were used as beer 1 and beer 2, respectively. Biotin-PEG3-amine and streptavidin were purchased from Thermo Scientific (The Netherlands) and Life Technologies Europe BV (The Netherlands) respectively. Deionized water $(18.3 \mathrm{M} \Omega \cdot \mathrm{cm}$ resistivity) was obtained using a Merck (Amsterdam, The Netherlands) water purification system.

Flat gold substrates of $\mathrm{Au}$ sputtered on glass were purchased from Xantec (Düsseldorf, Germany). The nanostructured gold chips were produced using colloidal lithography and plasma-enhanced chemical vapor deposition (PE-CVD). ${ }^{31}$ Briefly, poly(methyl methacrylate) (PMMA) was deposited on the glass substrate by PE-CVD using methyl methacrylate as liquid precursor. Spin coating was then used to cover the film with polystyrene (PS) beads (500 nm with a nominal size dispersion of $10 \%$ ). Next, the sample was exposed to oxygen plasma etching to remove the PMMA from the areas not covered by the beads and to reduce the size of the PS beads to provide the required periodicity. A gold layer of 100-200 nm was then deposited on the surface by physical vapor deposition. Finally, the polystyrene beads were removed resulting in a surface with a gold film perforated by PMMA (Figure $1 \mathrm{~A}$ and 2 ). The combined control over deposition and etching parameters allowed fine tuning of film thickness and PMMA well diameters.

\section{Surface Modification}

"Grafting to" growth of polyethylene glycol. Prior to modification, the Au substrates (flat and nanostructured) were rinsed with ethanol and water followed by drying with nitrogen. The chips were then immersed in a $1 \mathrm{mM}$ solution of 16 -mercaptohexadecanoic acid (MHDA) in ethanol for $24 \mathrm{~h}$. The MHDA-modified surfaces were removed and rinsed with ethanol and water, sonicated in ethanol, and dried with nitrogen. The acidterminated substrates were activated by immersing overnight in a $1: 1$ mixture of $0.4 \mathrm{M}$ PFP and 0.4 M DCC in dichloromethane. ${ }^{32}$ The PFP-terminated substrates were washed with DCM and then immersed in a $1 \mathrm{mg} / \mathrm{mL}$ solution of $\mathrm{NH}_{2}-\mathrm{PEG}-\mathrm{COOH}$ in DCM for $24 \mathrm{~h}$. The PEG-modified (PEG1000 and PEG3500) surfaces were removed and rinsed with DCM, 
sonicated in the same solvent, and dried with nitrogen. Any remaining PFP activated esters were deactivated by immersion in ethanolamine for 30 min prior to the antifouling experiments.

"Grafting from" growth of polyethylene glycol and zwitterionic polymers. The Au substrates were coated with an atom transfer radical polymerization (ATRP) initiator by immersing them in $2.5 \mu \mathrm{L} / \mathrm{mL}$ solution of Izo- $\mathrm{Br}$ in absolute ethanol at room temperature for $24 \mathrm{~h}$. The cleaning procedures before and after modification were the same as in the case of MHDA. For the polymerization, a procedure from literature was used. ${ }^{33}$ SBMA monomer $(4.90 \mathrm{~g}, 17.5 \mathrm{mmol})$ and BiPy $(0.14 \mathrm{~g}, 0.9 \mathrm{mmol})$ were dissolved in deionized water $(16 \mathrm{~mL})$ and isopropanol $(4 \mathrm{~mL})$. The solution was then degassed by purging with argon for $30 \mathrm{~min}$. $\mathrm{CuCl}(71.3 \mathrm{mg}, 0.72 \mathrm{mmol})$ and $\mathrm{CuCl}_{2}(9.7 \mathrm{mg}, 0.072 \mathrm{mmol})$ were weighed in a separate flask inside a glove box (Argon 6.0, $\mathrm{O}_{2}<0.1 \mathrm{ppm}$, and $\mathrm{H}_{2} \mathrm{O}<0.1$ $\mathrm{ppm})$. The flask was sealed with a rubber septum to ensure an oxygen-free environment. The SBMA monomer-BiPy solution was then transferred to the flask containing $\mathrm{CuCl} / \mathrm{CuCl}_{2}$ using an argon-flushed double-tipped needle. The solution was stirred under argon for $30 \mathrm{~min}$ to completely dissolve the $\mathrm{CuCl} / \mathrm{CuCl}_{2}$. Meanwhile, the initiator-coated gold substrate was placed in a separate flask and flushed with argon. The mixture containing monomer, $\mathrm{BiPy}$ and $\mathrm{CuCl} / \mathrm{CuCl}_{2}$ was transferred to the flask with the initiatorcoated substrate, again using an argon-flushed double-tipped needle. Next, the substrate was left to polymerize under argon at room temperature for $2 \mathrm{~h}$. Finally, the SBMAmodified surface was rinsed with water, sonicated in water and dried with nitrogen. The same procedure was used for PEGMA using PEGMA monomer $(8.75 \mathrm{~g}, 17.5 \mathrm{mmol})$. The PEGMA monomer was passed through a column of activated, basic aluminum oxide directly prior to the polymerization to remove inhibitors. In case of PEGMA, the substrates were additionally rinsed with and sonicated in methanol to remove any remaining monomer.

In the case of nanostructured gold, the chip surface was modified only partially, in order to have an unmodified gold region as a reference on the same chip. The possibility of having a reference inside the same chip helps to correct for any fluctuations caused by differences in illumination of the surface. Simple dipping of (a part of) the chip did not work, due to capillary forces acting on the solvent (mainly ethanol). Therefore, an oval PEEK flow-cell (internal dimension: $18 \mathrm{~mm} \times 4 \mathrm{~mm}$, volume: $4 \mu \mathrm{L}$ ) sealed by Viton $\mathrm{O}-$ rings (see Figure S1) was used, providing both resistance to the solvents (ethanol and dichloromethane) as well as a good sealing. Using this flow-cell the nanostructured gold was modified with MHDA, PFP, PEG1000, PEG3500 and Izo-Br by flowing the respective solution as mentioned above at a continuous flow rate of $0.1 \mu \mathrm{L} / \mathrm{min}$ over a part of the chip surface. After the partial modification of the surface with Izo-Br, subsequent 
reactions with SBMA and PEGMA had to be performed under argon and the use of the flow-cell setup was not feasible. Fortunately, in these cases, the solvent was mainly water, thus simple dipping was feasible at this stage to obtain a partially modified surface. The chips were placed in narrow glass tubes and the solution was carefully transferred to fill the tube from the bottom until part of the chip was covered. It was important that the nanostructured chip was only partially submerged, as physisorption of the polymer, although to a lesser extent (as measured by XPS) than the part with initiator, was observed also in the areas without the initiator.

\section{Surface Characterization}

Atomic Force Microscopy (AFM). The AFM images were taken using a JSPM-5400 (Jeol, Japan) Scanning Probe Microscope in AC-AFM ("tapping") mode, and OMCLAC240TS-R3 (Olympus, Japan) cantilevers.

Scanning Electron Microscopy (SEM). The SEM images were taken using a JAMP9500F (Jeol, Japan) Field Emission Auger Microscope. The nanostructured surface was placed in a JFC-1300 (Jeol, Japan) auto fine coater for $40 \mathrm{~s}$ to obtain a thin layer of gold that reduces charging effects during SEM measurements, and helps to get a good focusing.

Static water contact angle measurements. The wettability of the surfaces was determined by measuring the static water contact angle (WCA) using a DSA-100 (Krüss, Germany) goniometer. Drops ( 2-5 depending on the sample size) of $3 \mu \mathrm{L}$ of MilliQ water were dispensed on Au surfaces with a microliter syringe with stainless steel needle (diameter $=0.51 \mathrm{~mm}$ ), and the CA was determined using a Tangent 2 fitting model.

X-ray Photoelectron Spectroscopy (XPS). XPS analyses of the surfaces were performed using a JPS-9200 (Jeol, Japan) photoelectron spectrometer. The spectra were obtained under UHV conditions using monochromatic Al Ka X-ray radiation at $12 \mathrm{kV}$ and $20 \mathrm{~mA}$, using analyzer pass energy of $10 \mathrm{eV}$. The spectra were reprocessed using the CASA XPS peak fit program (version 2.3.16). The curve fitting was performed with a linear background fitting. The thickness of the organic layers on the surface was calculated using the following formula:

$\mathrm{t}=\ln \frac{\mathrm{I}_{\mathrm{Auo}}}{\mathrm{I}_{\mathrm{Au}}} \times \lambda_{\mathrm{Au}} \times \cos \theta^{34}$

where $t=$ thickness (in $\mathrm{nm}$ ) of the monolayer, $I_{\text {Auo }}=$ intensity of XPS signal of Au4f $\mathrm{f}_{7 / 2}$ at $83.9 \mathrm{eV}$ (relative to C1s signal) in unmodified gold, $I_{A u}=$ intensity of XPS signal of Au4f $f_{7 / 2}$ (relative to $\mathrm{C} 1 \mathrm{~s}$ signal) in modified gold, $\lambda_{\mathrm{Au}}=$ effective attenuation length of Au4f electrons in the organic films (using a value of $3.858 \mathrm{~nm}$ as reported by Petrovykh et al. 
${ }^{35}$ for DNA films), $\theta$ = the photoelectron emission takeoff angle relative to the surface normal $\left(10^{\circ}\right)$.

\section{Direct Analysis in Real Time High-Resolution Mass Spectrometry (DART-HRMS).} The DART-orbitrap high-resolution mass spectrometer system consisted of a DART-SVP ion source (Ion-Sense, Saugus, USA) coupled to an Exactive high resolution MS system (Thermo Fisher Scientific, San Jose, CA, USA). The MS was calibrated daily using ProteoMass $^{\mathrm{TM}}$ LTQ/FT-Hybrid ESI (positive and negative mode) Cal Mix (Sigma Aldrich), which is applicable for the $\mathrm{m} / \mathrm{z}$ range 100-2000. XCalibur software (version 2.1) was used for instrument control, data acquisition and data processing. For the DART, helium (flow of $~ 3.5 \mathrm{~L} / \mathrm{min}$ ) was used as ionizing gas, with the gas beam temperature set at $400^{\circ} \mathrm{C}$. The (i)SPR chips (blank and modified) were attached to a glass slide using double-sided tape. The glass slide was then fixed onto a motorized rail placed between the DART source and MS inlet. The DART was pointed at an angle of $45^{\circ}$ above the chip surface. A detailed description of the technique of surface measurements can be found in a previous publication. ${ }^{36}$ The neat solutions were measured by placing a drop of the solution onto a glass slide using the same settings as for modified samples. All the surfaces were measured in positive ion mode. The surfaces modified with PFP were additionally characterized in negative ion mode.

\section{Antifouling experiments}

BSA, beer and milk samples were used to test the antifouling properties of different surface chemistries. BSA was chosen as a general model protein for non-specific adsorption, while beer and milk were chosen as model sample matrices for real-life SPR applications. SPR measurements on flat gold were performed using a Biacore 3000 (GE Healthcare, Sweden), and on nanostructured gold using an imaging nanoplasmonics instrument (Plasmore Srl., Italy). Prior to the antifouling experiments, the chips were washed for $1 \mathrm{~min}$ with $5 \mathrm{mM} \mathrm{NaOH}$ followed by $3 \mathrm{~min}$ ( $t=0-180 \mathrm{~s}$ ) with running buffer (HBS-EP) to get a stable baseline. The non-specific binding was monitored by injecting BSA (1 mg/mL in HBS-EP), beer samples (four times diluted in HBS-EP for Biacore and undiluted for nanostructured gold), and milk (10\% diluted in HBS-EP) at a constant flow of $20 \mu \mathrm{L} / \mathrm{min}$. After a $5 \mathrm{~min}(\mathrm{t}=180-480 \mathrm{~s})$ injection of the analyte, the channels were washed with running buffer (HBS-EP). The response obtained after $4 \min (t=720 \mathrm{~s})$ of buffer flow ( $20 \mu \mathrm{L} / \mathrm{min})$, relative to the starting baseline, was used as a measure of the amount of fouling. For comparing the fouling from different samples on the different modified gold surfaces (flat and nanostructured), the relative response obtained was normalized to the response obtained for bare gold upon addition of $1 \mathrm{mg} / \mathrm{mL}$ BSA to the respective gold surface. The sensorgrams and bar graphs were plotted using Origin 8.5 
and Excel 2010. Sensorgrams from the portable iSPR instrument were smoothened using the Savitzky-Golay function in Origin 8.5 (10 points of window, polynomial order 2).

Sensitivity measurement: The sensitivity of the portable iSPR instrument was compared to that of conventional SPR (Biacore 3000) and imaging SPR (IBIS Technologies B.V., Hengelo, The Netherlands). Calibration curves were constructed with serial dilutions of glycerol ranging from 10 to $0.01 \%$ for each instrument using the respective bare chips. The LODs were calculated as three times the standard deviation of the baseline (noise). To allow comparison of the different instruments, each with their own units of response, the LODs were converted to percentage of glycerol using the fitting equation of the calibration curve.

Biotin-streptavidin binding: PEG3500 modified chips were activated using a 1:1 mixture of $0.4 \mathrm{M}$ EDC and $0.1 \mathrm{M} \mathrm{NHS}$ for $10 \mathrm{~min}$. The chips were washed with water and then covered with a $1 \mathrm{mg} / \mathrm{mL}$ solution of biotin-PEG-NH $\mathrm{NH}_{2}$ in PBS ( $\left.\mathrm{pH} 7.4\right)$ for $2 \mathrm{~h}$ at room temperature. The chips were then washed with PBS and subsequently blocked with ethanolamine for $10 \mathrm{~min}$. The modifications with biotin were confirmed by XPS. The chips were then mounted on the corresponding instruments to monitor the binding of streptavidin (Figure 6 and S16). A $10 \mu \mathrm{g} / \mathrm{mL}$ solution of streptavidin (diluted in HBS-EP) was introduced $(t=50 \mathrm{~s}$ ) at a flow rate of $20 \mu \mathrm{L} / \mathrm{min}$. After a $5 \mathrm{~min}(t=50-350 \mathrm{~s})$ injection, the chips were washed with running buffer (HBS-EP). The response obtained after $30 \mathrm{~s}(\mathrm{t}=380 \mathrm{~s})$ of buffer flow $(20 \mu \mathrm{L} / \mathrm{min})$, relative to the starting baseline, was used as a measure of the amount of binding.

\section{Results and discussion}

\section{Surface modification and characterization}

AFM and SEM were used to investigate the surface morphology of the nanostructured iSPR chip. Both techniques clearly display the nanograting with periodic alternation of gold and poly(methyl methacrylate) (see Figure 2). Three regions can be seen as indicated by the arrows in Figure 2: flat gold, poly(methyl methacrylate) (PMMA) wells, and gold rings surrounding the PMMA. The poly(methyl methacrylate) regions with diameters of 200-250 nm and periodicity (distance between the centers of the two PMMA wells) of $500-600 \mathrm{~nm}$ make up around $20 \%$ of the total surface area. The height of the gold rings is in the range of $10-20 \mathrm{~nm}$. The surface contains some defects and residual polystyrene beads as seen in Figure $2 \mathrm{~B}$. However, these defects and residual PS beads are minor, and most likely do not affect the overall modification, antifouling and sensing properties of the chips in a significant fashion. 


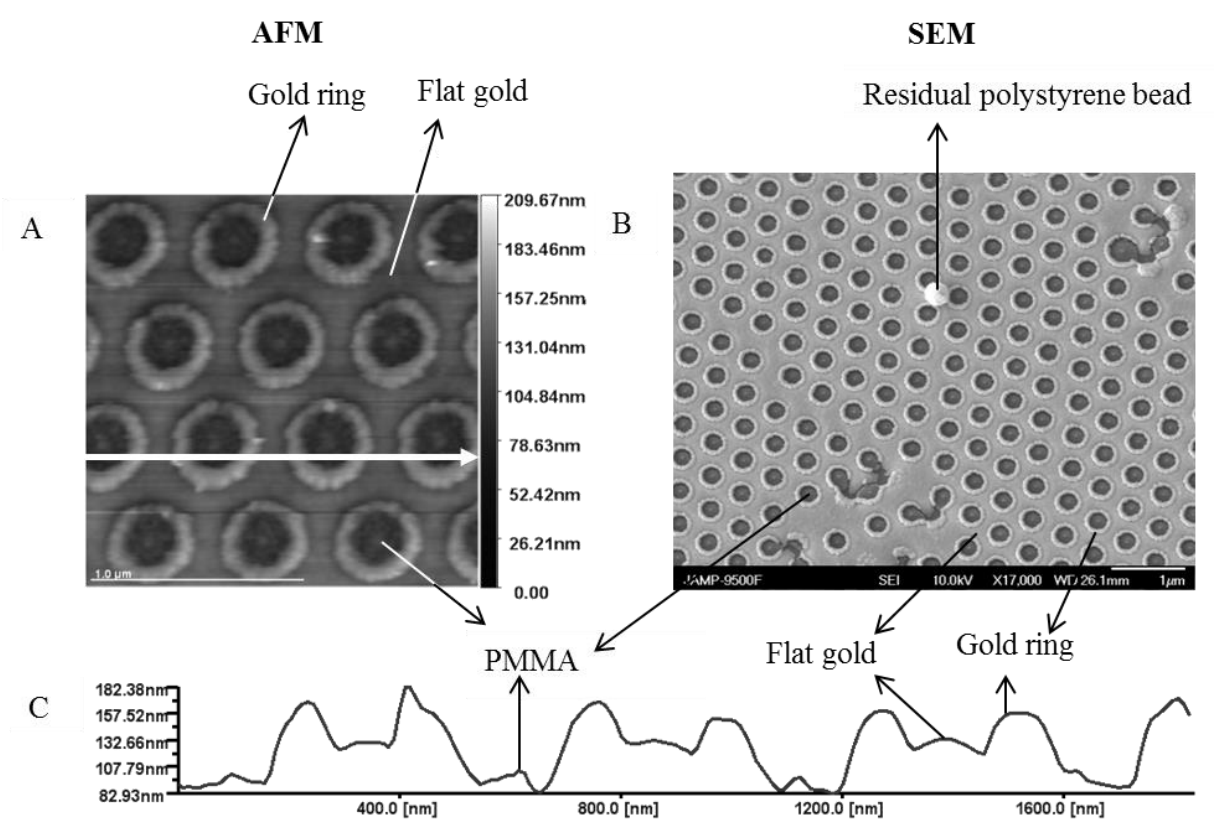

Figure 2. Surface characterization of unmodified nanostructured Au iSPR chip. A) AFM, B) SEM images, and C) AFM profile of the surface along the horizontal arrow in A.

Since, the chemical modification of these nanostructured iSPR substrates has not been studied before, each step of the surface modification was carefully characterized using water contact angle measurements, X-ray photoelectron spectroscopy and DARTHRMS and the data were compared to those of flat SPR surfaces modified according to the same procedures. The contact angles of bare gold, MHDA, PEG1000 and PEG3500 modified nanostructured gold (Table 1 ) were found to be significantly higher than those measured on flat gold (Table 1) and reported for flat gold $\left(65^{\circ}, 44^{\circ}, 42^{\circ}\right.$ and $40^{\circ}$, respectively). ${ }^{27}$ The contact angle values for PEG are difficult to compare with literature values as small differences in these multistep surface modifications may lead to differences in contact angle values. Such variations, also reported in the literature, ${ }^{37}$ may arise due to differences in cleaning procedure, nature of initial self-assembled monolayer and differences in polydispersity, purity of end groups and average molecular weight of the PEG depending on the supplier. The higher values for nanostructured gold are probably due to the PMMA regions, which are expected to have a high contact angle $\left(80^{\circ}\right.$ $\left.90^{\circ}\right){ }^{38}$ With a surface area of $20 \%$, PMMA can significantly affect the contact angle in case of thin layers. Note that the PMMA surface may be converted, at least partially, into a carboxylic acid surface during the plasma treatment step in the manufacturing of the nanostructured chips. But any remaining hydrophobic PMMA regions will contribute to the overall contact angle. The contact angles values of PFP and Izo-Br modified nanostructured gold are in close agreement with the observed (Table 1 ) and reported values for similarly modified flat gold surfaces $\left(90^{\circ}\right.$ and $80^{\circ}$, respectively). ${ }^{39}$ These contact angle values are already close to that of PMMA and thus will not be much 
affected. The effect of PMMA regions becomes insignificant in the case of successful modification with thicker polymers, such as SBMA and PEGMA, where the contact angles of modified nanostructured gold are in good agreement with those of modified flat gold (Table 1 ) and previous studies $\left(20^{\circ}\right.$ and $47^{\circ}$, respectively). ${ }^{37}$

XPS was used to determine the elemental composition of the organic layer, and also the dry thickness thereof, as determined from the attenuation of the Au4f $f_{7 / 2}$ signal from the bare gold surface to the coated ones. ${ }^{35}$ The relative atomic percentages of different elements (Figure S2-S4) and the layer thickness (Table 1) as calculated from an XPS survey scan for MHDA, PFP and Izo-Br for both flat and nanostructured gold, are in agreement with the expected values of $2.1 \mathrm{~nm}, 2.3 \mathrm{~nm}$, and $1.8 \mathrm{~nm}$, respectively. Typically, in the C1s narrow scan of these monolayers the peak at $286.5 \mathrm{eV}$ is slightly broader and shifted by $1 \mathrm{eV}$ for the nanostructured iSPR chip relative to flat gold. This is probably due to the underlying PMMA layer that can still be measured by XPS in case of thin layers $(<10 \mathrm{~nm})$. For PEG1000 and PEG3500 modified nanostructured gold, the dry thickness calculated from the XPS survey scan is lower than that on flat gold (roughly 2 $\mathrm{nm}$ versus $4 \mathrm{~nm}$, respectively). According to the literature, the thickness of "grafted to" PEG is expected to be less than $5 \mathrm{~nm} .{ }^{37,40}$ Under the conditions of XPS, these layers are in a collapsed state and the effect is more pronounced in the case of nanostructured gold. This is possibly due to the underlying PMMA that obstructs the formation of a well packed monolayer which, in turn, translates to a lower "grafting to" efficiency in case of the nanostructured gold. Nevertheless, the increased intensity of the carbon peak at $286.7 \mathrm{eV}$ in the C1s spectrum, corresponding to carbon attached to oxygen (Figure S5 and S6), does confirm the surface attachment of polyethylene glycol units, however to a slightly lower extent compared to flat gold. The presence of PEG units on the surface was also confirmed by the representative ion series observed in DART (see further below). The peak at $285 \mathrm{eV}$ in the $\mathrm{C} 1 \mathrm{~s}$ spectrum would be expected to be lower than observed for both flat and nanostructured gold. This, along with a slightly higher $\mathrm{C}$ content from the survey scan $(C / O=2.5: 1)$ and the contact angle values, suggest an effect of PMMA on the thin layers formed. In case of PEGMA (Figure $3 \mathrm{~A}$ and S7), a thick layer is formed on both nanostructured and flat gold as seen from the high $\mathrm{C}$ and $\mathrm{O}$ content in the survey scan and a significant peak at $286.7 \mathrm{eV}$ (C1s narrow scan). The dry layer thickness of PEGMA and SBMA modified nanostructured gold, 11 and $14 \mathrm{~nm}$, respectively, are slightly lower than that of correspondingly modified flat gold, (15 and $21 \mathrm{~nm}$, respectively, Table 1). In case of SBMA-modified surfaces (Figure $3 B$ and $S 8$ ), the relative percentage of the different elements are in good agreement with expected values for the monomers, and the ratios of the carbon peaks ( $\mathrm{C} 1 \mathrm{~s}$ narrow scan) at $285 \mathrm{eV}$ and $286.8 \mathrm{eV}$ are as expected almost equal. In line with reports on SBMA-modified silicon nitride surfaces, ${ }^{41}$ this indicates the formation of significant ( $>10 \mathrm{~nm}$ ) polymer films. 
Table 1. Comparison of water contact angle, dry layer thickness determined by XPS, and representative ions measured in DART-HRMS (measured in positive ion mode, unless otherwise mentioned) with the corresponding $\mathrm{m} / \mathrm{z}$ values for different modification on flat and nanostructured gold. ${ }^{a}$

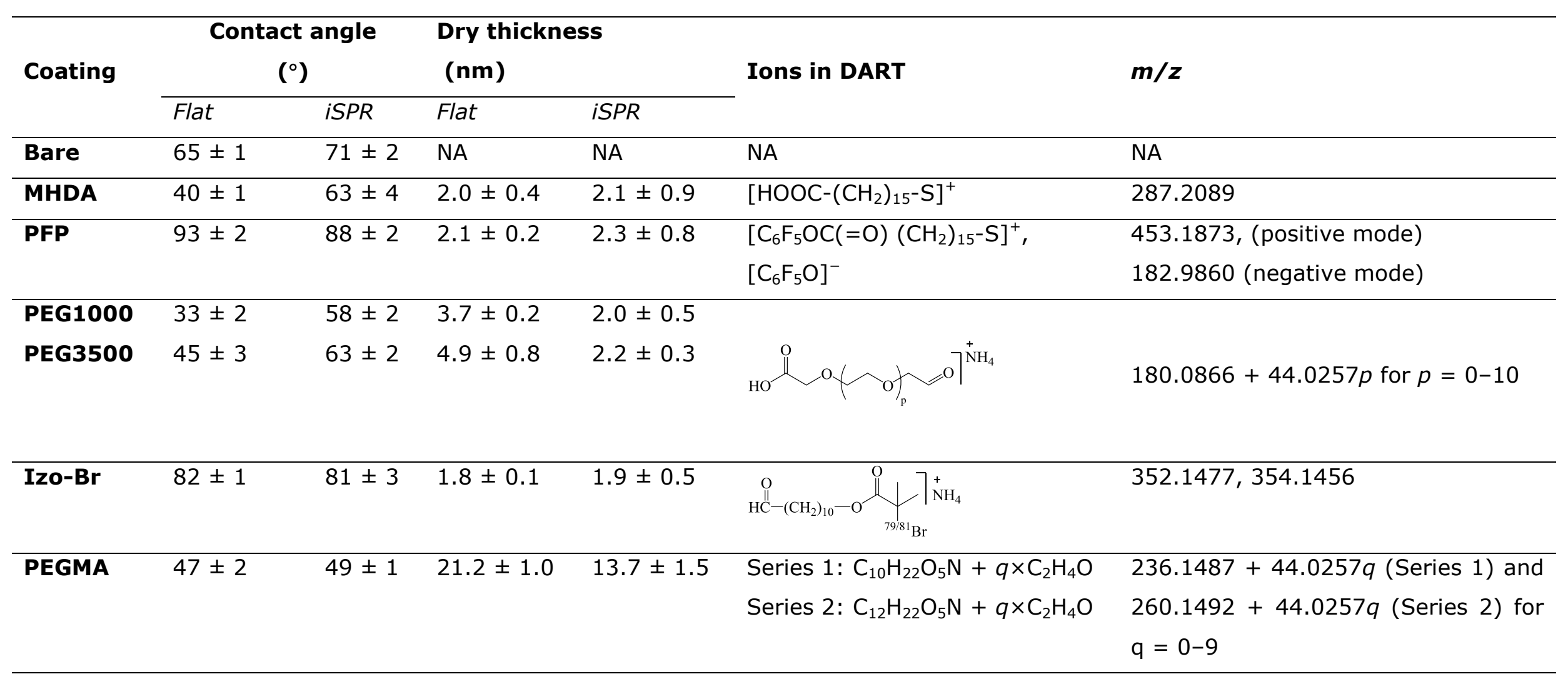

$\begin{array}{lllll}\text { SBMA } & 21 \pm 3 & 24 \pm 2 & 14.9 \pm 1.8 & 11.1 \pm 2.1\end{array}$

${ }^{a}$ The values reported in the table are an average of three measurements and the errors are represented as the standard deviation of the three measurements. NA - not applicable. 

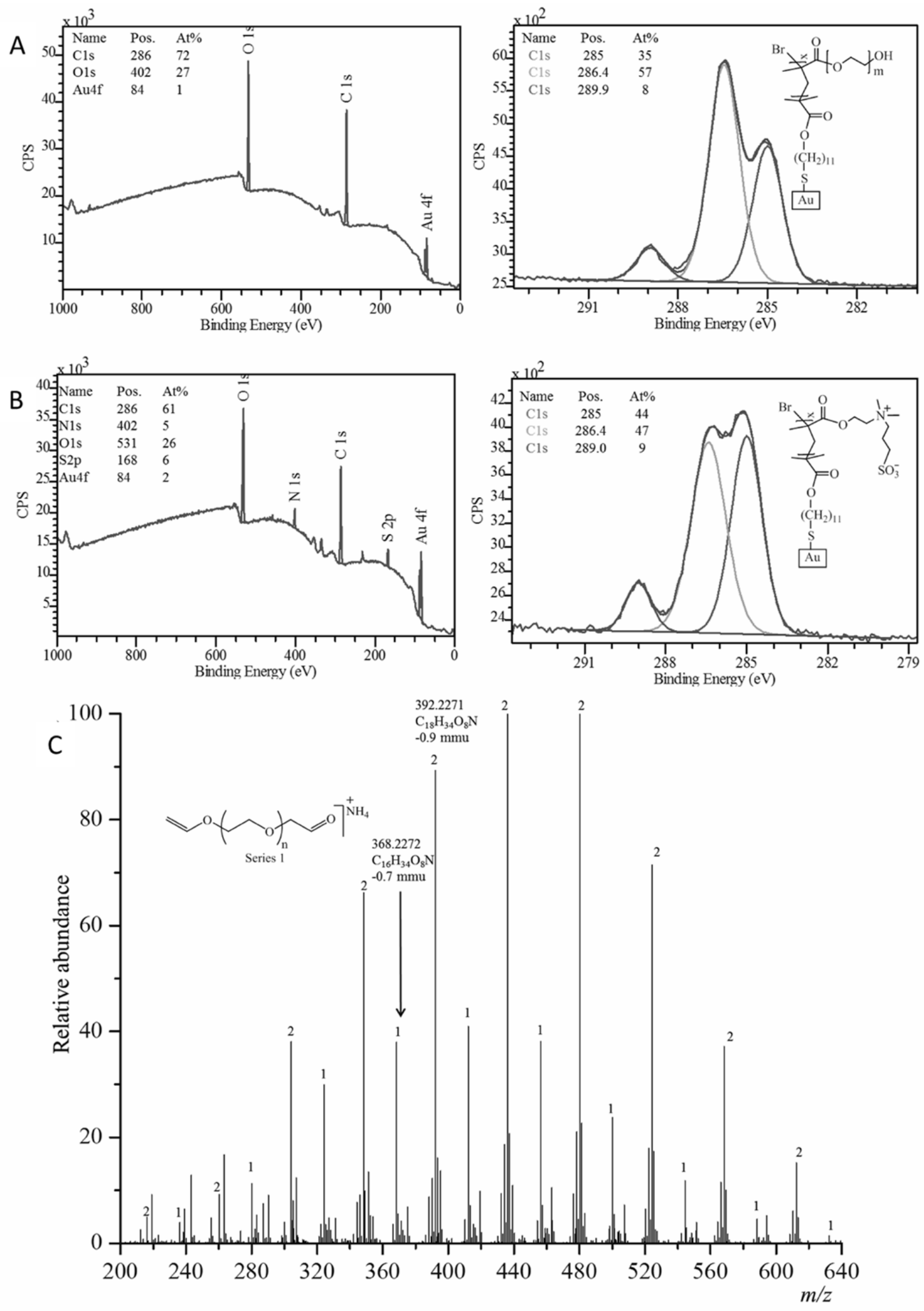

Figure 3. Surface characterization of modified nanostructured gold. XPS survey scan (left) and C1s narrow scan (right) of A) PEGMA-modified, and B) SBMA-modified nanostructured gold, and C) direct analysis in real time high resolution mass spectrum of PEGMA-modified nanostructured gold from $m / z$ 170-670. The two abundant ion series of oligoethylene oxide chains are marked with 1 and 2 respectively. 
DART-HRMS is a powerful tool for surface analysis complementary to $X P S,{ }^{42}$ and was applied here to both flat and nanostructured chip surfaces. In all cases, the same ions were observed, although sometimes with different relative intensities. Ions of the intact molecule were observed for small molecules, such as MHDA ([HOOC- $\left.\left.\left(\mathrm{CH}_{2}\right)_{15}-\mathrm{S}\right]^{+}\right)$, and PFP $\left(\left[\mathrm{C}_{6} \mathrm{~F}_{5} \mathrm{OC}(=\mathrm{O})\left(\mathrm{CH}_{2}\right)_{15}-\mathrm{S}\right]^{+}\right)$resulting from the scission of the relatively weak Au-S bond (Table 1), in agreement with literature for self-assembled thiol-on-gold monolayers. ${ }^{43}$ Furthermore, ions after loss of $\mathrm{H}_{2}$ from the intact molecule were also observed in both cases. In the case of MHDA, we also analyzed the molecule on a glass surface by placing a $5 \mu \mathrm{L}$ drop of $1 \mathrm{mg} / \mathrm{mL}$ methanolic solution and the most intense ion observed was the ammonia adduct $\left[\mathrm{HOOC}-\left(\mathrm{CH}_{2}\right)_{15}-\mathrm{SH}+\mathrm{NH}_{4}\right]^{+}$, which was completely absent on the MHDA-modified gold surface (Figure S9). This clearly indicates the absence of unbound MHDA in the modified Au substrates. The PFP-modified surface, when additionally analyzed by DART-HRMS in negative ion mode, showed the ion $\left[\mathrm{C}_{6} \mathrm{~F}_{5} \mathrm{O}\right]^{-}$as a result of in-situ ester hydrolysis (Figure S10), giving rise to the free alcohol. ${ }^{42}$ In case of PEG1000 and PEG3500-modified gold, we observed ammonia adducts of fragments formed by scission of the $\mathrm{C}-\mathrm{O}$ bonds from the carboxyl end of the polymer chain: [HOOC $\left.-\mathrm{CH}_{2} \mathrm{O}\left(\mathrm{CH}_{2} \mathrm{CH}_{2} \mathrm{O}\right)_{\mathrm{p}} \mathrm{CH}_{2} \mathrm{CHO}+\mathrm{NH}_{4}\right]^{+}$(Table 1, Figure $\mathrm{S} 11$ and $\mathrm{S} 12$ ). Fragments with up to 12 ethylene glycol units were observed. Higher mass fragments are probably difficult to be thermally desorbed by DART and were thus not observed. The ammoniated fragments could be easily distinguished from the unbound PEG (Figure S11C), where the most abundant fragments were those representing the protonated primary amine end of the polymers: $\left[\mathrm{HO}-\mathrm{CH}_{2} \mathrm{CH}_{2}\left(\mathrm{OCH}_{2} \mathrm{CH}_{2}\right)_{\mathrm{p}} \mathrm{OCH}_{2} \mathrm{CH}_{2}-\mathrm{NH}_{3}\right]^{+}$. These fragments with a protonated primary amine end were not present on the surfaces modified with PEG1000 and PEG3500. For Izo-Br modified gold (Figure S13), the ammonia adduct of the aldehyde, formed as a result of oxidation of the molecule, was observed: $\left[\left(\mathrm{CH}_{3}\right)_{2} \mathrm{Br}-\mathrm{C}-\right.$ $\left.\mathrm{C}(=\mathrm{O}) \mathrm{O}\left(\mathrm{CH}_{2}\right)_{10}-(\mathrm{C}=\mathrm{O}) \mathrm{H}+\mathrm{NH}_{4}\right]^{+}$(Table 1$)$. The Izo- $\mathrm{Br}$ molecule analyzed on glass $(5 \mu \mathrm{L}$ drop of $1 \mathrm{mg} / \mathrm{mL}$ methanolic solution) showed only the ammonia adduct of the intact molecule $\left[\left(\mathrm{CH}_{3}\right)_{2} \mathrm{Br}-\mathrm{C}-\mathrm{C}(=\mathrm{O}) \mathrm{O}\left(\mathrm{CH}_{2}\right)_{11}-\mathrm{SH}+\mathrm{NH}_{4}\right]^{+}$. In both surface and solution measurements, the isotope pattern confirmed the presence of bromine. In the case of PEGMA-modified gold (Figure $3 \mathrm{C}$ ), several ion series containing ethylene glycol units were seen. Series 1 with a starting composition of $\mathrm{C}_{10} \mathrm{H}_{22} \mathrm{O}_{5} \mathrm{~N}$ can be explained by two possible structures, one after loss of water from the ions formed by scission of the $\mathrm{C}-\mathrm{O}$ bond and the other due to scission of the $\mathrm{C}-\mathrm{O}$ bond from the monomer itself. In fact the same ion series is present in solution (Figure S14), thus making it difficult to distinguish between unbound PEGMA monomer and polymerized PEGMA. However, PEGMA monomer placed on the gold surface with initiator could be completely removed after washing with water and methanol followed by sonication in methanol (Figure S14C). The same procedure was unable to remove the bound polymerized PEGMA. This proves that the ion 
series observed for polymerized PEGMA is not due to unbound monomer. Series 2 with a starting composition of $\mathrm{C}_{12} \mathrm{H}_{22} \mathrm{O}_{5} \mathrm{~N}$ can be due to dimers of PEGMA. Series 2 was present in both PEGMA modified flat (Figure S14A) and nanostructured gold (Figure 3C), however the intensity was much lower in case of flat gold. Several other ion series, besides Series 1 and 2 depicted in Figure 3C, were present in case of PEG1000, PEG3500 and PEGMA, but could not be associated yet with a particular structure. Surfaces modified with SBMA were also studied using DART, but the results are not included in Table 1, as the ions observed could not be related to a particular (sub)structure of SBMA. Probably, only thermal decomposition product ions are generated from SBMA upon DART ionization.

\section{Antifouling experiments}

The antifouling behavior of the surfaces was evaluated using the (i)SPR instruments and BSA, beer and milk as sample matrices (Figure 4). In case of BSA and beer, as expected, the largest amount of biofouling was seen for bare gold (Figure 5). Mercaptohexadecanoic acid is only slightly better, due to its amphiphilic nature. In the case of MHDA-modified nanostructured gold, the underlying PMMA makes the surface more hydrophobic compared to flat $\mathrm{Au}$, thus resulting in still significant fouling upon treatment with BSA. The PEG-modified surfaces, with internal hydrophilicity in addition to the terminal hydrophilicity, showed better antifouling properties than MHDA. The surfaces with PEG1000, both for flat and nanostructured gold, have showed better antifouling properties than MHDA but were not able to completely resist fouling by beer. The surfaces modified with PEG3500, SBMA and PEGMA showed very good antifouling behavior, both towards BSA and beer. The responses obtained for chips having these three surface chemistries, for both flat and nanostructured gold, were similar within the measurement error range. This demonstrates the transferability of the chemistries to the nanostructured gold in terms of antifouling behavior. The difference between PEG1000 and PEG3500 highlights the importance of chain length for effective antifouling surfaces. The best antifouling performance was observed for SBMA. This zwitterionic layer is wellknown to be resistant to fouling due to the strong electrostatic interaction between the charged groups and the water molecules, thus forming a strongly bound hydration layer compared to the one formed via hydrogen bonding in case of PEG. ${ }^{18}$ However, in the case of $10 \%$ milk, fouling of the nanostructured surface was high and did not improve very much; even SBMA was unable to resist non-specific adsorption (Figure S15). Milk can be considered a worst-case sample matrix consisting of fats $(3.7 \%)$, proteins $(3.4 \%)$, lactose $(4.8 \%)$ and minerals $(0.7 \%) .{ }^{44}$ Hydrophobic regions in milk proteins (casein and whey proteins) and long alkyl chains of fats (fatty acids and triacylglycerols) could have a high affinity for the underlying hydrophobic PMMA in case of nanostructured gold. The casein proteins also form micelles consisting of thousands of protein molecules that could 
contribute to further fouling. Further investigations are needed to elucidate why even SBMA-modified nanostructured gold in the portable setup is performing less well for milk versus flat gold in a conventional SPR set-up.
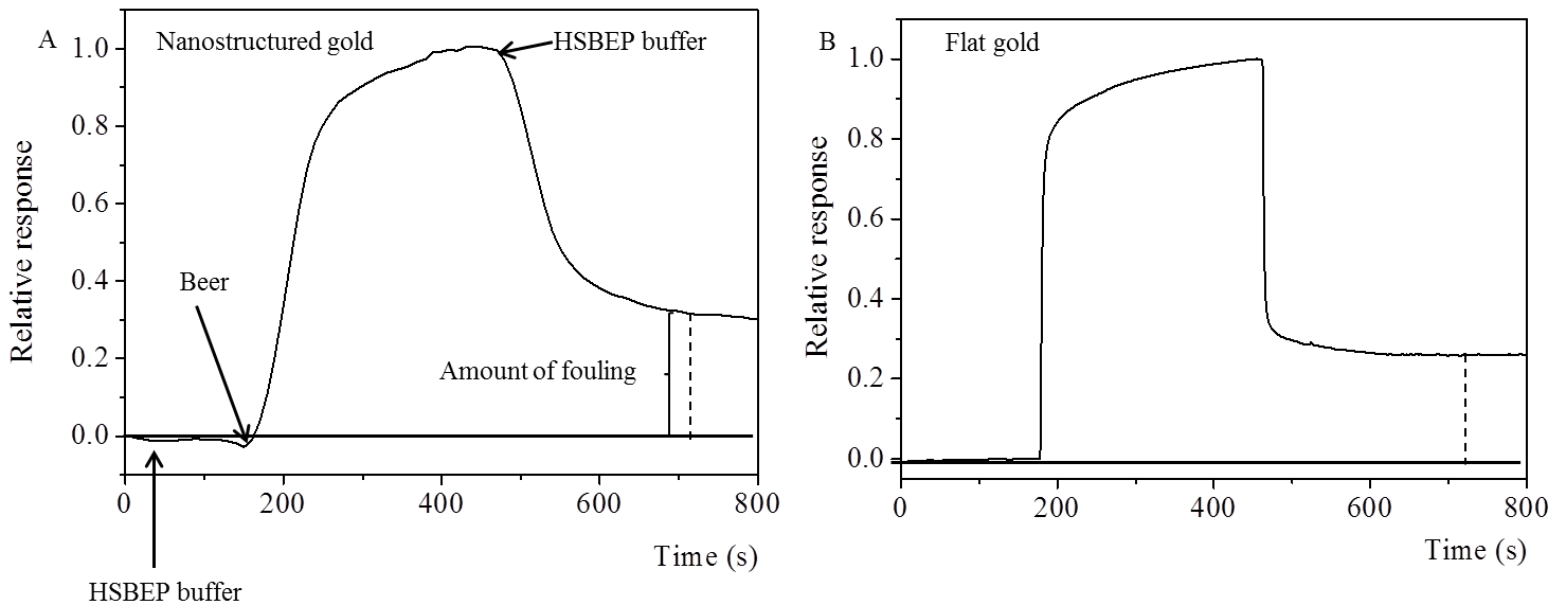

Figure 4. SPR sensorgrams generated upon addition of beer 1 by A) iSPR instrument using bare nanostructured gold, and B) Biacore 3000 using bare flat gold. The vertical dashed line indicates the time point where the relative response was measured to obtain the amount of fouling.

\section{Discussion}

Comparison of contact angle, XPS and DART-HRMS data shows that surface modification of nanostructured gold works analogously to that of flat gold, although the surface coatings are slightly less effective. This was expected, given that $20 \%$ of the surface is covered with PMMA. The conditions for surface modification may be further optimized depending on specific future applications. In terms of instrument performance, the refractive index sensitivity of the portable instrument (LOD $=0.09 \%$ ) is comparable to that of a commercial imaging SPR instrument (IBIS, LOD $=0.07 \%$ ), but almost nine times less sensitive than conventional SPR (Biacore 3000, LOD $=0.01 \%$ ). Preliminary regeneration experiments were performed using sodium hydroxide to test the stability of the nanostructured chip. Upon injecting the same beer sample to a PEG3500 modified chip for another cycle, similar responses were measured (within the measurement error) as the previous cycle (Figure S17). Additionally, a proof-of-principle experiment was performed using biotin-streptavidin as a model system. With both instruments, upon addition of streptavidin to a surface locally modified with biotin, a response was observed only in regions modified with biotin (Figure 6 and Figure S16). The detection of a relatively small protein such as streptavidin shows that the nanostructured surfaces with appropriate surface chemistries are promising for biosensing purposes. As expected due to the strong binding of biotin-streptavidin, the response still remained high during the dissociation phase. The signal to noise of the portable iSPR instrument $(S / N=0.85)$ was 
almost nine times less than that of conventional SPR (Biacore 3000, $S / N=7.9$ ). However, the portable iSPR instrument offers several other key advantages such as reduction in size (14 times), weight (8-11 times), and cost (7-12 times) compared to the conventional (i)SPR instruments mentioned and has potential for future on-site biosensing applications.

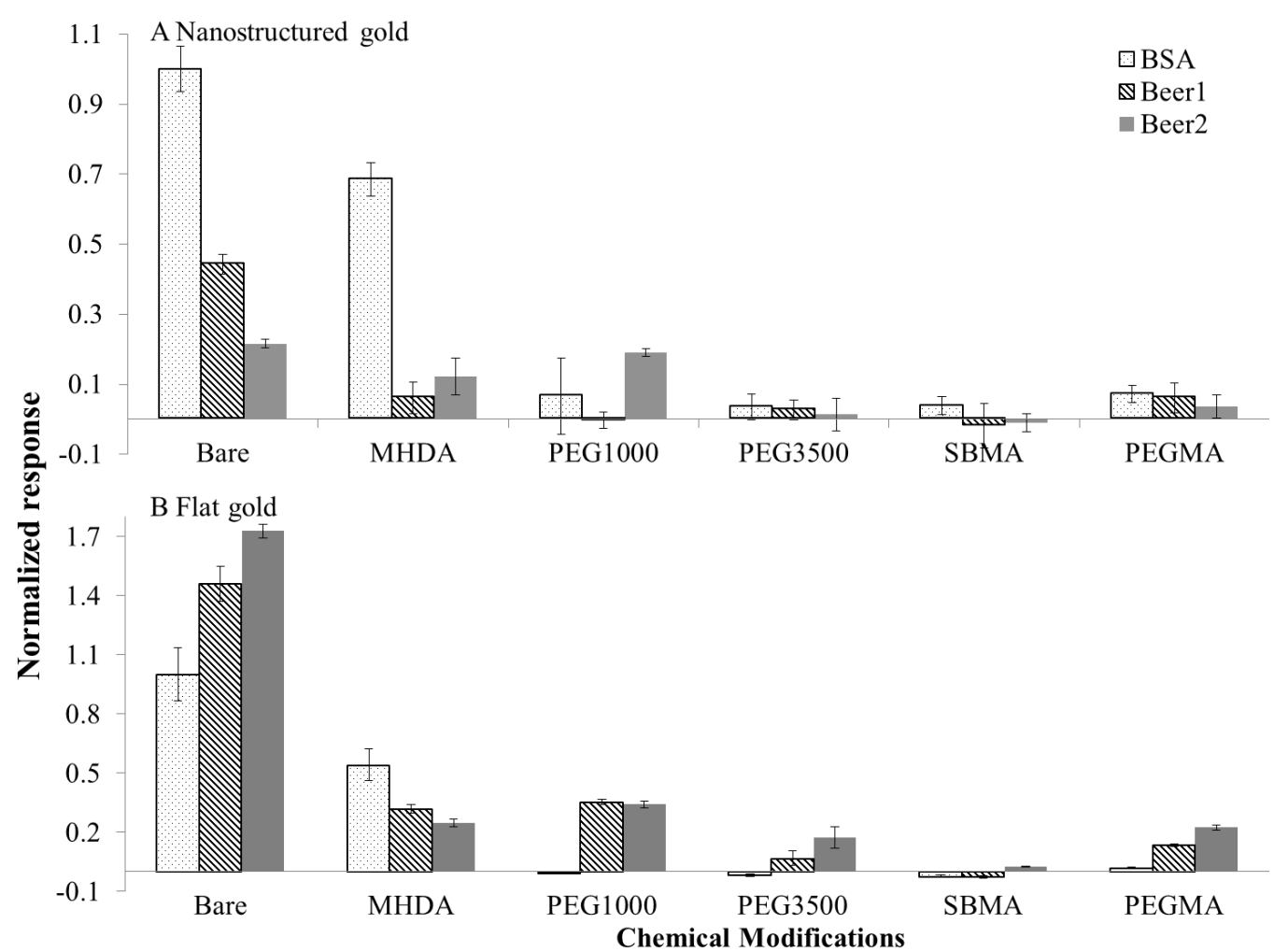

Figure 5. Relative responses measured upon addition of BSA and two different beer samples to unmodified and modified A) nanostructured gold (measured with imaging nanoplasmonics instrument), and B) flat gold (measured in a conventional SPR instrument). The values have been normalized to the response obtained for bare gold upon addition of $1 \mathrm{mg} / \mathrm{mL}$ BSA. The values are an average of three measurements and the errors are represented as error bars (standard deviation of the three measurements). 


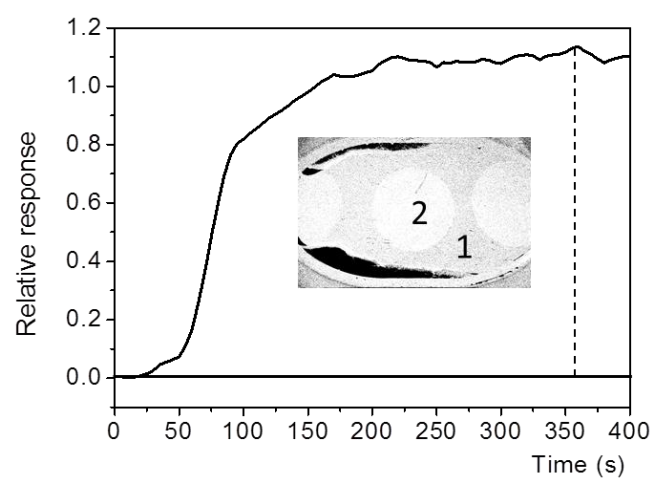

Figure 6. SPR sensorgram generated upon addition of streptavidin $(10 \mu \mathrm{g} / \mathrm{mL}$ diluted in HBS-EP) to a nanostructured gold chip modified with PEG3500 and measured by the portable iSPR instrument. Inset: the chip was locally modified with biotin in region 2 . The sensorgram shown is the response in region 2 after background subtraction of the response recorded in region 1.

\section{Conclusions}

Nanostructured chips were modified with different antifouling chemistries (PEG and zwitterionic polymers) and characterized in detail using AFM, SEM, water contact angle, XPS and DART-HRMS. The antifouling properties of six different surfaces (bare, MHDA, PEG1000, PEG3500, SBMA and PEGMA) were studied using the portable iSPR instrument. Chips modified with PEG3500, SBMA and PEGMA showed the best antifouling properties for the established fouling standard (BSA) as well as a real-life application matrix (beer). Properties (characterization and antifouling) of the iSPR chips and performance of the iSPR instrument were compared with flat gold and conventional SPR (Biacore). Minor differences observed in surface properties without influence on the overall antifouling performance demonstrated transferability of the chemistries. Although the iSPR instrument was nine times less sensitive than the conventional SPR, an approximately ten-fold reduction in size, weight and cost opens up significant possibilities for future atfield applications, such as detection of toxins in beer and barley, allergens in food. ${ }^{7}$

\section{Acknowledgements}

This research received funding from the Netherlands Organisation for Scientific Research (NWO) in the framework of the Technology Area COAST (project nr 053.21.107) with VU Amsterdam, RIKILT, Heineken, Synthon, Technex, EuroProxima, Waterproef as partners and Plasmore and Bionavis as associated partners. We would like to thank Dick J. van Iperen (VU Amsterdam) for constructing the flowcell (Figure S1). 


\section{References}

1. Homola, J., Surface plasmon resonance sensors for detection of chemical and biological species. Chem. Rev. 2008, 108, 462-493.

2. Schasfoort, R. B. M.; Tudos, A. J., Handbook of surface plasmon resonance. The Royal Society of Chemistry: Cambridge, 2008.

3. Rothenhaüsler, B.; Knoll, W., Surface-plasmon microscopy. Nature 1988, 332, 615-617.

4. Yeatman, E.; Ash, E. A., Surface plasmon microscopy. Electron. Lett. 1987, 23, 1091-1092.

5. Scarano, S.; Mascini, M.; Turner, A. P. F.; Minunni, M., Surface plasmon resonance imaging for affinity-based biosensors. Biosens. Bioelectron. 2010, 25, 957-966.

6. Dorokhin, D.; Haasnoot, W.; Franssen, M. C. R.; Zuilhof, H.; Nielen, M. W. F., Imaging surface plasmon resonance for multiplex microassay sensing of mycotoxins. Anal. Bioanal. Chem. 2011, 400, 3005-3011.

7. Raz, S. R.; Liu, H.; Norde, W.; Bremer, M. G. E. G., Food allergens profiling with an imaging surface plasmon resonance-based biosensor. Anal. Chem. 2010, 82, 8485-8491.

8. Beusink, J. B.; Lokate, A. M. C.; Besselink, G. A. J.; Pruijn, G. J. M.; Schasfoort, R. B. M., Angle-scanning SPR imaging for detection of biomolecular interactions on microarrays. Biosens. Bioelectron. 2008, 23, 839-844.

9. Valsesia, A.; Colpo, P.; Rossi, F.; Marabelli, F., A surface plasmon resonance sensing method and sensing system. European Patent 2010, EP 2264438 A1.

10. Bottazzi, B.; Fornasari, L.; Frangolho, A.; Giudicatti, S.; Mantovani, A.; Marabelli, F.; Marchesini, G.; Pellacani, P.; Therisod, R.; Valsesia, A., Multiplexed label-free optical biosensor for medical diagnostics. J. Biomed. Opt. 2014, 19, 017006-017010.

11. Valsesia, A.; Marabelli, F.; Giudicatti, S.; Marchesini, G. R.; Rossi, F.; Colpo, P., SPR sensor device with nanostructure. World Patent 2013, WO 2013/007448 A1.

12. Breault-Turcot, J.; Masson, J.-F., Nanostructured substrates for portable and miniature SPR biosensors. Anal. Bioanal. Chem. 2012, 403, 1477-1484.

13. Najiminaini, M.; Ertorer, E.; Kaminska, B.; Mittler, S.; Carson, J. J. L., Surface plasmon resonance sensing properties of a 3D nanostructure consisting of aligned nanohole and nanocone arrays. Analyst 2014, 139, 1876-1882.

14. Alleyne, C. J.; Kirk, A. G.; McPhedran, R. C.; Nicorovici, N.-A. P.; Maystre, D., Enhanced SPR sensitivity using periodic metallic structures. Opt. Express 2007, 15, 8163-8169.

15. Fan, M.; Andrade, G. F. S.; Brolo, A. G., A review on the fabrication of substrates for surface enhanced raman spectroscopy and their applications in analytical chemistry. Anal. Chim. Acta 2011, 693, 7-25.

16. Bolduc, O. R.; Masson, J.-F., Advances in surface plasmon resonance sensing with nanoparticles and thin films: Nanomaterials, surface chemistry, and hybrid plasmonic techniques. Anal. Chem. 2011, 83, 8057-8062.

17. Anker, J. N.; Hall, W. P.; Lyandres, O.; Shah, N. C.; Zhao, J.; Van Duyne, R. P., Biosensing with plasmonic nanosensors. Nat. Mater. 2008, 7, 442-453.

18. Ladd, J.; Zhang, Z.; Chen, S.; Hower, J. C.; Jiang, S., Zwitterionic polymers exhibiting high resistance to nonspecific protein adsorption from human serum and plasma. Biomacromolecules 2008, 9, 1357-1361.

19. Zhang, Z.; Chen, S.; Chang, Y.; Jiang, S., Surface grafted sulfobetaine polymers via atom transfer radical polymerization as superlow fouling coatings. J. Phys. Chem. B 2006, 110, 10799-10804.

20. Uchida, K.; Otsuka, H.; Kaneko, M.; Kataoka, K.; Nagasaki, Y., A reactive poly(ethylene glycol) layer to achieve specific surface plasmon resonance sensing with a high $\mathrm{S} / \mathrm{N}$ ratio: The substantial role of a short underbrushed PEG layer in minimizing nonspecific adsorption. Anal. Chem. 2005, 77, 1075-1080.

21. Chang, Y.; Liao, S.-C.; Higuchi, A.; Ruaan, R.-C.; Chu, C.-W.; Chen, W.-Y., A highly stable nonbiofouling surface with well-packed grafted zwitterionic polysulfobetaine for plasma protein repulsion. Langmuir 2008, 24, 5453-5458.

22. Mi, L.; Jiang, S., Integrated antimicrobial and nonfouling zwitterionic polymers. Angew. Chem., Int. Ed. 2014, 53, 1746-1754.

23. Löfås, S.; Johnsson, B., A novel hydrogel matrix on gold surfaces in surface plasmon resonance sensors for fast and efficient covalent immobilization of ligands. J. Chem. Soc., Chem. Commun. 1990, 1526-1528.

24. Leckband, D.; Sheth, S.; Halperin, A., Grafted poly(ethylene oxide) brushes as nonfouling surface coatings. J. Biomater. Sci., Polym. Ed. 1999, 10, 1125-1147.

25. Chen, S.; Li, L.; Zhao, C.; Zheng, J., Surface hydration: Principles and applications toward low-fouling/nonfouling biomaterials. Polymer 2010, 51, 5283-5293. 
26. Unsworth, L. D.; Sheardown, H.; Brash, J. L., Protein-resistant poly(ethylene oxide)-grafted surfaces: Chain density-dependent multiple mechanisms of action. Langmuir 2008, 24, 1924-1929.

27. Rodriguez-Emmenegger, C.; Brynda, E.; Riedel, T.; Sedlakova, Z.; Houska, M.; Alles, A. B., Interaction of blood plasma with antifouling surfaces. Langmuir 2009, 25, 6328-6333.

28. Lee, B. S.; Chi, Y. S.; Lee, K.-B.; Kim, Y.-G.; Choi, I. S., Functionalization of poly(oligo(ethylene glycol) methacrylate) films on gold and $\mathrm{Si} / \mathrm{SiO}_{2}$ for immobilization of proteins and cells: SPR and QCM studies. Biomacromolecules 2007, 8, 3922-3929.

29. Schlenoff, J. B., Zwitteration: coating surfaces with zwitterionic functionality to reduce nonspecific adsorption. Langmuir 2014, 30, 9625-9636.

30. Estephan, Z. G.; Schlenoff, P. S.; Schlenoff, J. B., Zwitteration as an alternative to PEGylation. Langmuir 2011, 27, 6794-6800.

31. Giudicatti, S.; Valsesia, A.; Marabelli, F.; Colpo, P.; Rossi, F., Plasmonic resonances in nanostructured gold/polymer surfaces by colloidal lithography. Phys. Status Solidi A 2010, 207, 935-942.

32. Lahiri, J.; Ostuni, E.; Whitesides, G. M., Patterning ligands on reactive SAMs by microcontact printing. Langmuir 1999, 15, 2055-2060.

33. Nguyen, A. T.; Baggerman, J.; Paulusse, J. M. J.; Zuilhof, H.; van Rijn, C. J. M., Bioconjugation of protein-repellent zwitterionic polymer brushes grafted from silicon nitride. Langmuir 2012, 28, 604-610.

34. Hofmann, S., Quantitative analysis (data evaluation), in auger- and x-ray photoelectron spectroscopy in materials science. In Springer-Verlag: Berlin Heidelberg, 2013.

35. Petrovykh, D. Y.; Kimura-Suda, H.; Tarlov, M. J.; Whitman, L. J., Quantitative characterization of DNA Films by X-ray photoelectron spectroscopy. Langmuir 2004, 20, 429440.

36. Manova, R. K.; Claassen, F. W.; Nielen, M. W. F.; Zuilhof, H.; van Beek, T. A., Ambient mass spectrometry of covalently bound organic monolayers. Chem. Comm. 2013, 49, 922-924.

37. IRiedel, T.; Riedelová-Reicheltová, Z.; Májek, P.; Rodriguez-Emmenegger, C.; Houska, M.; Dyr, J. E.; Brynda, E., Complete identification of proteins responsible for human blood plasma fouling on poly(ethylene glycol)-based surfaces. Langmuir 2013, 29, 3388-3397.

38. Hosseini, S.; Ibrahim, F.; Djordjevic, I.; Koole, L. H., Recent advances in surface functionalization techniques on polymethacrylate materials for optical biosensor applications. Analyst 2014, 139, 2933-2943.

39. Jones, D. M.; Brown, A. A.; Huck, W. T. S., Surface-initiated polymerizations in aqueous media: effect of initiator density. Langmuir 2002, 18, 1265-1269.

40. Lu, H. B.; Campbell, C. T.; Castner, D. G., Attachment of functionalized poly(ethylene glycol) films to gold surfaces. Langmuir 2000, 16, 1711-1718.

41. Nguyen, A. T.; Baggerman, J.; Paulusse, J. M. J.; van Rijn, C. J. M.; Zuilhof, H., Stable protein-repellent zwitterionic polymer brushes grafted from silicon nitride. Langmuir 2011, 27, 2587-2594.

42. Manova, R. K.; Joshi, S.; Debrassi, A.; Bhairamadgi, N. S.; Roeven, E.; Gagnon, J.; Tahir, M. N.; Claassen, F. W.; Scheres, L. M. W.; Wennekes, T.; Schroën, K.; van Beek, T. A.; Zuilhof, H.; Nielen, M. W. F., Ambient surface analysis of organic monolayers using direct analysis in real time orbitrap mass spectrometry. Anal. Chem. 2014, 86, 2403-2411.

43. Kpegba, K.; Spadaro, T.; Cody, R. B.; Nesnas, N.; Olson, J. A., Analysis of self-assembled monolayers on gold surfaces using direct analysis in real time mass spectrometry. Anal. Chem. 2007, 79, 5479-5483.

44. Wong, N. P.; Jenness, R.; Keeney, M.; Marth, E. H., Fundamentals of dairy chemistry. Springer US New York, 1988. 


\section{Supporting information}

A

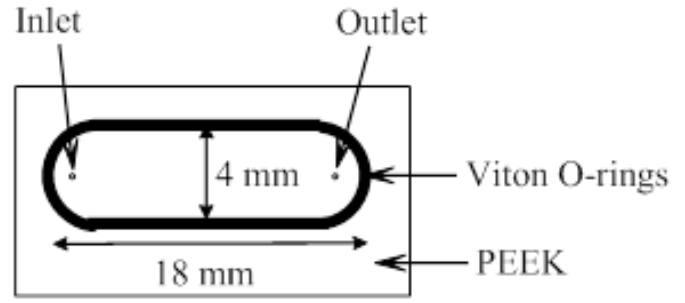

B

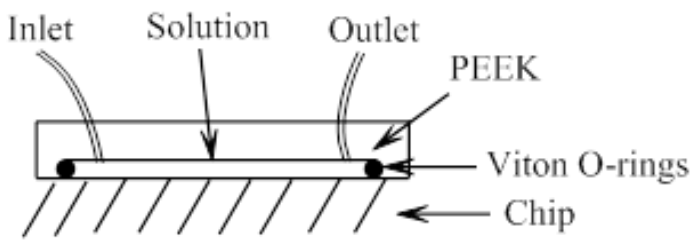

Figure S1. Schematic representation of the A) top and B) side view of the PEEK flow-cell, with Viton O-rings, used for partial modification of nanostructured iSPR chips. The flow-cell has an inlet that can be connected to a syringe pump to maintain a constant flow of solution.
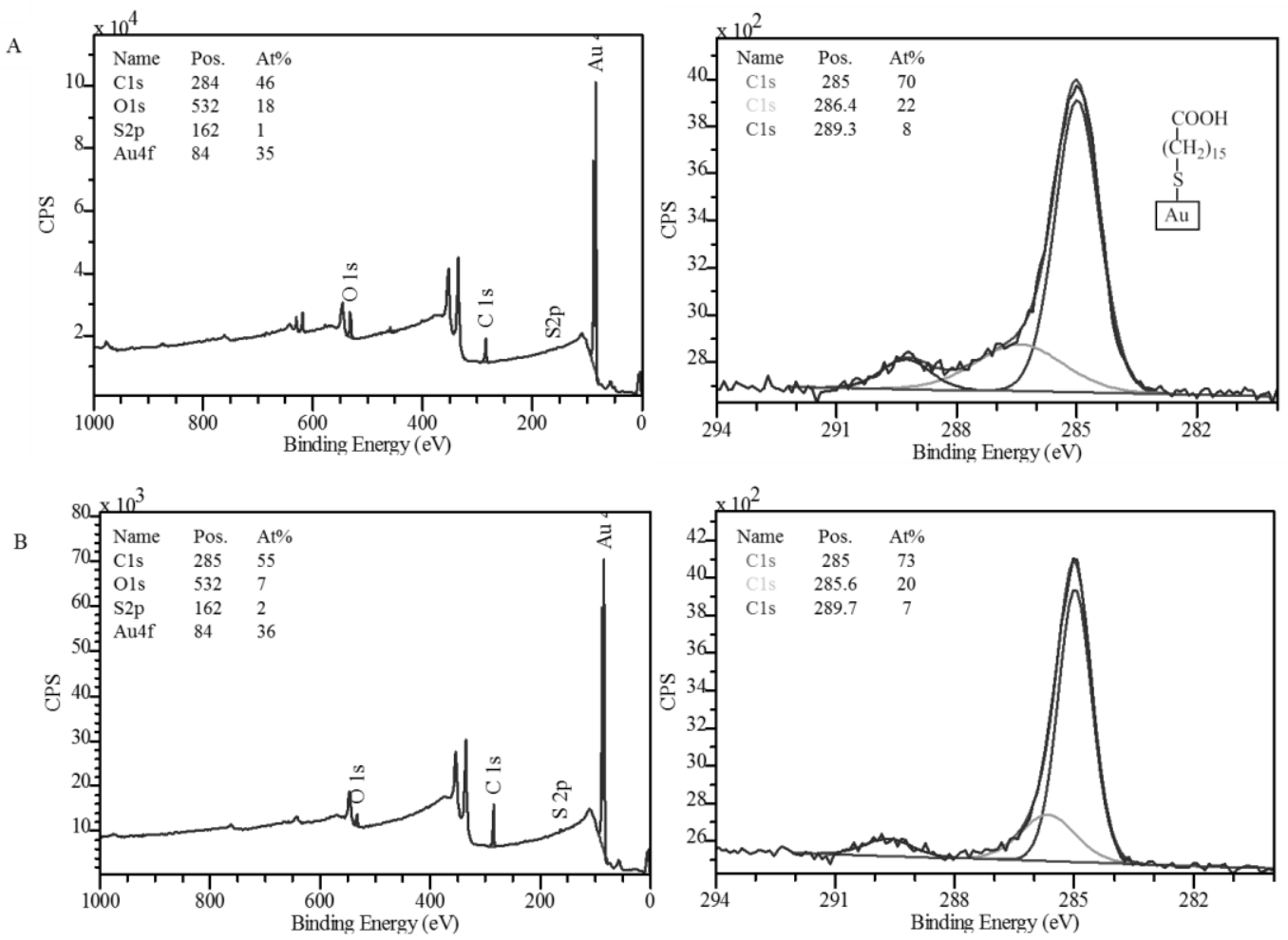

Figure S2. XPS survey (left) and C1s narrow scan (right) spectra of MHDA modified a) nanostructured and b) flat gold. 

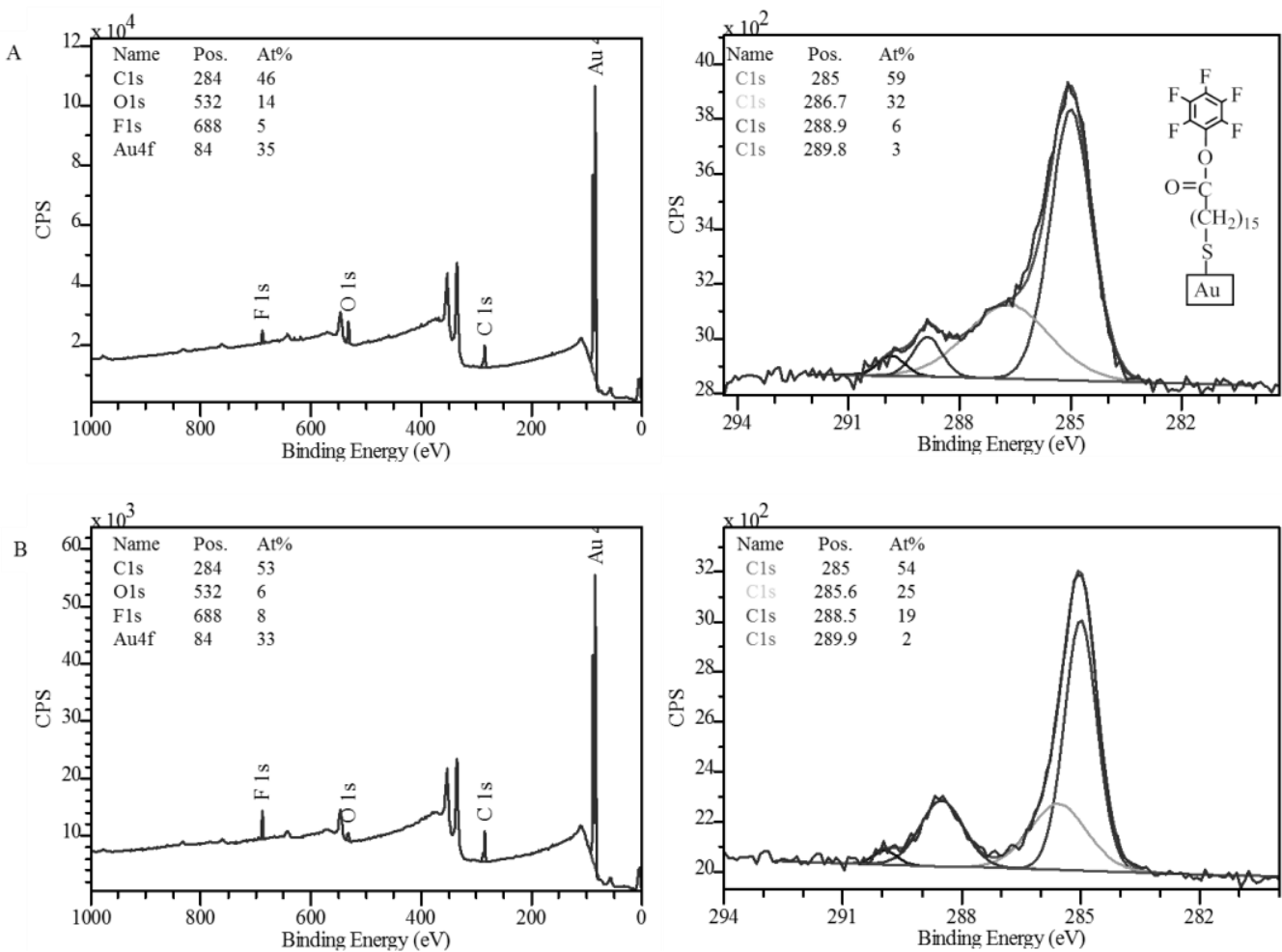

Figure S3. XPS survey (left) and C1s narrow scan (right) spectra of PFP modified A) nanostructured and B) flat gold.
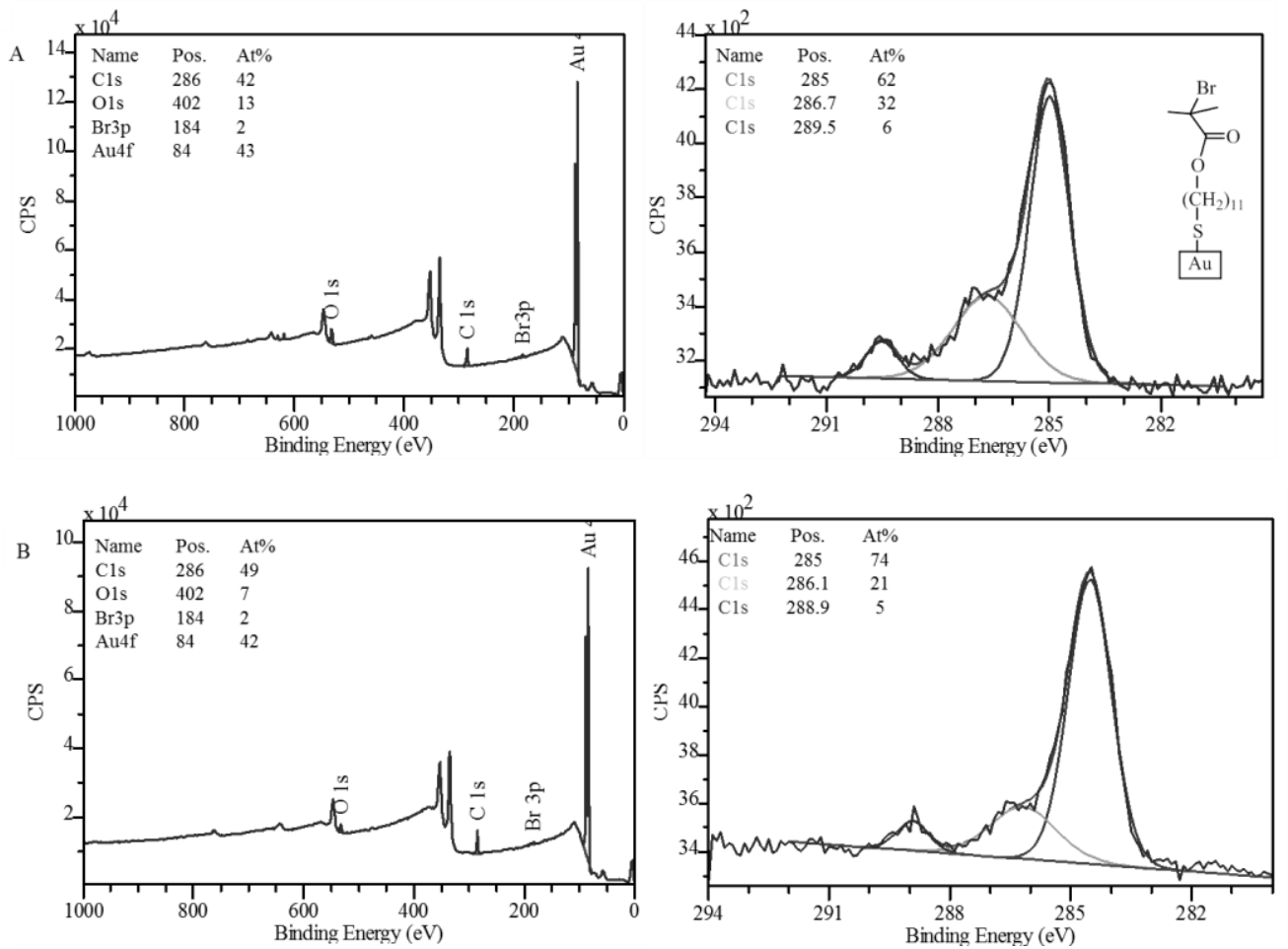

Figure S4. XPS survey (left) and C1s narrow scan (right) spectra of Izo-Br modified A) nanostructured and B) flat gold. 

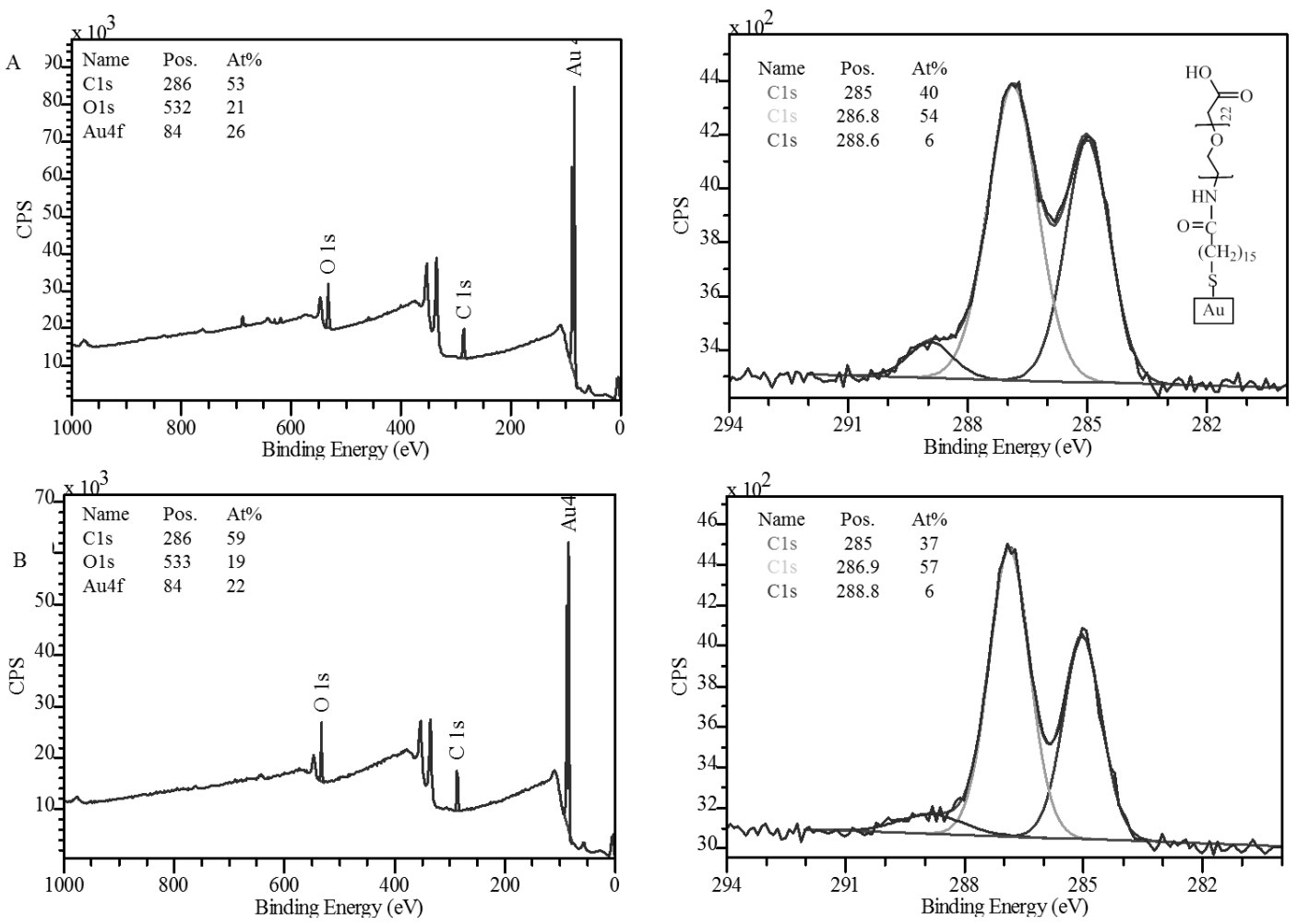

Figure S5. XPS survey (left) and C1s narrow scan (right) spectra of PEG1000 modified A) nanostructured and B) flat gold.
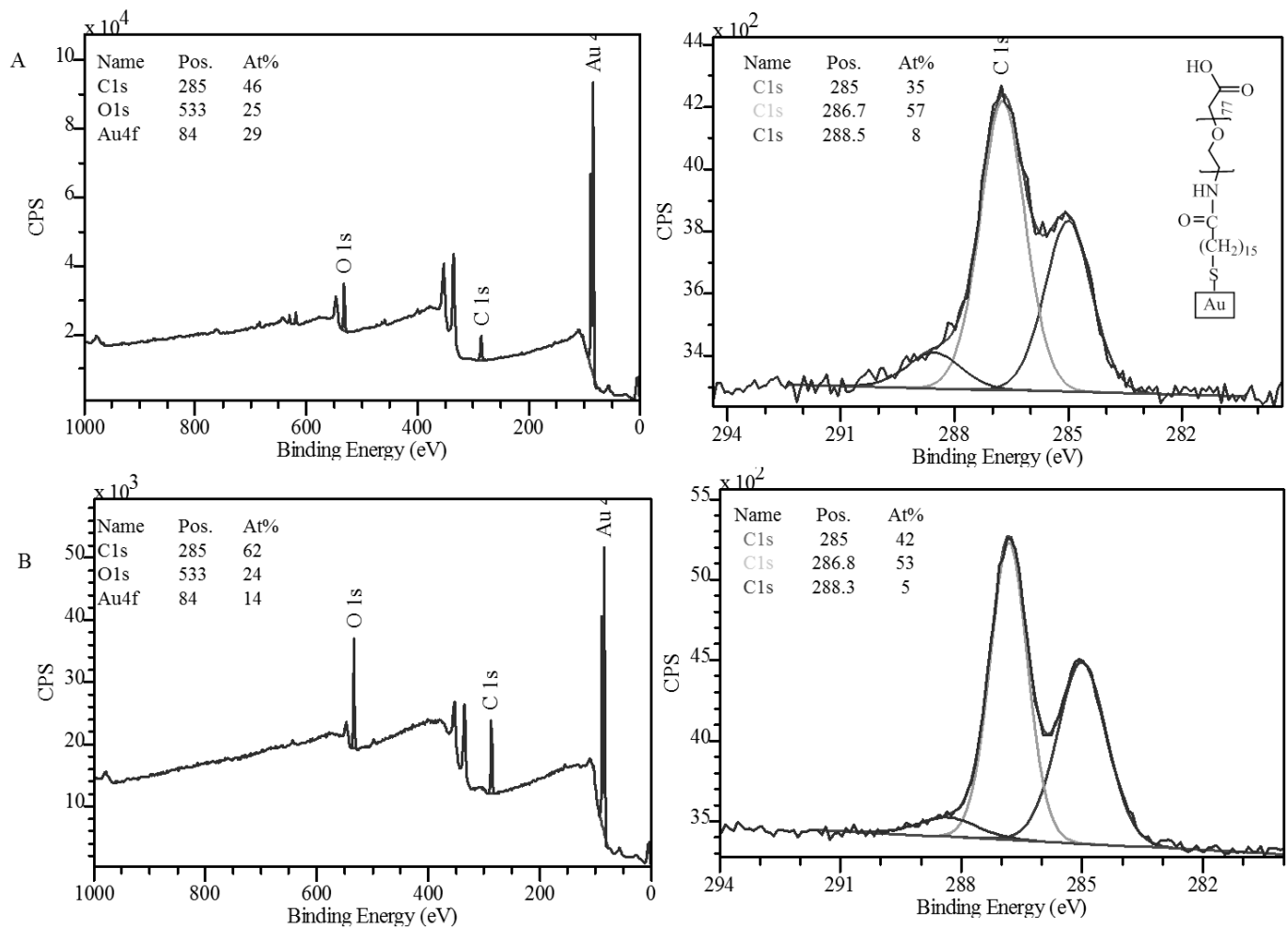

Figure S6. XPS survey (left) and C1s narrow scan (right) spectra of PEG3500 modified A) nanostructured and B) flat gold. 

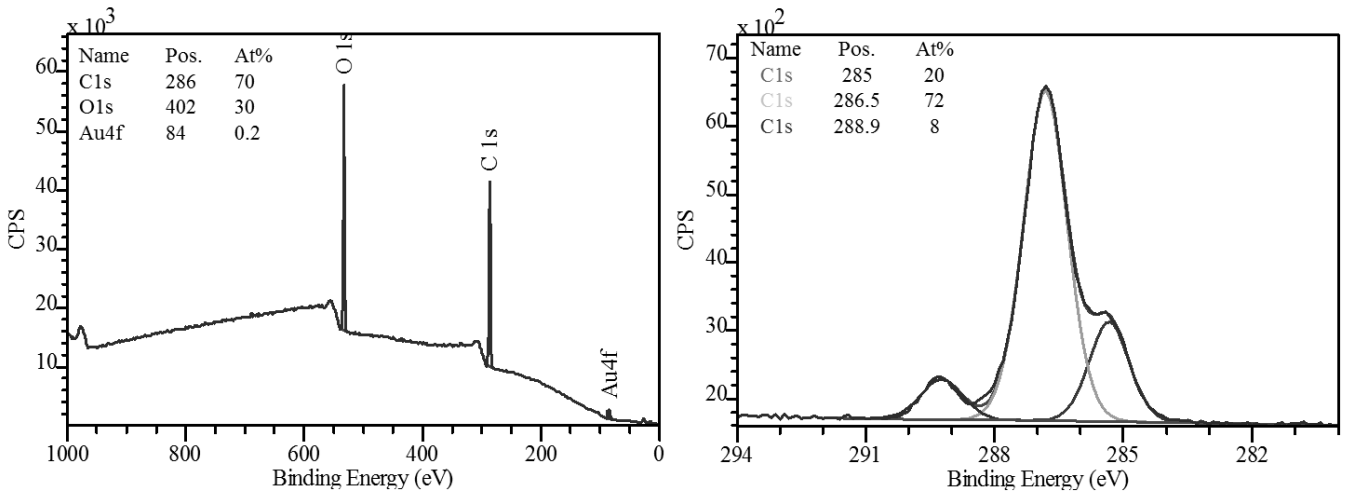

Figure S7. XPS survey (left) and C1s narrow scan (right) spectra of PEGMA modified flat gold.
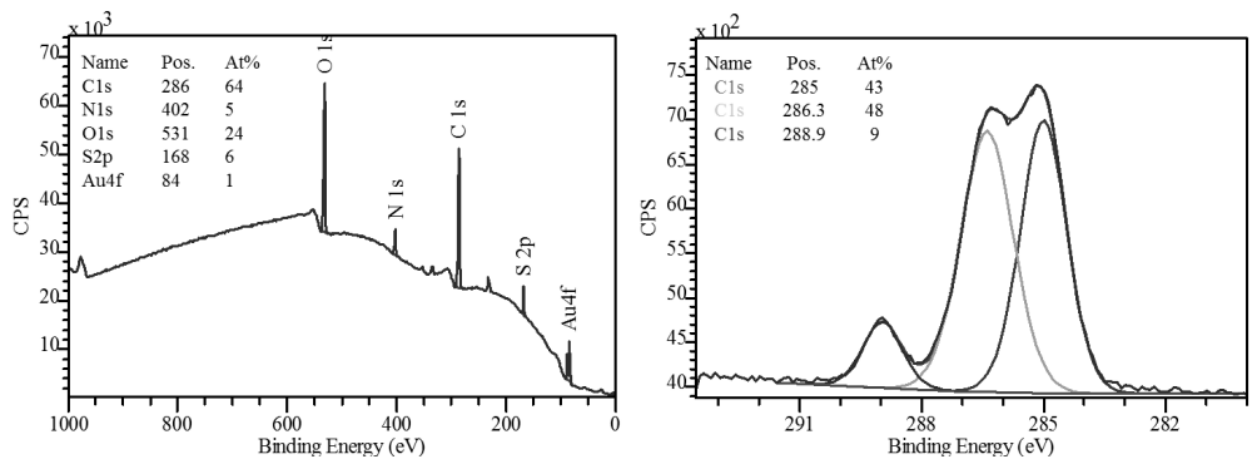

Figure S8. XPS survey (left) and C1s narrow scan (right) spectra of SBMA modified flat gold. 

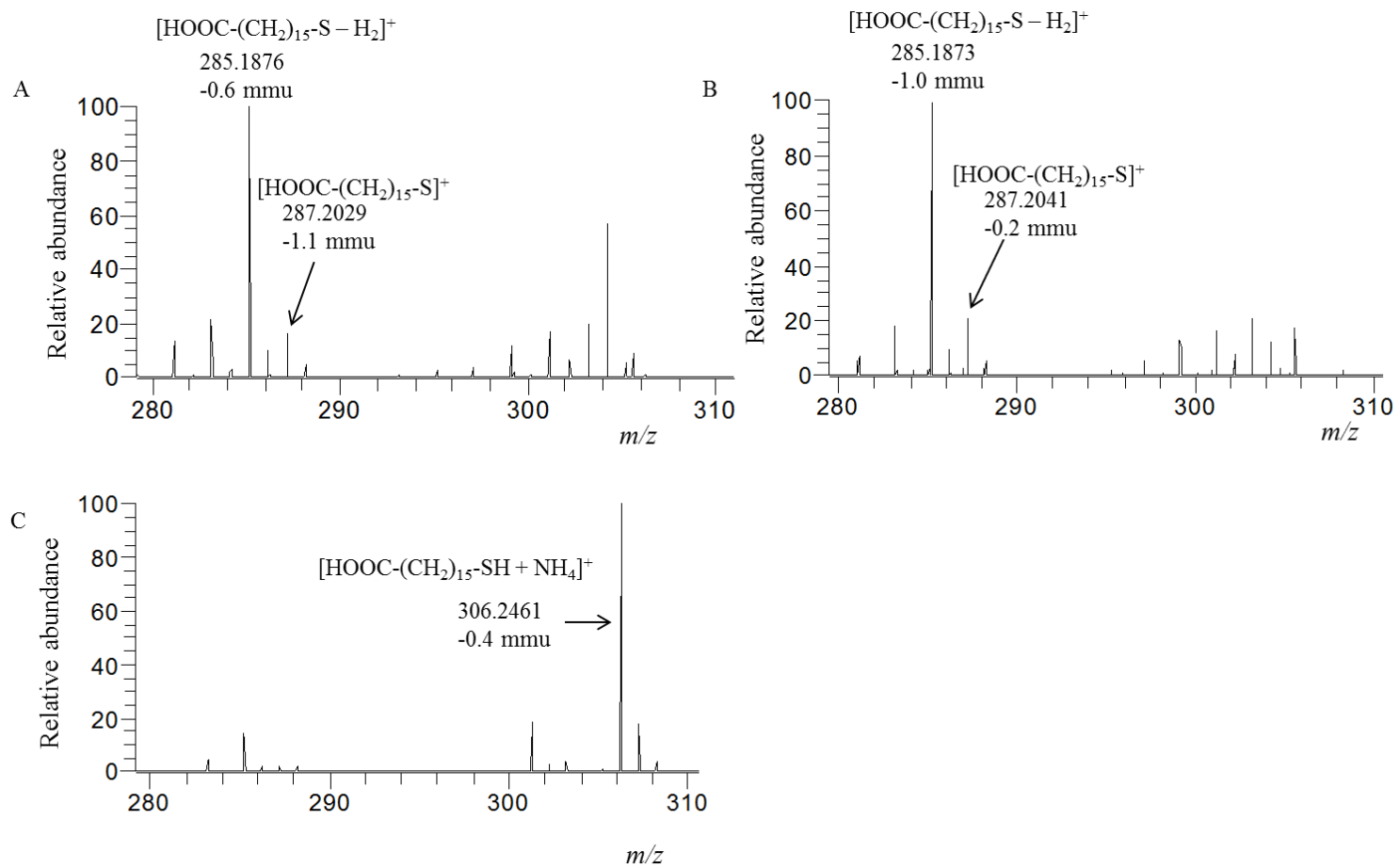

Figure 59. DART-HRMS of MHDA A) modified nanostructured gold, B) modified flat gold, and C) solution placed on glass, measured in positive mode.
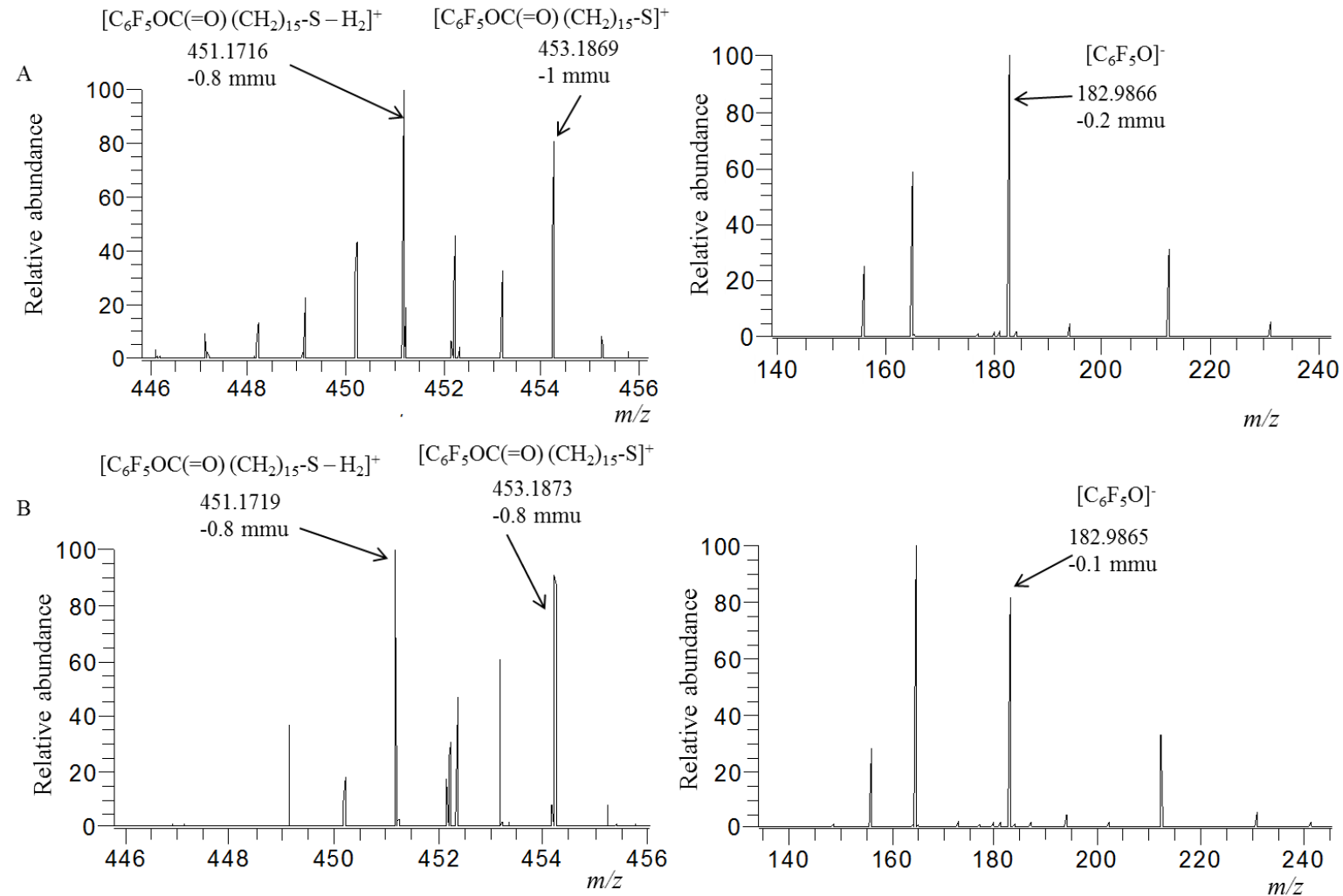

Figure S10. DART-HRMS of PFP modified A) nanostructured and B) flat gold measured in positive (left) and negative (right) mode. 

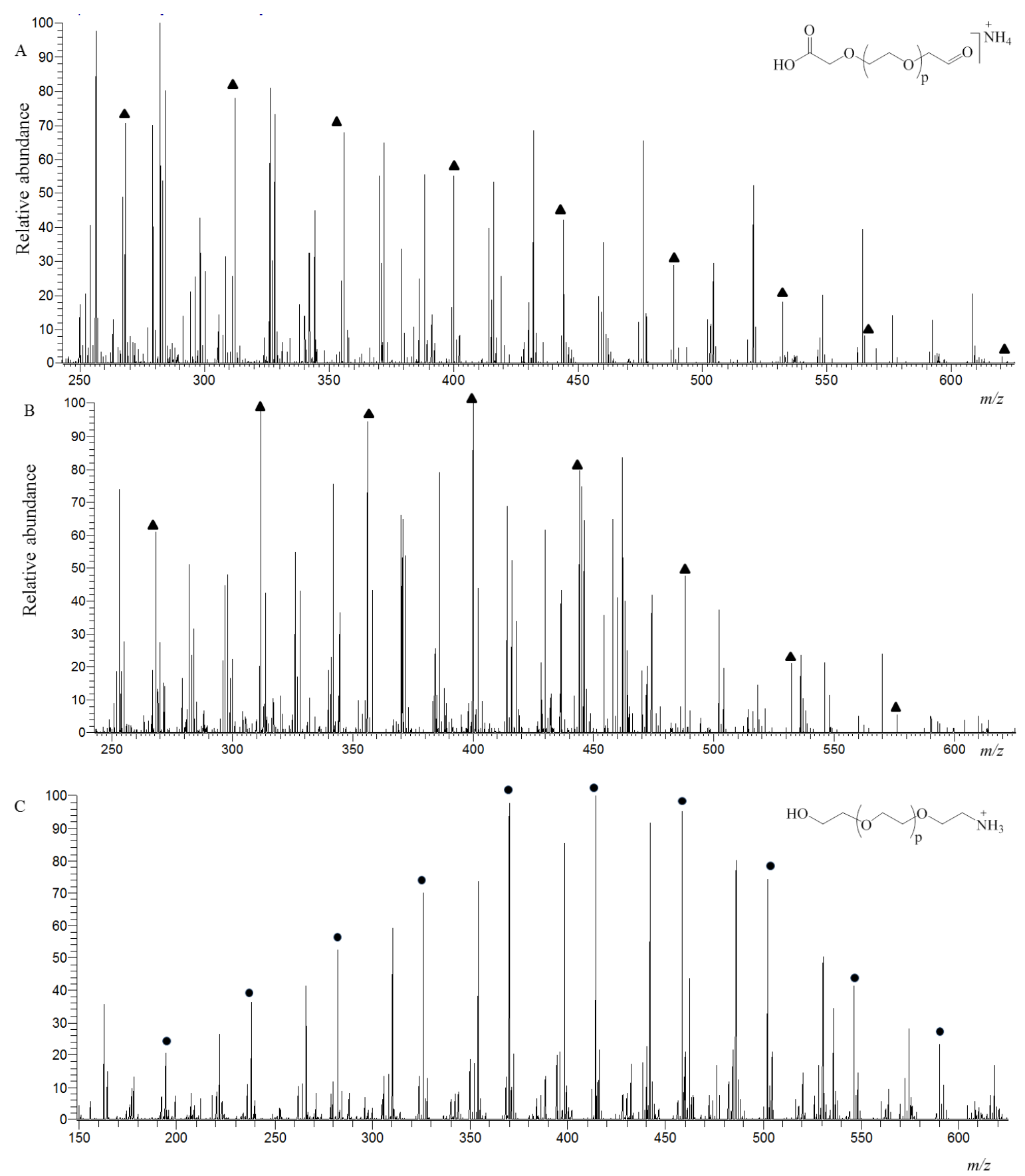

Figure S11. DART-HRMS of PEG1000 A) modified nanostructured gold, B) modified flat gold, and C) solution placed on glass, measured in positive mode. A representative ion series is indicated in the graph. 


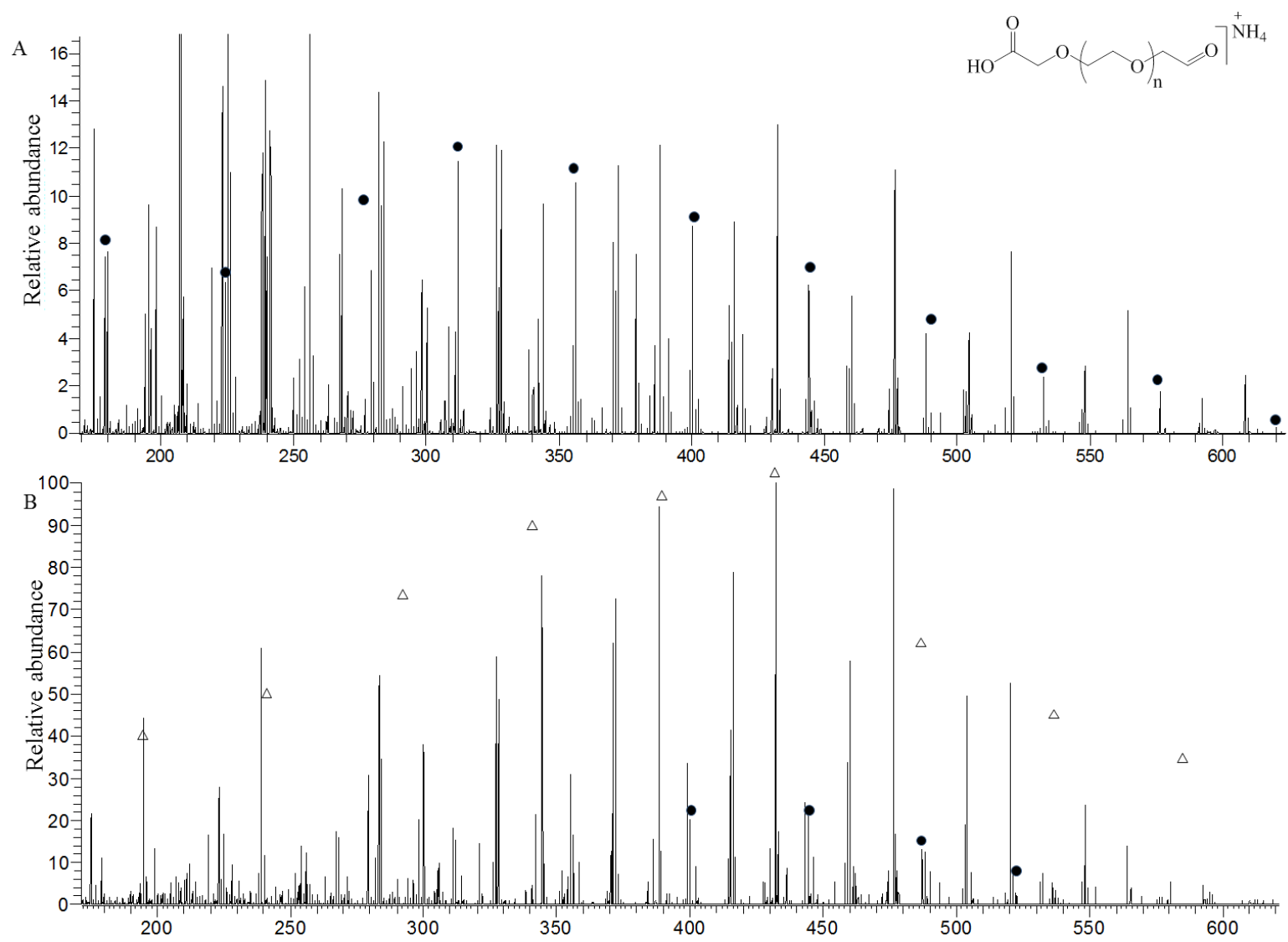

Figure S12. DART-HRMS of PEG3500 modified A) nanostructured and B) flat gold measured in positive mode. A representative ion series is indicated in the graph.
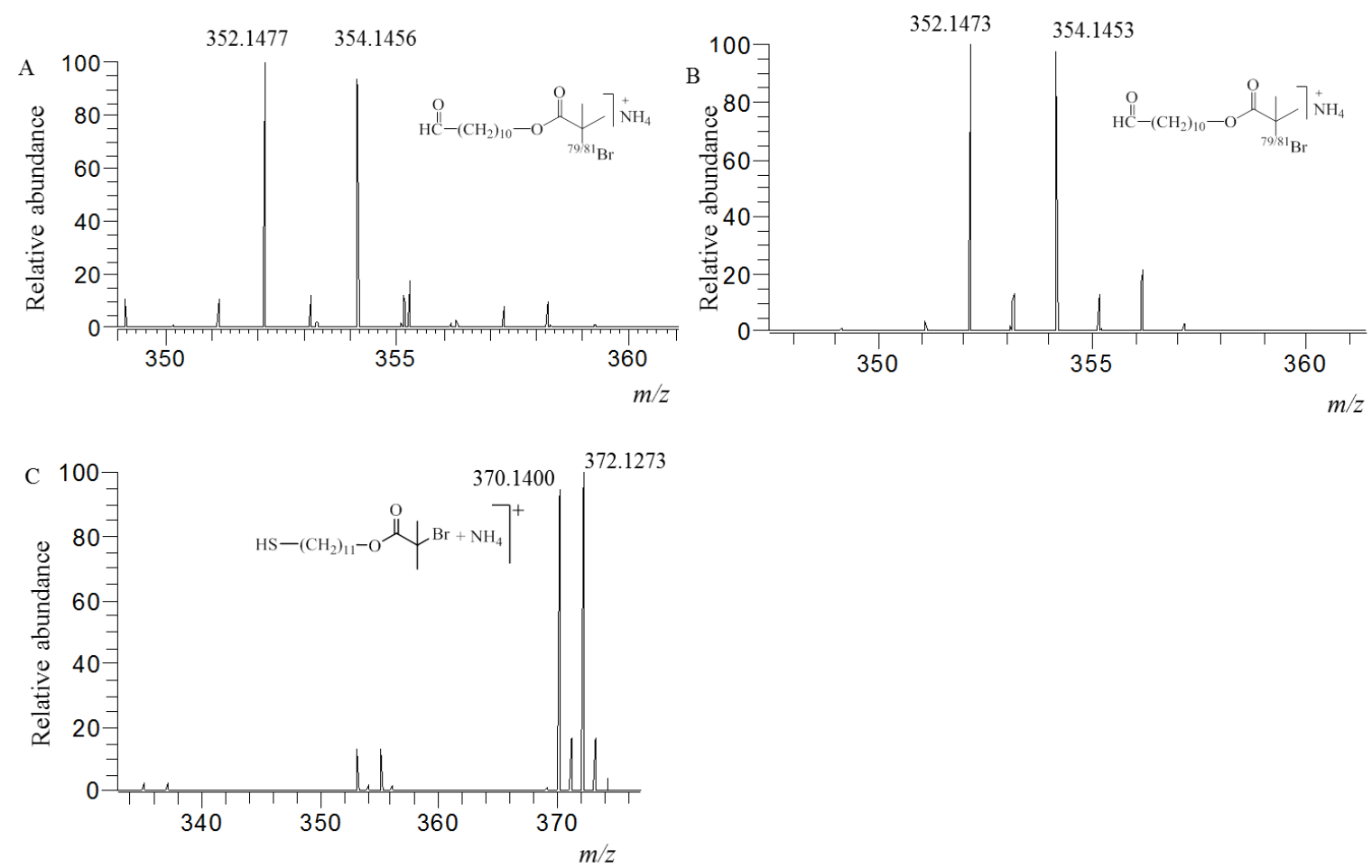

Figure S13. DART-HRMS of Izo-Br A) modified nanostructured gold, B) modified flat gold and, C) solution placed on glass, measured in positive mode. 

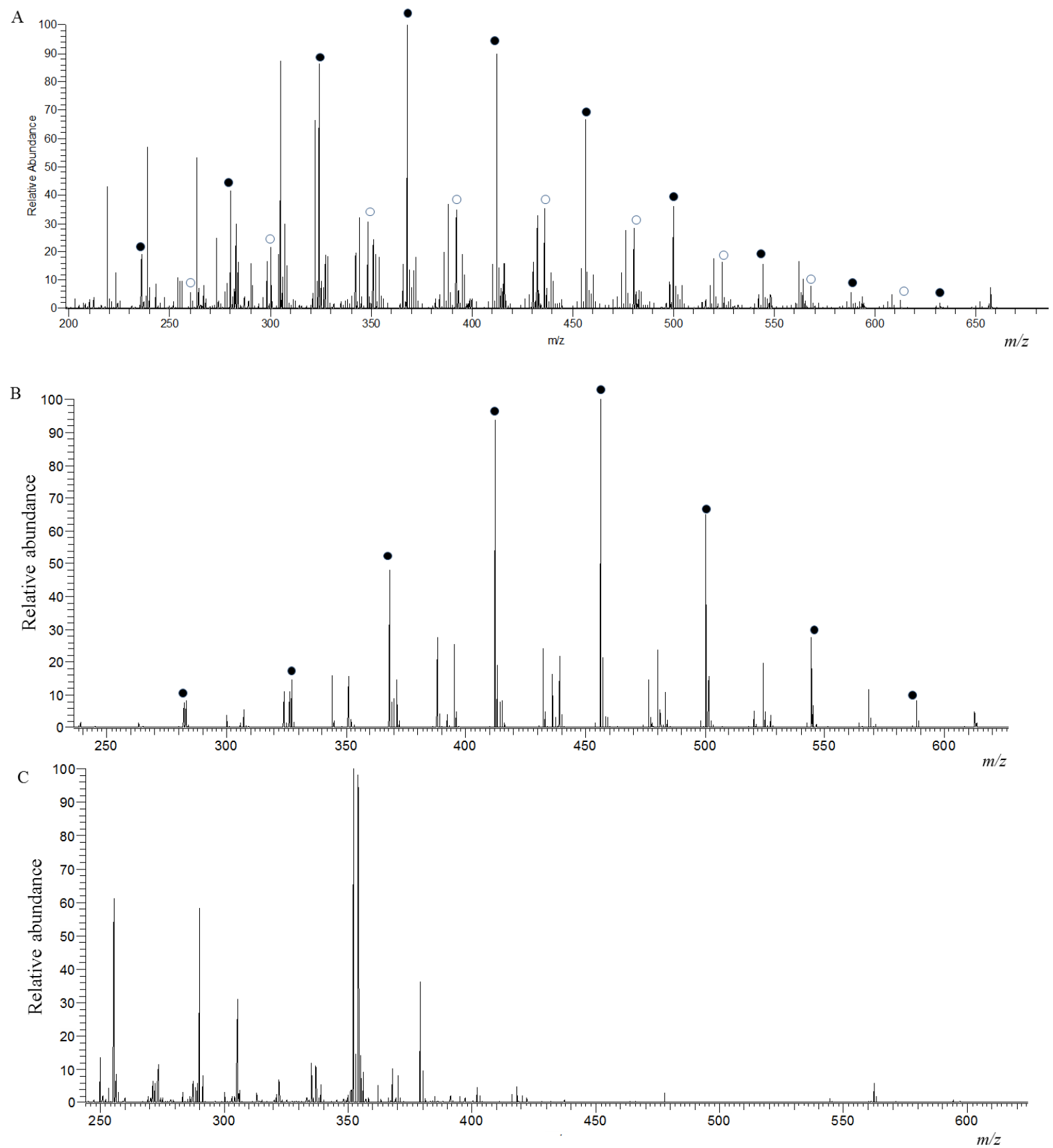

Figure S14. DART-HRMS of A) PEGMA modified flat gold, B) PEGMA monomer on Izo-Br modified nanostructure gold before, and $C$ ) after washing with water and methanol and sonicating in methanol (measured in positive mode). A representative ion series is indicated in the graph. 

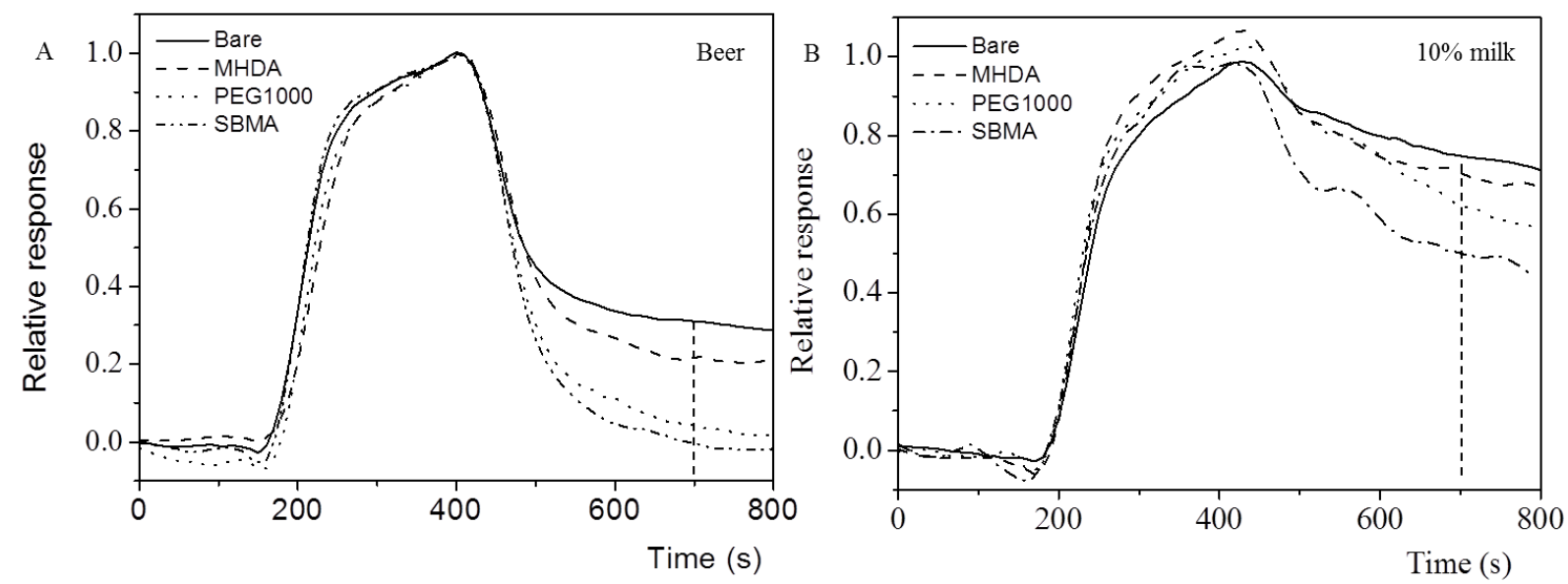

Figure S15. SPR sensorgrams generated by the iSPR instrument upon addition of A) beer 1 and $B$ ) $10 \%$ milk to bare and differently modified nanostructured gold. The vertical dashed line indicates the time point where the relative response was measured to obtain amount of fouling.

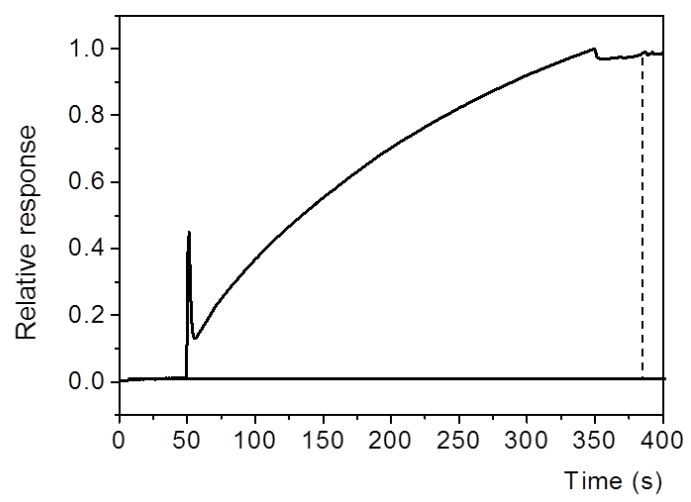

Figure S16. SPR sensorgram generated upon addition of streptavidin $(10 \mu \mathrm{g} / \mathrm{mL}$ diluted in HBSEP) to flat gold with PEG3500 chip further modified with biotin using Biacore 3000 .

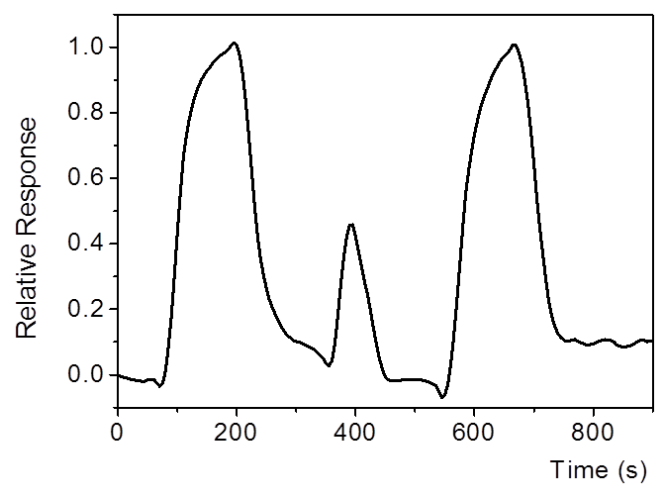

Figure S17. SPR sensorgram generated by the iSPR instrument upon two successive cycles of addition of beer 1 to PEG3500 modified nanostructured gold. The chip was regenerated in between the two cycles $(t=350-450 \mathrm{~s})$ using $10 \mathrm{mM} \mathrm{NaOH}$. 



\section{Chapter 3}

\section{Multiplex surface plasmon resonance biosensing and its transferability towards imaging nanoplasmonics for detection of mycotoxins in barley}

Sweccha Joshi, ${ }^{1,2}$, Anna Segarra-Fas ${ }^{1}$, J. Peters ${ }^{3}$, Han Zuilhof ${ }^{1,4}$, Teris A. van Beek ${ }^{1}$, Michel W.F. Nielen ${ }^{1,3}$

${ }^{1}$ Laboratory of Organic Chemistry, Wageningen University, Dreijenplein 8, 6703 HB Wageningen, The Netherlands

${ }^{2}$ TI-COAST, Science Park 904, 1098 XH, Amsterdam, The Netherlands ${ }^{3}$ RIKILT Wageningen UR, P.O. Box 230, 6700 AE Wageningen, The Netherlands

${ }^{4}$ Department of Chemical and Materials Engineering, King Abdulaziz University, Jeddah, Saudi Arabia

This chapter has been published in:

Analyst, 2016, 141, 1307-1318.

Flat SPR chip

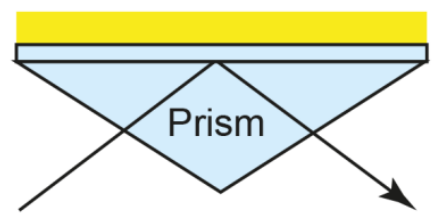

LED

Detector
Nanostructured iSPR chip

Side view

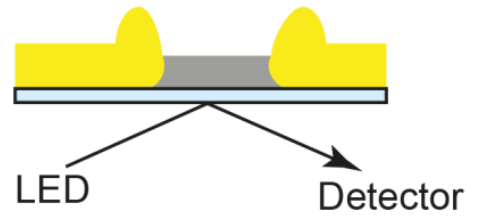

Imaging nanoplasmonics, no prism

6-plex mycotoxin assay

Single chip: Detection of DON, ZEA, T-2, OTA, FB ${ }_{1}$, AFB $_{1}$
Top view

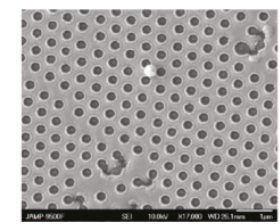

Chip 1: Detection of DON, ZEA, T-2

Chip 2: Detection of OTA, FB, AFB $_{1}$
Benchtop SPR with Prism

Double 3-plex mycotoxin assay 1 


\section{Abstract}

A 6-plex competitive inhibition immunoassay for mycotoxins in barley was developed on a prototype portable nanostructured imaging surface plasmon resonance (iSPR) instrument, also referred to as imaging nanoplasmonics. As a benchmark for the prototype nanoplasmonics instrument, first a double 3-plex assay was developed for the detection of deoxynivalenol (DON), zearalenone (ZEA), T-2 toxin (T-2), ochratoxin A (OTA), fumonisin $B_{1}\left(F B_{1}\right)$ and aflatoxin $B_{1}\left(A F B_{1}\right)$ using a well-established benchtop SPR instrument and two biosensor chips. To this end, ovalbumin (OVA) conjugates of mycotoxins were immobilized on the chip via amine coupling. The SPR response was then recorded upon injection of a mixture of antibodies at a fixed concentration and the sample (or matrix-matched standard) over a chip with immobilized mycotoxin-OVA conjugates. The chips were regenerated after each sample using $10 \mathrm{mM} \mathrm{HCl}$ and $20 \mathrm{mM}$ $\mathrm{NaOH}$ and could be used for at least 60 cycles. The limits of detection in barley (in $\mu \mathrm{g} / \mathrm{kg}$ ) were determined to be 26 for DON, 6 for ZEA, 0.6 for T-2, 3 for OTA, 2 for $\mathrm{FB}_{1}$ and 0.6 for $\mathrm{AFB}_{1}$. Preliminary in-house validation showed that $\mathrm{DON}, \mathrm{T}-2, \mathrm{ZEA}$ and $\mathrm{FB}_{1}$ can be detected at the European Union regulatory limits, while for OTA and AFB $_{1}$ sensitivities should be improved. Furthermore, measurement of naturally contaminated barley showed that the assay can be used as a semi-quantitative screening method for mycotoxins prior to liquid chromatography tandem mass spectrometry (LC-MS/MS). Finally, using the same bio-reagents the assay was transferred to a 6-plex format in the nanoplasmonics instrument and subsequently the two assays were compared. Although less sensitive, the 6-plex portable iSPR assay still allowed detection of DON, T-2, ZEA and $\mathrm{FB}_{1}$ at relevant levels. Therefore, the prototype iSPR shows potential for future applications in rapid in-field and at-line screening of multiple mycotoxins. 


\section{Introduction}

Mycotoxins are secondary metabolites of fungi commonly found in several foods and ingredients such as nuts, cereals (e.g., barley), coffee, oil seeds, fruits and, as a result, also occur in beer, wine as well as in feed. ${ }^{1}$ Barley is used in unprocessed form as well as processed forms in food commodities and is known to be occasionally infected with several fungal species. The most relevant mycotoxins for barley are deoxynivalenol (DON), zearalenone (ZEA or ZEN, referred to as ZEA throughout this article), T-2 and HT-2 toxin, ochratoxin A (OTA), fumonisin $B_{1}\left(F B_{1}\right)$, and aflatoxin $B_{1}\left(A F B_{1}\right){ }^{2}$ Several of these mycotoxins are known for their teratogenicity, mutagenicity and/or carcinogenicity and could be a threat to both human and animal health. According to EU legislation, the maximum levels (MLs) in $\mu \mathrm{g} / \mathrm{kg}$ for unprocessed barley are 1250 for DON, 100 for ZEA, 200 for T-2 (recommendation for sum of T-2 and HT-2), 5 for OTA, 2 for AFB $_{1}$ and 2000 for $\mathrm{FB}_{1}$ (sum of $\mathrm{FB}_{1}$ and $\mathrm{FB}_{2}$ in unprocessed maize). ${ }^{3,4}$

Several methods for mycotoxin detection have been developed in recent years each with their own merits and limitations. ${ }^{5-8}$ Chromatography-based techniques for mycotoxin detection, such as high-pressure liquid chromatography (HPLC) and gas chromatography (GC) in combination with mass spectrometry (MS), are very sensitive and allow quantitative analysis of multiple mycotoxins. ${ }^{9}$ However, they are rather expensive, require skilled personnel, and sometimes involve extensive sample preparation. One or a few mycotoxins have been measured using metal-enhanced fluorescence, ${ }^{10}$ chemiluminescent immunoassay, ${ }^{11,12}$ immuno-polymerase chain reaction, ${ }^{13}$ real-time electrochemical profiling, ${ }^{14}$ fluorescent immunosorbent assay, ${ }^{15}$ microsphere based assay ${ }^{16,17}$ and direct analysis in real time coupled with high-resolution mass spectrometry (DART-HRMS). ${ }^{18}$ Amongst the immunochemical methods, enzymelinked immunosorbent assay (ELISA) and lateral flow immunoassays (LFI) are the most popular approach as they allow, respectively, semi-quantitative or qualitative detection of multiple mycotoxin samples in parallel. ${ }^{19,20}$ Surface plasmon resonance (SPR) based biosensors have emerged as a reliable, label-free and sensitive alternative immunochemical method for the semi-quantitative detection of mycotoxins. ${ }^{21-23}$ SPR, in addition, benefits from real-time monitoring of the interaction kinetics and reusability of the biosensor chip. Within the SPR field, imaging SPR (iSPR) ${ }^{24,25}$ is an interesting development as in principle it allows the simultaneous detection of all mycotoxins of interest using a single sensor chip. The multiplexing capability of iSPR saves both time and cost in terms of bio-reagents as a single injection gives information about multiple mycotoxins. But this potential has not been realized until now as only two mycotoxins have been detected simultaneously using iSPR. ${ }^{26}$ In addition to multiplexing, there is an increasing demand for miniaturized instruments in order to bring the lab to the sample. 
Recently, a new prism-free prototype portable nanostructured iSPR instrument, also referred to as imaging nanoplasmonics (iNPX), was introduced. ${ }^{27}$ However, the merits of this instrument remain to be demonstrated. In a 2015 critical review about nanoplasmonic biosensing ${ }^{28}$ it is observed that no studies in literature perform a fair benchmark test against state-of-the-art SPR. Unfortunately most earlier SPR studies focused on only one or two mycotoxins, ${ }^{29-32}$ while the only report ${ }^{33}$ on multiplex SPR assays neither includes all experimental details nor an application to real sample matrices.

Therefore, the aims of the present study were twofold, the first aim being the development of a double 3-plex benchmark assay (including a reference channel in each chip) for the detection of the six most relevant mycotoxins (DON, ZEA, T-2, OTA, FB ${ }_{1}$ and $\mathrm{AFB}_{1}$ ) in barley using state-of-the-art SPR with flat gold biosensor chips as commonly used in Biacore instruments (Figure $1 \mathrm{~A}$ and B). A detailed study of the cross-reactivity and matrix effects has been performed, together with a preliminary in-house validation and the analysis of naturally contaminated samples. Finally, using the developed benchtop SPR assay as a benchmark, our second aim was to demonstrate the transferability of the state-of-the-art double 3-plex SPR immunoassay to a 6-plex iSPR format with a single nanostructured gold biosensor chip in a portable iSPR instrument (Figure $1 \mathrm{C}$ and $\mathrm{D}$ ) using the same bio-reagents and assay conditions and to compare the nanoplasmonics iSPR assay with the benchmark SPR assay.
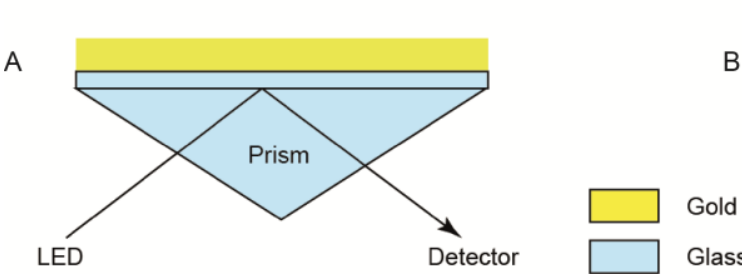

Glass PMMA

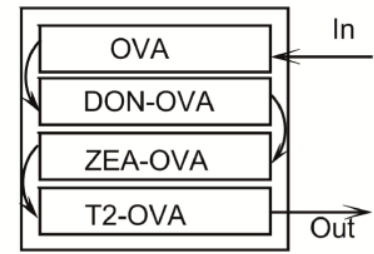

Chip 1

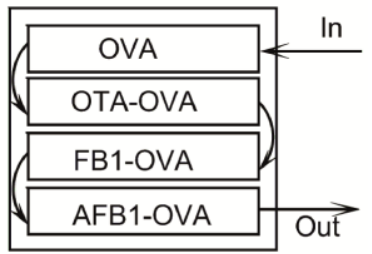

Chip 2

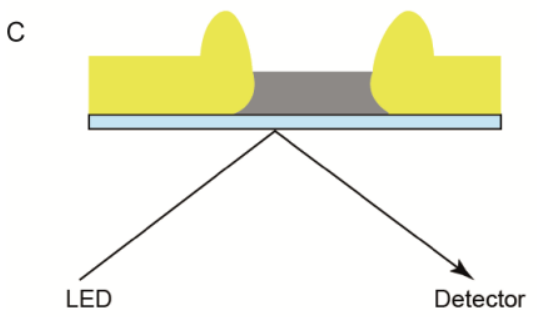

D

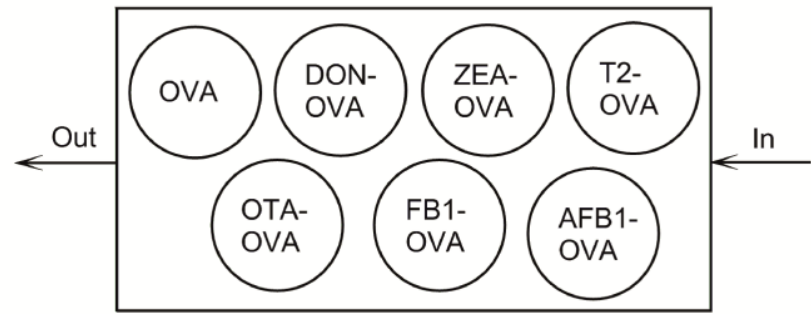

Figure 1. Schematic representation of $A$ ) a benchtop state-of-the-art SPR setup with flat gold as sensor surface and a prism to couple the light into the gold film B) chip microfluidic array for double 3-plex assay (two chips each with an OVA channel as reference and three toxin conjugates) C) portable imaging SPR (iSPR) setup with nanostructured gold as sensor surface thus omitting the need for any prism and D) chip microfluidic array for 6-plex assay (single chip with OVA as reference and six toxin conjugates). 


\section{Materials and methods}

\section{Chemicals, biosensor chips and barley samples}

Flat gold chips (modified with carboxymethylated dextran hydrogel, CMD) were purchased from Xantec (Düsseldorf, Germany). The nanostructured gold chips for iSPR were produced using colloidal lithography and plasma-enhanced chemical vapor deposition (PE-CVD). ${ }^{34}$ In short: poly(methyl methacrylate) (PMMA) was deposited on the glass substrate by PE-CVD using methyl methacrylate as liquid precursor. Spin coating was then used to cover the film with polystyrene (PS) beads (500 $\mathrm{nm}$ with a nominal size dispersion of $10 \%$ ). Next, the sample was exposed to oxygen plasma etching to remove the PMMA from the areas not covered by the beads and to reduce the size of the PS beads to provide the required periodicity. A gold layer of 100-200 nm was then deposited on the surface by physical vapor deposition. Finally, the polystyrene beads were removed resulting in a surface with a gold film perforated by PMMA (Figure $1 \mathrm{C}$ ). The combined control of deposition and etching parameters allowed fine tuning of film thickness and well diameters. The PMMA regions, with diameters of 200-250 nm and periodicity (distance between the centers of the two PMMA wells) of 500-600 nm, make up around $20 \%$ of the total surface area. Detailed characterization of the nanostructured iSPR chips can be found in an earlier publication. ${ }^{35}$

16-Mercaptohexadecanoic acid (MHDA), pentafluorophenol (PFP), N, N'dicyclohexylcarbodiimide (DCC), poly(ethylene glycol) 2-aminoethyl ether acetic acid (average $\mathrm{MW}$ 3500) ( $\left.\mathrm{NH}_{2}-\mathrm{PEG}-\mathrm{COOH}\right)$, ovalbumin (OVA), N-hydroxysuccinimide (NHS), ethanolamine hydrochloride, 1-ethyl-3-(3-dimethylaminopropyl) carbodiimide hydrochloride (EDC), sodium dodecyl sulfate (SDS), Tween20, anhydrous sodium acetate, glacial acetic acid and absolute ethanol were purchased from Sigma Aldrich (Zwijndrecht, The Netherlands). All chemicals were used as obtained, except for dichloromethane (Sigma-Aldrich, $>99.8 \%$ ), which was further purified using a Pure Solv 400 solvent purification system (Innovative Technology, Amesbury, USA). HBS-EP buffer (0.01 M HEPES pH 7.4, $0.15 \mathrm{M} \mathrm{NaCl}, 3 \mathrm{mM}$ EDTA, 0.005\% v/v Surfactant P20) was purchased from GE Healthcare (Uppsala, Sweden). MilliQ water (18.3 M $\Omega \cdot \mathrm{cm}$ resistivity) was obtained using a Merck Millipore (Billerica, USA) water purification system.

The OVA conjugates of mycotoxins (DON-OVA, ZEA-OVA, T-2-OVA, OTA-OVA, FB ${ }_{1}-O V A$ and $\mathrm{AFB}_{1}$-OVA) and monoclonal antibodies (mAbs) against DON (AB-02-22), ZEA (AB-0101 ) and $T-2$ (AB-07-02) were bought from Aokin AG (Berlin, Germany). The mAbs against OTA (SFH-OTA-005) were from Soft Flow Biotechnology (Pecs, Hungary), while the mAbs against $\mathrm{FB}_{1}$ were produced and kindly provided by RIKILT (Wageningen, the Netherlands). The mAbs against AFB1 (12E6) were bought from Europroxima (Arnhem, the Netherlands). Mycotoxin reference solutions of DON, ZEA, T-2, OTA, FB $1, \mathrm{AFB}_{1}$ and 
nivalenol (NIV) were purchased from Biopure via RomerLabs (Tulln, Austria). The other mycotoxins deoxynivalenol-3-glucoside (D3G), 3-acetyl-DON (3ADON), a-zearalanol (a$Z E L), H T-2$, fumonisin $B_{2}$ and $B_{3}\left(F B_{2}\right.$ and $\left.F B_{3}\right)$ were kindly provided by RIKILT.

Barley reference material surpassing the maximum level for ZEA (RMM-01-363a, $729 \pm 244 \mu \mathrm{g} / \mathrm{kg}$ ) was purchased from Aokin AG. The contaminated samples for $\mathrm{T}-2 / \mathrm{HT}-$ 2 sample $(60 \mu \mathrm{g} / \mathrm{kg}$ as measured by LC-MS/MS), for DON (289 $\mu \mathrm{g} / \mathrm{kg}$ as measured by ELISA, $<50 \mu \mathrm{g} / \mathrm{kg}$ as measured by LC-MS/MS) and for OTA ( $25 \mu \mathrm{g} / \mathrm{kg}$ as measured by ELISA, $210 \mu \mathrm{g} / \mathrm{kg}$ as measured by LC-MS/MS) were obtained from project partners. Three different blank samples (containing no mycotoxins based on ELISA or LC-MS/MS pre-screening) were also obtained from project partners. An extraction container and a filtering device were obtained from Abraxis (Warmister, USA) as part of a soil extraction kit.

\section{Surface modification of nanostructured biosensor chips}

The nanostructured iSPR chips were modified with PEG3500 as described previously. ${ }^{35}$ In short: the chips were rinsed with ethanol and water followed by drying with nitrogen. They were then immersed in a $1 \mathrm{mM}$ solution of 16-mercaptohexadecanoic acid (MHDA) in ethanol for $24 \mathrm{~h}$. The MHDA-modified chips were removed, rinsed with ethanol and water, and dried with nitrogen. The acid-terminated chips were activated by immersing overnight in a 1:1 mixture of 0.4 M PFP and 0.4 M DCC in dichloromethane. ${ }^{36}$ The PFPterminated chips were washed with DCM and then immersed in a $1 \mathrm{mg} / \mathrm{mL}$ solution of $\mathrm{NH}_{2}$-PEG-COOH (PEG3500) in DCM for $24 \mathrm{~h}$. The PEG3500-modified chips were removed and rinsed with DCM, sonicated in the same solvent and dried with nitrogen. All the surface modifications were confirmed by comparison with static water contact angle values and XPS results from a previous publication. ${ }^{35}$

\section{Immobilization of mycotoxin-OVA conjugates}

Conventional SPR chips: Ovalbumin (OVA) and mycotoxin-OVA conjugates were immobilized on the carboxymethylated dextran chips using a Biacore 3000 SPR instrument (GE Healthcare, Uppsala, Sweden) as follows: HBS-EP was used as running buffer and the entire immobilization was performed at a constant flow rate of $10 \mu \mathrm{L} / \mathrm{min}$. The microfluidics of the Biacore allows all the channels to be connected in series (Figure 1B) or used separately with their own inlet and outlet. Each channel was individually activated using a 1:1 mixture of $0.4 \mathrm{M}$ EDC and $0.1 \mathrm{M} \mathrm{NHS}$ for $7 \mathrm{~min}$. This was followed by a $7 \mathrm{~min}$ injection of $50 \mu \mathrm{g} / \mathrm{mL}$ of OVA or mycotoxin-OVA conjugate (diluted in $10 \mathrm{mM}$ acetate buffer $\mathrm{pH} 4$ except for ZEA-OVA in $10 \mathrm{mM}$ acetate buffer $\mathrm{pH}$ 3.5) to achieve a response between 3000-5000 (relative to the HBS-EP baseline). For OVA conjugates of DON, T-2 and OTA, a second injection of $50 \mu \mathrm{g} / \mathrm{mL}$ had to be performed to achieve the 
required response. Following the immobilization, the chips were blocked with $1 \mathrm{M}$ ethanolamine, $\mathrm{pH} 8.5$ for $7 \mathrm{~min}$. The chips were then stored at $4-8{ }^{\circ} \mathrm{C}$ until use.

Nanostructured iSPR chips: For the PEG3500-modified nanostructured iSPR chips the immobilization was performed manually on the bench: the entire chip was activated using a 1:1 mixture of 0.1 M NHS and 0.4 M EDC for 20 min followed by washing with water and drying with nitrogen. Two $\mu \mathrm{L}$ of OVA $(1 \mathrm{mg} / \mathrm{mL}$ in $10 \mathrm{mM}$ acetate buffer $\mathrm{pH}$ 3.5) and $2 \mu \mathrm{L}$ of each of the six mycotoxin-OVA conjugates ( $1 \mathrm{mg} / \mathrm{mL}$ of ZEA-OVA, 1 $\mathrm{mg} / \mathrm{mL}$ of $\mathrm{AFB}_{1}$-OVA, $2.8 \mathrm{mg} / \mathrm{mL}$ of T-2-OVA, $2.8 \mathrm{mg} / \mathrm{mL}$ of $\mathrm{FB}_{1}$-OVA, $5 \mathrm{mg} / \mathrm{mL}$ of DONOVA and $3 \mathrm{mg} / \mathrm{mL}$ of OTA-OVA, all in $10 \mathrm{mM}$ acetate buffer $\mathrm{pH} 3.5$ ) were each spotted (manually using a micropipette) on different areas (regions of interest, ROI) of the activated chip. The chip was kept in an air-tight container for $2 \mathrm{~h}$ to avoid evaporation and drying out of the spots. Following the spotting, the chips were washed with water and dried with nitrogen. They were then blocked with ethanolamine for $20 \mathrm{~min}$, rinsed with MilliQ water and dried with nitrogen. The spotted chips were stored at $4-8{ }^{\circ} \mathrm{C}$.

\section{Measurement protocols for double 3-plex assay (SPR) and 6-plex assay (iSPR)}

SPR measurements on carboxymethylated gold chips were performed using a Biacore 3000 and iSPR measurements on PEG3500-modified nanostructured gold chips using a prototype portable imaging nanoplasmonics instrument (Plasmore Srl., Italy). ${ }^{27}$ The channels in the Biacore were connected in series for all measurements (Figure 1B). Typical sensorgrams generated by both instruments can be found in the supporting information (Figure S1). In both formats (double 3-plex with SPR and 6-plex with iSPR), chips were flushed for $1 \mathrm{~min}$ ( $t=0-60 \mathrm{~s}$ ) with running buffer (HBS-EP). The samples were mixed with the antibodies and were injected at a constant flow rate of $20 \mu \mathrm{L} / \mathrm{min}$ for $2 \min (t=60-180 \mathrm{~s}$ ). The chip was then flushed with running buffer (HBS-EP) for 2 $\min (t=180-240 \mathrm{~s})$. The response obtained after 2 min of buffer flow, relative to the starting baseline, was used in calculations. The chip was regenerated by injecting $10 \mathrm{mM}$ $\mathrm{HCl}(100 \mu \mathrm{L} / \mathrm{min})$ for $30 \mathrm{~s}(\mathrm{t}=250-280 \mathrm{~s})$ followed by $20 \mathrm{mM} \mathrm{NaOH}(100 \mu \mathrm{L} / \mathrm{min})$ for 30 $\mathrm{s}(\mathrm{t}=350-380 \mathrm{~s})$. The regeneration was followed by washing with HBS-EP buffer ( 2 min, $t=400-460 \mathrm{~s})$ to remove any remaining regeneration solution. The entire cycle took approximately $15 \mathrm{~min}$. In both SPR and iSPR, OVA was used as a reference, i.e., the response of the OVA channel was subtracted from all the other responses, to correct for any non-specific interactions. The sensorgrams were plotted using Origin 8.5 and Excel 2010. Sensorgrams (for iSPR) were smoothened using the Savitzky-Golay function in Origin 8.5 (10 points of window, polynomial order 2 ). 


\section{Calibration curves, limit of detection and cross-reactivity}

For the calibration curves, a fixed concentration of single or multiple antibodies in HBSEP (without methanol) was mixed with an equal volume of the standard solution containing single or multiple toxins. The toxin standards were diluted in HBS-EP containing $20 \%$ methanol (for singleplex and multiplex assays in buffer) or in barley extract (see below about extraction of barley) in HBS-EP containing $20 \%$ methanol (for multiplex assays in barley). The (i)SPR response obtained for the solution containing both toxins and antibodies $(B)$ relative to the maximum response obtained for the solution containing antibodies only $\left(B_{0}\right)$ was used to calculate the relative response as $\left(B / B_{0}\right) \times 100 \%$. The relative binding was plotted against the concentration of the toxin to obtain the calibration curves. The calibration curves were fitted in GraphPad Prism (Version 6.0, GraphPad Software Inc., USA) using a non-linear four-parameter model to obtain the IC50 values (concentration at which $50 \%$ inhibition of binding occurs). The IC10 values were used as a measure of the limit of detection (LOD) as explained in the results section. Additionally, the IC80 and IC20 values (concentrations at which $80 \%$ and $20 \%$ inhibition of binding occurs, respectively) allowed determination of the working range of the assay. The specificity of the antibodies was tested by determining the crossreactivity of the following modified mycotoxins (masked myctoxins and metabolites) using the calibration curves for the target mycotoxin and modified mycotoxin in buffer as ( IC $_{50}$ target/IC 50 modified mycotoxin) $\times 100 \%$ : D3G, 3ADON, NIV, a-ZEL, FB $2, \mathrm{FB}_{3}, \mathrm{HT}-2$, $\mathrm{AFB}_{2}, \mathrm{AFG}_{1}, \mathrm{AFG}_{2}, \mathrm{AFM}_{1}$.

\section{Extraction of barley samples}

The extraction protocol for blank and naturally contaminated barley samples was based on a previous study. ${ }^{17}$ In short: $2.5 \mathrm{~g}$ of ground barley was weighed in a $50 \mathrm{~mL}$ centrifuge tube. After adding $10 \mathrm{~mL}$ of $80 \%$ methanol, the sample was vortexed vigorously in a Lab dancer (IKA, Staufen, Germany), and shaken for 30 min using an Labquake (Fischer Scientific, Landsmeer, the Netherlands) tube shaker/rotator. Following the extraction, the sample tubes were kept in the fridge for $30 \mathrm{~min}$. Next, the tubes were centrifuged for $10 \mathrm{~min}$ at $4000 \mathrm{~g}$. The supernatant was then collected and diluted four times using HBS-EP buffer solution to obtain an extract in HBS-EP containing $20 \%$ methanol. Finally, the solution was centrifuged for $10 \mathrm{~min}$ at $12000 \mathrm{~g}$ to remove any insoluble components formed as a result of the decrease in organic solvent content. This extract in HBS-EP containing 20\% methanol is referred to as "barley extract" throughout the manuscript and was used for matrix matched calibration curves as well as blank barley for validation experiments. 


\section{Preliminary in-house validation}

In-house validation was performed using 21 blank and 21 spiked barley samples. The 42 samples (blank and spiked) were composed of three different barley batches, one containing $0 \mu \mathrm{g} / \mathrm{kg}$ DON according to ELISA (B1), one with $0 \mu \mathrm{g} / \mathrm{kg}$ OTA according to ELISA (B2) and one mixed barley (B3) selected as blank for all six toxins after LC-MS/MS analysis. For fortification of the samples, the following levels of toxins were used: DON $(1 / 2 \times M L, 625 \mu \mathrm{g} / \mathrm{kg})$, ZEA $(1 / 2 \times M L, 50 \mu \mathrm{g} / \mathrm{kg}), \mathrm{T}-2(1 / 8 \times M L, 25 \mu \mathrm{g} / \mathrm{kg})$, OTA $(2 \times M L, 10$ $\mu \mathrm{g} / \mathrm{kg}), \mathrm{FB}_{1}(1 / 5 \times \mathrm{ML}, 400 \mu \mathrm{g} / \mathrm{kg})$ and $\mathrm{AFB}_{1}(4 \times \mathrm{ML}, 8 \mu \mathrm{g} / \mathrm{kg}) . \mathrm{B} 1, \mathrm{~B} 2$ and B3 (blank and spiked) were each extracted in triplicate and measured on the same day to obtain the intra-day variation. The same experiment was then repeated on two additional days to obtain the inter-day variation. Due to the limited quantity, sample B3 could only be analyzed in triplicate on day 1 and could not be used for inter-day variation (see Scheme $\mathrm{S} 1)$. All the responses shown are relative to the response of a mixed barley (MB) analyzed in triplicate at the beginning of each day. The mixed barley was composed of equal volumes of all the 21 blank barley extracts. For spiked samples, the required volumes of each toxin standard for $2.5 \mathrm{~g}$ of barley were mixed and the toxin mixture (volume not exceeding a total of $50 \mu \mathrm{L}$ ) was spiked on the barley by gently pipetting the mixed solution on the inner side of a $50 \mathrm{~mL}$ centrifuge tube. The solution was mixed with the barley by vigorous vortexing followed by air-drying (left with cap open in the fume hood) for $30 \mathrm{~min}$. The barley samples were then extracted as explained in the extraction section. The obtained barley extracts were mixed with the same volume of an antibody solution. Assuming a $100 \%$ recovery, the mycotoxin concentration in the final solution (in $\mathrm{ng} / \mathrm{mL}$ ) is $1 / 32$ of the original concentration in the barley (in $\mu \mathrm{g} / \mathrm{kg}$ ). Data evaluation was done based on regulations for validation of screening methods. ${ }^{37}$ Validation of a semiquantitative screening method requires identification of a cut-off level (Fm) and threshold value $(T)$. When the SPR response is at or below the cut-off level, the sample is categorized as 'screen positive' and liable to confirmatory analysis. As described in Annex II of the guidelines, ${ }^{37}$ the threshold value $(T)$ was defined as $T=B-1.725 \times \mathrm{SDb}$, where $B$ is the average response of the blank samples, and SDb is the standard deviation of these responses. The cut-off factor $(\mathrm{Fm})$ was defined as $\mathrm{Fm}=\mathrm{M}+1.725 \times \mathrm{SD}$, where $\mathrm{M}$ is the average responses of spiked samples and SD is the standard deviation of these responses. For a successful validation, the Fm value has to be lower than the $\mathrm{T}$ value and only $5 \%$ samples (one of 21 ) are allowed to be false negative.

\section{Naturally contaminated samples}

Naturally contaminated barley samples containing DON $(289 \mu \mathrm{g} / \mathrm{kg}$ as measured by ELISA, $<50 \mu \mathrm{g} / \mathrm{kg}$ as measured by LC-MS/MS), ZEA (CS2, $729 \pm 244 \mu \mathrm{g} / \mathrm{kg}), \mathrm{T}-2 / \mathrm{HT}-2$ (CS3, $60 \mu \mathrm{g} / \mathrm{kg}$ ), and OTA ( $25 \mu \mathrm{g} / \mathrm{kg}$ as measured by ELISA, $210 \mu \mathrm{g} / \mathrm{kg}$ as measured by 
LC-MS/MS) were tested. Three samples were prepared for each contaminated sample measurement: blank barley $(B-)$, spiked barley $(B+)$ and contaminated sample (CS). Each sample $(B-, B+$ and $C S)$ was extracted in triplicate and each extract was measured in triplicate. The following levels of toxins were present in the spiked barley: DON $(1 / 2 \times M L, 625 \mu \mathrm{g} / \mathrm{kg})$, ZEA $(1 / 2 \times M L, 50 \mu \mathrm{g} / \mathrm{kg}), \mathrm{T}-2(1 / 8 \times \mathrm{ML}, 25 \mu \mathrm{g} / \mathrm{kg})$, OTA $(2 \times M L, 10$ $\mu \mathrm{g} / \mathrm{kg}), \mathrm{FB}_{1}(1 / 5 \times \mathrm{ML}, 400 \mu \mathrm{g} / \mathrm{kg})$ and $\mathrm{AFB}_{1}(4 \times \mathrm{ML}, 8 \mu \mathrm{g} / \mathrm{kg})$. All the barley extracts were prepared as described in the experimental section except for ZEA where the contaminated barley extract had to be diluted an additional ten times in $20 \%$ methanol (final concentration of $2.2 \mathrm{ng} / \mathrm{mL}$ assuming $100 \%$ recovery) before $1: 1$ dilution with antibody mixture, to be able to measure within the detection range of the double 3-plex assay. The responses were relative to the response of a mixed blank barley (MB, see section on validation above) analyzed in triplicate prior to each contaminated sample.

\section{Simplified sample preparation for field application}

For possible in-field applications, a simpler extraction protocol was tested with one of the contaminated sample (CS2). A kit (see Figure S2) consisting of a plastic container and a stainless steel ball $(\mathrm{d}=1.5 \mathrm{~cm}$ ) for extraction and a filter device was used. All components of the kit were rinsed several times with $80 \%$ methanol before use. In short: $1.25 \mathrm{~g}$ of ground barley was weighed in the plastic container. After adding $5 \mathrm{~mL}$ of $80 \%$ methanol, the ball was added and the sample was vigorously shaken manually for $1 \mathrm{~min}$. The extract was allowed to stand on the bench for $5 \mathrm{~min}$ and then transferred to the bottom container of the filtration device. A plunger containing the actual filter was attached to the bottom container of the device. Upon applying gentle pressure to the plunger, the extract is filtered into the plunger. The filtrate was collected and diluted four times using HBS-EP buffer solution to obtain an extract in HBS-EP containing $20 \%$ methanol.

\section{Results and discussion}

\section{Double 3-plex benchtop SPR immunoassay development}

Carboxymethylated dextran (CMD) $)^{38,39}$ is the most commonly used surface in SPR and was used in all the Biacore experiments. OVA and OVA conjugates of mycotoxins were immobilized on the CMD surface via amine coupling after one step NHS/EDC activation of the carboxylic acid groups. The covalent attachment of the conjugates is important for re-use and stability of the chips. The six mycotoxins had to be divided over two chips as the Biacore 3000 has four channels, thus allowing the detection of a maximum of three toxins per biosensor chip when using a reference channel. OVA conjugates of DON, ZEA and T-2 were immobilized on one chip as the simultaneous detection of these toxins is 
relevant from an application point of view. On the other chip, $\mathrm{AFB}_{1}$ and OTA were paired together as they both have very low legal limits and might require additional sample treatment. $\mathrm{FB}_{1}$ was the only one remaining and was thus included on the second chip. Once the chips were stable (no significant loss of SPR signal from conjugates after multiple injections of $20 \mathrm{mM} \mathrm{NaOH}$ ), the concentration and buffer composition were optimized for the individual antibodies. HBS-EP containing $20 \%$ methanol was chosen as the dilution buffer for the antibodies during assay development because for most of the antibodies at a fixed concentration, the binding with the immobilized mycotoxin-OVA conjugates was similar (anti-T-2, anti-ZEA, anti-FB ${ }_{1}$, anti-OTA) or higher (for anti-DON and anti-AFB ${ }_{1}$ ) in the methanolic buffer than in pure HBS-EP (Figure S3). Such higher affinity of antibodies in buffer containing methanol has been reported previously in the literature and is not uncommon. ${ }^{32,40}$ Furthermore, for matrix-matched calibration curves in barley, $10 \%$ methanol is present after the extraction and 1:1 mixing with antibodies. Thus, measuring the antibody binding and constructing the calibration curve in HBS-EP containing $10 \%$ methanol is the most appropriate approach. The optimal concentration of the antibodies (corresponding to an SPR response of $100-200$ units) was $0.1 \mu \mathrm{g} / \mathrm{mL}$ for anti-FB $1,1 \mu \mathrm{g} / \mathrm{mL}$ for anti-OTA and anti-ZEA, and $2 \mu \mathrm{g} / \mathrm{mL}$ for anti-T-2, anti-DON and anti-AFB ${ }_{1}$. After optimization of the binding conditions, the regeneration step was optimized. Regeneration is one of the advantages of SPR as it allows reuse of the biosensor chips, unlike most other immunoassay formats. However, for multiplexing applications this is quite challenging since both the binding and regeneration steps have to be optimized for all the different antibodies and the toxins involved. In this study, the most critical antibody-conjugate pairs with respect to regeneration were those for DON, $\mathrm{T}-2$ and $\mathrm{AFB}_{1}$. OVA conjugates of DON and T-2 were sensitive to regeneration and the conjugates on the surface were partly lost according to the SPR response. On the other hand, the antibodies against $A F B_{1}$ were very strongly bound to the chip surface and could not be removed completely upon regeneration. However, after testing a large range of regeneration conditions as recently suggested in literature, ${ }^{41}$ we found that the combination of $10 \mathrm{mM} \mathrm{HCl}$ ( $30 \mathrm{~s}$ at $100 \mu \mathrm{L} / \mathrm{min}$ ) followed by $20 \mathrm{mM} \mathrm{NaOH}$ (30 s at 100 $\mu \mathrm{L} / \mathrm{min}$ ) provided the best compromise for the critical toxins (Figure S4) and the other three toxins (data not shown). Cross-talk of these antibodies with the untargeted mycotoxin conjugates was tested by injecting single antibodies over all the biosensor channels. Some non-specific interaction of anti-T-2 was seen with DON-OVA and ZEAOVA but turned out to be mainly caused by OVA. This could thus be corrected by using the OVA reference channel (see experimental section about measurement protocols), underlining the importance of a suitable reference channel in SPR biosensor immunoassays to avoid misinterpretation of results. 
After optimization of the binding and regeneration conditions, singleplex calibration curves were prepared for all the individual toxins to determine the detection range. These calibration curves (Figure S5) were then compared to the multiplex calibration curves in buffer (Figure $\mathrm{S} 5$ and $2 \mathrm{~A}$ ). The minor differences between the singleplex and multiplex formats show that interference upon mixing different antibodies and toxins was insignificant. Finally, multiplex calibration curves were prepared in barley extract (Figure S5 and $2 \mathrm{~B}$ ) and compared to the multiplex calibration curves in buffer to test for a possible matrix effect. The IC80 values (Table S1) of all toxins (except $\mathrm{AFB}_{1}$ ) in barley extract were only slightly (about two times) higher than in buffer; the most significant difference was seen for DON where the value in barley $(100 \mathrm{ng} / \mathrm{mL})$ was four times higher than the value in buffer $(25 \mathrm{ng} / \mathrm{mL})$. This is expected for calibration curves in sample matrices where several other components contribute to a higher signal than in buffer. In the case of ZEA and OTA, the IC20 and IC50 were not or hardly affected by the sample matrix (barley). For T-2 and $\mathrm{AFB}_{1}$, both the IC20 and IC50 values were affected by the sample matrix. For T-2, the IC20 value for multiplex in barley $(0.02 \mathrm{ng} / \mathrm{mL})$ was five times lower than for multiplex in buffer $(0.1 \mathrm{ng} / \mathrm{mL})$ whereas the IC50 for multiplex in barley $(0.5 \mathrm{ng} / \mathrm{mL})$ was more than two times lower than for multiplex in buffer (1.2 $\mathrm{ng} / \mathrm{mL})$. Similarly, for $\mathrm{AFB}_{1}$, the IC20 value for multiplex in barley $(0.02 \mathrm{ng} / \mathrm{mL})$ was ten times lower than for multiplex in buffer $(0.2 \mathrm{ng} / \mathrm{mL})$ and the IC50 for multiplex in barley $(0.8 \mathrm{ng} / \mathrm{mL})$ was two times lower than for multiplex in buffer $(1.7 \mathrm{ng} / \mathrm{mL})$. Such shifts of the calibration curve in barley towards higher sensitivity, as seen here for T-2 and AFB have also been observed in a multiplex microsphere immunoassay format. ${ }^{17} \mathrm{~A}$ significant difference in the IC50 value was observed for DON where the value in barley $(15 \mathrm{ng} / \mathrm{mL})$ was almost four times higher than the value in buffer $(3.9 \mathrm{ng} / \mathrm{mL})$. For $\mathrm{FB}_{1}$, the only significant difference was in the IC10 values that was almost three times higher for multiplex in buffer $(0.2 \mathrm{ng} / \mathrm{ml})$ compared to multiplex in barley $(0.07 \mathrm{ng} / \mathrm{ml})$. Since all the assays were influenced by the sample matrix (barley) and the effect is not the same for all toxins, matrix-matched calibration curves are recommended prior to measurement of contaminated samples. As seen from the LODs (Table 1), the double 3-plex assay allows detection of all six toxins within the legal requirements of the EU. However, in case of OTA and $\mathrm{AFB}_{1}$, the working range does not allow analysis at ML levels. The performance of the assay, especially for $\mathrm{AFB}_{1}$ and OTA, may be improved by the production of more sensitive antibodies. Alternatively, less dilution of the sample may be considered but at the risk of increased matrix effects and higher methanol concentrations. 
A

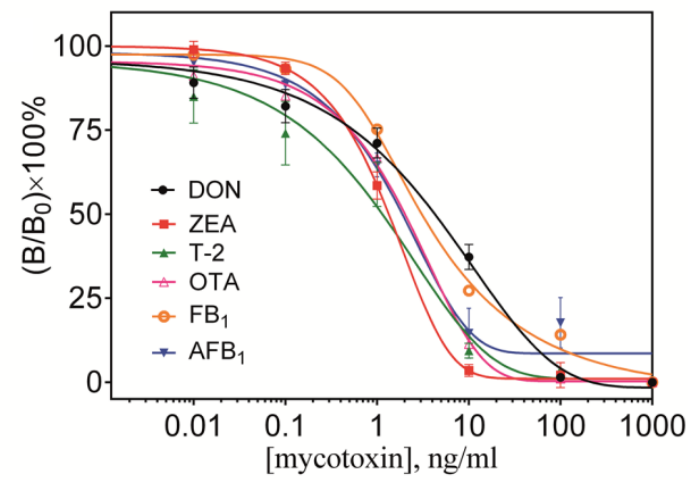

B

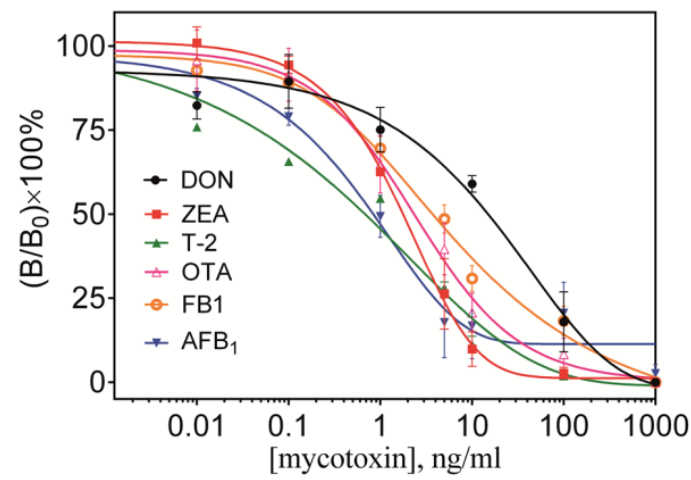

Figure 2. Calibration curves in A) buffer and B) barley extract for the six mycotoxins measured in a double 3-plex format using SPR biosensing (Biacore) $(n=3)$. Two carboxymethylated dextran modified flat gold chips were used: one for DON, T-2 and ZEA and another one for AFB 1 , OTA and $\mathrm{FB}_{1}$.

Table 1. The maximum levels (MLs in $\mu \mathrm{g} / \mathrm{kg}$ ) for the six toxins as specified by the EU, ${ }^{3,4}$ limit of detection (LOD), IC50 and the working range obtained from calibration curves in barley extract measured using the double 3-plex SPR assay and 6-plex nanostructured iSPR assay $(n=3)$. All values are expressed in $\mu \mathrm{g}$ of toxin per $\mathrm{kg}$ of barley.

\begin{tabular}{llllllll}
\hline \multirow{2}{*}{ Toxins } & \multirow{2}{*}{ MLS } & \multicolumn{3}{c}{ Double 3-plex SPR } & \multicolumn{3}{l}{ 6-plex nanostructured iSPR } \\
\cline { 3 - 8 } & & LOD $^{\mathrm{a}}$ & IC50 & Working range & LOD $^{\mathrm{a}}$ & IC50 & Working range $^{\mathrm{b}}$ \\
\hline DON & 1250 & $26^{*}$ & 480 & $26-3200$ & 64 & 1220 & $192-4800$ \\
ZEA & 100 & 6 & 51 & $16-160$ & 96 & 960 & $224-8000$ \\
T-2 & $200^{\mathrm{c}}$ & $0.6^{*}$ & 16 & $0.6-290$ & 26 & 580 & $80-3800$ \\
OTA & 5 & 3 & 67 & $13-320$ & 160 & 1280 & $320-5700$ \\
FB $_{1}$ & $2000^{\mathrm{d}}$ & 2 & 112 & $10-1200$ & 13 & 480 & $48-3800$ \\
AFB $_{1}$ & 2 & 0.6 & 26 & $3-260$ & 10 & 640 & $48-8000$
\end{tabular}

a LOD is defined as IC10 (concentration at which $10 \%$ inhibition of binding occurs) unless marked with an asterisk

${ }^{\text {b}}$ Working range is defined as IC20-IC80 (concentration at which $20 \%-80 \%$ inhibition of binding occurs)

${ }^{\mathrm{C}}$ Recommendation for sum of T-2 and HT-2

dSum of FB1 and FB2 in unprocessed maize

*IC20 is used as LOD, as the fitting does not allow determination of IC10 for this toxin

Direct comparison of the sensitivity of the double 3-plex SPR assay with literature values is complicated due to differences in assay types, antibodies, sample matrices, sample preparation as well as the calculation method for determination of the limit of detection (LOD). Nevertheless, the most relevant literature values reported for different mycotoxins have been compiled in Table 2. The reported LODs are often calculated by 
subtracting three times the standard deviation from the average blank response. ${ }^{30,31}$ Due to the very low noise of the Biacore instrument, the signal corresponding to such an LOD is at $1-5 \%$ relative inhibition. The standard deviations of the blank sample of these reported SPR assays are already higher than the noise of the Biacore, making such LOD calculations less preferable. ${ }^{30,31}$ Therefore, IC10 values (10\% inhibition of binding) were used in the present work as a reasonable estimate of sensitivity (LOD). In case of DON and T-2, IC20 values were used as LOD because the fitting of the calibration curve did not allow determination of IC10 values. Compared to another SPR study on detection of DON and T-2/HT-2, our assay is comparable for DON while being more sensitive for T2/HT-2. ${ }^{30,31}$ Compared to the reported values of a previous multiplex SPR study, our assay may be judged less sensitive for all toxins except $\mathrm{FB}_{1}$ and $\mathrm{AFB}_{1} .{ }^{33}$ However, such a comparison is unjustified as the method for calculating the LOD is not described in ref. 33 and the calibration curves are not shown in matrix (nevertheless the LODs are reported in $\mathrm{ng} / \mathrm{g}$ for "sample" without specification of the sample ${ }^{33}$ ). In case of $\mathrm{AFB}_{1}$, the reported sensitivity ${ }^{33}$ is comparable to our assay. The most significant difference can be seen in our $\mathrm{FB}_{1}$ assay, which is 25 times more sensitive than the previous SPR assay. ${ }^{33}$ For $\mathrm{AFB}_{1}$, our assay in buffer is almost ten times more sensitive than another SPR assay reported in literature. ${ }^{29}$ For OTA in cereals, our assay is ten times less sensitive than the assay in a previous report. ${ }^{32}$ This can be easily explained by the gold nanoparticles used in ref. 32 for SPR signal enhancement as the authors claim a 17-fold increase in sensitivity due to the nanoparticles. Compared to benchtop iSPR, ${ }^{26}$ our benchtop SPR assay is about 2-3 times more sensitive for DON and 6-10 times more sensitive for ZEA.

Table 2. SPR detection limits of mycotoxins in buffer (in $\mathrm{ng} / \mathrm{mL}$ ) and in different matrices (in $\mu \mathrm{g} / \mathrm{kg}$ ) reported in the literature. Sensitivity of the present work can be found in Table 1 and Table S1.

\begin{tabular}{lllll}
\hline \multirow{2}{*}{ Toxins } & \multicolumn{2}{l}{ Buffer $(\mathrm{ng} / \mathrm{mL})$} & \multicolumn{2}{l}{ Matrix $(\mu \mathrm{g} / \mathrm{kg})$} \\
\cline { 2 - 5 } & Singleplex & Multiplex & Singleplex & Multiplex \\
\hline DON & - & - & - & $0.5,1-29,68-84^{26,31,33}$ \\
ZEA & - & - & - & $0.01,40-64^{26,33}$ \\
T-2 & - & - & $26^{30}$ & $31-47^{31}$ \\
OTA & - & - & $0.06-0.5^{32}$ & $0.1^{33}$ \\
FB $_{1}$ & - & - & - & $50^{33}$ \\
AFB $_{1}$ & $3.0^{29}$ & - & - & $0.2^{33}$ \\
\hline
\end{tabular}




\section{Cross-reactivity with modified mycotoxins}

The double 3-plex immunoassay was tested with modified mycotoxins that commonly occur in food and feed (Table 3). The antibodies against DON showed significant crossreactivity towards D3G and 3ADON as seen in previous reports. ${ }^{31}$ However, in contrast with literature, no cross-reactivity was seen with NIV in our case. This could be due to the use of polyclonal antibodies in the earlier studies compared to monoclonal antibodies used here. Such lack of cross-reactivity with NIV has been seen in other reports where antibodies similar to ours were used. ${ }^{17,26}$ Antibodies against ZEA (anti-ZEA) were crossreactive towards $a-Z E L$ and the anti-FB $B_{1}$ towards $\mathrm{FB}_{2}$ and $\mathrm{FB}_{3}$. The measured crossreactivity of anti-T-2 towards $\mathrm{HT}-2$ has been reported earlier. ${ }^{30,31}$ According to ELISA studies performed by the supplier and the data provided, antibodies against $\mathrm{AFB}_{1}$ show cross-reactivities towards $A F B_{2}, A F G_{1}, A F G_{2}$ and $A F M_{1}$. Such cross-reactivities from ELISA have also been reported in earlier literature. ${ }^{29}$ The observed cross-reactivity might lead to overestimation of the targeted mycotoxin concentration in real samples. Note that in some cases, such as $\mathrm{T}-2$ and $\mathrm{HT}-2, \mathrm{FB}_{1}$ and $\mathrm{FB}_{2}$, and the aflatoxins (data obtained using ELISA from Europroxima), the cross-reactivity is desirable as the regulations specify the summed toxin concentration. In other cases such as DON, ZEA and $A_{F B}$ where the cross-reactivity leads to overestimation of target mycotoxin, the "suspect" samples can be subjected to further quantitative LC-MS/MS analysis.

Table 3. IC50 values and corresponding cross-reactivities of mycotoxin metabolites measured in singleplex format using SPR (Biacore) $(n=3)$.

\begin{tabular}{llllll}
\hline Metabolite & $\begin{array}{l}\text { IC50 } \\
(\mathrm{ng} / \mathrm{mL})\end{array}$ & $\begin{array}{l}\text { Cross-reactivity } \\
(\%)\end{array}$ & Metabolite & $\begin{array}{l}\text { IC50 } \\
(\mathrm{ng} / \mathrm{mL})\end{array}$ & $\begin{array}{l}\text { Cross-reactivity } \\
(\%)\end{array}$ \\
\hline DON & 5.6 & $100 \pm 7$ & $\mathrm{FB}_{1}$ & 2.7 & $100 \pm 9$ \\
D3G & 10 & $56 \pm 5$ & $\mathrm{FB}_{2}$ & 2.6 & $104 \pm 2$ \\
3ADON & 5.0 & $112 \pm 12$ & $\mathrm{FB}_{3}$ & 2.0 & $135 \pm 4$ \\
NIV & $\mathrm{ND}$ & $<1$ & & & \\
\hline ZEA & 1.5 & $100 \pm 8$ & $\mathrm{~T}-2$ & 1.9 & $100 \pm 8$ \\
a-ZEL & 1.9 & $79 \pm 5$ & $\mathrm{HT}-2$ & 2.5 & $76 \pm 5$ \\
\hline $\mathrm{AFB}_{1} *$ & 0.1 & 100 & & & \\
$\mathrm{AFB}_{2} *$ & 0.6 & 17 & & & \\
$\mathrm{AFG}_{1} *$ & 0.7 & 14 & & & \\
$\mathrm{AFG}_{2} *$ & 1.1 & 9 & & & \\
$\mathrm{AFM}_{1} *$ & 1.1 & 9 & & & \\
\hline $\mathrm{ND}^{*}$ not detectable & & & & \\
$*$ Determined by ELISA & & & &
\end{tabular}




\section{Durability of SPR chips following multiple analyses and regenerations}

All three calibration curves for the double 3-plex SPR assay (singleplex in buffer, multiplex in buffer, multiplex in barley extract) were measured in triplicate using the same concentration of antibodies on the same chip. The minor differences between the first (singleplex in buffer) and last calibration curve (multiplex in barley extract) showed that the chips can be re-used at least 60 times.

\section{Preliminary in-house validation of the double 3-plex SPR assay}

For validation, 21 blank barley and 21 spiked barley samples were measured using the double 3-plex SPR assay. The following levels of toxins were used for spiking of barley:

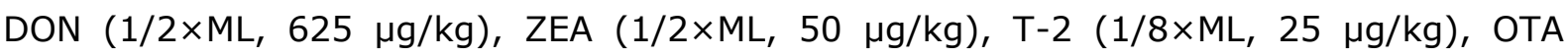
$(2 \times M L, 10 \mu \mathrm{g} / \mathrm{kg}), \mathrm{FB}_{1}(1 / 5 \times M L, 400 \mu \mathrm{g} / \mathrm{kg})$ and $\mathrm{AFB}_{1}(4 \times M \mathrm{~L}, 8 \mu \mathrm{g} / \mathrm{kg})$. For four toxins (DON, ZEA, T-2 and $\mathrm{FB}_{1}$ ), the cut-off factor ( $\mathrm{Fm}$ ) was smaller than the threshold value (T) (Figure S6) as required for a successful validation (see experimental section). ${ }^{37}$ The concentrations corresponding to the average measured response were $22 \%$ lower for DON, $24 \%$ lower for ZEA, $28 \%$ lower for T-2 and $26 \%$ lower for $\mathrm{FB}_{1}$, when compared to the spiked levels probably due to incomplete extraction. Despite this, inhibition was seen for all four toxins in 19 out of 21 samples. These samples would be considered "suspect" in our screening test and would be subjected to further quantitative analysis by LCMS/MS. For OTA and $\mathrm{AFB}_{1}$, based on the calibration curve, validation the relevant MLS is not possible as discussed earlier. Based on the results obtained at $2 \times M L$ for OTA and $4 \times M L$ for $A_{F B}$ (data not shown), the current double 3-plex assay would require levels of approximately $5 \times M L$ for OTA and $20 \times M L$ for $A_{F B}$ for a successful validation $(F m<T)$.

The inter-day and intra-day precision (RSD in \%) of the assay were calculated as the standard deviation of the data discussed above and are depicted in Table 4. Since the 21 samples were divided over three different days, the average values of the different inter- and intra-day RSDs are reported. In all cases, as expected the inter-day RSDs are higher than the intra-day. The RSDs of our assay (comparable to previous SPR assays ${ }^{30,31}$ ) demonstrate that the assay is fit-for-purpose.

Table 4. Inter- and intraday precision of the mycotoxin assay for blank and spiked barley samples measured with double 3-plex SPR assay.

\begin{tabular}{llllllll}
\hline \multirow{2}{*}{ Sample } & Precision & \multicolumn{7}{l}{ RSD for each assay in \% } \\
\cline { 3 - 8 } Blank & Intra-day $(n=21)$ & 5.4 & 3.6 & 3.1 & 1.8 & 4.4 & 1.5 \\
& Inter-day $(n=18)$ & 11.7 & 9.4 & 8.9 & 4.6 & 7.1 & 5.5 \\
\hline \multirow{2}{*}{ Spiked } & Intra-day $(n=21)$ & 0.9 & 4.3 & 1.2 & 3.4 & 4.3 & 5.2 \\
& Inter-day $(n=18)$ & 9.4 & 9.6 & 9.9 & 6.2 & 7.2 & 11.4 \\
\hline
\end{tabular}




\section{Measurement of naturally contaminated barley samples using the double 3-plex SPR assay}

To demonstrate the application of the double 3-plex assay, naturally contaminated barley samples containing DON (CS1, $289 \mu \mathrm{g} / \mathrm{kg}$ as measured by ELISA, $<50 \mu \mathrm{g} / \mathrm{kg}$ as measured by LC-MS/MS), ZEA (CS2, $729 \pm 244 \mu \mathrm{g} / \mathrm{kg}), \mathrm{T}-2 / \mathrm{HT}-2(\mathrm{CS} 3,60 \mu \mathrm{g} / \mathrm{kg})$ and OTA (CS4, $25 \mu \mathrm{g} / \mathrm{kg}$ as measured by ELISA, $210 \mu \mathrm{g} / \mathrm{kg}$ as measured by LC-MS/MS) were tested. Compared to the mixed blank used for normalization and the blank samples samples (B-), all the contaminated samples (CS) showed significant inhibition (Figure 3), thus proving the applicability of the double 3-plex assay for screening of naturally contaminated samples. For naturally contaminated barley samples containing DON, the measured concentration is $27 \%$ lower than the value obtained by ELISA. Although the sample was considered as blank by LC-MS/MS, the higher concentration measured in our assay could be due to the presence of other modified forms of DON $(<100 \mu \mathrm{g} / \mathrm{kg} \mathrm{DON3G}$, $<40 \mu \mathrm{g} / \mathrm{kg} 3$ ADON and $<40 \mu \mathrm{g} / \mathrm{kg}$ 15ADON according to LC-MS/MS analysis) and their cross-reactivity with anti-DON used in the study. The measured concentration for naturally contaminated samples containing OTA is only $36 \%$ lower than values obtained by ELISA. But the LC-MS/MS value for the sample is $800 \%$ higher than measured by ELISA. Although the reason for such a variation is not completely clear, such differences between values measured by ELISA and LC-MS/MS have been reported earlier in the literature ${ }^{42}$ and may be caused by sample inhomogeneity or other discrepancies between the sample matrix and the blank matrix used for calibration. Compared to the known values, the measured concentrations are about $21 \%$ lower for ZEA and $46 \%$ lower for T2. The lower measured concentrations in naturally contaminated samples of ZEA, T-2 and OTA are probably partially due to incomplete extraction recovery (see validation results). In contrast to LC-MS/MS analysis where a stable isotope internal standard can be used, no correction for incomplete recovery is possible in our case. Therefore, a matrix matched calibration curve would be required before analysis of contaminated samples. Naturally contaminated samples for the other two mycotoxins ( $F B_{1}$ and $A F B_{1}$ ) were not available and therefore not included in the present study. 

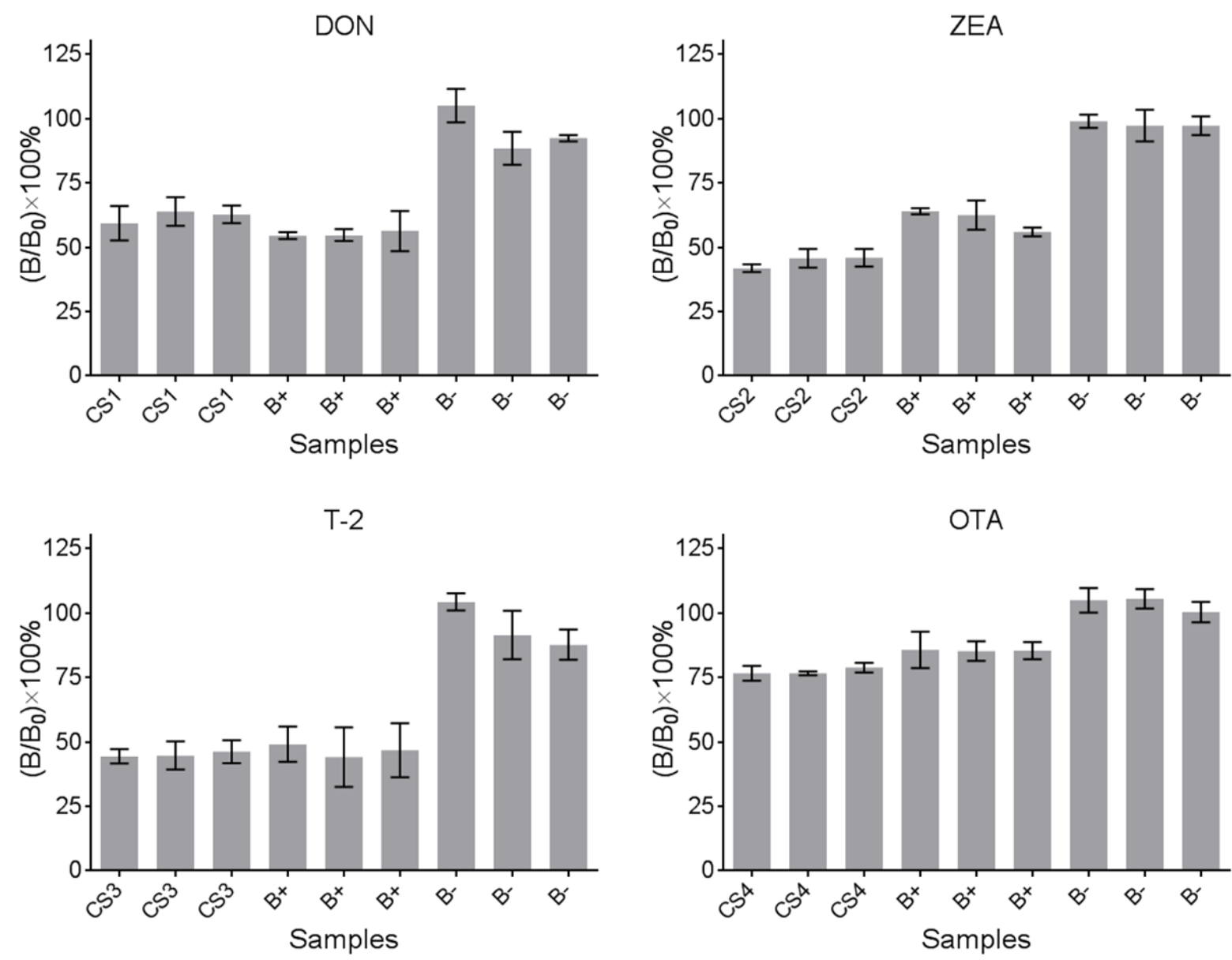

Figure 3. Relative responses measured for naturally contaminated barley samples for presence of DON, ZEA, T-2 and OTA using double 3-plex benchtop SPR (Biacore). Three types of barley extract were used for each contaminated sample measurement: blank barley $(B-)$, spiked barley $(B+)$ and contaminated sample (CS). Each sample (B-, B+ and CS) were extracted in triplicate and each extract was measured in triplicate. The responses are relative to the response of a mixed blank barley. The error bars are the standard deviation of triplicate responses of each extract.

\section{Transferability of the double 3-plex SPR assay to a 6-plex assay on a prototype portable iSPR instrument}

One of the hurdles for SPR to be used in field applications is the size and weight of the instrument. Therefore, a prototype portable iSPR instrument with nanostructured iSPR chips, previously used to study the antifouling properties of biosensor chips ${ }^{35}$ and demonstrated in a medical application ${ }^{27}$, was tested for the transferability of the developed assay. OVA and the same six mycotoxin-OVA conjugates as used in the benchtop SPR assay were covalently immobilized on a PEG3500-modified nanostructured iSPR chip. PEG3500 was chosen because of its well-characterized antifouling properties. ${ }^{35}$ Although higher concentrations (50-200 times) of conjugates were used during the immobilization on the iSPR chips, manual spotting required much lower sample volumes $(2 \mu \mathrm{L})$ compared to the Biacore $(100 \mu \mathrm{L})$. The optimal concentrations of the antibodies 
(corresponding to an SPR response of 0.04-0.05 units) in the 6-plex assay were $5 \mu \mathrm{g} / \mathrm{mL}$ for $\mathrm{FB}_{1}$ and $50 \mathrm{\mu g} / \mathrm{mL}$ for OTA and ZEA, T-2, DON, and $\mathrm{AFB}_{1}$. Note that the nanoplasmonics iSPR assay required higher concentrations of antibodies (50 times for $\mathrm{FB}_{1}$, OTA and ZEA and 25 times for DON, T-2 and AFB ${ }_{1}$ ) relative to the double 3-plex SPR (see above), which is due to two main factors: difference in surface chemistry of the two surfaces and hardware sensitivity. The iSPR chips are coated with a 2D polymer (PEG3500) having fewer binding groups $(\mathrm{COOH})$ compared to the 3D hydrogel (CMD) in the double 3-plex SPR set-up. This limits the amount of mycotoxin conjugates that can be immobilized on the surface, thus requiring higher concentrations of antibodies. Furthermore, as seen in our previous study, the sensitivity of the prototype iSPR instrument is approximately ten times lower than that of the Biacore, ${ }^{35}$ thus more antibodies are required to give a detectable response in the inhibition assay.

For the double 3-plex SPR assay (Biacore), the singleplex calibration curves in buffer were rather similar to the multiplex calibration curves in buffer (Figure S5). Upon measuring $\mathrm{FB}_{1}$ calibration curves using the prototype nanostructured iSPR instrument, the two calibration curves were again similar. Extrapolating on this, for the other five toxins only multiplex calibration curves in buffer and barley extract were measured in the prototype iSPR (Figure S7 and Figure 4). As expected, the calibration curves indicate a decrease in sensitivity compared to the double 3-plex SPR assay. For most toxins, the sensitivity (Table 1 ) is about 3-20 times less than for the Biacore and similar to a benchtop iSPR instrument (for DON and ZEA), ${ }^{26}$ except for OTA and T- 2 where the iSPR assay is almost 40 and 50 times less sensitive than the benchtop SPR assay, respectively. The difference could be due to the amount of OVA conjugates immobilized on the surface. Although the spots can be visualized in the imaging instrument, the actual amount of immobilized conjugates cannot be as easily checked as in the Biacore. Note that even in the benchtop SPR assay (Biacore), a second injection of OTA-OVA and T-2-OVA was required to achieve a good response. For four toxins (DON, ZEA, T-2 and $\mathrm{FB}_{1}$ ), the 6-plex assay is sensitive enough to detect the toxins at MLs as defined by EU legislation. ${ }^{3,4}$ In case of OTA and $\mathrm{AFB}_{1}$, better binding antibodies will be required to obtain the required sensitivity. One of the contaminated samples containing $729 \pm 244$ $\mu \mathrm{g} / \mathrm{kg}$ of ZEA (22.7 $\mathrm{ng} / \mathrm{ml}$ assuming $100 \%$ recovery) was also measured using the prototype nanoplasmonics iSPR instrument. Although, the measured concentration was $31 \%$ lower than stated, inhibition was clearly seen relative to the mixed blank barley sample (Figure S8). To demonstrate the applicability of the assay as a screening method for in-field applications, the same contaminated sample was extracted using a simpler extraction protocol (see experimental section on naturally contaminated samples. Both extraction protocols gave similar results (Figure S8) showing the potential of such an extraction protocol for future field applications. The simplified sample preparation is both 
time and cost effective as it required less equipment and expert personnel. The performance of the portable iSPR assay may be further improved by using a 3D hydrogel chip surface comparable to CMD or by functionalized zwitterionic polymer brushes. Another approach for sensitivity improvement could be based on confinement-induced signal enhancement. ${ }^{43,44}$ In a nanostructured chip, in addition to the bulk SPR due to the propagation of surface plasmons, localized SPR (LSPR) occurs. The LSPR mode has been reported to be eight times more sensitive than the bulk SPR mode. ${ }^{27}$ The maximum of the evanescent wave from LSPR is at the top of the PMMA wells as described in ref. 44. Therefore, controlled and precise filling of these wells in such a way that the OVA-toxin conjugates are exactly near the top of the well after immobilization, may help to boost sensitivity. The performance of the prototype instrument itself can be improved by upgrading the hardware.
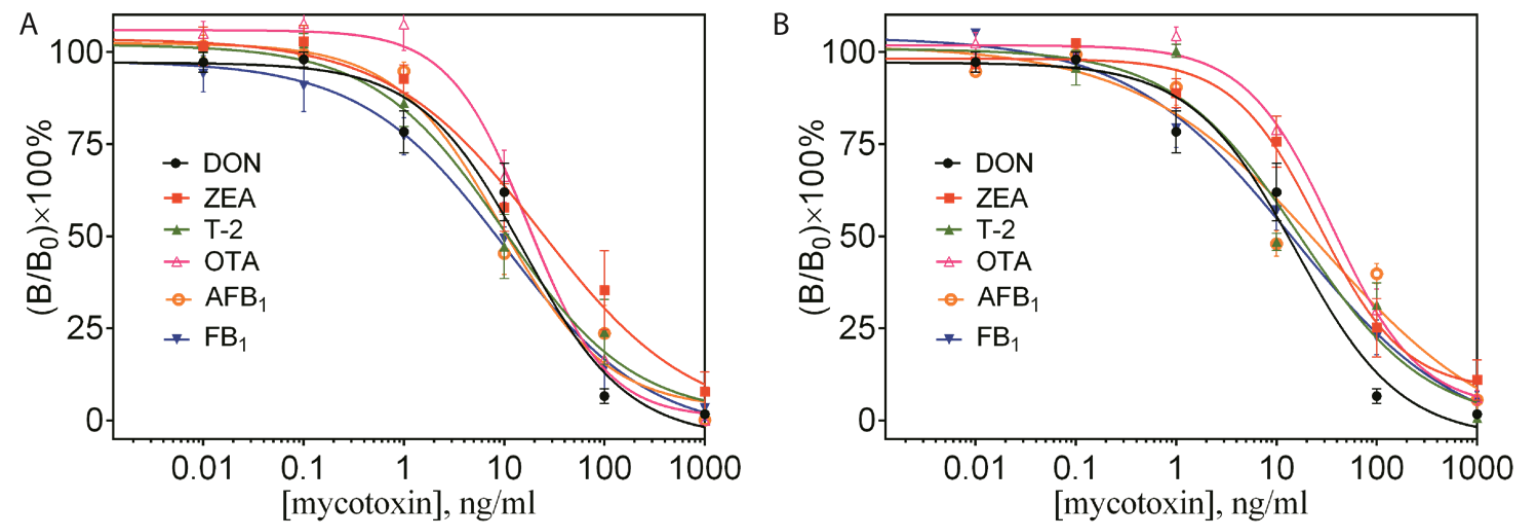

Figure 4. Calibration curves in A) buffer and B) barley extract for the six mycotoxins measured in a 6-plex format using a prototype nanostructured iSPR instrument. A single PEG3500 chip was used for detection of DON, ZEA, T-2, OTA, FB 1 and $\mathrm{AFB}_{1}$.

The benchtop SPR and prototype nanostructured iSPR each have their own advantages and disadvantages. While nanostructured iSPR is in this application less sensitive than benchtop SPR, it allows simultaneous detection of six toxins using one chip. This would be beneficial for rapidly detecting both targeted and other less frequently occurring mycotoxins in real samples. Additionally, if the sensitivity could be improved, nanostructured iSPR would save costs in terms of the chip, reagents as well as assay time. However, it should be kept in mind that optimization of regeneration conditions can be difficult in a one-chip format. In a double 3-plex format with benchtop SPR, regeneration-sensitive conjugates (such as DON-OVA and T-2-OVA) can be separated from the conjugates that are more resistant to regeneration (such as $\mathrm{AFB}_{1}$-OVA and $\mathrm{FB}_{1}$ OVA). The toxins can also be separated based on their required detection levels set by EU. For some toxins, such as DON and $\mathrm{FB}_{1}$, diluted sample extracts would be desirable, whereas for others (OTA and $\mathrm{AFB}_{1}$ ) concentrated extracts would be desirable. 


\section{Conclusions}

Mycotoxin contamination of food and feed is a major concern for producers and quality controllers. Most published mycotoxin detection studies have focused on the detection of one or a few mycotoxins. In addition to multiplex detection, there is also a demand for miniaturized instruments to bring the lab to the sample. However, a benchmark to properly evaluate such prototype miniaturized instruments is missing from the literature. This work provides such a comprehensive study for the multiplex detection of six of the most relevant mycotoxins. The developed benchmark method allows detection of deoxynivalenol (DON), zearalenone (ZEA), T-2 toxin, ochratoxin A (OTA), fumonisin $B_{1}$ $\left(\mathrm{FB}_{1}\right)$ and aflatoxin $\mathrm{B}_{1}\left(\mathrm{AFB}_{1}\right)$ in a double 3-plex format using a benchtop SPR (Biacore) with two separate SPR biosensor chips. The detection range of the assays allows the detection of four mycotoxins ( $D O N$, ZEA, T-2 and $\mathrm{FB}_{1}$ ) in barley at the regulatory limits. For OTA and $\mathrm{AFB}_{1}$, further improvement in sensitivity, either by using better antibodies or using less diluted samples, is still required for screening at the regulatory limits. The sensitivity of our assay is comparable to the reported literature values for singleplex SPR assays, but offers the benefit of multiplexing. In case of $\mathrm{FB}_{1}$, our assay is 25 times more sensitive than earlier SPR assays. The validation and measurement of contaminated samples show that the recovery of the toxins is about 75\%. Thus, the developed double 3-plex SPR assay could serve well as a semi-quantitative screening method, to be followed up by further analysis of any "suspected" samples using LC-MS/MS. Finally, the transfer to a prototype portable iSPR instrument showed the simultaneous detection of all six mycotoxins in a 6-plex format using a single nanostructured iSPR chip. The prototype iSPR sensitivity, although less than that of the benchtop instrument (Biacore), could be improved by different strategies including change of surface chemistry, selection of better antibodies and hardware upgrades. The demonstrated transferability provides a step forward towards use of a miniaturized iSPR for at-line and in-field biosensing screening of mycotoxins in barley.

\section{Acknowledgements}

This research received funding from the Netherlands Organisation for Scientific Research (NWO) in the framework of the Technology Area COAST (SPRing, project nr 053.21.107) with WU, VU Amsterdam, RIKILT, Heineken, Synthon, Technex, EuroProxima, Waterproef as partners and Plasmore and Bionavis as associated partners. We would like to thank these project partners and especially Willem Haasnoot from RIKILT and Ron Verheijen, Nermin Sajic and Piet van Wichen from EuroProxima, for stimulating discussions, supply of sample materials and suggestions for initial experiments. 


\section{References}

1. DeVries, J. W.; Trucksess, M. W.; Jackson, L. S., Mycotoxins and food safety. Springer: New York, 2002.

2. Schatzmayr, G.; Streit, E., Global occurrence of mycotoxins in the food and feed chain: facts and figures. World Mycotoxin J. 2013, 6, 213-222.

3. Commission regulation (EC) No 1881/2006 of 19 December 2006 setting maximum levels for certain contaminants in foodstuffs. Off. J. Eur. Union 2006, L364, 5-24.

4. Marmisollé, W. A.; Capdevila, D. A.; de la Llave, E.; Williams, F. J.; Murgida, D. H., SelfAssembled Monolayers of $\mathrm{NH}_{2}$-Terminated Thiolates: Order, pKa, and Specific Adsorption. Langmuir 2013, 29, 5351-5359.

5. McGrath, T. F.; Elliott, C. T.; Fodey, T. L., Biosensors for the analysis of microbiological and chemical contaminants in food. Anal. Bioanal. Chem. 2012, 403, 75-92.

6. Vidal, J. C.; Bonel, L.; Ezquerra, A.; Hernández, S.; Bertolín, J. R.; Cubel, C.; Castillo, J. R., Electrochemical affinity biosensors for detection of mycotoxins: A review. Biosens. Bioelectron. 2013, 49, 146-158.

7. Berthiller, F.; Burdaspal, P. A.; Crews, C.; Iha, M. H.; Krska, R.; Lattanzio, V. M. T.; MacDonald, S.; Malone, R. J.; Maragos, C.; Solfrizzo, M.; Stroka, J.; Whitaker, T. B., Developments in mycotoxin analysis: An update for 2012-2013. World Mycotoxin J. 2014, 7, 3-33.

8. Berthiller, F.; Brera, C.; Crews, C.; Iha, M. H.; Krsha, R.; Lattanzio, V. M. T.; MacDonald, S.; Malone, R. J.; Maragos, C.; Solfrizzo, M.; Stroka, J.; Whitaker, T. B., Developments in mycotoxin analysis: An update for 2013-2014. World Mycotoxin J. 2015, 8, 5-35.

9. Berthiller, F.; Sulyok, M.; Krska, R.; Schuhmacher, R., Chromatographic methods for the simultaneous determination of mycotoxins and their conjugates in cereals. Int. J. Food Microbiol. 2007, 119, 33-37.

10. Todescato, F.; Antognoli, A.; Meneghello, A.; Cretaio, E.; Signorini, R.; Bozio, R., Sensitive detection of ochratoxin $A$ in food and drinks using metal-enhanced fluorescence. Biosens. Bioelectron. 2014, 57, 125-132.

11. Yang, L.; Zhang, Y.; Li, R.; Lin, C.; Guo, L.; Qiu, B.; Lin, Z.; Chen, G., Electrochemiluminescence biosensor for ultrasensitive determination of ochratoxin $A$ in corn samples based on aptamer and hyperbranched rolling circle amplification. Biosens. Bioelectron. 2015, 70, 268-274.

12. Zangheri, M.; Di Nardo, F.; Anfossi, L.; Giovannoli, C.; Baggiani, C.; Roda, A.; Mirasoli, M., A multiplex chemiluminescent biosensor for type B-fumonisins and aflatoxin B1 quantitative detection in maize flour. Analyst 2015, 140, 358-365.

13. Liu, X.; Xu, Y.; Xiong, Y.-h.; Tu, Z.; Li, Y.-p.; He, Z.-y.; Qiu, Y.-I.; Fu, J.-h.; Gee, S. J.; Hammock, B. D., VHH phage-based competitive real-time immuno-polymerase chain reaction for ultrasensitive detection of ochratoxin A in cereal. Anal. Chem. 2014, 86, 7471-7477.

14. Olcer, Z.; Esen, E.; Muhammad, T.; Ersoy, A.; Budak, S.; Uludag, Y., Fast and sensitive detection of mycotoxins in wheat using microfluidics based real-time electrochemical profiling. Biosens. Bioelectron. 2014, 62, 163-169.

15. Beloglazova, N. V.; Speranskaya, E. S.; Wu, A.; Wang, Z.; Sanders, M.; Goftman, V. V.; Zhang, D.; Goryacheva, I. Y.; De Saeger, S., Novel multiplex fluorescent immunoassays based on quantum dot nanolabels for mycotoxins determination. Biosens. Bioelectron. 2014, 62, 59-65.

16. Peters, J.; Bienenmann-Ploum, M.; de Rijk, T.; Haasnoot, W., Development of a multiplex flow cytometric microsphere immunoassay for mycotoxins and evaluation of its application in feed. Mycotoxin Res. 2011, 27, 63-72.

17. Peters, J.; Cardall, A.; Haasnoot, W.; Nielen, M. W. F., 6-Plex microsphere immunoassay with imaging planar array detection for mycotoxins in barley. Analyst 2014, 139, 3968-3976.

18. Vaclavik, L.; Zachariasova, M.; Hrbek, V.; Hajslova, J., Analysis of multiple mycotoxins in cereals under ambient conditions using direct analysis in real time (DART) ionization coupled to high resolution mass spectrometry. Talanta 2010, 82, 1950-1957.

19. Goryacheva, I. Y.; De Saeger, S., Immunochemical methods for rapid mycotoxin detection in food and feed. In Determining mycotoxins and mycotoxigenic fungi in food and feed, De Saeger, S., Ed. Woodhead Publishing: Cambridge, 2011; pp 135-167.

20. Song, S.; Liu, N.; Zhao, Z.; Njumbe Ediage, E.; Wu, S.; Sun, C.; De Saeger, S.; Wu, A., Multiplex lateral flow immunoassay for mycotoxin determination. Anal. Chem. 2014, 86, 4995-5001.

21. Li, Y.; Liu, X.; Lin, Z., Recent developments and applications of surface plasmon resonance biosensors for the detection of mycotoxins in foodstuffs. Food Chem. 2012, 132, 1549-1554. 
22. Meneely, J. P.; Elliott, C. T., Rapid surface plasmon resonance immunoassays for the determination of mycotoxins in cereals and cereal-based food products. World Mycotoxin J. 2014, 7, 491-505.

23. Homola, J., Surface plasmon resonance sensors for detection of chemical and biological species. Chem. Rev. 2008, 108, 462-493.

24. Yeatman, E.; Ash, E. A., Surface plasmon microscopy. Electron. Lett. 1987, 23, 1091-1092.

25. Scarano, S.; Mascini, M.; Turner, A. P. F.; Minunni, M., Surface plasmon resonance imaging for affinity-based biosensors. Biosens. Bioelectron. 2010, 25, 957-966.

26. Dorokhin, D.; Haasnoot, W.; Franssen, M. C. R.; Zuilhof, H.; Nielen, M. W. F., Imaging surface plasmon resonance for multiplex microassay sensing of mycotoxins. Anal. Bioanal. Chem. 2011, 400, 3005-3011.

27. Bottazzi, B.; Fornasari, L.; Frangolho, A.; Giudicatti, S.; Mantovani, A.; Marabelli, F.; Marchesini, G.; Pellacani, P.; Therisod, R.; Valsesia, A., Multiplexed label-free optical biosensor for medical diagnostics. J. Biomed. Opt. 2014, 19, 017006-017010.

28. Dahlin, A. B., Sensing applications based on plasmonic nanopores: The hole story. Analyst 2015, 140, 4748-4759.

29. Daly, S. J.; Keating, G. J.; Dillon, P. P.; Manning, B. M.; O'Kennedy, R.; Lee, H. A.; Morgan, M. R. A., Development of surface plasmon resonance-based immunoassay for aflatoxin B1. J. Agric. Food Chem. 2000, 48, 5097-5104.

30. Meneely, J. P.; Sulyok, M.; Baumgartner, S.; Krska, R.; Elliott, C. T., A rapid optical immunoassay for the screening of T-2 and HT-2 toxin in cereals and maize-based baby food. Talanta 2010, 81, 630-636.

31. Meneely, J. P.; Quinn, J. G.; Flood, E. M.; Hajšlová, J.; Elliott, C. T., Simultaneous screening for T-2/HT-2 and deoxynivalenol in cereals using a surface plasmon resonance immunoassay. World Mycotoxin J. 2012, 5, 117-126.

32. Yuan, J.; Deng, D.; Lauren, D. R.; Aguilar, M.-I.; Wu, Y., Surface plasmon resonance biosensor for the detection of ochratoxin A in cereals and beverages. Anal. Chim. Acta 2009, $656,63-71$.

33. van der Gaag, B.; Spath, S.; Dietrich, H.; Stigter, E.; Boonzaaijer, G.; van Osenbruggen, T.; Koopal, K., Biosensors and multiple mycotoxin analysis. Food Control 2003, 14, 251-254.

34. Giudicatti, S.; Valsesia, A.; Marabelli, F.; Colpo, P.; Rossi, F., Plasmonic resonances in nanostructured gold/polymer surfaces by colloidal lithography. Phys. Status Solidi A 2010, 207, 935-942.

35. Joshi, S.; Pellacani, P.; van Beek, T. A.; Zuilhof, H.; Nielen, M. W. F., Surface characterization and antifouling properties of nanostructured gold chips for imaging surface plasmon resonance biosensing. Sens. Actuators, B 2015, 209, 505-514.

36. Lahiri, J.; Ostuni, E.; Whitesides, G. M., Patterning ligands on reactive SAMs by microcontact printing. Langmuir 1999, 15, 2055-2060.

37. Commission Regulation (EU) No 519/2014 of 16 May 2014 amending Regulation (EC) No $401 / 2006$ as regards methods of sampling of large lots, spices and food supplements, performance criteria for T-2, HT-2 toxin and citrinin and screening methods of analysis. Off. J. Eur. Union 2014, L147, 29-43.

38. Johnsson, B.; Löfås, S.; Lindquist, G., Immobilization of proteins to a carboxymethyldextranmodified gold surface for biospecific interaction analysis in surface plasmon resonance sensors. Anal. Biochem. 1991, 198, 268-277.

39. Löfås, S.; Johnsson, B., A novel hydrogel matrix on gold surfaces in surface plasmon resonance sensors for fast and efficient covalent immobilization of ligands. J. Chem. Soc., Chem. Commun. 1990, 1526-1528.

40. Skládal, P., Effect of methanol on the interaction of monoclonal antibody with free and immobilized atrazine studied using the resonant mirror-based biosensor. Biosens. Bioelectron. 1999, 14, 257-263.

41. Geuijen, K. P. M.; Schasfoort, R. B.; Wijffels, R. H.; Eppink, M. H. M., High-throughput and multiplexed regeneration buffer scouting for affinity-based interactions. Anal. Biochem. 2014, 454, 38-40.

42. Zachariasova, M.; Hajslova, J.; Kostelanska, M.; Poustka, J.; Krplova, A.; Cuhra, P.; Hochel, I., Deoxynivalenol and its conjugates in beer: A critical assessment of data obtained by enzyme-linked immunosorbent assay and liquid chromatography coupled to tandem mass spectrometry. Anal. Chim. Acta 2008, 625, 77-86.

43. Krishnamoorthy, S.; Himmelhaus, M., Confinement-induced enhancement of antigenantibody interactions within binary nanopatterns to achieve higher efficiency of on-chip immunosensors. Adv. Mater. 2008, 20, 2782-2788.

44. Valsesia, A.; Marabelli, F.; Giudicatti, S.; Marchesini, G. R.; Rossi, F.; Colpo, P., SPR sensor device with nanostructure. World Patent 2013, WO 2013/007448 A1. 


\section{Supporting information}

\begin{tabular}{|l|l|l|}
\hline Day 1 & Day 2 & Day 3 \\
\hline B1-1.1 & B1-2.1 & B1-3.1 \\
\hline B1-1.2 & B1-2.2 & B1-3.2 \\
\hline B1-1.3 & B1-2.3 & B1-3.3 \\
\hline B2-1.1 & B2-2.1 & B2-3.1 \\
\hline B2-1.2 & B2-2.2 & B2-3.2 \\
\hline B2-1.3 & B2-2.3 & B2-3.3 \\
\hline B3-1.1 & & \\
\hline B3-1.2 & & \\
\hline B3-1.3 & & \\
\hline
\end{tabular}

Scheme S1. Extraction scheme for three different barley samples (B1, B2, B3) on three different days to obtain 21 blank and 21 spiked samples for validation experiment.
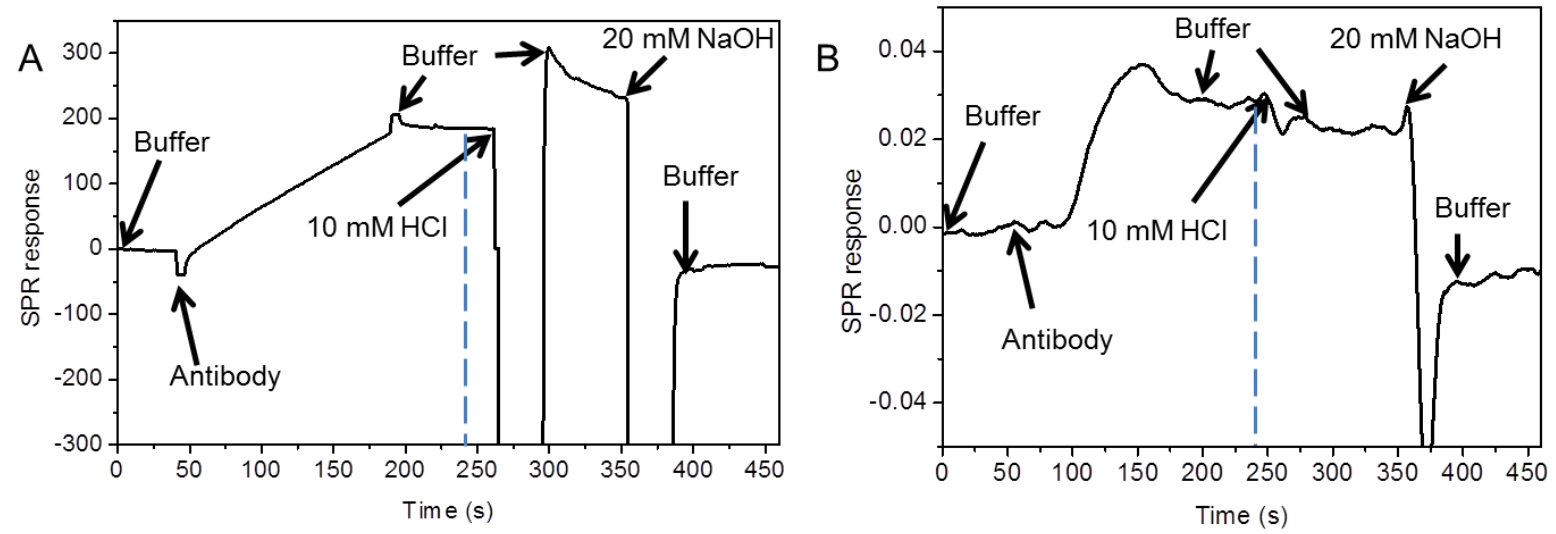

Figure S1. SPR sensorgrams generated in a channel (for SPR) or ROI (for iSPR) with $F B_{1}-O_{V A}$ upon injection of A) $0.1 \mu \mathrm{g} / \mathrm{ml}$ anti-FB ${ }_{1}$ measured using a Biacore 3000 with flat gold and B) 5 $\mu \mathrm{g} / \mathrm{ml}$ anti-FB ${ }_{1}$ measured using a prototype iSPR instrument with nanostructured gold. Each cycle consists of flushing with buffer, injection of antibody and regeneration with $10 \mathrm{mM} \mathrm{HCl}(30 \mathrm{~s})$ followed by $20 \mathrm{mM} \mathrm{NaOH}$ (30 s). After injection of antibody and regeneration, the chip was flushed with buffer. The blue dotted line shows the time point where the SPR response was recorded. 


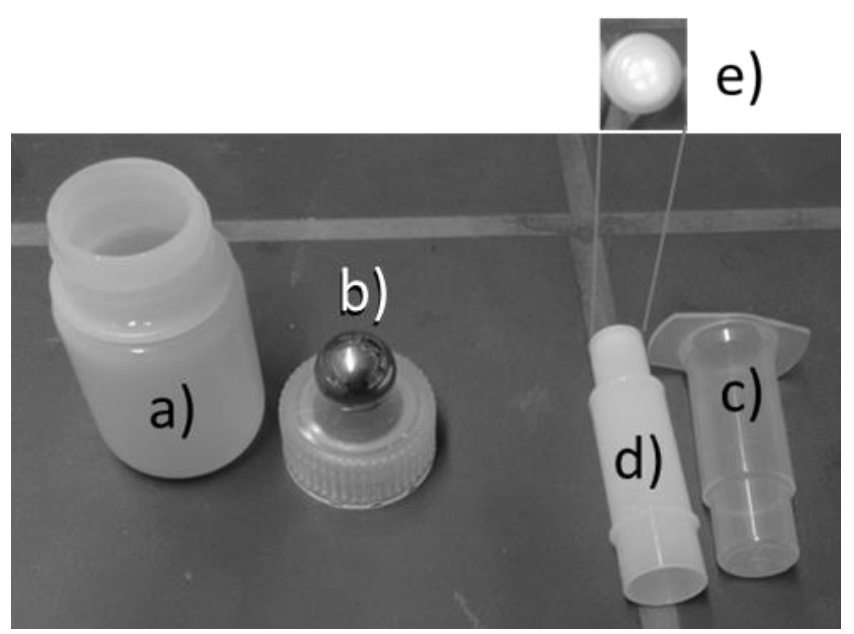

Figure S2. Kit used for simplified sample preparation suitable for field applications. The extraction was done using a plastic container (a) and a stainless steel ball (b). The extract was collected in the upper tube of the filter device (c) and filtered into the plunger (d) through the filter device (e).

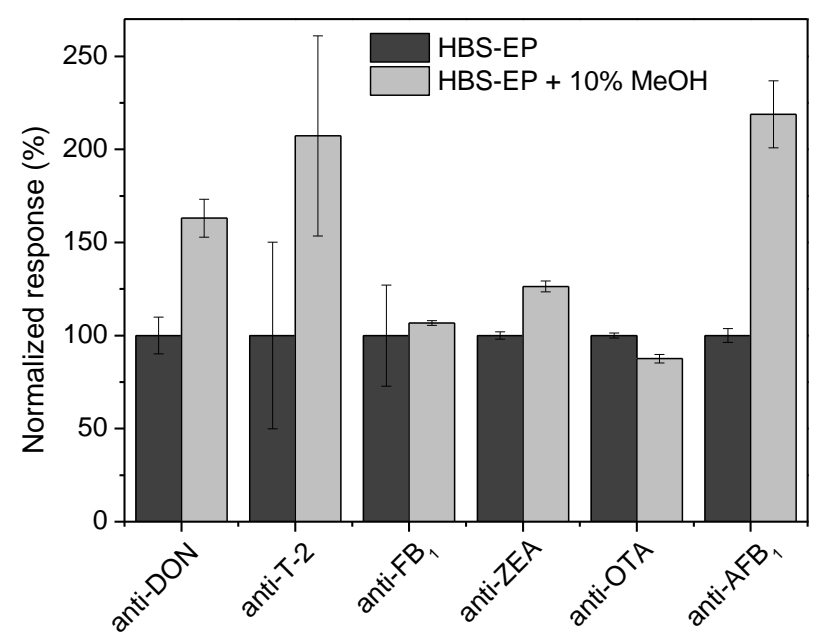

Figure S3. Comparison of binding of the six antibodies with their corresponding OVA conjugates in HBS-EP without and with $10 \% \mathrm{MeOH}$ measured with SPR (Biacore) $(n=3)$. The responses are normalized to the binding of the corresponding antibodies in HBS-EP. 


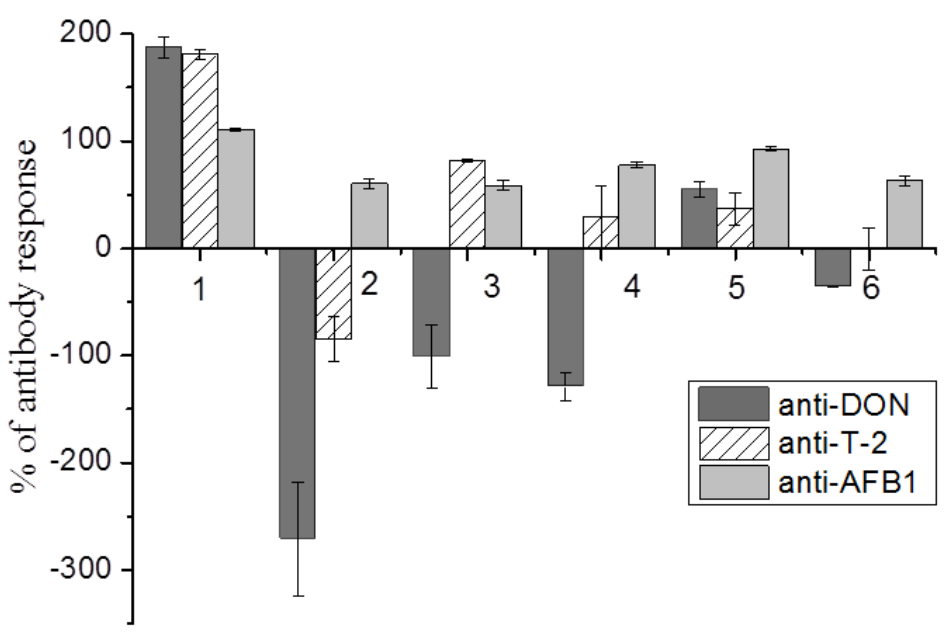

Figure S4. Regeneration scouting for anti-DON, anti-T-2 and anti-AFB ${ }_{1}$ performed using SPR (Biacore). The percentage of antibody response remaining after regeneration is plotted for three different antibodies and six different regeneration conditions $(1=6 \mathrm{M}$ guanidine $\mathrm{HCl}, 2=20 \mathrm{mM}$ $\mathrm{NaOH}, 3=5 \mathrm{mM} \mathrm{NaOH}+0.5 \%$ SDS, $4=5 \mathrm{mM} \mathrm{NaOH}$ followed by $0.1 \%$ Tween20, $5=10 \mathrm{mM} \mathrm{HCl}$ followed by $10 \mathrm{mM} \mathrm{NaOH}$ and $6=10 \mathrm{mM} \mathrm{HCl}$ followed by $20 \mathrm{mM} \mathrm{NaOH}$ ). All regeneration solutions were injected for $30 \mathrm{~s}$ at a flow rate of $100 \mu \mathrm{L} / \mathrm{min}(n=3)$. Please note that the other three antibodies (anti-ZEA, anti-FB ${ }_{1}$ and anti-OTA) are not shown here but were successfully regenerated under regeneration condition 6 .
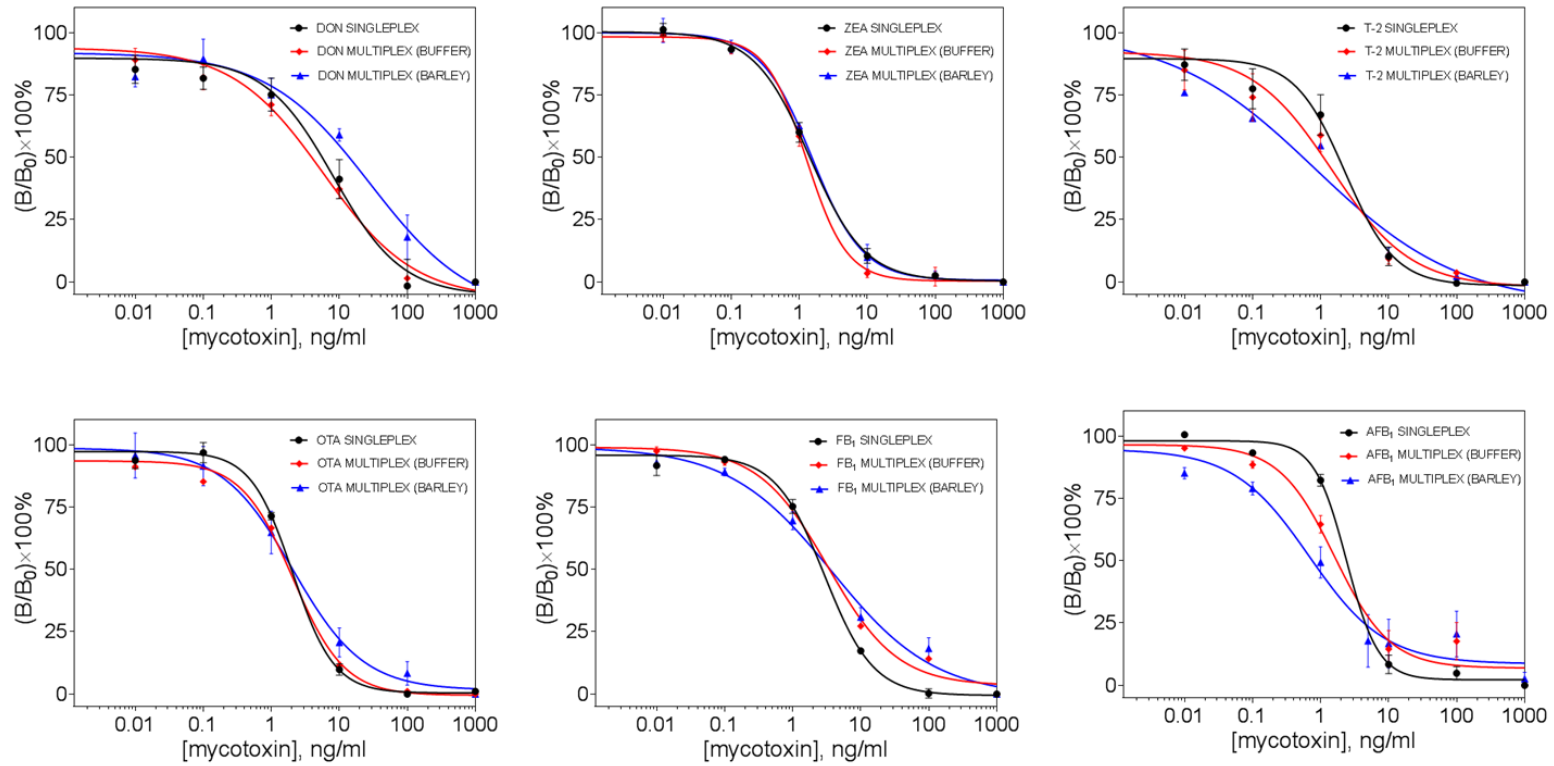

Figure S5. Calibration curves of the double 3-plex assay using SPR (Biacore) for singleplex in buffer, multiplex in buffer and multiplex in barley extract $(n=3)$. Two carboxymethylated chips were used: one for DON, ZEA and T-2 and another for OTA, FB ${ }_{1}$ and $\mathrm{AFB}_{1}$. 

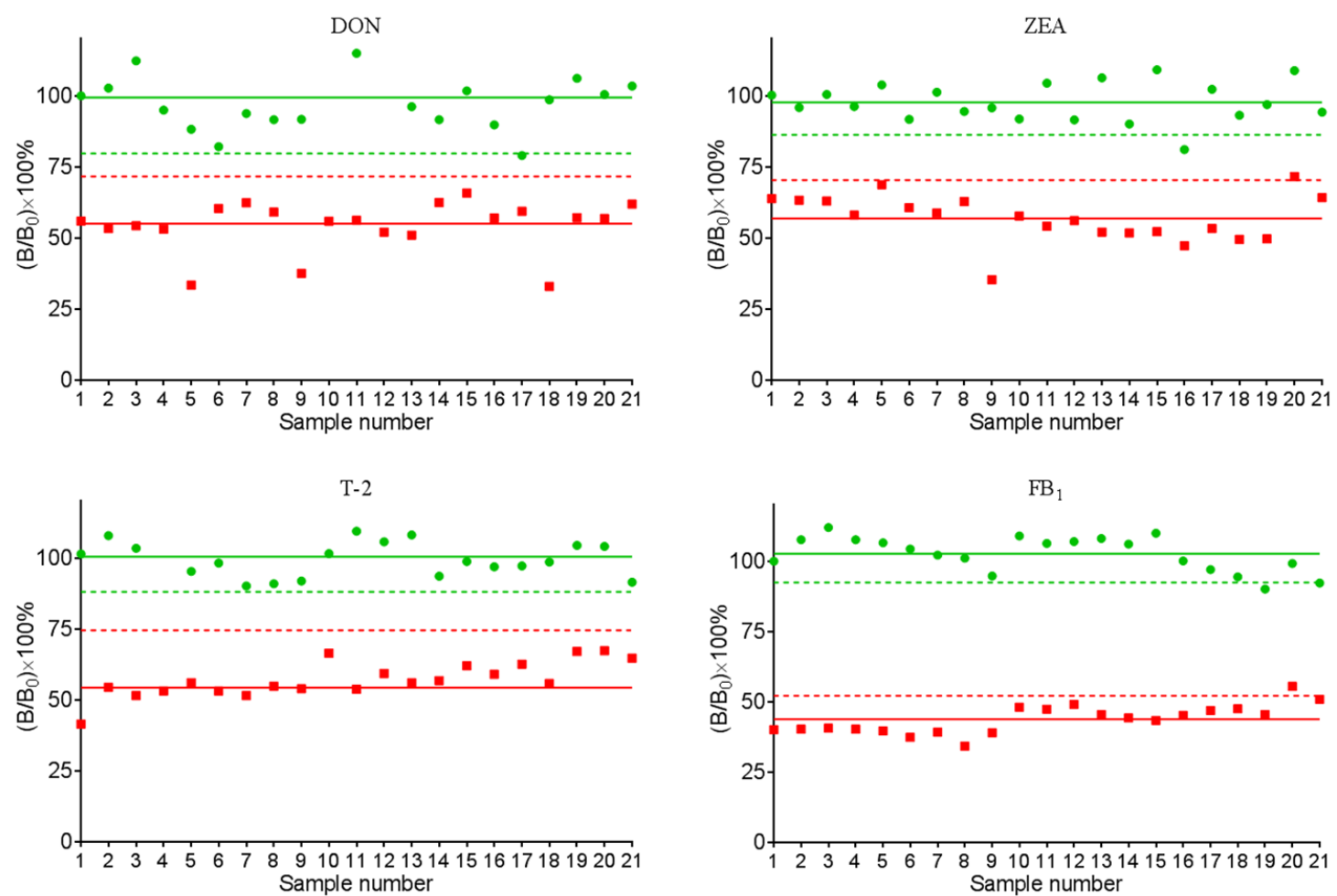

Figure S6. Validation graphs for four mycotoxins (DON, ZEA, T-2 and FB 1 ) in spiked barley extract in the double 3-plex assay (using Biacore). The responses of the blank samples (dots) and spiked samples (squares) are relative to the response of a mixed blank barley sample. The averages of the responses of the blank samples (green solid line) and spiked samples (red solid line), the threshold values (green dotted line) and cut-off factors (red dotted line) are also shown in the graph.
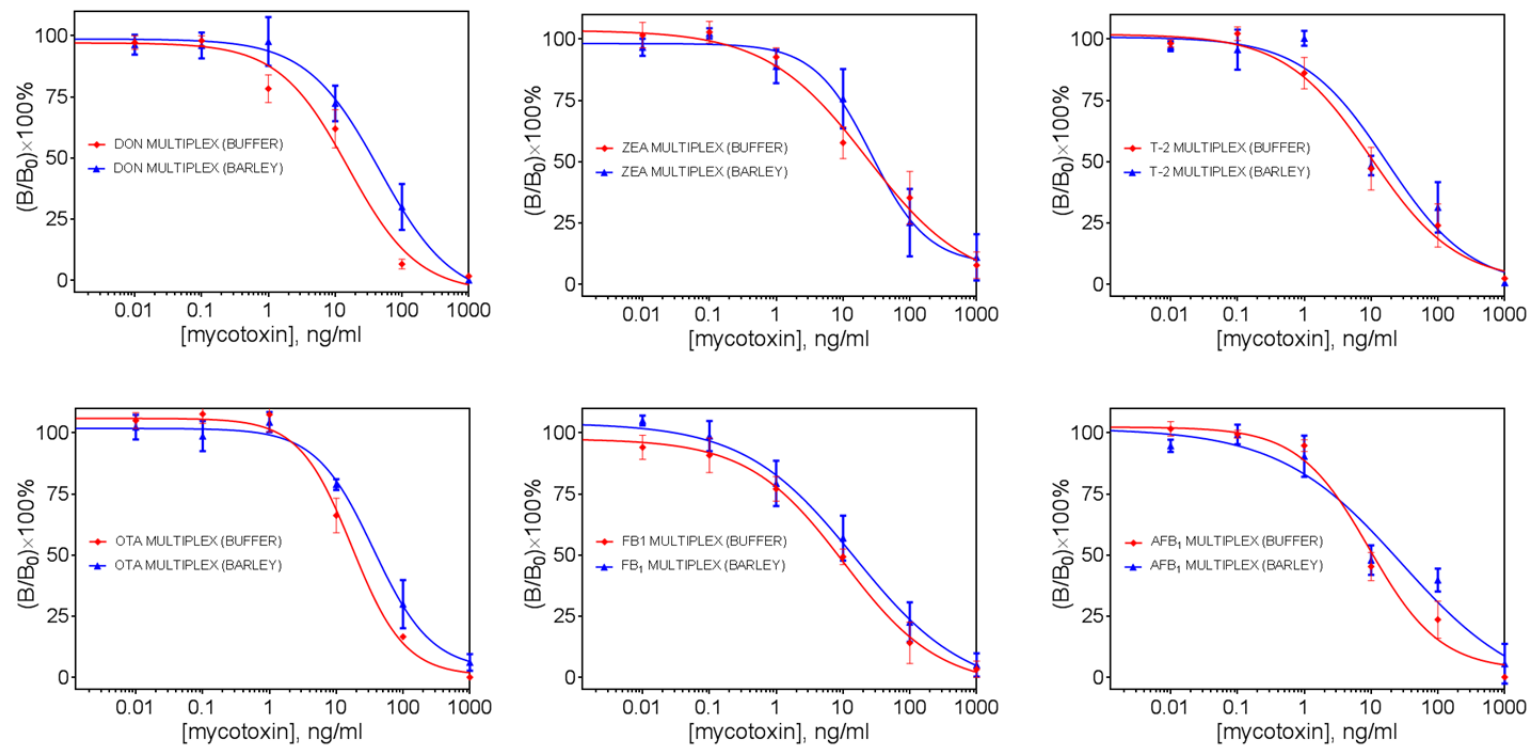

Figure S7. Calibration curves of the 6-plex assay the prototype nanostructured iSPR for multiplex in buffer and multiplex in barley extract $(n=3)$. A single PEG3500 chip was used for detection of DON, ZEA, T-2, OTA, FB 1 and $\mathrm{AFB}_{1}$. 


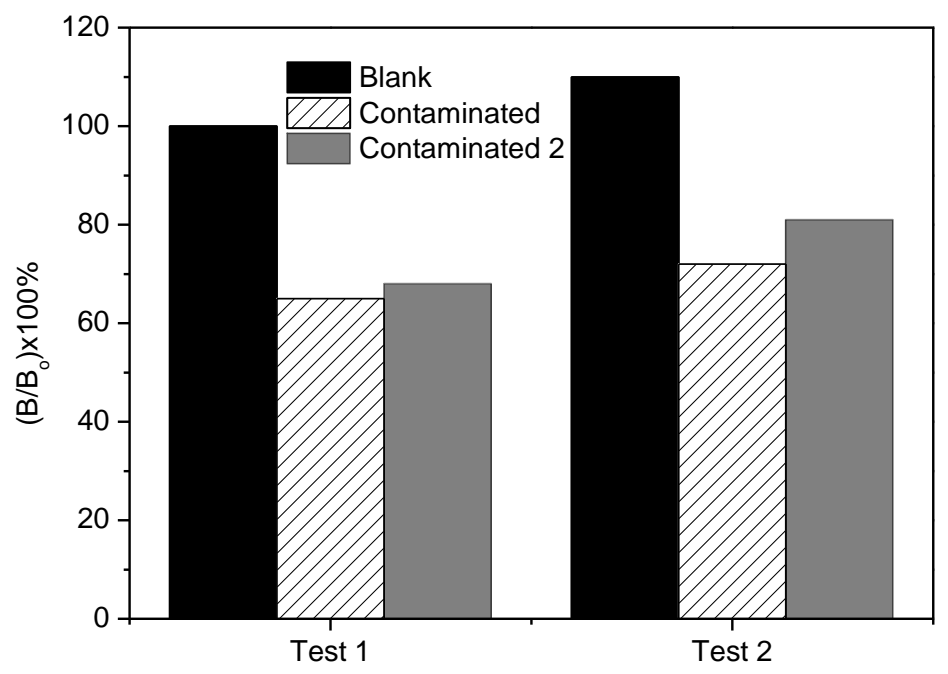

Figure S8. Relative responses measured in duplicate for naturally contaminated barley sample (CS2) for presence of ZEA in a 6-plex format using the prototype nanostructured iSPR instrument. The responses are relative to the response of a mixed blank barley sample. Contaminated sample 1 was extracted using the laboratory based extraction protocol whereas contaminated sample 2 was extracted using a portable plastic extraction device (see experimental section). 
Table S1. Limit of detection (LOD), IC50 and working range of the double-3plex assay (using Biacore) and 6plex (using prototype nanostructured iSPR) for singleplex in buffer, multiplex in buffer and multiplex in barley extract $(n=3)$. All values are expressed in $n g$ of toxin per ml of buffer or barley extract.

\begin{tabular}{|c|c|c|c|c|c|c|c|}
\hline \multirow[b]{2}{*}{ Toxin } & \multirow[b]{2}{*}{ Format } & \multicolumn{3}{|c|}{ Double 3-plex SPR (Biacore) } & \multicolumn{3}{|c|}{ 6-plex nanostructured iSPR } \\
\hline & & $\operatorname{LOD}^{a}$ & IC50 & $\begin{array}{l}\text { Working } \\
\text { range }^{b}\end{array}$ & $\operatorname{LOD}^{a}$ & IC50 & $\begin{array}{l}\text { Working } \\
\text { range }^{b}\end{array}$ \\
\hline \multirow[t]{3}{*}{ DON } & Singleplex & $0.6^{*}$ & 5.6 & $0.6-25$ & - & - & - \\
\hline & $\begin{array}{l}\text { Multiplex } \\
\text { (buffer) }\end{array}$ & $0.3^{*}$ & 3.9 & $0.3-25$ & 0.6 & 12 & $2-60$ \\
\hline & $\begin{array}{l}\text { Multiplex } \\
\text { (barley) }\end{array}$ & $0.8^{*}$ & 15 & $0.8-100$ & 2 & 38 & $6-150$ \\
\hline \multirow[t]{3}{*}{ ZEA } & Singleplex & 0.2 & 1.5 & $0.5-5$ & - & - & - \\
\hline & $\begin{array}{l}\text { Multiplex } \\
\text { (buffer) }\end{array}$ & 0.2 & 1.3 & $0.5-3$ & 0.8 & 25 & $3-150$ \\
\hline & $\begin{array}{l}\text { Multiplex } \\
\text { (barley) }\end{array}$ & 0.2 & 1.6 & $0.5-5$ & 3 & 30 & $7-250$ \\
\hline \multirow[t]{3}{*}{$\mathrm{T}-2$} & Singleplex & $0.4^{*}$ & 1.9 & $0.4-6$ & - & - & - \\
\hline & $\begin{array}{l}\text { Multiplex } \\
\text { (buffer) }\end{array}$ & $0.1^{*}$ & 1.2 & $0.1-7$ & 0.5 & 10 & $1.5-80$ \\
\hline & $\begin{array}{l}\text { Multiplex } \\
\text { (barley) }\end{array}$ & $0.02 *$ & 0.5 & $0.02-9$ & 0.8 & 18 & $2.5-120$ \\
\hline \multirow[t]{3}{*}{ OTA } & Singleplex & 0.4 & 2.0 & $0.8-5$ & - & - & - \\
\hline & $\begin{array}{l}\text { Multiplex } \\
\text { (buffer) }\end{array}$ & 0.1 & 1.8 & $0.4-6$ & 3.8 & 20 & $6-70$ \\
\hline & $\begin{array}{l}\text { Multiplex } \\
\text { (barley) }\end{array}$ & 0.1 & 2.1 & $0.4-10$ & 5 & 40 & $10-180$ \\
\hline \multirow[t]{3}{*}{$\mathrm{FB}_{1}$} & Singleplex & 0.3 & 2.7 & $0.8-9$ & - & - & - \\
\hline & $\begin{array}{l}\text { Multiplex } \\
\text { (buffer) }\end{array}$ & 0.2 & 3.5 & $0.6-20$ & 0.2 & 8 & $0.8-70$ \\
\hline & $\begin{array}{l}\text { Multiplex } \\
\text { (barley) }\end{array}$ & 0.07 & 3.5 & $0.3-40$ & 0.4 & 15 & $1.5-120$ \\
\hline \multirow[t]{3}{*}{$\mathrm{AFB}_{1}$} & Singleplex & 0.7 & 2.5 & $1-5$ & - & - & - \\
\hline & $\begin{array}{l}\text { Multiplex } \\
\text { (buffer) }\end{array}$ & 0.2 & 1.7 & $0.4-8$ & 0.8 & 10 & $2-60$ \\
\hline & $\begin{array}{l}\text { Multiplex } \\
\text { (barley) }\end{array}$ & 0.02 & 0.8 & $0.1-8$ & 0.3 & 20 & $1.5-250$ \\
\hline
\end{tabular}

a LOD is defined as IC10 (concentration at which $10 \%$ inhibition of binding occurs) unless marked with an asterisk

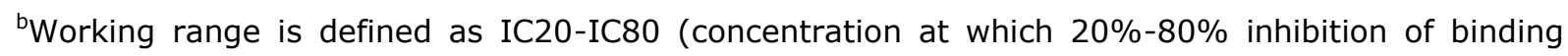
occurs)

*IC20 is used as LOD, as the fitting does not allow determination of IC10 for this toxin 



\section{Chapter 4}

\section{Analysis of mycotoxins in beer using a portable nanostructured imaging surface plasmon resonance biosensor}

Sweccha Joshi, ${ }^{1,2}$, Rumaisha M. Annida ${ }^{1}$, Han Zuilhof ${ }^{1}$, Teris A. van Beek ${ }^{1}$, Michel W.F. Nielen ${ }^{1,3}$

${ }^{1}$ Laboratory of Organic Chemistry, Wageningen University, Stippeneng 4, 6708 WE Wageningen, The Netherlands

${ }^{2}$ TI-COAST, Science Park 904, 1098 XH Amsterdam, The Netherlands

${ }^{3}$ RIKILT Wageningen University \& Research, P.O. Box 230, 6700 AE Wageningen, The Netherlands

This chapter has been published in:

Journal of Agricultural and Food Chemistry, 2016, 64, 8263-8271.

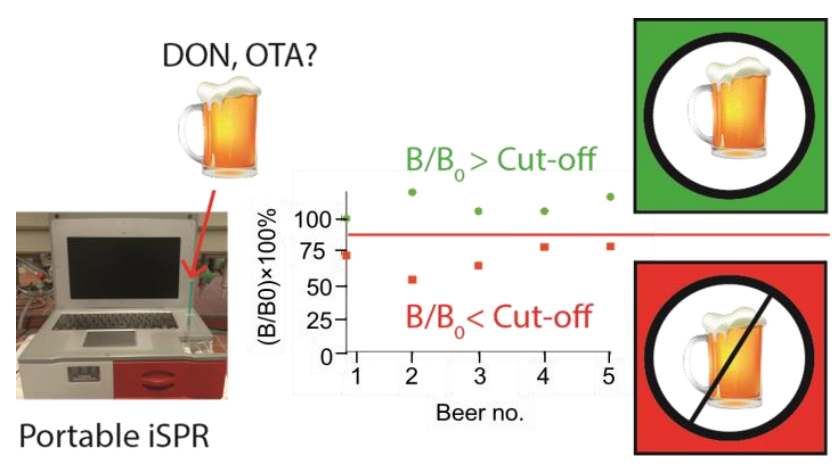




\section{Abstract}

A competitive inhibition immunoassay is described for the mycotoxins deoxynivalenol (DON) and ochratoxin A (OTA) in beer using a portable nanostructured imaging surface plasmon resonance (iSPR) biosensor, also referred to as imaging nanoplasmonics. The toxins were directly and covalently immobilized on a 3-dimensional carboxymethylated dextran (CMD) layer on a nanostructured iSPR chip. The assay is based on competition between the immobilized mycotoxins and free mycotoxins in the solution for binding to specific antibodies. The chip surface was regenerated after each cycle and the combination of CMD and direct immobilization of toxins allowed the chips to be used for more than 450 cycles. The limits of detection (LODs) in beer were $17 \mathrm{ng} / \mathrm{mL}$ for DON and $7 \mathrm{ng} / \mathrm{mL}$ for OTA (or $0.09 \mathrm{ng} / \mathrm{mL}$ after 75 times enrichment). These LODs allowed detection of even less than $10 \%$ depletion of the tolerable daily intake of DON and OTA by beer. Significant cross-reactivity of anti-DON was observed towards DON-3-glucoside and 3-acetyl-DON while no cross-reactivity was seen for 15-acetyl-DON. A preliminary inhouse validation with 20 different batches of beer showed that both toxins can be detected at the considered theoretical safe level for beer. The assay can be used for infield or at-line detection of DON in beer and also in barley without pre-concentration, while OTA in beer requires an additional enrichment step thus making the latter in its present form less suitable for field applications.

\section{Keywords}

Imaging SPR, mycotoxins, beer, nanoplasmonics, deoxynivalenol, ochratoxin A 


\section{Introduction}

Mycotoxins are the secondary metabolites of fungi commonly found in several foods, beverages, and animal feed and are known to be teratogenic, mutagenic, and carcinogenic. ${ }^{1}$ They are carried-over from infected barley into malt and ultimately to beer due to their thermal stability and relatively good water solubility. ${ }^{2-4}$ Therefore, careful screening of beer ingredients and end products is required for safety of the consumers. The occurrence of different mycotoxins in beer has been reported earlier with most studies focusing on deoxynivalenol (1; DON) mainly due to its high incidence. ${ }^{5,6}$ Another mycotoxin, ochratoxin A (5; OTA), although detected in beer at low concentrations (only $<0.2 \mathrm{ng} / \mathrm{mL}$ ), ${ }^{7,8}$ is of interest due to its high toxicity. According to EU legislation, the maximum levels (MLs) relevant for barley are set to be $1250 \mu \mathrm{g} / \mathrm{kg}$ for DON (in unprocessed cereals other than durum wheat, oats and maize) and $5 \mu \mathrm{g} / \mathrm{kg}$ for OTA (in unprocessed cereals). ${ }^{9}$ At present there is no specific regulation for mycotoxins in beer; only a tolerable daily intake (TDI) of $1 \mu \mathrm{g} \mathrm{DON}{ }^{10}$ and a tolerable weekly intake (TWI) of $120 \mathrm{ng} \mathrm{OTA}^{11}$ per $\mathrm{kg}$ body weight are defined. For a person with $70 \mathrm{~kg}$ body weight and a beer consumption of $300 \mathrm{~mL}$, the maximum allowable concentrations in beers based on the TDIs are $233 \mathrm{ng} / \mathrm{mL}$ for DON and $4 \mathrm{ng} / \mathrm{mL}$ for OTA. Measuring $10 \%$ of the TDI depletion would require sensitivities of $23 \mathrm{ng} / \mathrm{mL}$ and $0.4 \mathrm{ng} / \mathrm{mL}$ for DON and OTA respectively. Solely for in-house validation purposes, we defined 'theoretical safe limits' (TSLs) and set them at $120 \mathrm{ng} / \mathrm{mL}$ for DON and $0.2 \mathrm{ng} / \mathrm{mL}$ for OTA in beer. They correspond to $50 \%$ TDI depletion for DON and only 5\% TDI depletion for the more toxic OTA.

In recent years, several methods have been developed for the detection of mycotoxins in different food and beverages. ${ }^{12-15}$ Literature about different methods for detection of mycotoxins in cereals was covered in our previous publication. ${ }^{16}$ Most methods for the detection of DON and OTA in beer are based on liquid chromatography (LC) coupled with mass spectrometric (MS) detection. ${ }^{2,6,17-21}$ Although LC-MS based methods are sensitive and can be used for quantitative analysis of the mycotoxins, they are quite expensive, laboratory based, and require expert personnel. Another laboratory based method for the detection of mycotoxins in beer is gas chromatography coupled with mass spectrometry (GC-MS) with similar demerits as LC-MS. ${ }^{22}$ Immunological assay kits $^{23}$ offer a simple, portable, semi-quantitative solution for mycotoxin detection with enzyme-linked immunosorbent assay (ELISA) being the most commonly used method. ${ }^{17,24-26}$ Amongst the immunological methods, surface plasmon resonance (SPR) biosensing has been used for the detection of single or multiple mycotoxins in different cereals including the malt ingredient barley. ${ }^{16,27}$ However, no SPR assay has been 
described for the detection of mycotoxins in beer, let alone in a portable and nanostructured format.

The aim of this study was, therefore, to develop an assay for the detection of two relevant mycotoxins in beer, namely DON and OTA (Figure 1), using a novel portable imaging SPR (iSPR) instrument. A preliminary in-house validation is performed for both toxins in a range of beers including measurement of naturally contaminated beer samples. The nanostructured iSPR instrument is portable thus making it interesting for in-field and at-line detection of mycotoxins in beer and in beer production chains. The latter is supported by at-line application of the beer assay for DON inside a brewery and by preliminary results for testing of barley samples.

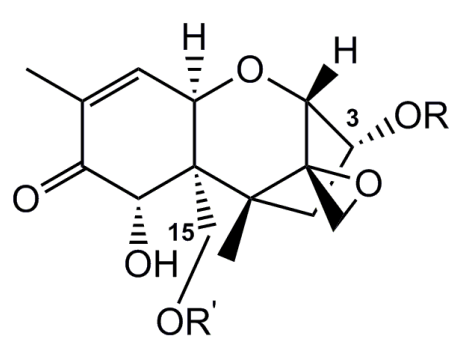

$$
\begin{aligned}
& R=H, R^{\prime}=H \\
& \begin{array}{l}
\mathbf{1} \\
R=-(C=O)-C H_{3}, R^{\prime}=H \\
\\
2
\end{array} \\
& R=H, R^{\prime}=-(C=O)-C H_{3}
\end{aligned}
$$$$
3
$$<smiles>C[C@H]1Cc2c(Cl)cc(C(=O)N[C@@H](Cc3ccccc3)C(=O)O)c(O)c2C(=O)O1</smiles>

5

$R=\beta$-D-glucoside,$R^{\prime}=H$

4

Figure 1. Chemical structures of deoxynivalenol (DON, 1), 3-acetyl DON (3ADON, 2), 15-acetyl DON (15ADON, 3), deoxynivalenol-3-glucoside (D3G, 4) and ochratoxin A (OTA, 5).

\section{Materials and methods}

\section{Biosensor chips}

Nanostructured gold chips were produced by Plasmore Srl. (Ispra, Italy) using colloidal lithography and plasma-enhanced chemical vapor deposition (PE-CVD). ${ }^{28}$ Briefly, poly(methyl methacrylate) (PMMA) was deposited on the glass substrate by PE-CVD using methyl methacrylate as liquid precursor. Spin coating was then used to cover the film with polystyrene (PS) beads (500 nm with a nominal size dispersion of $10 \%$ ). Next, the sample was exposed to oxygen plasma etching to remove the PMMA from the areas not covered by the beads and to reduce the size of the PS beads to provide the required periodicity. A gold layer of 100-200 nm was then deposited on the surface by physical vapor deposition. Finally, the polystyrene beads were removed, resulting in a surface with a gold film perforated by PMMA. The combined control of deposition and etching parameters allowed fine tuning of film thickness and well diameters. The PMMA regions, with diameters of 200-250 $\mathrm{nm}$ and periodicity (distance between the centers of the two 
PMMA wells) of $500-600 \mathrm{~nm}$, make up around $20 \%$ of the total surface area (Figure $2 \mathrm{~A}$ ). The nanostructured gold acting as a nanograting allows the omission of prisms in the optical setup of the instrument (Figure 2B). Detailed characterization of the nanostructured iSPR chips can be found in an earlier publication. ${ }^{29}$ The nanostructured gold chips were further modified with carboxymethylated dextran of $200 \mathrm{~nm}$ thickness with a high density of carboxymethylated groups (CMD200D) by Xantec (Düsseldorf, Germany).

\section{Chemicals}

Ammonia, anhydrous dimethyl sulfoxide (DMSO), boric acid, 1,1'-carbonyldiimidazole (CDI), ethanolamine hydrochloride, 1-ethyl-3-(3-dimethylaminopropyl) carbodiimide hydrochloride (EDC), formic acid, hydrochloric acid, 2,2'(ethylenedioxy)bis(ethylamine) (Jeffamine), $N$-hydroxysuccinimide (NHS), dibasic potassium phosphate, monobasic potassium phosphate, sodium bicarbonate, sodium carbonate, sodium hydroxide and sodium tetraborate (Sigma Aldrich, Zwijndrecht, The Netherlands), methanol and acetonitrile (VWR, Amsterdam, The Netherlands) were used as obtained. HBS-EP (0.01 M HEPES pH 7.4, $0.15 \mathrm{M} \mathrm{NaCl}, 3 \mathrm{mM}$ EDTA, 0.005\% v/v Surfactant P20) (GE Healthcare, Diegem, Belgium) was used as buffer. MilliQ water $(18.3 \mathrm{M} \Omega \cdot \mathrm{cm}$ resistivity) was obtained using a water purification system (Merck, Amsterdam, The Netherlands). Oasis MAX mixed anion exchange SPE columns (6 cc Vac Cartridge, 150 mg sorbent per cartridge, $60 \mu \mathrm{m}$ particle size) (Waters, Etten-Leur, The Netherlands) were used for enrichment of OTA.

\section{Antibodies and toxins}

Monoclonal antibodies (mAbs) against DON (AB-02-02) (Aokin AG, Berlin, Germany) and mAbs against OTA (SFH-OTA-005) (Soft Flow Biotechnology, Pecs, Hungary) were used for all experiments. Solid mycotoxin and reference solutions of DON 1, 3-acetyl-DON (3ADON) 2, 15-acetyl-DON (15ADON) 3, deoxynivalenol-3-glucoside (D3G) 4, and OTA 5 (Biopure via RomerLabs, Tulln, Austria) were used (Figure 1).

\section{Samples}

Nine pale lager beers (five with $5 \%$ alcohol, two pilsners with $5 \%$ alcohol, one Czech pilsner with $4.4 \%$ alcohol, one alcohol free), one dark ale beer (abbey dubbel with $6.8 \%$ alcohol), and one strong pale ale beer (abbey tripel with $9.5 \%$ alcohol) were bought from Dutch supermarkets. Twenty different batches of one of the pale lager beers were selected for the validation. Contaminated beer 1 (CB1) containing $2760 \pm 95 \mathrm{ng} / \mathrm{mL}$ of DON and $3883 \pm 137 \mathrm{ng} / \mathrm{mL}$ of D3G according to previous literature (measured using LC-MS/MS $)^{19}$ was kindly provided by Dr. Milena Zachariasova. Two other contaminated 
beers, respectively containing $140 \mathrm{ng} / \mathrm{mL}$ of DON (CB2) and $182 \mathrm{ng} / \mathrm{ml}$ of DON plus 43 $\mathrm{ng} / \mathrm{ml}$ D3G (CB3) were kindly provided by Jeroen Peters. The LC-MS/MS measurements were done using an in-house validated quantitative method developed at RIKILT (Wageningen, the Netherlands), which is ISO 17025 accredited for feed. Blank barley containing DON below the limit of detection according to ELISA ( $0.1 \mathrm{ppm})$ was obtained from project partners.

\section{Immobilization of mycotoxins}

Immobilization of DON and OTA onto carboxymethylated dextran was based on literature protocols ${ }^{30,31}$ with slight modifications as described below. The entire protocol was performed at room temperature.

DON 1 (Figure 1) was activated using the hydroxyl group by dissolving $1 \mathrm{mg}$ of the toxin and $2.5 \mathrm{mg}$ of CDI in $100 \mu \mathrm{L}$ of anhydrous DMSO inside a glovebox (Argon 6.0, $\mathrm{O}_{2}<0.1 \mathrm{ppm}$, and $\mathrm{H}_{2} \mathrm{O}<0.1 \mathrm{ppm}$ ) and the reaction mixture was left for $1 \mathrm{~h}$. The activated toxin was then transferred out of the glovebox and mixed $(1: 1, \mathrm{v} / \mathrm{v})$ with 50 mM sodium carbonate buffer ( $\mathrm{pH}$ 9.0). A solution of OTA was obtained by dissolving $1 \mathrm{mg}$ of the toxin in $200 \mu \mathrm{L}$ of acetonitrile:water $(80: 20 \mathrm{v} / \mathrm{v})$ and $200 \mu \mathrm{L}$ of $50 \mathrm{mM}$ sodium carbonate buffer ( $\mathrm{pH}$ 9.0). The carboxylic acid group of OTA 5 (Figure 1) was activated by mixing $20 \mu \mathrm{L}$ of the OTA solution with $20 \mu \mathrm{L}$ of $0.4 \mathrm{M}$ EDC and $20 \mu \mathrm{L}$ of $0.1 \mathrm{M}$ NHS, and incubating for $45 \mathrm{~min}$. Stocks of both toxin solutions (DON in DMSO and OTA in 80\% acetonitrile plus carbonate buffer) were divided into aliquots of $20 \mu \mathrm{L}$ and stored at -20 ${ }^{\circ} \mathrm{C}$ and one aliquot was used for activation and immobilization.

The immobilization was performed manually on the bench. The carboxymethylated dextran modified chip was washed with MilliQ water and dried with nitrogen after each step. The entire chip was activated with $50 \mu \mathrm{L}$ of a $1: 1(\mathrm{v} / \mathrm{v})$ mixture of $0.4 \mathrm{M}$ EDC and $0.1 \mathrm{M}$ NHS for $30 \mathrm{~min}$. To the activated chip, $50 \mu \mathrm{L}$ of Jeffamine $(0.1 \mathrm{M}$ in $50 \mathrm{mM}$ borate buffer $\mathrm{pH}$ 8.5) was added to obtain an amine terminated dextran chip. After $1 \mathrm{~h}$, the unreacted sites were blocked using $1 \mathrm{M}$ ethanolamine, $\mathrm{pH} 8.5$ for $30 \mathrm{~min}$. Finally, $0.5 \mu \mathrm{L}$ of each of the activated toxin solution was spotted on different areas of the chip. After repeating the spotting five times, the chip was left on the bench for $1 \mathrm{~h}$. The spotted chips were dried with nitrogen and stored at $4-8{ }^{\circ} \mathrm{C}$ until use. Following the immobilization of the toxins and before performing the SPR experiments, the surface was stabilized using 2-3 regeneration cycles with $20 \mathrm{mM} \mathrm{NaOH}$ to obtain a stable baseline.

\section{iSPR measurement}

iSPR measurements were performed using a iSPR instrument, also referred to as imaging nanoplasmonics (Plasmore Srl.). ${ }^{32,33}$ Regions of interest (ROIs) (Figure 2C) were defined where the spots of DON and OTA were present. Two reference ROIs were chosen, one 
outside the flow cell ( ref $_{\text {out, }}$ to correct for any instrumental variations) and the other inside the flow cell but not containing any toxins (ref $_{\text {in, }}$ to correct for any fluidic fluctuations and for non-specific binding). A typical sensorgram generated by the instrument displays the time dependent change in intensity of the reflected light as iSPR response. The iSPR response at each time point is relative to the first image detection

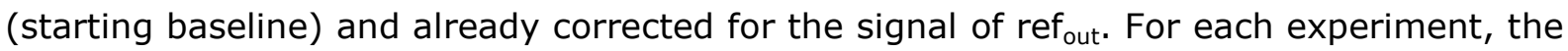
chip was flushed for $2 \mathrm{~min}(\mathrm{t}=0-120 \mathrm{~s}$ ) with running buffer (HBS-EP). The sample (buffer or beer or barley extract) was mixed with the antibodies and was injected at a constant flow rate of $20 \mu \mathrm{L} / \mathrm{min}$ for $5 \mathrm{~min}(t=120-420 \mathrm{~s}$ ). The chip was then flushed with running buffer (HBS-EP) for $4 \mathrm{~min}(\mathrm{t}=420-660 \mathrm{~s})$. The average response during the buffer flow ( $t=500-600 \mathrm{~s}$ ) relative to the starting baseline and after subtracting the response for ref $_{\text {in }}$ was used as the iSPR response in any further calculations. The chip was regenerated by injecting $10 \mathrm{mM} \mathrm{HCl}(100 \mu \mathrm{L} / \mathrm{min})$ for $30 \mathrm{~s}(\mathrm{t}=660-690 \mathrm{~s})$ followed by $20 \mathrm{mM} \mathrm{NaOH}(100 \mu \mathrm{L} / \mathrm{min})$ for $30 \mathrm{~s}(t=750-780 \mathrm{~s})$. The regeneration was followed by washing with HBS-EP buffer ( $t=780-900 \mathrm{~s}$ ) to remove any remaining regeneration solution. The entire cycle took approximately $15 \mathrm{~min}$. The sensorgrams were processed using Excel 2010 and plotted using Origin 8.5. Sensorgrams were smoothened using the Savitzky-Golay function in Origin 8.5 (30 points of window, polynomial order 2 ).

\section{Calibration curves, limit of detection and cross-reactivity}

A solution of $20 \mu \mathrm{g} / \mathrm{mL}$ of anti-DON and/or anti-OTA in HBS-EP containing $20 \%$ methanol was mixed with an equal volume of standard solution or beer or barley extract containing DON and/or OTA (0-10000 ng/mL) for calibration curve in buffer, beer and barley extract, respectively. The iSPR response obtained for the solution containing both toxins and antibodies (B) relative to the maximum response obtained for the solution containing antibodies only $\left(B_{0}\right)$ was used to calculate the relative response as $\left(B / B_{0}\right) \times 100 \%$. The relative response was plotted against the concentration of the toxin to obtain the calibration curves. The calibration curves were fitted in GraphPad Prism (Version 6.0, GraphPad Software Inc., La Jolla, CA) using a non-linear four-parameter model to obtain the IC50 values (concentration at which $50 \%$ inhibition of binding occurs). The IC10 values were used as a measure of the limit of detection (LOD). Additionally, the IC80 and IC20 values (concentrations at which $80 \%$ and $20 \%$ inhibition of binding occurs, respectively) were calculated to determine the working range of the assay. The crossreactivity of the anti-DON for the conjugates (D3G 4, 3ADON 2 and 15ADON 3) was calculated as ( $\left.\mathrm{IC} 50_{\mathrm{DON}} / \mathrm{IC} \mathrm{Conjugate}\right) \times 100 \%$. 


\section{Sample preparation protocol for beer}

All beer samples were degassed for $10 \mathrm{~min}$ in a sonication bath. Optionally, for field use the beer can be degassed by vigorous shaking and venting in between. For DON, $180 \mu \mathrm{L}$ of beer (blank or spiked beer) was mixed with $2 \mu \mathrm{L}$ of anti-DON (1000 $\mu \mathrm{g} / \mathrm{mL}$ in HBS-EP) and $18 \mu \mathrm{L}$ of methanol to obtain a final concentration of $10 \mu \mathrm{g} / \mathrm{mL}$ anti-DON and 108 $\mathrm{ng} / \mathrm{mL}$ DON (for spiked beer) in HBS-EP containing $9 \%$ methanol. For OTA, sample enrichment was done using a mixed anion exchange SPE column. The standard protocol from the manufacturer was optimized using LC with UV absorbance detection (Agilent Technologies, Santa Clara, CA). Based on the UV peak at $332 \mathrm{~nm}$, more than 95\% recovery was obtained using the protocol, which was eventually used in the SPR assay. Briefly, the column was conditioned with $3 \mathrm{~mL}$ of methanol followed by $3 \mathrm{~mL}$ of water. $320 \mu \mathrm{L}$ of $1 \mathrm{M}$ phosphate buffer $(\mathrm{pH} 8)$ was added to $16 \mathrm{~mL}$ of sample (blank or spiked at $0.2 \mathrm{ng} / \mathrm{mL}$ of OTA) to obtain a final $\mathrm{pH}$ value around 6.8 . The beer (15 mL) was then loaded onto the column. Next, the column was rinsed with $5 \mathrm{~mL}$ of $5 \%$ ammonia in water followed by $5 \mathrm{~mL}$ of $100 \%$ methanol. Finally, the beer was eluted with $1 \mathrm{~mL}$ of $2 \%$ formic acid in methanol. The eluate was evaporated completely using a vacuum evaporator and re-dissolved in $100 \mu \mathrm{L}$ of HBS-EP containing $20 \%$ methanol to obtain a beer extract containing a 150 times higher concentration of OTA (30 ng/mL in spiked beer). The enriched beer extract was then mixed $1: 1(\mathrm{v} / \mathrm{v}$ ) with $20 \mu \mathrm{g} / \mathrm{mL}$ of anti-OTA (in HBS-EP) to obtain a final concentration of $10 \mu \mathrm{g} / \mathrm{mL}$ anti-OTA and $15 \mathrm{ng} / \mathrm{mL}$ OTA (for spiked beer) in HBS-EP containing $10 \%$ methanol (75 times enrichment).

\section{Extraction protocol for barley}

$0.5 \mathrm{~g}$ barley was weighed in a $10 \mathrm{~mL}$ centrifuge tube. For spiked barley, $31.3 \mu \mathrm{L}$ of 20 $\mu \mathrm{g} / \mathrm{mL}$ DON in methanol was added to the $0.5 \mathrm{~g}$ in order to get a spiking level at the maximum level $(\mathrm{ML})(1250 \mu \mathrm{g} / \mathrm{kg})$. The tube was then shaken to mix the toxin with the barley and allowed to stand on the bench for $5 \mathrm{~min}$ to allow the solvent to evaporate. Extraction of blank and spiked barley was performed by adding $2 \mathrm{~mL}$ of HBS-EP containing $20 \%$ methanol, followed by vigorous shaking of the mixture (manually) for 5 min. The mixture was then allowed to settle on the bench for $5 \mathrm{~min}$. $700 \mu \mathrm{L}$ of the supernatant was collected and filtered using a $32 \mathrm{~mm}$ syringe filter having a $0.2 \mu \mathrm{m}$ Supor membrane (Pall Life Sciences, Port Washington, NY). Subsequently, $300 \mu \mathrm{L}$ of the filtered barley extract was mixed $1: 1$ with anti-DON (20 $\mu \mathrm{g} / \mathrm{mL}$ in HBS-EP). In case of spiked barley, considering full recovery, the final concentration of DON is $156 \mathrm{ng} / \mathrm{mL}$ prior to iSPR analysis. 


\section{Preliminary in-house validation}

In-house validation was performed using 20 blank and 20 spiked pale lager beer samples. These samples (blank and spiked beer) were composed of different batch numbers of beer from a single brand in different packaging formats. The beer samples were spiked at the set theoretical safe limit (TSL), i.e., $120 \mathrm{ng} / \mathrm{mL}$ for DON and 0.2 $\mathrm{ng} / \mathrm{mL}$ for OTA. Data evaluation was based on EU criteria for the validation of screening methods. ${ }^{34}$ Validation of a semi-quantitative screening method requires the determination of a cut-off level $(\mathrm{Fm})$ and a threshold value $(\mathrm{T})$. When the relative response $\left(B / B_{0}\right)$ is at or below the cut-off level, the sample is categorized as 'screen positive' and liable to confirmatory analysis. As described in Annex II of the guidelines, ${ }^{34}$ the threshold value $(T)$ is defined as:

$$
\mathrm{T}=\mathrm{B}-1.729 \times \mathrm{SDb}
$$

where $B$ is the average response of the blank samples and SDb is the standard deviation of these responses. The cut-off factor $(\mathrm{Fm})$ is defined as:

$$
\mathrm{Fm}=\mathrm{M}+1.729 \times \mathrm{SD}
$$

where $M$ is the average responses of spiked samples and SD is the standard deviation of these responses.

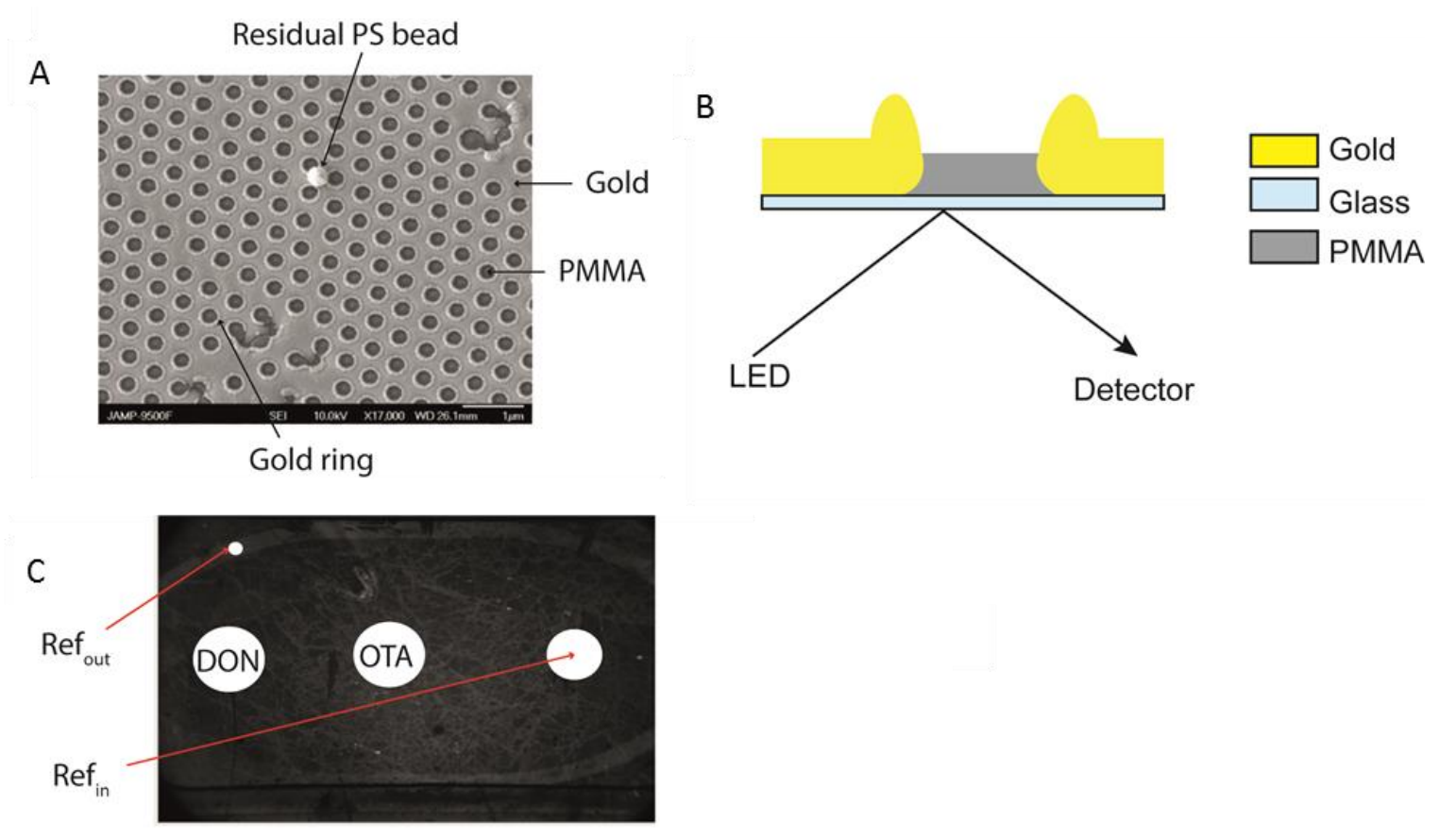

Figure 2. A) Scanning electron microscope image of the nanostructured chip consisting of alternation of gold and PMMA; a residual polystyrene bead can also be seen. B) Schematic representation of the iSPR setup consisting of a nanostructured chip. Note that no prism is used, in contrast to a conventional SPR. and C) iSPR chip as imaged by the instrument displaying the regions of interest (ROI) for DON, OTA, reference outside the flow cell (ref out) and reference inside the flow cell not containing DON or OTA $\left(\right.$ ref $\left._{\text {in }}\right)$. 


\section{Results and discussion}

\section{Biosensor development}

Mycotoxins are small molecules and therefore cannot be detected using a direct SPR assay. In the indirect approach, either the toxin itself or a protein conjugate of the toxin is immobilized on the chip surface. The SPR response of a much larger and specific antibody is then recorded upon injection of a mixture of antibodies and the sample (toxins in buffer or in matrix) over a chip with covalently coupled toxins or toxin-protein conjugates. The assay is based on competition between immobilized mycotoxin and free mycotoxin in solution for binding to the fixed amount of antibody. In our previous study, ${ }^{16}$ ovalbumin conjugates of mycotoxins were immobilized on a $2 \mathrm{D}$ polyethylene glycol surface via the amine group of the protein. In contrast, in the current study direct immobilization of toxins was explored as their binding to the surface is expected to be much more robust compared to protein conjugates, which are more sensitive to the regeneration conditions applied for disruption of antibody binding. In addition, the absence of a big protein, such as ovalbumin, can also contribute to signal enhancement as the SPR sensitivity decreases with increasing distance from the chip surface and is highest close to the surface. However, the immobilization of a small molecule such as a mycotoxin is challenging due to the limited number of functional groups (Figure 1) that can be exploited without blocking the binding sites for antibodies. To efficiently immobilize these small toxins, first a layer of carboxymethylated dextran (CMD) ${ }^{35}$ was bound to the gold surface of the nanostructured iSPR chips. CMD, the most commonly used surface in SPR, is a 3D hydrogel with excellent antifouling properties and has more functional groups compared to the 2D polyethylene glycol layers previously used. ${ }^{16}$ The success of the CMD modification of the nanostructured iSPR chips was confirmed by the low contact angle $\left(<10^{\circ}\right)$ and $X$-ray photoelectron spectroscopy (XPS) results via comparison with conventional flat gold SPR chips modified with the same chemistry by the same manufacturer. The slightly lower degree of modification in case of nanostructured iSPR chips was expected as $20 \%$ of the surface, consisting of PMMA wells in between the gold and PMMA, cannot be modified with CMD. Although there were minor differences in the XPS spectra we were able to successfully immobilize the mycotoxins on the nanostructured chips. DON was chosen as it is the most relevant toxin for beer. ${ }^{5,6}$ On the other hand, OTA, although occurring less frequently in beer, is of concern for the beer industry due to its high toxicity. ${ }^{7,8}$ Both toxins could be successfully immobilized on the CMD surface following our protocol. The multiplexing capability of the iSPR instrument allows the future assay extension with other mycotoxins, depending on the application and prioritized toxin targets. 
The immobilization of the toxins was followed by optimization of several parameters, such as concentration of antibodies, composition of the dilution buffer and regeneration conditions. An optimal concentration of $10 \mu \mathrm{g} / \mathrm{mL}$ for both antibodies in the final sample solution was obtained. The present assay, compared to our previous assay with toxin-ovalbumin conjugates, ${ }^{16}$ requires five times less antibodies to generate a similar iSPR response and is thus much more economical in terms of costly bio-reagent consumption. As reported in other literature ${ }^{36}$ and our previous publication, ${ }^{16}$ a small percentage of methanol is often beneficial for antibody response. Upon testing of antibody binding in HBS-EP buffer containing 0-30\% methanol, 10\% methanol was found to give maximum binding. Similar to our previous assay, $10 \mathrm{mM} \mathrm{HCl} \mathrm{(30} \mathrm{s)} \mathrm{and} 20 \mathrm{mM}$ $\mathrm{NaOH}$ (30 s) was found to be optimal for regeneration of the chips. Calibration curves were recorded for each toxin in antibody solutions in HBS-EP containing $10 \%$ methanol (Figure 3). Additionally, a singleplex calibration curve in buffer was constructed for D3G 4, 3ADON 2 and 15ADON 3 as they are recognized DON conjugates in beer. A favorable cross-reactivity of anti-DON towards the conjugate would allow detection of the sum of both analytes in the SPR assay. Based on the IC50 values of DON ( $200 \mathrm{ng} / \mathrm{mL}$ ), D3G (288 ng/mL), 3ADON (41 ng/mL) and 15ADON (>10000 ng/mL), the obtained crossreactivity for D3G (69\%) is only slightly higher whereas the cross-reactivity for 3ADON $(488 \%)$ is much higher than measured in our earlier assay ( $56 \%$ for D3G and $112 \%$ for 3ADON). ${ }^{16}$ This could be due to the toxins being directly immobilized on the surface instead of via protein conjugates. In turn, this could result in a different part of the toxin being exposed for binding to the antibody. In case of the directly immobilized DON, the 3-acetyl group is clearly beneficial for bio-recognition. As reported by others ${ }^{37,38}$ the cross-reactivity for $15 \mathrm{ADON}$ was found to be negligible $(<2 \%)$.

For measurement of mycotoxins in naturally contaminated samples, usually a matrix matched calibration curve is necessary. Therefore, the relative response, $\left(B / B_{0}\right) \times 100 \%$, in the absence or presence of DON or OTA in buffer and different dilutions of beer was measured (in triplicate) in order to find an appropriate dilution of the beer extract. There was a small difference in response when the dilutions were made in buffer versus in beer. This is expected due to some matrix effect of beer. However, the differences between the $1: 1$ and 1:9 dilutions of beer were insignificant. Therefore, beer diluted $1: 1(\mathrm{v} / \mathrm{v})$ with antibody was arbitrarily selected for the calibration curves. Furthermore, the influence of $\mathrm{pH}$ on the inhibition of the biosensor signal was not significant in the tested $\mathrm{pH}$ range, as was also noted by others in ELISA format. ${ }^{17}$ After these tests, the matrix-matched calibration curves were constructed by simple $1: 1$ dilution of beer with antibody solution without any $\mathrm{pH}$ adjustment. However, adjustment to neutral $\mathrm{pH}$ can be easily performed whenever needed by addition of $1 \mathrm{M}$ sodium carbonate buffer $\mathrm{pH} 9.7$ in case of samples where the beer components especially after 
enrichment give solubility problems. In beer, the calibration curves for both DON and OTA shifted only slightly to the right, thus the matrix effect in case of pale lager beer was minor (Figure 3). Based on the working range of the assay (Table 1), LODs of $17 \mathrm{ng} / \mathrm{mL}$ for DON and $7 \mathrm{ng} / \mathrm{mL}$ for OTA were obtained, thus making the assay suitable for measuring as low as $10 \%$ of TDI depletion for DON (23 ng/mL) whereas for OTA (0.4 $\mathrm{ng} / \mathrm{mL}$ ) a concentration step is required prior to the SPR analysis. This makes the OTA assay somewhat less suitable for field applications. However, after enrichment (75 times), an LOD of $0.09 \mathrm{ng} / \mathrm{mL}$ could be achieved for the OTA assay.

Since SPR assays in beer have never been reported, comparison with literature data is not possible. A recent method for detection of DON in beer using HPLC ${ }^{21}$ was four times less sensitive than our assay while an eight times more sensitive enzyme immunoassay ${ }^{26}$ could be developed only by using a double antibody approach for signal enhancement. Both methods mentioned above, including another one using GC-MS, ${ }^{22}$ involve extensive sample preparation making our assay more suitable for in-field applications. Direct comparison with ELISA is difficult as there are multiple steps involved in ELISA that are not comparable to our assay and could contribute to higher sensitivity. Therefore, comparison with lateral flow immunoassays (LFIs) seems to be most relevant. The reported limit of detection from one of the commercial LFI kits is $100 \mathrm{ng} / \mathrm{mL}$ for DON and $2 \mathrm{ng} / \mathrm{mL}$ for OTA. ${ }^{39}$ Although the LODs were reported for cereals, considering the similarity of our calibration curves in beer and barley, the LODs may be compared. The result is that our biosensor assay for DON is six times more sensitive, while the OTA assay is three times less sensitive.
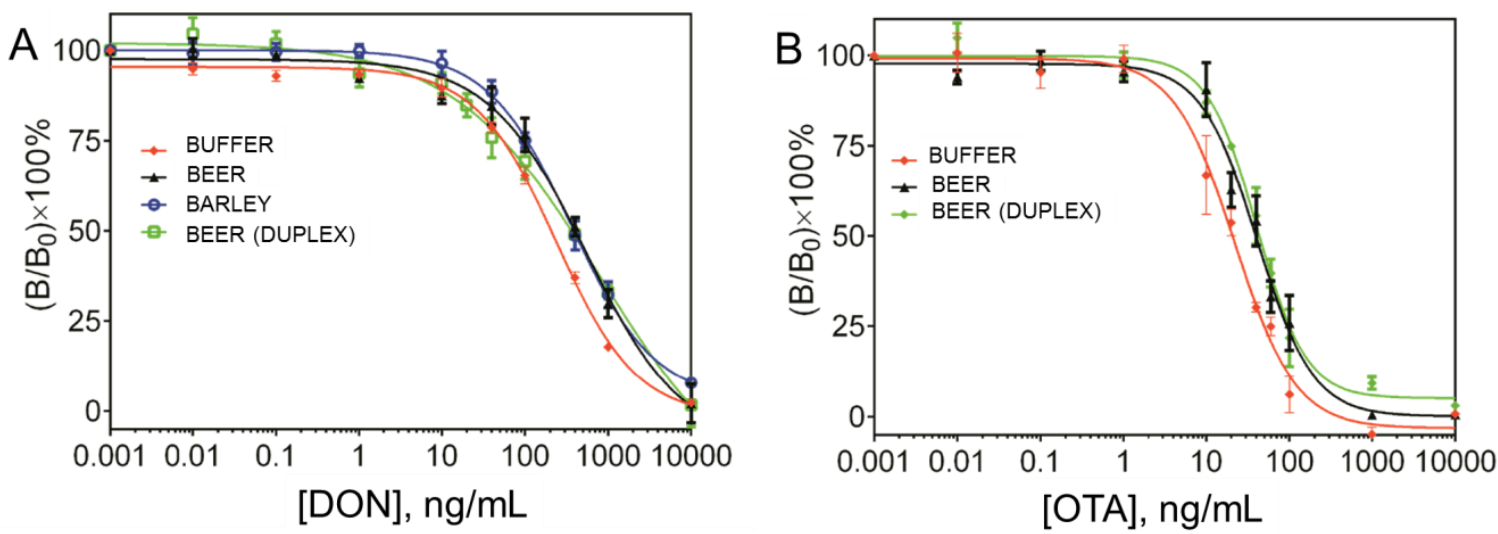

Figure 3. Calibration curves of A) DON and B) OTA in buffer (HBS-EP containing 10\% methanol) and in beer analyzed by iSPR. For DON, a calibration curve in barley extract is also shown $(n=3)$. 


\section{Durability of iSPR chips following multiple analyses and regenerations}

Direct immobilization of mycotoxins instead of their ovalbumin conjugates has greatly improved the durability of the iSPR chips. A single chip was re-used for more than 450 experiments, which is unprecedented for a modified nanostructured sensor chip. After a few initial cycles of regeneration, a perfect regeneration was obtained following each SPR analysis, unlike protein conjugates that were much more sensitive to regenerations. ${ }^{16}$ The durability of the chip helps to reduce the overall cost per assay as the same chip can be used to measure hundreds of samples. After more than 450 cycles, the nanostructured gold started peeling off. The average response of a $10 \mu \mathrm{g} / \mathrm{mL}$ anti-DON over the entire period was $0.027 \pm 0.001(n=32)$ and the final response measured was 0.028 . Therefore, the damage was of a physical nature and related to the chip while the toxins on the surface were still fully functional after 450 cycles.

Table 1. $10 \%$ of tolerable daily intake (TDI) for DON and OTA based on calibration curves in buffer and beer using the iSPR assay $(n=3)$.

\begin{tabular}{|c|c|c|c|c|c|c|c|}
\hline \multirow[b]{2}{*}{ Toxins } & \multirow{2}{*}{$\begin{array}{c}10 \% \text { of TDI } \\
(\mathrm{ng} / \mathrm{mL})\end{array}$} & \multicolumn{3}{|c|}{ Buffer } & \multicolumn{3}{|c|}{ Beer } \\
\hline & & $\operatorname{LOD}^{a}$ & $\begin{array}{r}\text { IC50 } \\
(\mathrm{n}\end{array}$ & $\begin{array}{l}\text { working range } \\
g / m L)\end{array}$ & $\mathrm{LOD}^{\mathrm{a}}$ & $\begin{array}{r}\text { IC50 } \\
(\mathrm{ng}\end{array}$ & $\begin{array}{l}\text { working range } \\
\mathrm{mL} \text { ) }\end{array}$ \\
\hline DON & 23 & 8 & 200 & $36-1000$ & 17 & 400 & $60-2000$ \\
\hline OTA & 0.4 & 3 & 20 & $6-60$ & $\begin{array}{l}7 \\
(0.09 *)\end{array}$ & 40 & $10-120$ \\
\hline
\end{tabular}

${ }^{a}$ LOD is defined as IC10 (concentration at which $10 \%$ inhibition of binding occurs)

${ }^{b}$ Working range is defined as IC20-IC80 (concentration at which $20 \%-80 \%$ inhibition of binding occurs)

*LOD after 75 times enrichment

\section{Preliminary in-house validation}

To check the repeatability of inhibition at the considered theoretical safe limits (TSLs, $120 \mathrm{ng} / \mathrm{mL}$ for DON), blank beer from one bottle of pale lager was spiked and both the blank and spiked beer were measured in five repeats on the same day. The same experiment (including spiking) was repeated for another two days to get 15 repeats. The average relative responses (\%) of blank and spiked samples were $101.9 \pm 4.6$ and 77.1 \pm 2.8 , respectively, thus highlighting the excellent repeatability of the inhibition assay. Furthermore, based on the obtained data, the intra- and inter-day RSDs were calculated. Since the samples were divided over three days, the intra-day RSDs are an average of the RSDs of three days while the inter-day RSDs were the deviations of the average responses of the three days. The obtained intra-day and inter-day variation for the response of blank beer were $4.4 \%$ and $5.6 \%$ respectively while those of spiked beer were 
2.8 and $3.9 \%$ respectively. Preliminary in-house validation was then performed at the considered TSLs (120 ng/mL for DON and $0.2 \mathrm{ng} / \mathrm{mL}$ for OTA) using 20 different blank and spiked beers. In case of DON, simple dilution 90:1:9) (beer:anti-DON:methanol) was enough to allow detection within the working range of the assay (Figure 4A). However, for the detection of OTA at the desired TSL $(0.2 \mathrm{ng} / \mathrm{mL})$ and considering the working range of our calibration curve in beer (10-120 ng/mL), sample pre-concentration was required. For a successful validation, the cut-off factor $(\mathrm{Fm})$ values have to be lower than the threshold $(\mathrm{T})$ values and only $5 \%$ of the spiked samples ( 1 out of 20 ) are allowed to be false negative. Having fulfilled both these criteria, our method was successfully validated at the considered TSLs for both DON and OTA (Figure 4). Any sample with a relative response below the cut-off value would be considered 'screen positive' and should be subjected to further confirmatory analysis. The obtained RSDs for different batches of blank and beers spiked with DON were compared to the earlier experiment where beer from one bottle of pale lager beer was used. Despite the different batch numbers as an additional variable, the intra- and inter-day RSDs were very similar to the earlier experiment for blank beer (4.6 and 5.5\% respectively) while the values for spiked beer were only slightly higher (4.7 and 4.3\% respectively). Similar RSDs (6.5-5.1\%) were obtained for blank and beer spiked with OTA in the latter experiment. The obtained results show that the developed assay is reproducible, robust and suitable for detection of DON and OTA in beer.

\section{Measurement of naturally contaminated beer samples}

A few samples of beer naturally contaminated with DON and D3G were analyzed to demonstrate the real-life application of the developed assay. Since, the anti-DON has high cross-reactivity towards D3G, concentrations of both toxins have to be considered during analysis. Based on the values obtained by LC-MS/MS, beer 1 (diluted 5 times in buffer) was mixed $1: 1$, beer 2 was mixed 9:1 and beer 3 was mixed $1: 1$ with anti-DON to obtain the iSPR response. For contaminated beer 1 (CB1) containing $664 \mathrm{ng} / \mathrm{mL}$ $\mathrm{DON}+\mathrm{D} 3 \mathrm{G},{ }^{19}$ the measured inhibition for the contaminated sample corresponded to a concentration of $529 \pm 37 \mathrm{ng} / \mathrm{mL}$, i.e., $20 \%$ lower than the LC-MS/MS value. In case of CB2 and CB3, the measured concentrations of $169 \pm 34$ and $292 \pm 65 \mathrm{ng} / \mathrm{mL}$ were 20 and $30 \%$ higher, respectively, than the LC-MS/MS values $(140 \mathrm{ng} / \mathrm{mL}$ and $225 \mathrm{ng} / \mathrm{mL}$ ). The higher concentrations measured in the latter two cases could be due to presence of other conjugates of DON having cross-reactivity towards anti-DON, at quantities below the detection limit of the LC-MS/MS method. Such results have also been observed in an earlier study where the concentrations obtained from ELISA were higher than those obtained from LC-MS/MS. ${ }^{17}$ However, such overestimation would not affect a screening method like the one presented here where a sample with relative responses below the 
cut-off value would be considered as 'screen positive' and would be subjected to LCMS/MS analysis anyway.

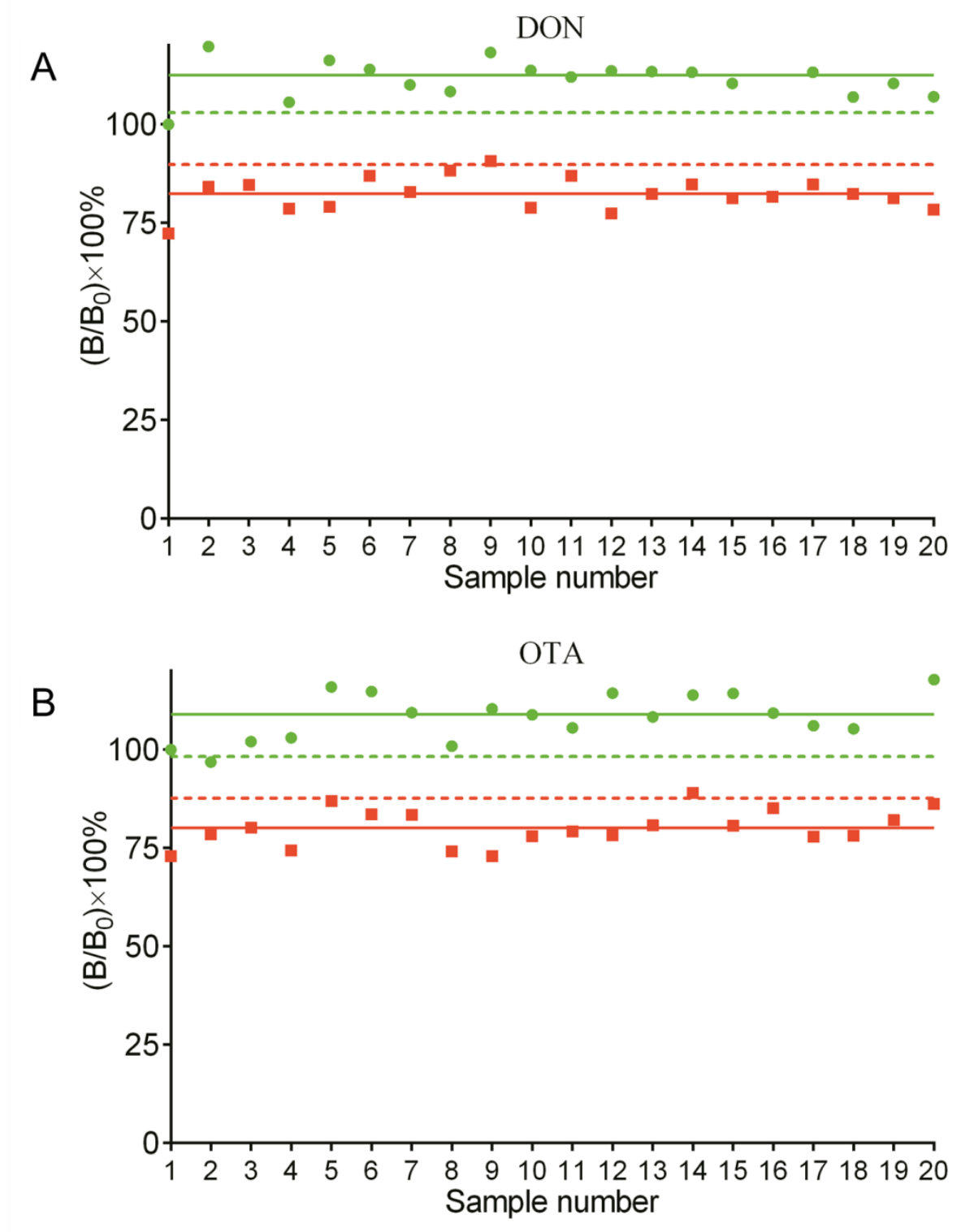

Figure 4. Validation graphs for A) DON and B) OTA in 20 different batches of pale lager beer analyzed by iSPR. The responses of the blank samples (green dots) and spiked samples (red squares) are relative to the response of the blank beer 1 . The averages of the responses of the blank samples (green solid line) and spiked samples (red solid line), the threshold values (green dotted line) and cut-off factors (red dotted line) are also shown in the graph.

\section{Feasibility of multiplexing}

To assess the feasibility of the assay for multiplex detection of DON and OTA, multiplex calibration curves with both antibodies and toxins were constructed. The minor differences with the singleplex calibration curves (Figure 3 ) prove that the assay can be easily transferred to a multiplex format. Keeping the multiplexing option in mind, a beer sample spiked with both DON and OTA at their considered TSLs was subjected to the SPE 
protocol as described for OTA. Firstly, iSPR analysis of the flow-through, collected during loading of beer, was done to confirm that DON was not retained by the SPE column and was present in the flow-through collected during loading. Next, the beer extract (containing OTA), after the entire SPE procedure and evaporation, was re-dissolved in $900 \mu \mathrm{L}$ of the flow-through (containing DON). Finally, $10 \mu \mathrm{L}$ of anti-DON and anti-OTA $(100 \mu \mathrm{g} / \mathrm{mL}$ in HBS-EP of each) plus $90 \mu \mathrm{L}$ of methanol was added to measure the concentration of both toxins in a single experiment. The final concentrations for the iSPR measurements were $10 \mu \mathrm{g} / \mathrm{mL}$ of anti-DON and $10 \mu \mathrm{g} / \mathrm{mL}$ of anti-OTA plus $108 \mathrm{ng} / \mathrm{mL}$ of DON and $15 \mathrm{ng} / \mathrm{mL}$ of OTA in HBS-EP containing $9 \%$ methanol. The measured relative responses (75 $\pm 4 \%$ for DON and $74 \pm 8 \%$ for OTA) were identical to the expected responses ( $77 \pm 3 \%$ for DON and $78 \pm 5 \%$ for OTA) obtained from multiplex calibration curves, showing that simultaneous measurement of the two toxins is possible.

\section{Measurement of other beers}

To demonstrate the versatility of the assay in beer analysis, ten different beers (with different styles and/or alcohol percentages) from the Dutch market were analyzed in triplicate. All the beers were spiked with DON and OTA at the considered TSLs and the average relative responses for all beer types were below the cut-off value (Figure 5). Thus, the developed assay is applicable to beer within a range of styles (pale lager, pilsner, Czech pilsner, abbey dubbel and abbey triple) and alcohol percentages (0-9.5\%), despite the fact that the matrix matched calibration curve was only in pale lager beer containing $5 \%$ alcohol.

\section{At-line testing of beers at a commercial brewery}

To demonstrate the applicability for DON testing, the iSPR instrument was transported to a brewery. Within 15 min the instrument was ready to measure and a number of different commercial beers were measured for the presence of DON. The only sample pretreatment required was degassing. After adding anti-DON, beers could be measured every 15 min including regeneration. Per sample, $100 \mu \mathrm{L}$ of beer was needed for one measurement. All of the beers, using a calibration curve measured one week earlier in our lab, were negative for DON (<LOD of $17 \mathrm{ng} / \mathrm{mL}$ ). However after spiking with DON at the TSL $(120 \mathrm{ng} / \mathrm{mL})$, all beers were positive. The \% inhibition of the iSPR signal for the beer spiked at the brewery deviated less than $10 \%$ from values recorded with other beers spiked at the same level in our own laboratory. This clearly shows the transferability of the methodology. 


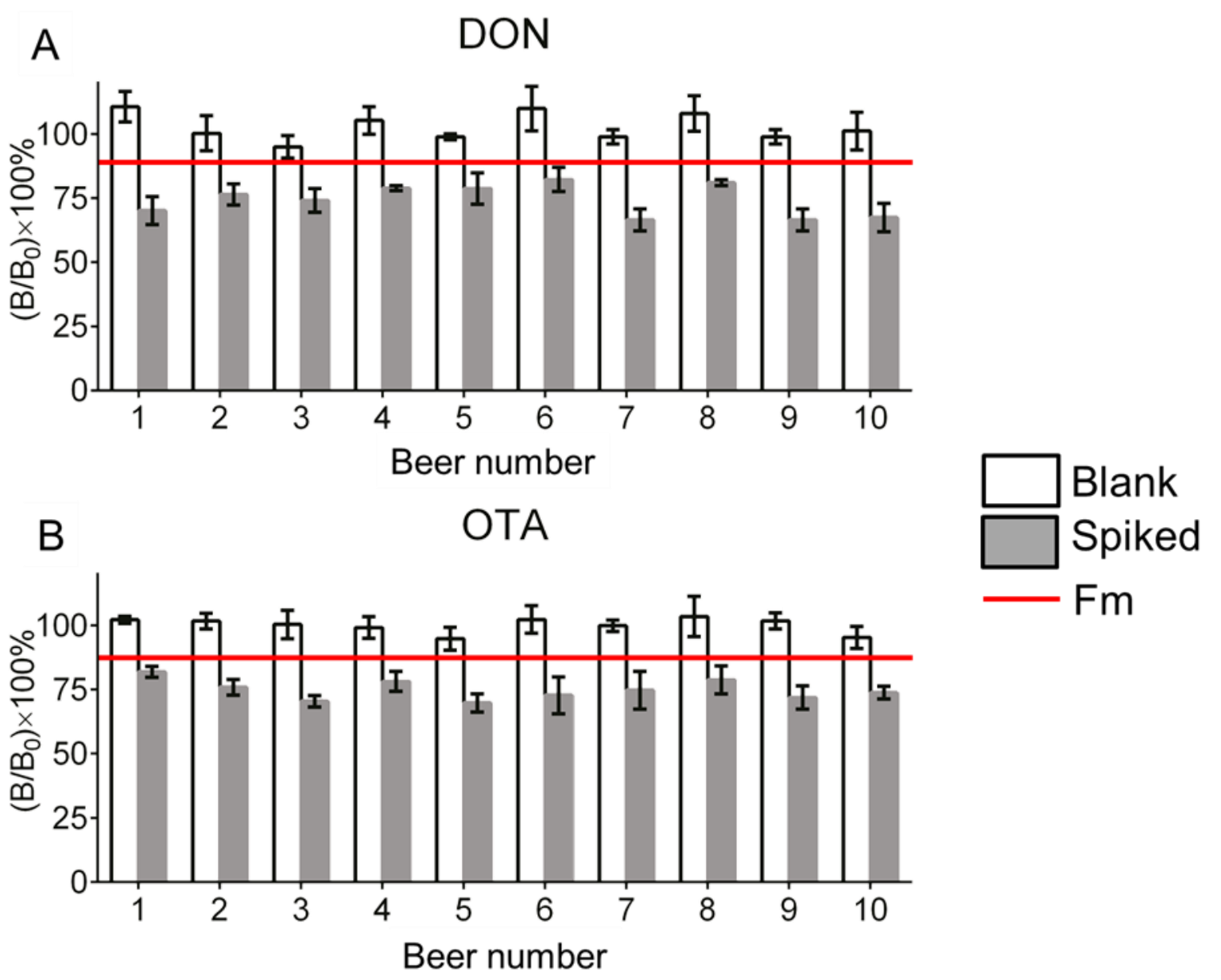

Figure 5. Relative response for ten different beers with and without A) DON and B) OTA measured using the iSPR instrument. The red line is the cut-off factor (Fm) obtained from validation. For each beer type, the responses are an average of triplicate measurement of the same sample and each response is relative to the response of the first out of three replicates for blank beer of that type.

\section{Measurement of barley}

The newly developed biosensor immunoassay requires no sample preparation other than degassing for the analysis of DON in beer. Therefore, it was interesting to see whether the assay is applicable to beer ingredients as well. To test the possible use of the assay for in-field detection of DON in barley, we optimized and simplified our previous extraction protocol $^{16}$ for barley. A calibration curve was constructed using the barley extract (Figure $3 \mathrm{~A}$ ), and this turned out to be highly similar to the one in beer. The new protocol can be considered a major improvement over our previous method, in which calibration was time consuming due to the long extraction procedure. Based on the calibration curve, a limit of detection of $30 \mathrm{ng} / \mathrm{mL}$, an IC50 of $400 \mathrm{ng} / \mathrm{mL}$ and a working range of $76-2000 \mathrm{ng} / \mathrm{mL}$ were obtained. Finally, the recovery of the extraction protocol was tested using barley samples spiked at the EU maximum level (ML) of $1250 \mu \mathrm{g} / \mathrm{kg}$. The relative response recorded $(74 \% \pm 3)$ corresponds to a concentration of $874 \pm 22$ 
$\mu \mathrm{g} / \mathrm{kg}$, i.e., $30 \%$ lower than the expected value at $100 \%$ recovery. This could be due to incomplete recovery, as was also seen in our previous study. ${ }^{16}$ Nevertheless, a significant inhibition was seen for barley spiked at ML, which makes this assay suitable for semiquantitative screening of DON in barley. The simplified extraction protocol involves a short extraction step followed by filtration and does not require any sophisticated laboratory equipment and can be easily performed in the field.

In summary, a competitive inhibition immunoassay using a portable iSPR instrument was successfully developed for the detection of DON and OTA in beer. In contrast to our previous assay using PEG3500 with OVA conjugates of mycotoxins, ${ }^{16} 3 \mathrm{D}$ carboxymethylated dextran was used for direct attachment of toxins in the present assay. This has helped to reduce the concentration of antibodies by a factor of five and to increase the durability of the nanostructured biosensor chip by 7.5 times. The assay LOD allows detection of even less than $10 \%$ TDI depletion of both DON and OTA. A preliminary in-house validation based on 20 different batches of beer, along with measurement of ten additional beer samples of different styles and alcohol percentages, and of naturally contaminated beer samples, demonstrates the wide applicability and robustness of the assay for the detection of the two most relevant toxins in beer. The observed cross-reactivity of anti-DON towards D3G and 3ADON allows determination of the sum of the analytes. Furthermore, the DON assay requires minimal sample preparation compared to methods reported in the literature $21,22,26$ and can also be easily extended to the detection in barley and in compliance with the legal limit set by the EU. The multiplexing capability of the iSPR would allow extension of the current assay to other mycotoxins while the portability of the instrument and the transferability of the method were proven by a successful at-line application at a commercial brewery.

\section{Acknowledgements}

This research received funding from the Netherlands Organisation for Scientific Research (NWO) in the framework of the Technology Area COAST (project nr 053.21.107) with WU, VU Amsterdam, RIKILT, Heineken, Synthon, Technex, EuroProxima, Waterproef as partners and Plasmore and Bionavis as associated partners. Willem Haasnoot (RIKILT, Wageningen, NL) is gratefully acknowledged for his suggestion of direct immobilization of the toxins. We would like to thank Milena Zachariasova (UCT Prague, Czech Republic) and Jeroen Peters (RIKILT) for the contaminated beer samples and project partners for stimulating discussions. 


\section{References}

1. DeVries, J. W.; Trucksess, M. W.; Jackson, L. S., Mycotoxins and food safety. Springer: New York, 2002.

2. Lancova, K.; Hajslova, J.; Poustka, J.; Krplova, A.; Zachariasova, M.; Dostalek, P.; Sachambula, L. Transfer of Fusarium mycotoxins and 'masked' deoxynivalenol (deoxynivalenol-3-glucoside) from field barley through malt to beer. Food Addit. Contam., Part A 2008, 25, 732-744.

3. Inoue, T.; Nagatomi, Y.; Uyama, A.; Mochizuki, N. Fate of mycotoxins during beer brewing and fermentation. Biosci., Biotechnol., Biochem. 2013, 77, 1410-1415.

4. Habler, K.; Hofer, K.; Geissinger, C.; Schuler, J.; Huckelhoven, R.; Hess, M.; Gastl, M.; Rychlik, M. Fate of Fusarium toxins during the malting process. J. Agric. Food Chem. 2016, $64,1377-1384$.

5. Curtui, V.; Brockmeyer, A.; Dietrich, R.; Kappenstein, O.; Klaffke, H.; Lepschy, J.; Märtlbauer, E.; Schneider, E.; Seidler, C.; Thielert, G.; Usleber, E.; Weber, R.; Wolff, J. Deoxynivalenol in food. Mycotoxin Res. 2005, 21, 83-88.

6. Kostelanska, M.; Hajslova, J.; Zachariasova, M.; Malachova, A.; Kalachova, K.; Poustka, J.; Fiala, J.; Scott, P. M.; Berthiller, F.; Krska, R. Occurrence of deoxynivalenol and its major conjugate, deoxynivalenol-3-glucoside, in beer and some brewing intermediates. J. Agric. Food Chem. 2009, 57, 3187-3194.

7. Mateo, R.; Medina, Á.; Mateo, E. M.; Mateo, F.; Jiménez, M. An overview of ochratoxin A in beer and wine. Int. J. Food Microbiol. 2007, 119, 79-83.

8. Bellver Soto, J.; Fernández-Franzón, M.; Ruiz, M.-J.; Juan-García, A. Presence of ochratoxin A (OTA) mycotoxin in alcoholic drinks from southern European countries: Wine and beer. $J$. Agric. Food Chem. 2014, 62, 7643-7651.

9. Commission regulation (EC) No 1881/2006 of 19 December 2006 setting maximum levels for certain contaminants in foodstuffs. Off. J. Eur. Union 2006, L364, 5-24.

10. Scientific committee on food opnion on Fusarium toxins. Part 1: Deoxynivalenol (DON) (expressed on 2 December 1999). http://ec.europa.eu/food/fs/sc/scf/out44_en.pdf (Accessed: 10 September 2016).

11. European Food Safety. Opinion of the scientific panel on contaminants in the food chain on a request from the commission related to ochratoxin $A$ in food. EFSA J. 2006, 4, 1-56.

12. Berthiller, F.; Brera, C.; Crews, C.; Iha, M. H.; Krska, R.; Lattanzio, V. M. T.; MacDonald, S.; Malone, R. J.; Maragos, C.; Solfrizzo, M.; Stroka, J.; Whitaker, T. B. Developments in mycotoxin analysis: An update for 2013-2014. World Mycotoxin J. 2015, 8, 5-35.

13. Berthiller, F.; Brera, C.; Crews, C.; Iha, M. H.; Krska, R.; Lattanzio, V. M. T.; MacDonald, S.; Malone, R. J.; Maragos, C.; Solfrizzo, M.; Stroka, J.; Whitaker, T. B. Developments in mycotoxin analysis: An update for 2014-2015. World Mycotoxin J. 2016, 9, 5-30.

14. Chauhan, R.; Singh, J.; Sachdev, T.; Basu, T.; Malhotra, B. D. Recent advances in mycotoxins detection. Biosens. Bioelectron. 2016, 81, 532-545.

15. Dai, S.; Wu, S.; Duan, N.; Wang, Z. A luminescence resonance energy transfer based aptasensor for the mycotoxin ochratoxin A using upconversion nanoparticles and gold nanorods. Microchim. Acta 2016, 183, 1909-1916.

16. Joshi, S.; Segarra-Fas, A.; Peters, J.; Zuilhof, H.; van Beek, T. A.; Nielen, M. W. F. Multiplex surface plasmon resonance biosensing and its transferability towards imaging nanoplasmonics for detection of mycotoxins in barley. Analyst 2016, 141, 1307-1318.

17. Zachariasova, M.; Hajslova, J.; Kostelanska, M.; Poustka, J.; Krplova, A.; Cuhra, P.; Hochel, I. Deoxynivalenol and its conjugates in beer: A critical assessment of data obtained by enzyme-linked immunosorbent assay and liquid chromatography coupled to tandem mass spectrometry. Anal. Chim. Acta 2008, 625, 77-86.

18. Zachariasova, M.; Cajka, T.; Godula, M.; Malachova, A.; Veprikova, Z.; Hajslova, J. Analysis of multiple mycotoxins in beer employing (ultra)-high-resolution mass spectrometry. Rapid Commun. Mass Spectrom. 2010, 24, 3357-3367.

19. Zachariasova, M.; Vaclavikova, M.; Lacina, O.; Vaclavik, L.; Hajslova, J. Deoxynivalenol oligoglycosides: new "masked" Fusarium toxins occurring in malt, beer, and breadstuff. J. Agric. Food Chem. 2012, 60, 9280-9291.

20. Varga, E.; Malachova, A.; Schwartz, H.; Krska, R.; Berthiller, F. Survey of deoxynivalenol and its conjugates deoxynivalenol-3-glucoside and 3-acetyl-deoxynivalenol in 374 beer samples. Food Addit. Contam., Part A 2013, 30, 137-146.

21. Piacentini, K. C.; Savi, G. D.; Olivo, G.; Scussel, V. M. Quality and occurrence of deoxynivalenol and fumonisins in craft beer. Food Control 2015, 50, 925-929. 
22. Rodríguez-Carrasco, Y.; Fattore, M.; Albrizio, S.; Berrada, H.; Mañes, J. Occurrence of Fusarium mycotoxins and their dietary intake through beer consumption by the European population. Food Chem. 2015, 178, 149-155.

23. Li, W.; Powers, S.; Dai, S. Y. Using commercial immunoassay kits for mycotoxins: 'joys and sorrows'? World Mycotoxin J. 2014, 7, 417-430.

24. Papadopoulou-Bouraoui, A.; Vrabcheva, T.; Valzacchi, S.; Stroka, J.; Anklam, E. Screening survey of deoxynivalenol in beer from the European market by an enzyme-linked immunosorbent assay. Food Addit. Contam., Part A 2004, 21, 607-617.

25. Kuzdraliński, A.; Solarska, E.; Muszyńska, M. Deoxynivalenol and zearalenone occurence in beers analysed by an enzyme-linked immunosorbent assay method. Food Control 2013, 29, 22-24.

26. Bauer, J. I.; Gross, M.; Gottschalk, C.; Usleber, E. Investigations on the occurrence of mycotoxins in beer. Food Control 2016, 63, 135-139.

27. Meneely, J. P.; Elliott, C. T. Rapid surface plasmon resonance immunoassays for the determination of mycotoxins in cereals and cereal-based food products. World Mycotoxin J. 2014, 7, 491-505.

28. Giudicatti, S.; Valsesia, A.; Marabelli, F.; Colpo, P.; Rossi, F. Plasmonic resonances in nanostructured gold/polymer surfaces by colloidal lithography. Phys. Status Solidi A 2010, 207, 935-942.

29. Joshi, S.; Pellacani, P.; van Beek, T. A.; Zuilhof, H.; Nielen, M. W. F. Surface characterization and antifouling properties of nanostructured gold chips for imaging surface plasmon resonance biosensing. Sens. Actuators, B 2015, 209, 505-514.

30. Aqai, P.; Peters, J.; Gerssen, A.; Haasnoot, W.; Nielen, M. W. F. Immunomagnetic microbeads for screening with flow cytometry and identification with nano-liquid chromatography mass spectrometry of ochratoxins in wheat and cereal. Anal. Bioanal. Chem. 2011, 400, 3085-3096.

31. Meneely, J. P.; Quinn, J. G.; Flood, E. M.; Hajšlová, J.; Elliott, C. T. Simultaneous screening for T-2/HT-2 and deoxynivalenol in cereals using a surface plasmon resonance immunoassay. World Mycotoxin J. 2012, 5, 117-126.

32. Valsesia, A.; Marabelli, F.; Giudicatti, S.; Marchesini, G. R.; Rossi, F.; Colpo, P. SPR sensor device with nanostructure. World Patent 2013, WO 2013/007448 A1.

33. Bottazzi, B.; Fornasari, L.; Frangolho, A.; Giudicatti, S.; Mantovani, A.; Marabelli, F.; Marchesini, G.; Pellacani, P.; Therisod, R.; Valsesia, A. Multiplexed label-free optical biosensor for medical diagnostics. J. Biomed. Opt. 2014, 19, 017006-017010.

34. Commission regulation (EU) no $519 / 2014$ of 16 May 2014 amending regulation (EC) no $401 / 2006$ as regards methods of sampling of large lots, spices and food supplements, performance criteria for T-2, HT-2 toxin and citrinin and screening methods of analysis. Off. J. Eur. Union 2014, L147, 29-43.

35. Löfås, S.; Johnsson, B. A novel hydrogel matrix on gold surfaces in surface plasmon resonance sensors for fast and efficient covalent immobilization of ligands. J. Chem. Soc., Chem. Commun. 1990, 1526-1528.

36. Skládal, P. Effect of methanol on the interaction of monoclonal antibody with free and immobilized atrazine studied using the resonant mirror-based biosensor. Biosens. Bioelectron. 1999, 14, 257-263.

37. Kadota, T.; Takezawa, Y.; Hirano, S.; Tajima, O.; Maragos, C. M.; Nakajima, T.; Tanaka, T.; Kamata, Y.; Sugita-Konishi, Y. Rapid detection of nivalenol and deoxynivalenol in wheat using surface plasmon resonance immunoassay. Anal. Chim. Acta 2010, 673, 173-178.

38. Peters, J.; Cardall, A.; Haasnoot, W.; Nielen, M. W. F. 6-Plex microsphere immunoassay with imaging planar array detection for mycotoxins in barley. Analyst 2014, 139, 3968-3976.

39. Charms Science, Inc., ROSA Mycotoxin https://www.charm.com/products/mycotoxins/rosa-mycotoxin-strips (Accessed: 02 May 2016). 


\section{Supporting information}

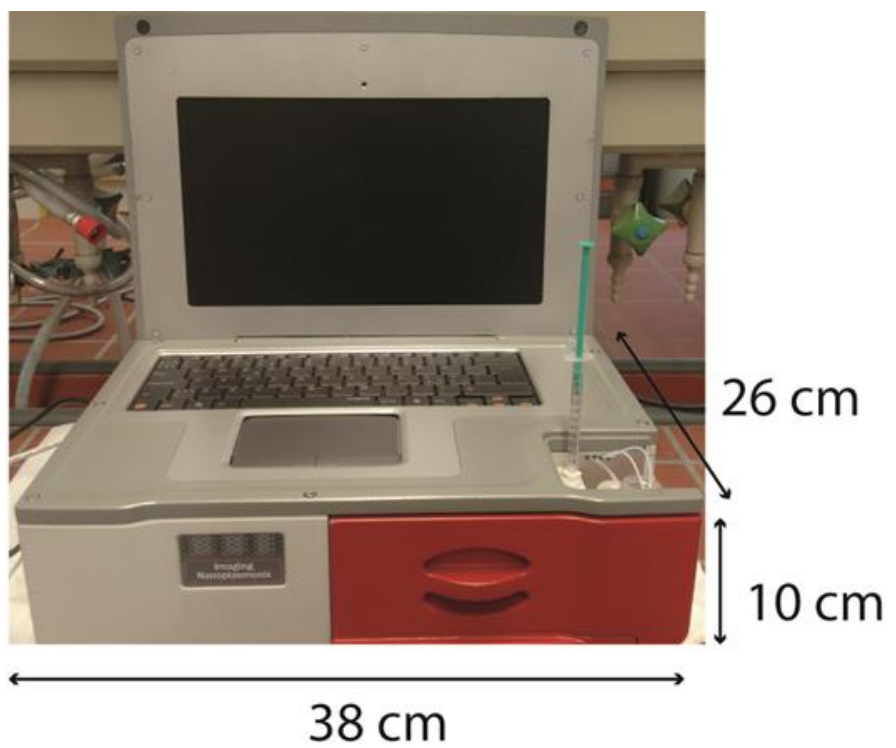

Figure S1. Picture of the nanostructured iSPR instrument used in this study.

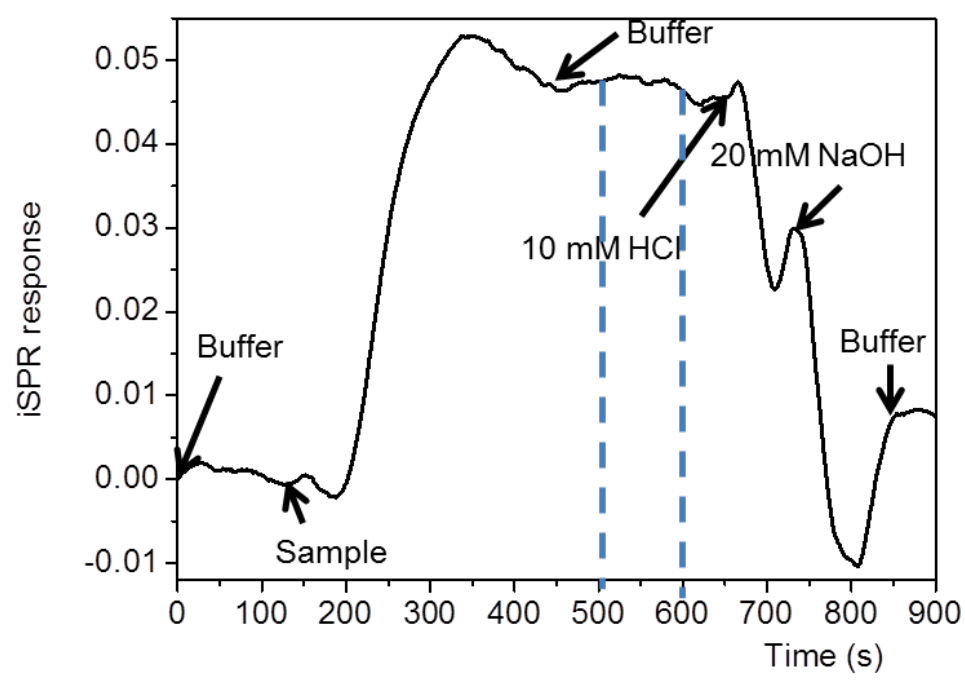

Figure S2. Typical sensorgram generated upon injection of $10 \mu \mathrm{g} / \mathrm{ml}$ anti-OTA measured using a prototype iSPR instrument with covalently coupled OTA to a CMD surface on a nanostructured biosensor chip. Each cycle consists of flushing with buffer, injection of sample (antibody mixed with buffer or beer), buffer and regeneration with $10 \mathrm{mM} \mathrm{HCl}(30 \mathrm{~s})$ followed by $20 \mathrm{mM} \mathrm{NaOH}(30 \mathrm{~s})$. After injection of sample and regeneration, the chip was flushed with buffer before starting a new cycle. The two dashed blue lines show the range where the average iSPR response was taken. 

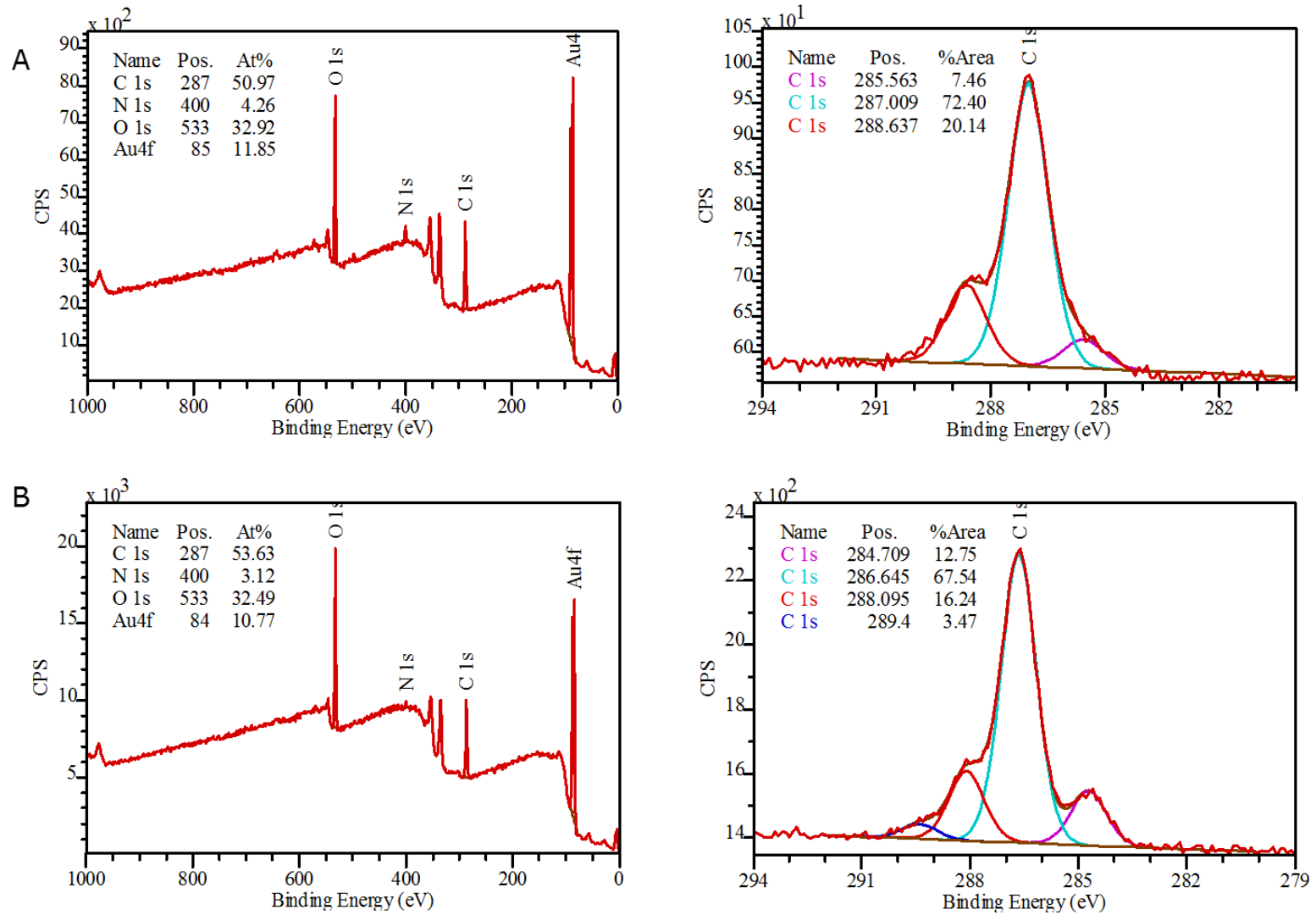

Figure S3. XPS survey (left) and C1s narrow scan (right) spectra of A) nanostructured iSPR chip and B) flat SPR chip, both modified with carboxymethylated dextran.

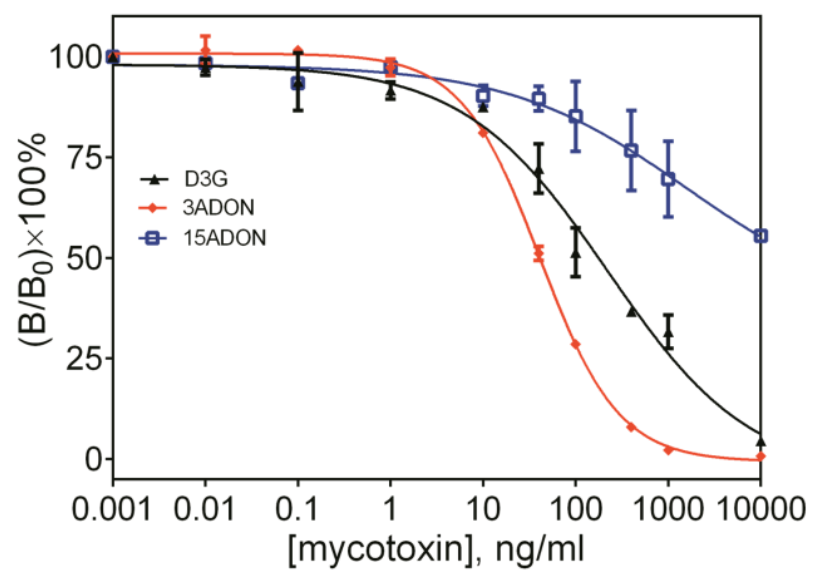

Figure S4. Calibration curves of conjugates of DON: D3G, 3ADON and 15ADON in buffer (HBS-EP containing $10 \%$ methanol) analyzed by iSPR. $20 \mu \mathrm{g} / \mathrm{mL}$ of anti-DON was mixed $1: 1$ with toxin conjugates to obtain the responses for the calibration curves $(n=3)$. 


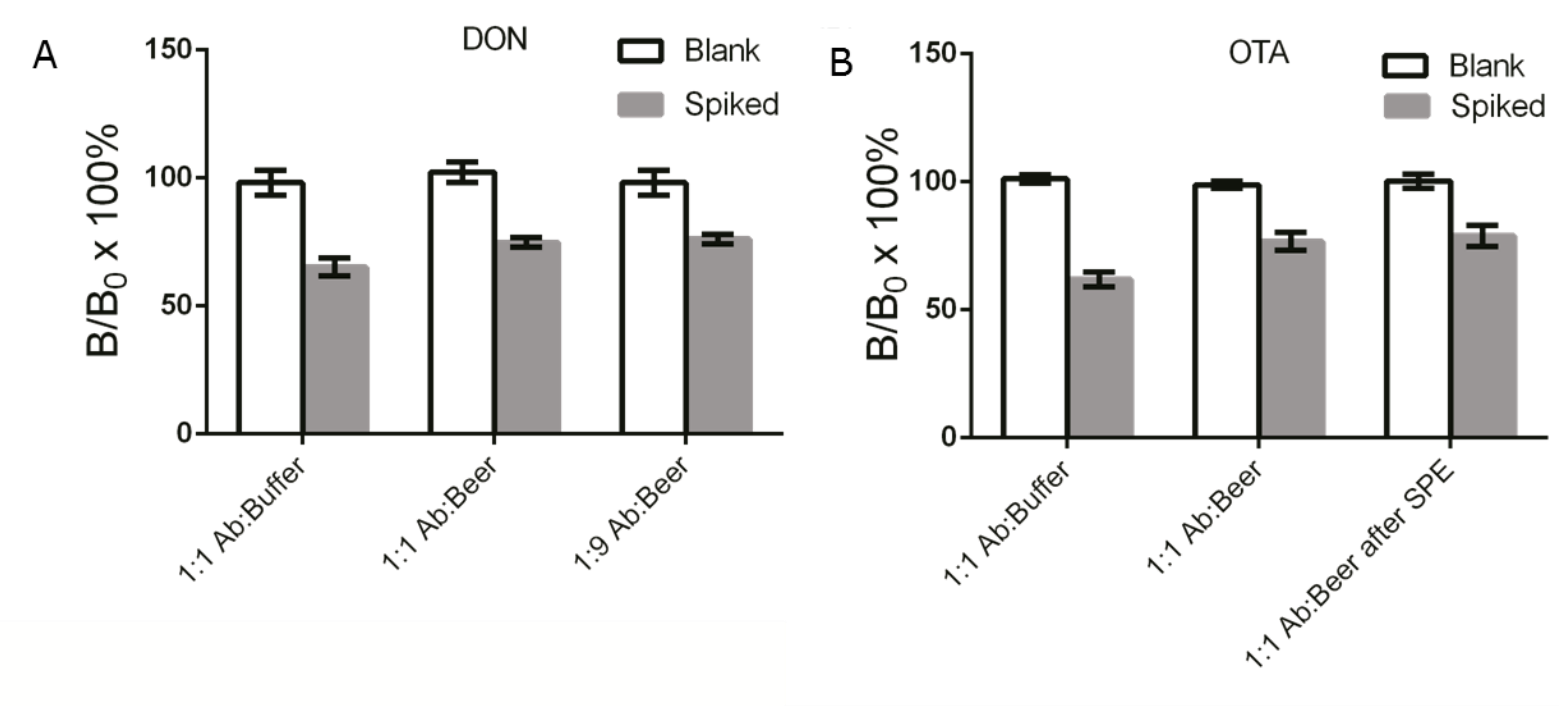

Figure S5. Relative iSPR responses measured for $A$ ) anti-DON (Ab) solution upon mixing 1:1 with buffer (HBS-EP containing 10\% methanol), $1: 1$ with beer and 1:9 with beer and B) anti-OTA (Ab) upon mixing 1:1 with buffer (HBS-EP containing 10\% methanol), beer, and beer after SPE. In all experiments, the final concentration of $A b$ was $10 \mu \mathrm{g} / \mathrm{mL}$. The spiked sample had a final concentration of $108 \mathrm{ng} / \mathrm{mL}$ of DON or $15 \mathrm{ng} / \mathrm{mL}$ of OTA. Each bar is an average of triplicate measurements of a sample with the standard deviation shown as error bars. Each response is relative to the response of first replicate out of the three for blank beer of each dilution.
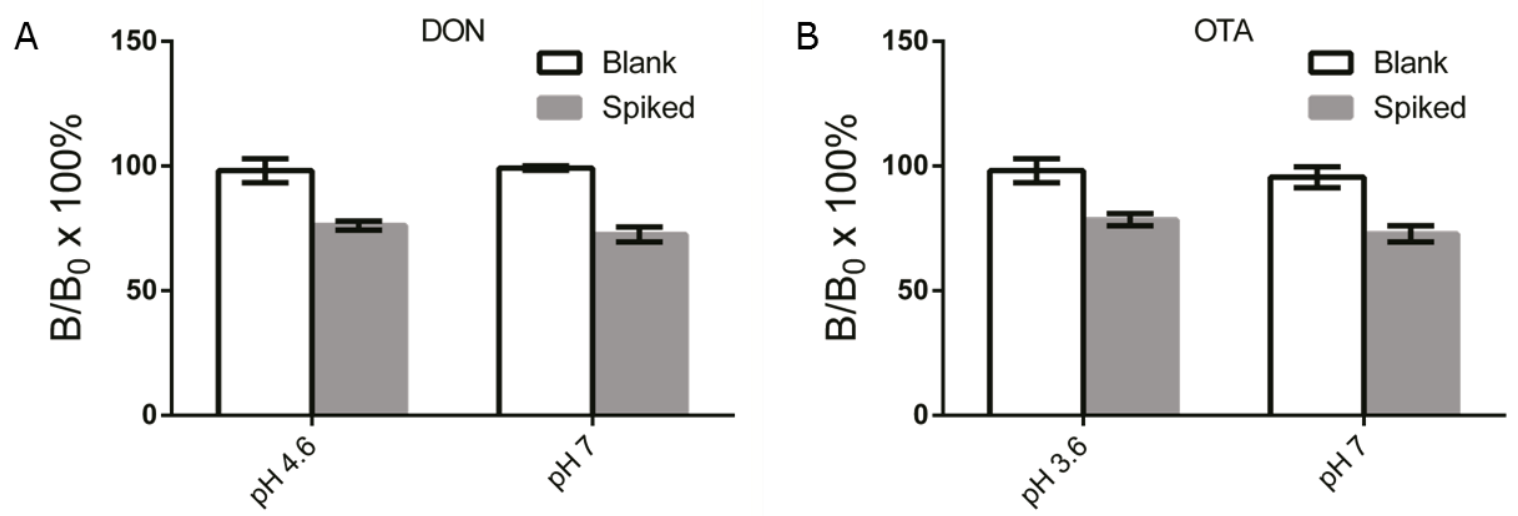

Figure S6. Relative iSPR responses measured for A) $100 \mu \mathrm{g} / \mathrm{mL}$ anti-DON upon mixing $1: 9(\mathrm{v} / \mathrm{v})$ with blank and spiked beer at pH 4.6 and pH 7 B) $20 \mu \mathrm{g} / \mathrm{mL}$ anti-OTA upon mixing $1: 1(\mathrm{v} / \mathrm{v})$ with blank and spiked beer obtained after SPE at $\mathrm{pH} 3.6$ and $\mathrm{pH}$ 7. In all experiments, the final concentration of antibody was $10 \mu \mathrm{g} / \mathrm{mL}$. The spiked sample had a final concentration of 108 $\mathrm{ng} / \mathrm{mL}$ of DON or $15 \mathrm{ng} / \mathrm{mL}$ of OTA. The first $\mathrm{pH}$ (4.6 for DON and 3.6 for OTA) values were obtained after mixing the corresponding solution with antibody solution while $\mathrm{pH} 7$ was achieved by adjusting the $\mathrm{pH}$ of the mixed solution using $1 \mathrm{M}$ sodium carbonate buffer $\mathrm{pH}$ 9.7. Each bar is an average of triplicate measurements of a sample with the standard deviation shown as error bars. Each response is relative to the response of first replicate out of the three for blank beer of each pH category. 
Table S1. Intra- and inter-Day precision of the mycotoxin assay performed on four different days for blank and spiked beer samples

\begin{tabular}{llll}
\hline \multirow{2}{*}{ sample } & Precision & \multicolumn{2}{l}{ RSD for each assay in \% } \\
\cline { 3 - 4 } & DON & OTA \\
\hline \multirow{2}{*}{ blank } & intra-day $(4 \times n=5)$ & 4.6 & 5.4 \\
& inter-day $(n=4)$ & 5.5 & 6.5 \\
\hline \multirow{2}{*}{ spiked } & intra-day $(4 \times n=5)$ & 4.7 & 6.2 \\
& inter-day $(n=4)$ & 4.3 & 5.1 \\
\hline
\end{tabular}




\title{
Chapter 5
}

\section{Biochip spray: Simplified coupling of surface plasmon resonance biosensing and mass spectrometry}

\author{
Sweccha Joshi ${ }^{1,2}$, Han Zuilhof ${ }^{1}$, Teris A. van Beek ${ }^{1}$, Michel W.F. Nielen ${ }^{1,3}$ \\ ${ }^{1}$ Laboratory of Organic Chemistry, Wageningen University \& Research, Stippeneng 4, \\ 6708 WE Wageningen, The Netherlands \\ ${ }^{2}$ TI-COAST, Science Park 904, 1098 XH Amsterdam, The Netherlands \\ ${ }^{3}$ RIKILT Wageningen University \& Research, P.O. Box 230, 6700 AE Wageningen, The \\ Netherlands
}

This chapter has been published in:

Analytical Chemistry, 2017, doi: 10.1021/acs.analchem.6b04012.
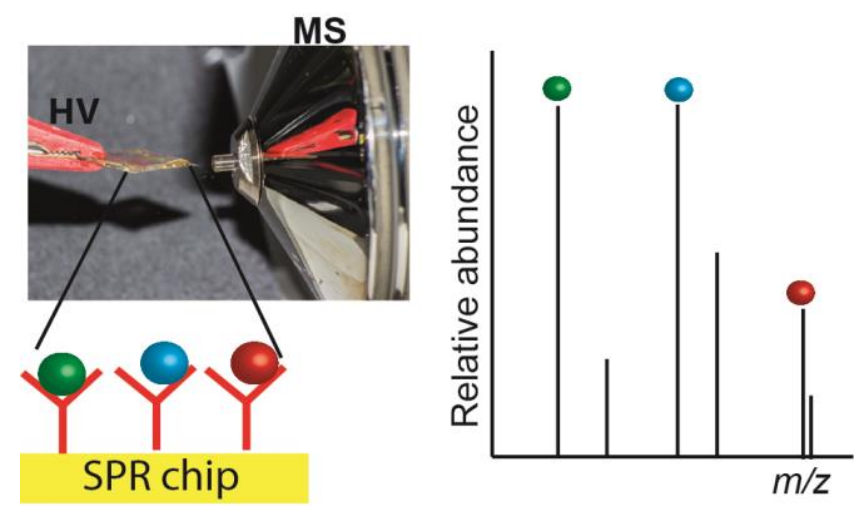


\section{Abstract}

A simplified coupling of surface plasmon resonance (SPR) immuno-biosensing with ambient ionization mass spectrometry (MS) was developed. It combines two orthogonal analysis techniques: the biosensing capability of SPR and the chemical identification power of high resolution MS. As a proof-of-principle, deoxynivalenol (DON), an important mycotoxin, was captured using an SPR gold chip containing an antifouling layer and monoclonal antibodies against the toxin and, after washing, the chip could be taken out and analyzed by direct spray MS of the biosensor chip to confirm the identity of DON. Furthermore, cross-reacting conjugates of DON present in a naturally contaminated beer could be successfully identified, thus showing the potential of rapid identification of (un)expected cross-reacting molecules. 


\section{Introduction}

Surface plasmon resonance (SPR) is a powerful biosensing tool for the real-time detection of a wide range of molecules. Apart from that, SPR provides important information about the binding kinetics of a biorecognition event. However, SPR does not provide the chemical identity of the binding analyte. ${ }^{1}$ Monoclonal antibodies are used in SPR for the selective immuno capturing and detection of the analyte. Although antibodies are highly specific, conjugates of the analyte can sometimes cross-react with the antibodies. Such cross-reacting conjugates cannot be distinguished from the targeted analyte by SPR, and may lead to overestimation of the analyte concentration. Therefore, coupling of SPR with mass spectrometry (MS) would not only confirm the identity of the SPR-detected target analyte(s), but might also help to find any (un)expected crossreacting analytes. ${ }^{2}$ As the bioreagents used for SPR are not MS-compatible, initial elution-based SPR-MS methods involved on-line collection of the desorbed analyte on a pre-column, followed by sample clean-up and off-line transfer of the pre-column with the sample to an electrospray ionization (ESI) tandem MS system. ${ }^{3}$ This leads to sample losses, which complicates the MS identification due to the minute amounts present. More sophisticated elution-based SPR-MS couplings allow real on-line MS analysis of analytes, however, the interfacing can be rather complex and expensive. ${ }^{4-6}$ Alternatively, coupling of SPR and MS based on matrix-assisted laser desorption ionization (MALDI) allows direct analysis of the biosensor chip, ${ }^{7,8}$ but requires the addition of an excess of MALDI matrix. The abundant matrix (cluster) ions can easily obscure the ions of small molecules ( $<700$ Da) present at sub-nanogram levels, hence most of the SPR-MALDI MS studies focus on the identification of peptides and proteins.

Ambient ionization methods for mass spectrometry, such as direct analysis in real time $(D A R T)^{9}$ and desorption electrospray ionization (DESI), ${ }^{10,11}$ have gained significant attention in the past decade as analyses can be performed at room temperature, under atmospheric conditions, often require minimal sample preparation and are suitable for small molecules. ${ }^{12}$ Ionization methods based on direct spray, ${ }^{13}$ where the sample is loaded onto a solid substrate (paper, metal, wood, glass, etc. $)^{14-16}$ followed by application of a high voltage (HV) to generate ions have become popular due to their simplicity. These methods rely on extraction/desorption of the analytes from the surface of the substrate using an organic solvent and, consequently, the overall selectivity is entirely dependent on the resolution of the mass analyzer. Recently, in an attempt to get rid of interfering components, coated blade spray was demonstrated in which solid-phase micro extraction (SPME) was coupled with a desorption electrospray ionization (DESI) source. ${ }^{17}$ As the analyte of interest was captured by the SPME coating, the method offered both 
sample enrichment and removal of other components using a washing step prior to MS analysis.

The aim of the present paper was the development of a simplified SPR-MS coupling. An SPR biochip coated with antibodies was used to selectively capture the analyte in an SPR apparatus. The SPR chip was taken out and following the application of a solvent and a high voltage, the analytes were desorbed and directly sprayed into a high- resolution MS (HRMS). In contrast to other direct MS approaches using affinity surfaces, such as, for example, surface-enhanced laser desorption/ionization (SELDI) ${ }^{18}$ and self-assembled monolayers laser desorption/ionization (SAMDI) ${ }^{19,20}$ the proposed concept combines two orthogonal analysis techniques. Moreover, the analysis of low molecular weight compounds was not obstructed by MALDI matrix ions.

A

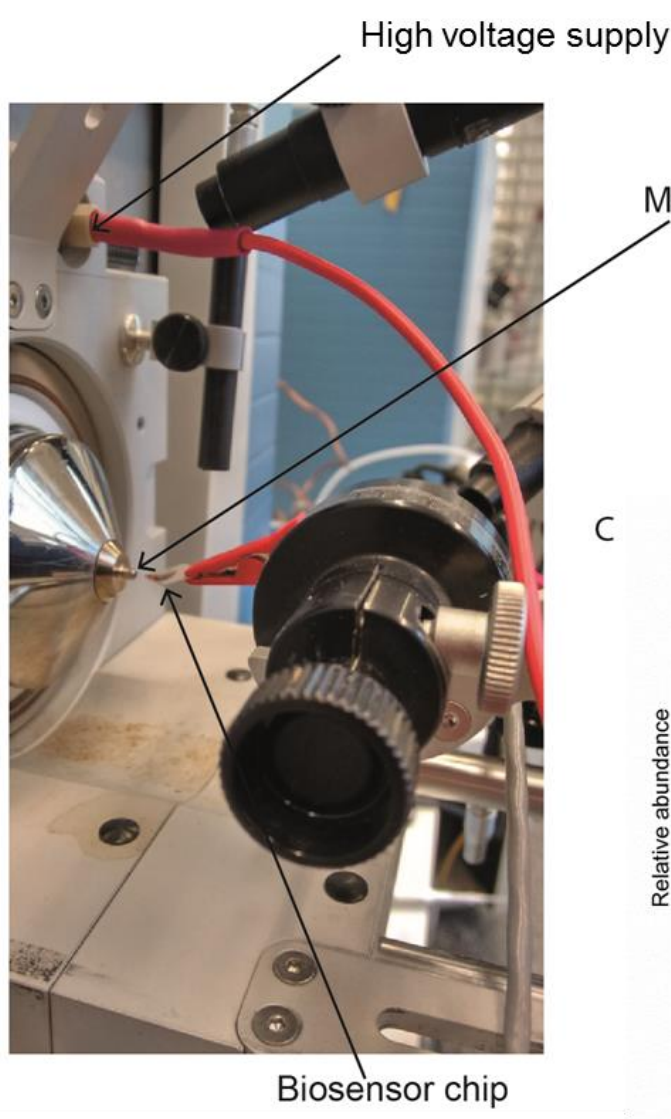

B
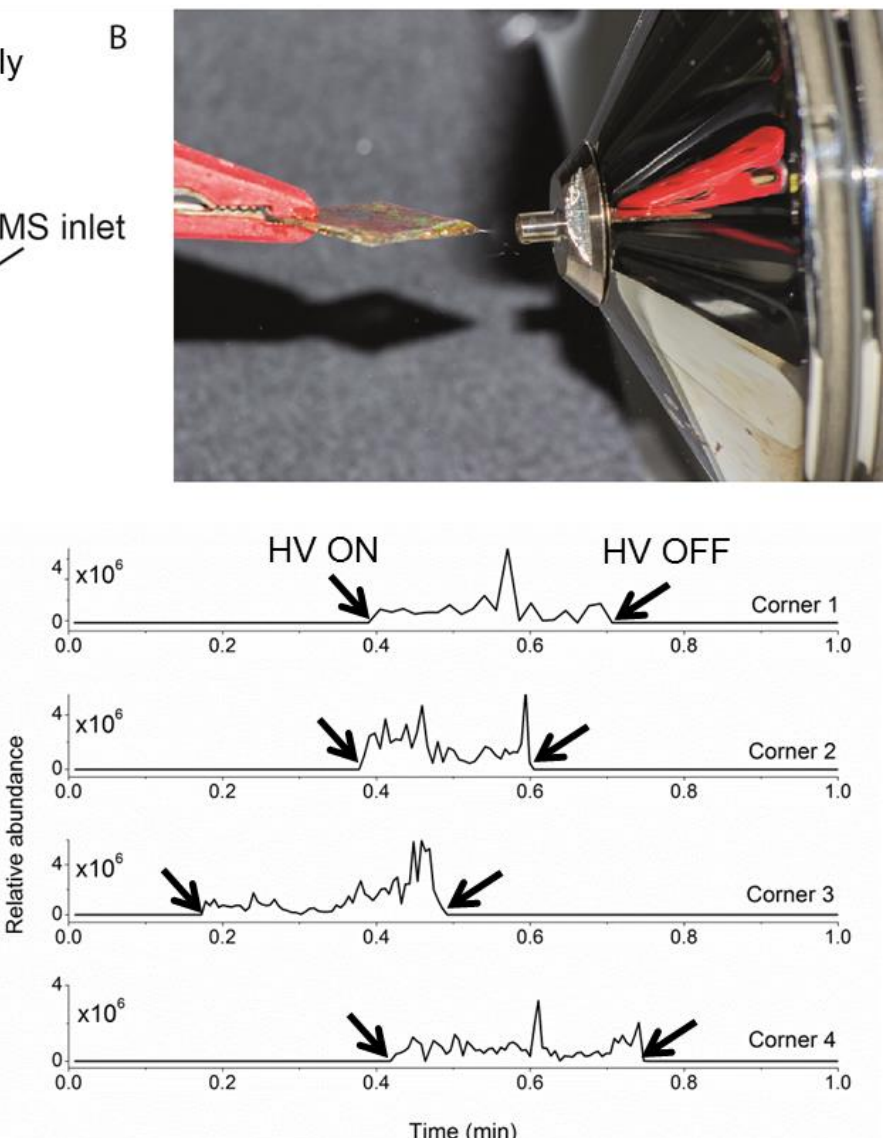

Figure 1. A) The setup used for Biochip Spray MS using a gold biosensor chip held in front of the MS inlet using an alligator clip, and B) Spray obtained after adding $10 \mu \mathrm{L}$ of methanol and applying a voltage of $5 \mathrm{kV}$. C) Extracted ion chronogram for $\mathrm{m} / \mathrm{z} 297.1333\left([\mathrm{DON}+\mathrm{H}]^{+}\right)$recorded in positive ion mode, as obtained from four different corners of a single $1 \mathrm{~cm}^{2}$ square carboxymethylated dextran (CMD) modified gold chip. $5 \mu \mathrm{L}$ of a $1 \mu \mathrm{g} / \mathrm{mL}$ DON solution in methanol were pipetted on each corner, allowed to dry and afterwards sprayed using methanol. The time points when the high voltage (HV) was turned on and off are indicated by arrows. 


\section{Experimental section}

Carboxymethylated dextran (CMD) coated flat gold chips were purchased from Xantec (Düsseldorf, Germany). Nanostructured gold chips were purchased from Plasmore Srl. (Ispra, Italy) and were further modified with CMD by Xantec. SPR measurements on flat gold chips were performed using a Biacore 3000 and iSPR measurements on nanostructured gold chips using a prototype portable imaging nanoplasmonics instrument (Plasmore Srl., Italy). ${ }^{21,22}$ Ethanolamine hydrochloride, 1-ethyl-3-(3dimethylaminopropyl) carbodiimide hydrochloride (EDC) and $\mathrm{N}$-hydroxysuccinimide (NHS) were purchased from Sigma Aldrich (Zwijndrecht, The Netherlands). Methanol was purchased from VWR (Amsterdam, the Netherlands). HBS-EP buffer (0.01 M HEPES pH 7.4, $0.15 \mathrm{M} \mathrm{NaCl}, 3 \mathrm{mM}$ EDTA, $0.005 \% \mathrm{v} / \mathrm{v}$ Surfactant P20) was purchased from GE Healthcare (Diegem, Belgium). MilliQ water $(18.3 \mathrm{M} \Omega \cdot \mathrm{cm}$ resistivity) was obtained using a Merck (Amsterdam, The Netherlands) water purification system.

The immobilization of antibodies on the CMD-modified gold chips for SPR was performed manually on the bench: the entire chip was activated using $50 \mu \mathrm{L}$ of a $1: 1$ mixture of $0.1 \mathrm{M} \mathrm{NHS}$ and $0.4 \mathrm{M}$ EDC for $30 \mathrm{~min}$, followed by washing with water and drying with nitrogen. Next, $50 \mu \mathrm{L}$ of antibody solution $(50 \mu \mathrm{g} / \mathrm{mL}$ in $10 \mathrm{mM}$ acetate buffer $\mathrm{pH}$ 4.5) were added to the activated chip. The chip was kept in an air-tight container for $2 \mathrm{~h}$ to avoid evaporation and drying out of the solution. Following the incubation, the chips were washed with water and dried with nitrogen. Unreacted groups were blocked with ethanolamine for $30 \mathrm{~min}$. Then the chips were rinsed with MilliQ water and dried with nitrogen. The chips were stored at 4-8 ${ }^{\circ} \mathrm{C}$ until use. The CMD chips (with or without antibodies) were mounted on the SPR flow cell and flushed with HBS-EP buffer for 5 min to allow swelling of the hydrogel. This was followed by injection of $50 \mu \mathrm{L}$ of sample (blank or spiked buffer or beer). The chip was then flushed with HBS-EP ( $5 \mathrm{~min}$ ) followed by water (5 min). Finally, the chip was unmounted from the SPR flow cell, dried with nitrogen and mounted in the Biochip Spray MS setup.

The chip was clamped by an alligator clip, which was part of a modified DESI ion source (Prosolia, USA) equipped with a rotational and $x-y-z$ positioner, and was directly connected to the HV supply of the ion source (Figure $1 A$ ). The square chip was positioned at an angle $\left(2-4^{\circ}\right)$ with one of the corners pointing downwards towards the MS inlet and at a distance of 4-6 mm (Figure 1B). $5 \mu \mathrm{L}$ of spray solvent was added using an Eppendorf pipette $(0.5-10 \mu \mathrm{L})$. After a waiting time of $30 \mathrm{~s}$, a voltage of $5 \mathrm{kV}$ was applied. All MS analyses were performed with a model Q-Exactive Focus quadrupole orbitrap high resolution MS (Thermo Fisher Scientific) to obtain full-scan positive ion measurements with a scan range of $m / z$ 150.0-1000.0, a mass resolution of 70,000 , a maximum injection time of $100 \mathrm{~ms}$ and a scan rate of $1 \mathrm{~Hz}$. The capillary temperature was $250^{\circ} \mathrm{C}$ 
and the S-lens RF level was 50. Caution notice: high voltages are involved! Prior to adding the spray solvent, always check that the voltage is actually $0 \mathrm{~V}$ and only switch it on when nobody is near the tip. The setup should not be touched during the experiment. Thermo Scientific Xcalibur software was used for data acquisition and processing. The intensity of the ions with $\mathrm{m} / \mathrm{z}$ values within $\pm 5 \mathrm{ppm}$ of the theoretical $\mathrm{m} / \mathrm{z}$ are shown in the extracted ion chronogram (EIC).

\section{Results and discussion}

Although any analyte-antibody pair could have been selected to show the concept, this study uses a low molecular weight mycotoxin, deoxynivalenol (DON), and a corresponding monoclonal antibody (anti-DON), due to its societal relevance. Deoxynivalenol is a secondary metabolite of fungi and is commonly found in several foods such as nuts, cereals, coffee, oil seeds and fruits, as well as in beverages and animal feed. ${ }^{23-25}$ Due to the thermal stability and relatively good water solubility, DON can be carried-over from a contaminated starting ingredient like barley to malt and finally into beer. ${ }^{26,27}$ Firstly, to demonstrate the feasibility of direct spray from a gold surface for analysis of DON, $5 \mu \mathrm{L}$ of $1 \mu \mathrm{g} / \mathrm{mL}$ (5000 pg) DON was placed in one corner of a CMD-modified gold biosensor chip (without any biorecognition) after which the solvent was allowed to evaporate. Then, $10 \mu \mathrm{L}$ of methanol was placed on the same corner of the chip followed by application of a high voltage to generate the spray. The voltage and distance between the chip and the MS inlet were optimized in order to obtain a stable spray without electric discharge. Optimal settings were $5 \mathrm{kV}$ at a distance of 4-6 mm between the chip and the MS. Although shorter distances than $4 \mathrm{~mm}$ required a lower voltage for spraying, electric discharge was occasionally seen whereas larger distances (>6 mm) in combination with higher voltages led to either an unstable spray or no spray at all. Furthermore, the angle was adjusted such that the added methanol neither spread over the chip nor started to drip off from the chip but rather remained at one corner. Under these optimized conditions, a stable spray for about 10-20 s was generated. Ions

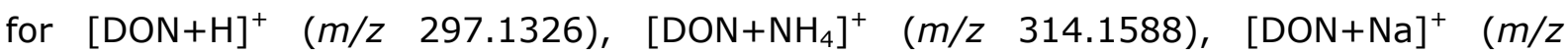
$319.1150)$ and $[D O N+K]^{+}(m / z \quad 335.0882)$ were observed in positive ion mode (see Figure $\mathrm{S} 1 \mathrm{~A})$. Although the $[\mathrm{DON}+\mathrm{Na}]^{+}$ion showed the highest intensity, $[\mathrm{DON}+\mathrm{H}]^{+}$was chosen for identification as an unknown positive ion with $\mathrm{m} / \mathrm{z} 319.1150$ (Figure S1B) was also seen in blank measurements (methanol spraying solvent only). As the unidentified interferent ion had, within experimental mass accuracy error, the same exact mass as $[\mathrm{DON}+\mathrm{Na}]^{+}$, using a higher resolution mass analyzer was not an option to resolve the $[\mathrm{DON}+\mathrm{Na}]^{+}$and the interferent ions. Measurements were performed in negative ion mode as well but positive ionization was chosen because the intensities of the ions were 
at least an order of magnitude higher. When four corners of the $1 \times 1 \mathrm{~cm}$ chips were subsequently used, quite reproducible average signal intensities (total area of the signal (in arbitrary units) divided by the time of signal duration (in min)) of $8.1 \times 10^{7}, 2.7 \times 10^{8}$, $1.5 \times 10^{8}$ and $1.1 \times 10^{8}$, respectively, were obtained (Figure $1 \mathrm{C}$ ). The comparable results obtained for the four different corners suggest that a single chip can be analysed four times, for example serving the purpose of replicate analyses or even multiplexing by immobilizing four different biorecognition elements in each corner of the square chip. No physical damage (scratching) was observed on the side used earlier for clamping as the CMD modified chips are quite scratch-resistant. Next, serial dilutions of DON were analyzed (5000 pg, $500 \mathrm{pg}, 50 \mathrm{pg}$ and $5 \mathrm{pg}$ ) yielding a decrease of an order of magnitude of average signal intensities at each dilution step $\left(10^{8}\right.$ for $5000 \mathrm{pg}, 10^{7}$ for 500 $\mathrm{pg}$ and $10^{6}$ for $50 \mathrm{pg}$ ). No signal for DON could be observed when only $5 \mathrm{pg}$ of the toxin was spiked onto the chip. Washing of the CMD gold chip (after spiking with DON and drying) with HBS-EP and water was performed to check if any DON could be nonspecifically adsorbed to the chip surface without antibodies. Even when spiked at the highest level (5000 pg), no ions for DON were observed following washing.

For the Biochip Spray measurements, anti-DON was covalently immobilized on gold chips coated with carboxymethylated dextran. This was followed by introduction of buffer or beer containing the toxins $(10 \mu \mathrm{g} / \mathrm{mL}$ of DON) and washing of the chips (HBSEP followed by water), both performed in the SPR instrument. Washing of the chip with buffer (HBS-EP) helped to get rid of any non-specifically adsorbed mycotoxin or sample components while the washing with water removed buffer salts, making the chip suitable for direct spray MS experiments. The chip was then unmounted from the SPR flow cell, dried with nitrogen, and subsequently positioned in front of the MS inlet capillary. The choice of solvent is an additional parameter as it must not only be efficient for creating a stable electrospray, but should also be able to disrupt the interaction of antibodies (antiDON) with the mycotoxins (DON). Like in direct spray, $100 \%$ methanol gave a reproducible and stable spray. Using a lower concentration of methanol (90\% methanol; $10 \%$ water) was not feasible as it often required higher voltages ( $>6 \mathrm{kV}$ ) and the spray was not reproducible. In earlier SPR studies, acidic $(10 \mathrm{mM} \mathrm{HCl})$ and basic conditions (20 $\mathrm{mM} \mathrm{NaOH}$ ) were found to be suitable for disrupting the interaction of antibody and antigen. ${ }^{28,29}$ However, these SPR chip regenerants are not compatible with MS experiments and must be replaced with, for example, formic acid or ammonium hydroxide. So the addition of $1-2 \%$ of formic acid or ammonium hydroxide to methanol was tested as well, but the best disruption and direct spray performance was still obtained with $100 \%$ methanol. As can be seen from Figure $2 \mathrm{~A}$ and $2 \mathrm{~B}$, ions for DON could be observed in both spiked buffer and spiked beer. In the Biochip Spray experiment, $10 \mu \mathrm{g} / \mathrm{mL}$ of toxins was used to ensure saturation of the available binding 
sites of the antibodies on the surface as observed in the calibration curves from SPR experiments (Figure S2B). But only a limited amount can be captured and thus be later detected by Biochip Spray MS. When comparing the obtained average signal intensity $\left(10^{6}\right)$ with the spiked solution experiments described above, approximately $\sim 50 \mathrm{pg}$ of DON, i.e., in the range expected based on the amount of anti-DON present according to SPR, was immuno-captured and subsequently analyzed by Biochip Spray MS. To ensure that desorption was complete, the chip after having been used for the Biochip Spray MS experiment (no signals were observed in the second spray) was placed in a methanol solution $(200 \mu \mathrm{L})$ for 5 min with occasional shaking. The resulting extract was evaporated to dryness, re-dissolved in $5 \mu \mathrm{L}$ of methanol and analyzed by ESI-MS. The absence of any ions from DON confirmed the complete desorption. Another parameter that has been suggested for optimization in the literature is the waiting time before starting the spray. ${ }^{17}$ Therefore, 0,30 , and $60 \mathrm{sec}$ of waiting time were tested to see if there was any effect on the amount of analyte desorbed. Insignificant differences were observed between the different waiting times, so $30 \mathrm{~s}$ of waiting time was chosen to ensure sufficient and controlled timing between adding the methanol solution and applying the voltage. To confirm the selectivity of the developed method, two negative control experiments were performed. The first negative control was a chip containing anti-DON but incubated in blank beer: the absence of DON ions was confirmed by Biochip Spray MS. Another control experiment was performed by immobilizing an antibody against another mycotoxin, fumonisin (anti-FB ${ }_{1}$ ) elsewhere on the same chip. Based on previous research, ${ }^{28}$ anti-FB ${ }_{1}$ does not cross-react with DON. After introduction of beer spiked with DON and the standard washing procedure, high voltage was applied to the chip. A change indicating the onset of the spray was seen in the total ion chronogram (Figure $\mathrm{S} 3 \mathrm{~A}$ ) but no ions for DON were observed (zero signal in the extracted ion chronogram, Figure S3B). The above mentioned experiments serve as true negative controls for two reasons. Firstly, they could be performed on another corner of the same chip, the first half of which was used for immobilization of anti-DON. Secondly, the anti-FB ${ }_{1}$ used is also an IgG and would account for any non-specific adsorption that could occur during the experiment. Re-use of the SPR sensor chip is still a big challenge in SPR-MS as the conditions used for desorption and ionization involve organic solvents that not only disrupt the antibody-antigen interaction but also unfold the immobilized antibodies. ${ }^{7}$ Indeed, we observed similar problems: the chips coated with antibodies could only be used for SPR biosensing once. Upon re-incubation of the same chip in a solution containing the analyte, no DON signal could be obtained. However, the proposed four corner approach for replicate analysis, multiplexing or negative control experiments offers a reasonable compromise between reliable results and economy of use. 


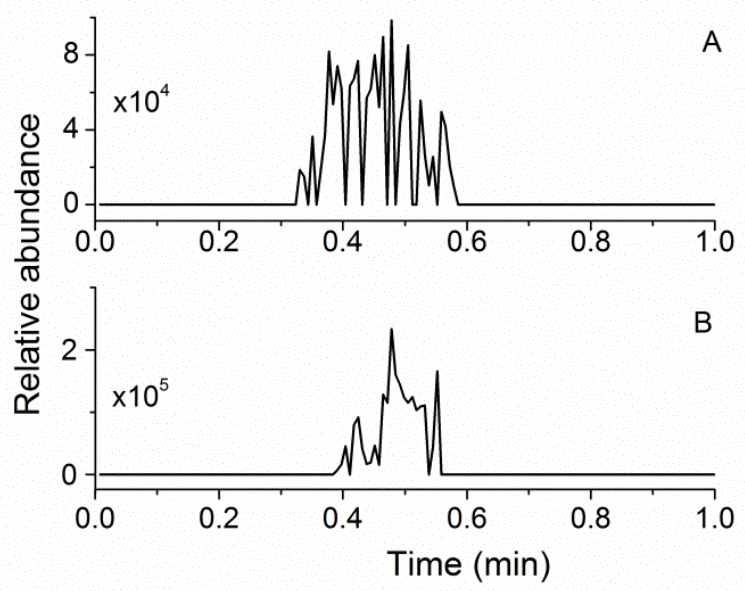

Figure 2. Extracted ion chronogram for $\mathrm{m} / \mathrm{z} 297.1333\left([\mathrm{DON}+\mathrm{H}]^{+}\right)$recorded in positive mode obtained from a CMD-modified gold chip with immobilized anti-DON, flushed in the iSPR flow cell with A) spiked buffer containing $10 \mu \mathrm{g} / \mathrm{mL}$ of DON and B) spiked beer containing $10 \mu \mathrm{g} / \mathrm{mL}$ of DON, followed by washing with buffer and water and transfer to the Biochip Spray MS set-up.

Finally, to demonstrate the real-life application of the newly developed method, a naturally contaminated beer sample containing $2760 \pm 95 \mathrm{ng} / \mathrm{mL}$ of DON plus $3883 \pm$ $137 \mathrm{ng} / \mathrm{mL}$ of deoxynivalenol-3-glucoside (D3G) according to LC-MS/MS ${ }^{30}$, or $5290 \pm$ $370 \mathrm{ng} / \mathrm{mL}$ according to SPR (DON plus conjugates) was used. ${ }^{29}$ Large molecular weight analytes such as proteins, yield high signals in SPR sensing with an antibody biochip and may be analyzed in a direct SPR assay (Figure S2A + S4A) without signal enhancer. Subsequently, the binding molecules may be identified in a direct manner by MS using either MALDI ${ }^{7,8}$ or following elution ${ }^{3}$ using ESI MS or potentially by Biochip Spray MS (Figure S4A). However, low molecular weight analytes, such as DON, are much more challenging to detect in label-free biosensing approaches such as SPR: in a direct SPR mode with an antibody biochip a low and noisy signal (Figure S2A, solid line, Biacore) or no signal (iSPR) will be obtained, unless the assay is changed into a competition format using a signal enhancer such as DON-OVA (Figure S2A, dashed line). Moreover, direct MS identification of low-molecular weight binding molecules would be challenging as well due to, e.g., matrix interference in MALDI MS and ion suppression and interference from residual assay reagents in ESI MS. Therefore, we performed SPR biosensing of DON in the contaminated beer sample in two different modes: (i) a competitive direct mode, using an anti-DON biochip and the addition of DON-OVA to the beer sample as a signal enhancer (Figure S2B + S4B), and (ii) a competitive indirect mode using an immobilized DON biochip and the beer sample mixed with anti-DON (Figure S2D + S4C). In both SPR modes almost full signal inhibition was observed due to the presence of DON and conjugates in the contaminated beer (Figure S2C and S2E). Please note that as for any 
large-scale routine SPR analysis of small molecules, a competitive indirect mode using an immobilized DON biochip (Figure S2D + S4C) would be the method of choice since such a chip will show extreme durability and, in the present case, could be used more than 400 times. ${ }^{29}$ Obviously, an immobilized DON chip would not directly trap the low molecular weight ligands for subsequent chemical identification by MS. As a way out, a so-called 'recovery-chip' approach was applied as demonstrated previously for the coupling of inhibition SPR with nanoLC MS. ${ }^{4}$ Such a recovery chip is identical to the SPR screening chip but the ligand binding interaction is reversed, i.e., immobilized antibodies on the chip to trap sufficient numbers of analyte molecules for chemical identification (Figure S4D). Thus the contaminated beer sample was re-injected into the SPR apparatus but now onto a chip containing the anti-DON followed by washing with HBS-EP and water (Figure S4D). The chip was then taken out of the SPR instrument, dried with nitrogen and analyzed using the Biochip Spray MS (Figure 3). In addition to the expected ions for DON, ions of D3G $\left(m / z 481.1680,[\mathrm{D} 3 \mathrm{G}+\mathrm{Na}]^{+}\right)$and acetyl DON (ADON, $\mathrm{m} / z$ 339.1438, $[\mathrm{ADON}+\mathrm{H}]^{+}$) were observed. Both $\mathrm{D} 3 \mathrm{G}$ and $\mathrm{ADON}$ are conjugates of DON known to cross-react with anti-DON. ${ }^{28}$ No ions from nivalenol (NIV) were visible and this is in-line with our previous SPR research, in which no cross-reactivity was observed. ${ }^{28}$

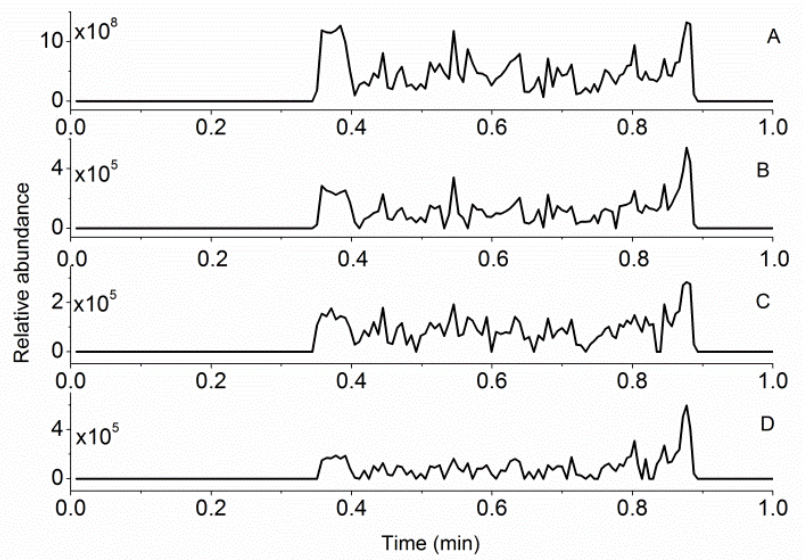

Figure 3. A) Total ion chronogram along with the extracted ion chronogram for $m / z$ (B) 297.1333 $\left([\mathrm{DON}+\mathrm{H}]^{+}\right),(\mathrm{C}) 481.1680\left([\mathrm{D} 3 \mathrm{G}+\mathrm{Na}]^{+}\right),(\mathrm{D}) 339.1438\left([\mathrm{ADON}+\mathrm{H}]^{+}\right)$, recorded in positive ion mode obtained from a CMD-modified gold chip with immobilized anti-DON. The chip was loaded in an iSPR apparatus and naturally contaminated beer was injected followed by washing with buffer and water and transfer to the Biochip Spray MS set-up.

To conclude, a simplified coupling of SPR with MS through direct Biochip Spray was developed that allows chemical identification of low molecular weight analytes in SPR ligand binding assays. The technique is based on selective capturing of a target analyte on an SPR biosensor chip containing antibodies (or any other biorecognition element), followed by identification of the analyte as well as any (un)expected cross-reacting 
conjugates using ambient ionization MS directly from the gold SPR chip. The method may be applied for those samples, which give a response in either indirect or direct SPR biosensor screening modes (fair to say that not many low molecular weight analytes will yield a significant response in the direct SPR mode). The aforementioned method is, in principle, generic, and could be applied to any MS-amenable analyte provided that antibodies are available and that they can be immobilized on an SPR chip. In this work, the antibodies were immobilized via the amine group and thus randomly oriented on the surface. Approaches for oriented immobilization of antibodies could be explored for better antigen binding and thus stronger signals in the MS. A 4-plex imaging SPR (iSPR) approach in which each corner of the biochip contains a different capturing antibody with the corresponding analyte that can be subsequently identified by Biochip Spray MS can be envisaged.

\section{Acknowledgments}

We would like to thank $\mathrm{Dr}$ Milena Zachariasova (Institute of Chemical Technology, Prague, CZ) for the contaminated beer samples, and $\mathrm{Dr}$ Wilco Duvivier (RIKILT, Wageningen, NL) and Ing. Frank Claassen (Wageningen University, NL) for technical assistance. This research received funding from the Netherlands Organization for Scientific Research (NWO) in the framework of the Technology Area COAST (project nr 053.21.107) with WU, VU Amsterdam, RIKILT, Heineken, Synthon, Technex, EuroProxima, Waterproef as partners and Plasmore and Bionavis as associated partners. 


\section{References}

1. Homola, J. Surface plasmon resonance sensors for detection of chemical and biological species. Chem. Rev. 2008, 108, 462-493.

2. Stigter, E. C. A.; de Jong, G. J.; van Bennekom, W. P. Coupling surface-plasmon resonance and mass spectrometry to quantify and to identify ligands. Trends Anal. Chem. 2013, 45, 107-120.

3. Natsume, T.; Nakayama, H.; Jansson, Ö.; Isobe, T.; Takio, K.; Mikoshiba, K. Combination of biomolecular interaction analysis and mass spectrometric amino acid sequencing. Anal. Chem. 2000, 72, 4193-4198.

4. Marchesini, G. R.; Buijs, J.; Haasnoot, W.; Hooijerink, D.; Jansson, Ö.; Nielen, M. W. F. Nanoscale affinity chip interface for coupling inhibition SPR immunosensor screening with nano-LC TOF MS. Anal. Chem. 2008, 80, 1159-1168.

5. Zhang, Y.; Li, X.; Nie, H.; Yang, L.; Li, Z.; Bai, Y.; Niu, L.; Song, D.; Liu, H. Interface for online coupling of surface plasmon resonance to direct analysis in real time mass spectrometry. Anal. Chem. 2015, 87, 6505-6509.

6. Zhang, Y.; Xu, S.; Wen, L.; Bai, Y.; Niu, L.; Song, D.; Liu, H. A dielectric barrier discharge ionization based interface for online coupling surface plasmon resonance with mass spectrometry. Analyst 2016, 141, 3343-3348.

7. Krone, J. R.; Nelson, R. W.; Dogruel, D.; Williams, P.; Granzow, R. BIA/MS: Interfacing biomolecular interaction analysis with mass spectrometry. Anal. Biochem. 1997, 244, 124132.

8. Urban, P. L.; Amantonico, A.; Zenobi, R. Lab-on-a-plate: Extending the functionality of MALDI-MS and LDI-MS targets. Mass Spectrom. Rev. 2011, 30, 435-478.

9. Cody, R. B.; Laramée, J. A.; Durst, H. D. Versatile new ion source for the analysis of materials in open air under ambient conditions. Anal. Chem. 2005, 77, 2297-2302.

10. Takáts, Z.; Wiseman, J. M.; Gologan, B.; Cooks, R. G. Mass spectrometry sampling under ambient conditions with desorption electrospray ionization. Science 2004, 306, 471-473.

11. Nielen, M. W. F.; Hooijerink, H.; Zomer, P.; Mol, J. G. J. Desorption electrospray ionization mass spectrometry in the analysis of chemical food contaminants in food. Trends Anal. Chem. 2011, 30, 165-180.

12. Manova, R. K.; Joshi, S.; Debrassi, A.; Bhairamadgi, N. S.; Roeven, E.; Gagnon, J.; Tahir, M. N.; Claassen, F. W.; Scheres, L. M. W.; Wennekes, T.; Schroën, K.; van Beek, T. A.; Zuilhof, H.; Nielen, M. W. F. Ambient surface analysis of organic monolayers using direct analysis in real time orbitrap mass spectrometry. Anal. Chem. 2014, 86, 2403-2411.

13. Klampfl, C. W.; Himmelsbach, M. Direct ionization methods in mass spectrometry: An overview. Anal. Chim. Acta 2015, 890, 44-59.

14. Hu, B.; So, P.-K.; Yao, Z.-P. Electrospray ionization with aluminum foil: A versatile mass spectrometric technique. Anal. Chim. Acta 2014, 817, 1-8.

15. Jiang, J.; Zhang, H.; Li, M.; Dulay, M. T.; Ingram, A. J.; Li, N.; You, H.; Zare, R. N. Droplet spray ionization from a glass microscope slide: Real-time monitoring of ethylene polymerization. Anal. Chem. 2015, 87, 8057-8062.

16. Paine, M. R. L.; Barker, P. J.; Blanksby, S. J. Paint spray mass spectrometry for the detection of additives from polymers on conducting surfaces. Mass Spectrom. Lett. 2012, 3, 25-28.

17. Gómez-Ríos, G. A.; Pawliszyn, J. Development of coated blade spray ionization mass spectrometry for the quantitation of target analytes present in complex matrices. Angew. Chem. Int. Ed. 2014, 53, 14503-14507.

18. Seibert, V.; Wiesner, A.; Buschmann, T.; Meuer, J. Surface-enhanced laser desorption

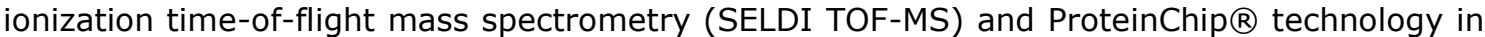
proteomics research. Pathol. Res. Pract. 2004, 200, 83-94.

19. Patrie, S. M.; Mrksich, M. Self-assembled monolayers for MALDI-TOF mass spectrometry for immunoassays of human protein antigens. Anal. Chem. 2007, 79, 5878-5887.

20. Mrksich, M. Mass spectrometry of self-assembled monolayers: A new tool for molecular surface science. ACS nano 2008, 2, 7-18.

21. Valsesia, A.; Marabelli, F.; Giudicatti, S.; Marchesini, G. R.; Rossi, F.; Colpo, P. SPR sensor device with nanostructure. World Patent 2013, WO 2013/007448 A1.

22. Bottazzi, B.; Fornasari, L.; Frangolho, A.; Giudicatti, S.; Mantovani, A.; Marabelli, F.; Marchesini, G.; Pellacani, P.; Therisod, R.; Valsesia, A. Multiplexed label-free optical biosensor for medical diagnostics. J. Biomed. Opt. 2014, 19, 017006-017010.

23. Kostelanska, M.; Hajslova, J.; Zachariasova, M.; Malachova, A.; Kalachova, K.; Poustka, J.; Fiala, J.; Scott, P. M.; Berthiller, F.; Krska, R. Occurrence of deoxynivalenol and its major 
conjugate, deoxynivalenol-3-glucoside, in beer and some brewing intermediates. J. Agric. Food Chem. 2009, 57, 3187-3194.

24. Papadopoulou-Bouraoui, A.; Vrabcheva, T.; Valzacchi, S.; Stroka, J.; Anklam, E. Screening survey of deoxynivalenol in beer from the European market by an enzyme-linked immunosorbent assay. Food Addit. Contam. 2004, 21, 607-617.

25. Zachariasova, M.; Hajslova, J.; Kostelanska, M.; Poustka, J.; Krplova, A.; Cuhra, P.; Hochel, I. Deoxynivalenol and its conjugates in beer: A critical assessment of data obtained by enzyme-linked immunosorbent assay and liquid chromatography coupled to tandem mass spectrometry. Anal. Chim. Acta 2008, 625, 77-86.

26. Inoue, T.; Nagatomi, Y.; Uyama, A.; Mochizuki, N. Fate of mycotoxins during beer brewing and fermentation. Biosci. Biotechnol. Biochem. 2013, 77, 1410-1415.

27. Lancova, K.; Hajslova, J.; Poustka, J.; Krplova, A.; Zachariasova, M.; Dostalek, P.; Sachambula, L. Transfer of Fusarium mycotoxins and 'masked' deoxynivalenol (deoxynivalenol-3-glucoside) from field barley through malt to beer. Food Addit. Contam. 2008, 25, 732-744.

28. Joshi, S.; Segarra-Fas, A.; Peters, J.; Zuilhof, H.; van Beek, T. A.; Nielen, M. W. F. Multiplex surface plasmon resonance biosensing and its transferability towards imaging nanoplasmonics for detection of mycotoxins in barley. Analyst 2016, 141, 1307-1318.

29. Joshi, S.; Annida, R. M.; Zuilhof, H.; Van Beek, T. A.; Nielen, M. W. F. Analysis of mycotoxins in beer using a portable nanostructured imaging surface plasmon resonance biosensor. J. Agric. Food Chem. 2016, 64, 8263-8271.

30. Zachariasova, M.; Vaclavikova, M.; Lacina, O.; Vaclavik, L.; Hajslova, J. Deoxynivalenol oligoglycosides: new "masked" Fusarium toxins occurring in malt, beer, and breadstuff. J. Agric. Food Chem. 2012, 60, 9280-9291. 


\section{Supporting information}
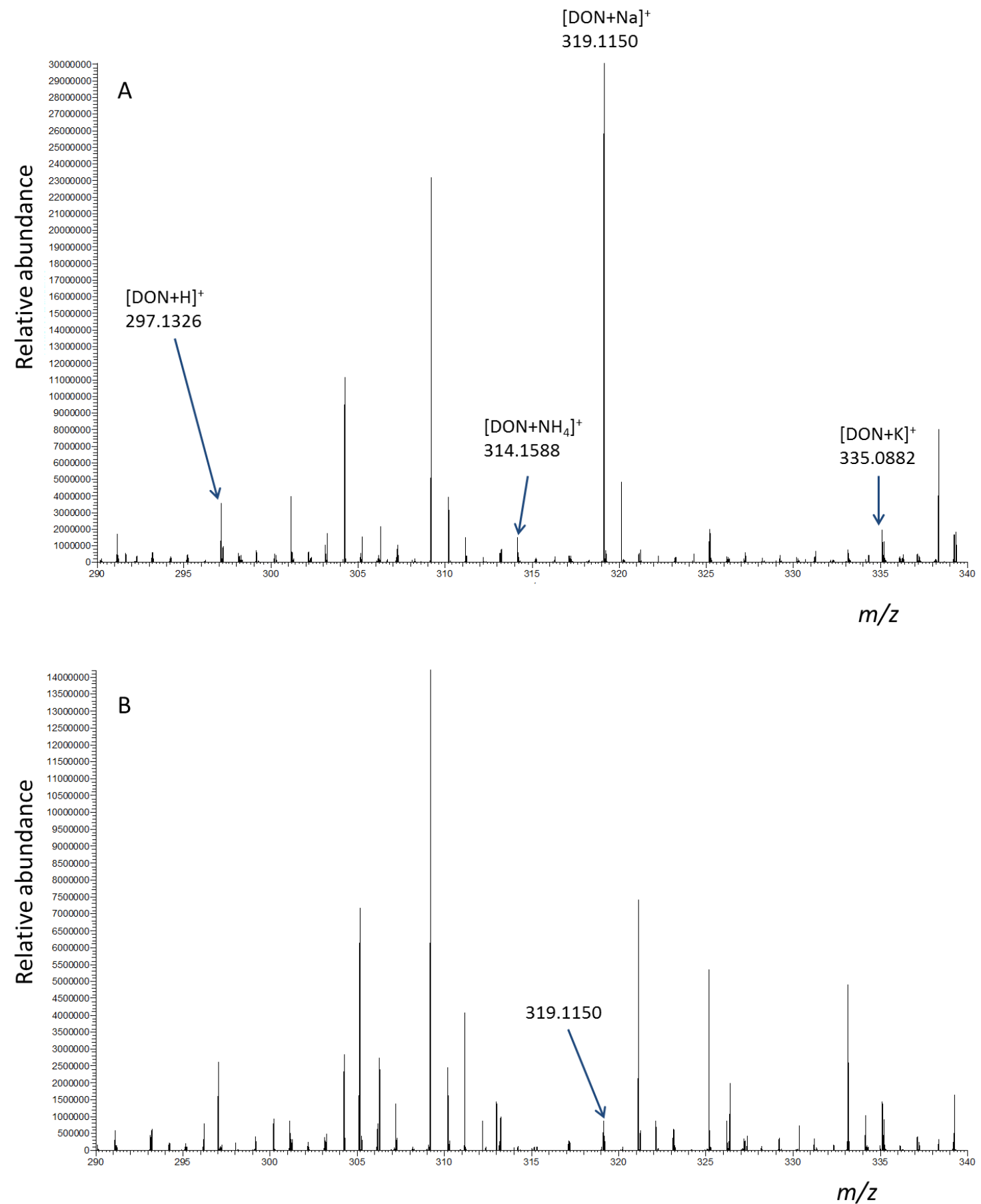

Figure S1. Chip Spray mass spectra recorded in positive ion mode from a CMD-modified gold chip spiked with A) $5 \mu \mathrm{L}$ of $1 \mu \mathrm{g} / \mathrm{mL}$ DON in methanol and B) $5 \mu \mathrm{L}$ of methanol, drying, and application of $5 \mu \mathrm{L}$ of methanol and $5 \mathrm{kV}$. In blank methanol an unknown species with $\mathrm{m} / z 319.1150$, i.e., the same $m / z$ as for [DON+Na] ${ }^{+}$was observed. 

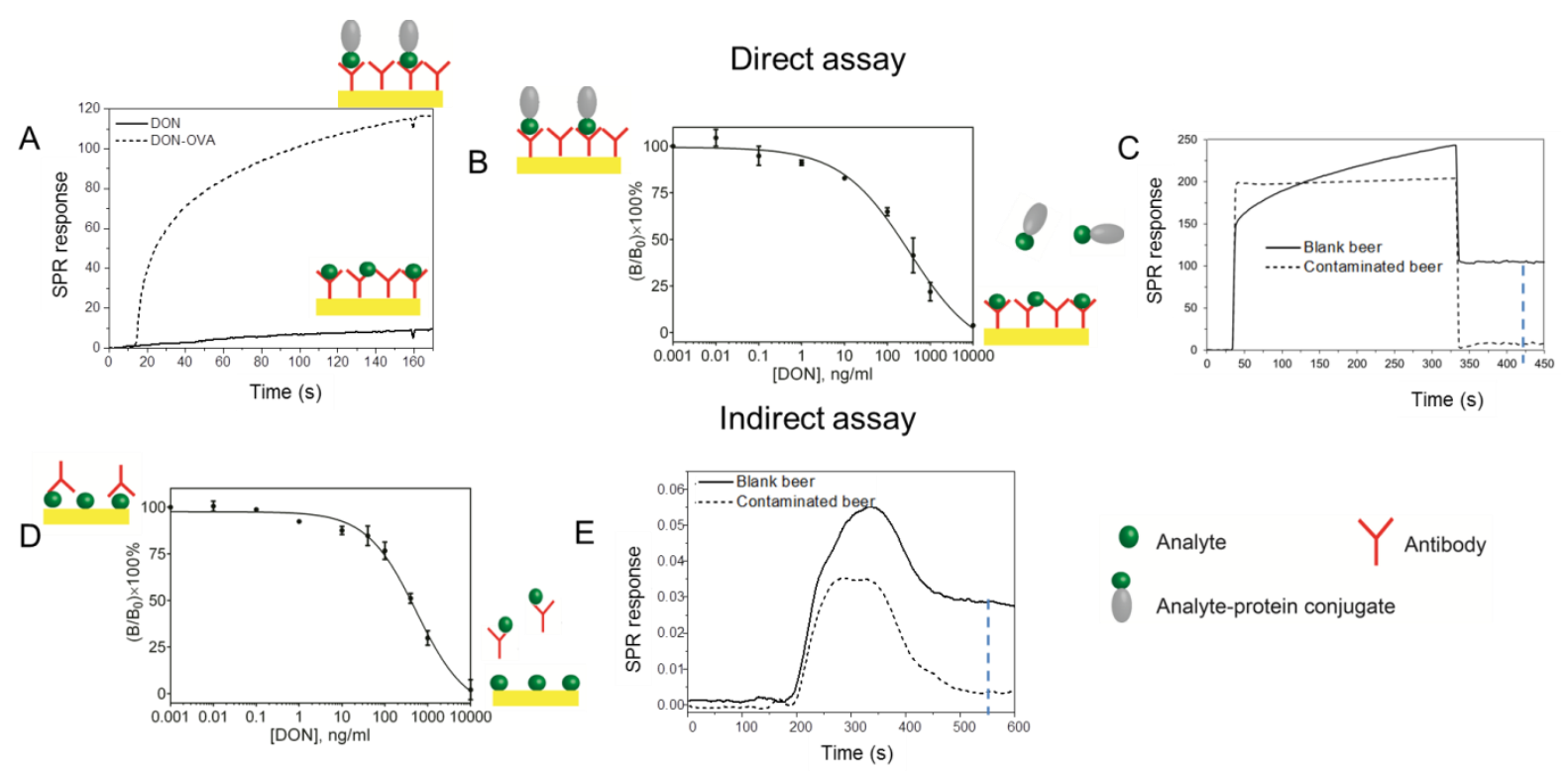

Figure S2. Different SPR assay modes. In a direct SPR assay, anti-DON is immobilized on the surface. The response obtained only for DON was weak ( $A$, solid line, Biacore) or absent (iSPR) thus requiring use of an ovalbumin conjugate of DON (DON-OVA) as a signal enhancer ( $A$, dashed line). B) The response of a fixed concentration of DON-OVA in competition with increasing concentration of DON (in sample) for the immobilized anti-DON is measured to construct a calibration curve. C) SPR response measured for blank beer and contaminated beer using the direct assay of B with DON-OVA as signal enhancer.

In an indirect SPR assay, DON is immobilized on the surface and the response of a fixed concentration of anti-DON with increasing concentrations of DON (in sample) in competition with the immobilized DON is measured (D). E) SPR response measured for blank beer and contaminated beer using the indirect assay of $D$.

A near-complete inhibition of the SPR response (taken at time points indicated by blue dashed lines in Figure S2C and E) is seen for the contaminated beer, indicating the presence of DON and/or cross-reacting conjugates. Note: the indirect assay is recommended for routine screening of large numbers of samples as the chips with immobilized DON are much more durable than the ones with immobilized antibodies (see also ref. 29 cited in the main text).

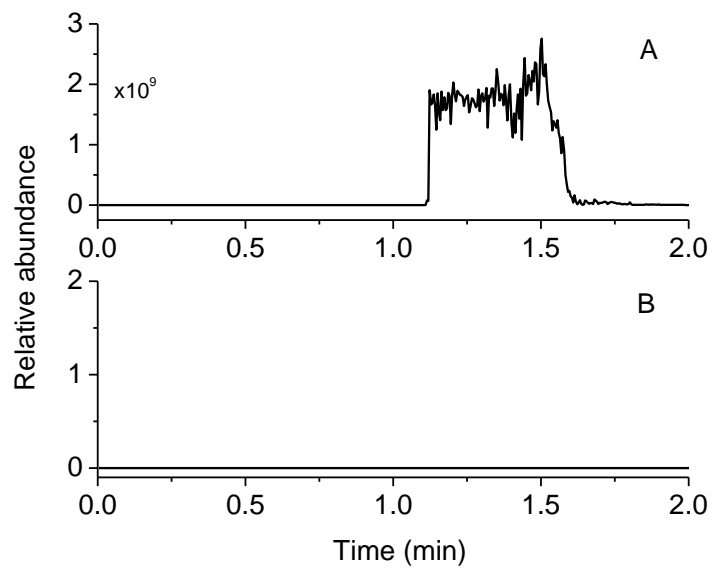

Figure S3. A) Total ion chronogram and B) extracted ion chronogram for $\mathrm{m} / z 297.1333$ $\left([\mathrm{DON}+\mathrm{H}]^{+}\right)$. Conditions: results obtained from a CMD-modified gold chip with immobilized anti-FB ${ }_{1}$ that was flushed in the flow cell of the iSPR with spiked beer (containing $10 \mu \mathrm{g} / \mathrm{mL}$ of DON), followed by washing of the anti-FB ${ }_{1}$ chip with buffer and water and transfer of the chip to the Biochip Spray MS set-up. 


\title{
For large molecules (e.g. proteins)
}

\author{
Sample \\ A \\ Chip with antibody \\ Direct SPR assay

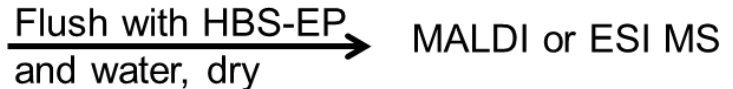 \\ Potentially DESI MS or \\ Biochip Spray MS
}

\section{For small molecules (e.g. DON)}

B

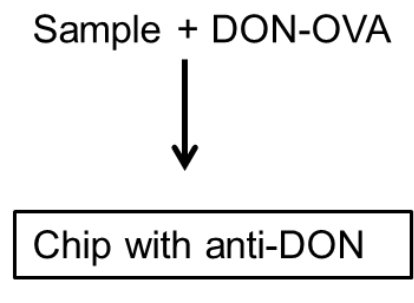

Direct SPR assay
C Sample + anti-DON

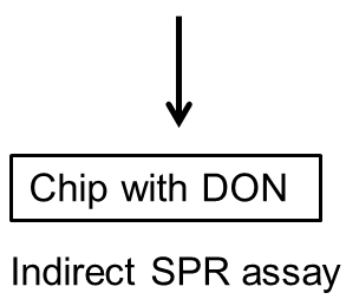

Positive or negative for DON

D Sample

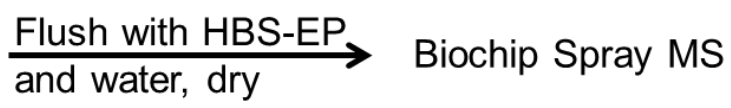

Confirmation of DON

Chip with anti-DON

Identification of conjugates

Recovery chip

Figure S4. Schematic representation of the workflow for SPR-MS. A) For large molecules such as proteins where the sample could be analysed using direct SPR assay (Figure S2A) without signal enhancer followed by various forms of MS analysis. For small molecules such as DON where the SPR biosensing can be performed in two modes, B) a competitive direct mode, using an anti-DON biochip and the addition of DON-OVA to the beer sample as a signal enhancer and C) a competitive indirect mode using an immobilized DON biochip and the beer sample mixed with anti-DON. D) A sample considered positive from the indirect competitive assay (Figure S2E) or the direct SPR assay (Figure S2C) is re-injected onto a 'recovery chip' containing anti-DON (like in the direct SPR assay format but without the competing DON-OVA conjugate being present, i.e., as in Figure S2A solid line), followed by Biochip Spray MS analysis. 


\section{Chapter 6}

General discussion and future perspectives 


\section{General Discussion}

Over the past decades, surface plasmon resonance has proven to be a powerful tool for biosensing applications. However, the size of conventional SPR instruments has limited their application in well-organized laboratory settings. Bringing the SPR instrument to the sample requires stringent miniaturization of SPR components, reduced weight and power consumption and a much simpler design. The prototype nanostructured iSPR instrument used in this thesis has been able to fulfill these criteria and was studied as a potential screening device for in-field and at-line applications. Development of such a prototype instrument comes with several challenges that will be discussed in the following sections with a reflection on the future perspectives of the technology.

\section{Quality control of iSPR chips}

The nanostructured chip, core to the iSPR technology, was recently developed and has been studied only to a limited extent. Therefore, as discussed in Chapter 2, the detailed characterization of the chip before and after surface modification is very important. Several techniques were used to obtain complementary information about the chips. In addition to the chemical characterizations (AFM, SEM, water contact angle, XPS and DART-HRMS), optical characterization of the chips is also essential as the optical properties influence the iSPR performance. Spectrophotometry was used for the characterization of the chips and served as a quality control tool. The visible and near IR (350-1000 nm) spectra of the chips were measured in reflectance mode upon addition of water. Another spectrum was recorded after replacing the water with $10 \%$ ethanol and the intensity of the reflected light with ethanol, relative to that of water, was used as the normalized intensity (Figure 1). Chips showing a change of +5 to $+10 \%$ at $850 \mathrm{~nm}$ and -5 to $-10 \%$ at $940 \mathrm{~nm}$ (Figure $1, \mathrm{~A}$ ) displayed the best sensitivities in terms of iSPR response obtained later for a fixed concentration of antibodies in the indirect mycotoxin assay. However, variations were observed (Figure 1, B) from batch to batch and sometimes even within batches. The chips that did not fulfill the above spectrophotometric criteria were also more sensitive to peeling off of the gold layer during sonication or to scratching during handling. Although the manufacturer tried to improve the durability by using an annealing step, this led to a decrease in the water contact angle. It was observed that chips with very low contact angles $\left(10^{\circ}-30^{\circ}\right)$ were not suitable for further surface modification using thiol chemistry. Comparing the PEG3500 modification of the chips with the higher contact angle $\left(60^{\circ}-80^{\circ}\right)$ (Figure $2 \mathrm{~A}$, right), with those having a lower contact angle $\left(10^{\circ}-30^{\circ}\right)$ (Figure $2 \mathrm{~B}$, right), showed that the latter contained less PEG (less signal in the corresponding C1s narrow scan). The survey spectra of the XPS revealed a significantly higher amount of silicon and oxygen in the 
case of the chips having a low contact angle (Figure 2B, left). That could be the reason for poor surface modification as less gold is available for modification. Therefore, good quality control based on a combination of optical, physical and surface chemical characterization was crucial for the reproducibility of the experiments presented in this thesis.

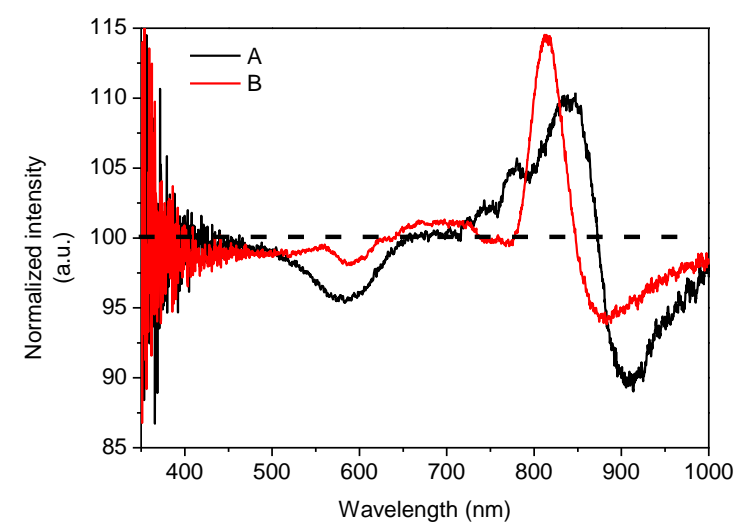

Figure 1. Differences in the normalized intensities of the reflected light of two different chips. The intensity of the reflected light is measured for $10 \%$ ethanol relative to water using the same chip. Chip A with a change of $+8 \%$ at $850 \mathrm{~nm}$ and $-6 \%$ at $940 \mathrm{~nm}$ satisfies the quality control demands, whereas Chip B with a change of $-1 \%$ at $850 \mathrm{~nm}$ and $-3 \%$ at $940 \mathrm{~nm}$ does not meet the requirements.
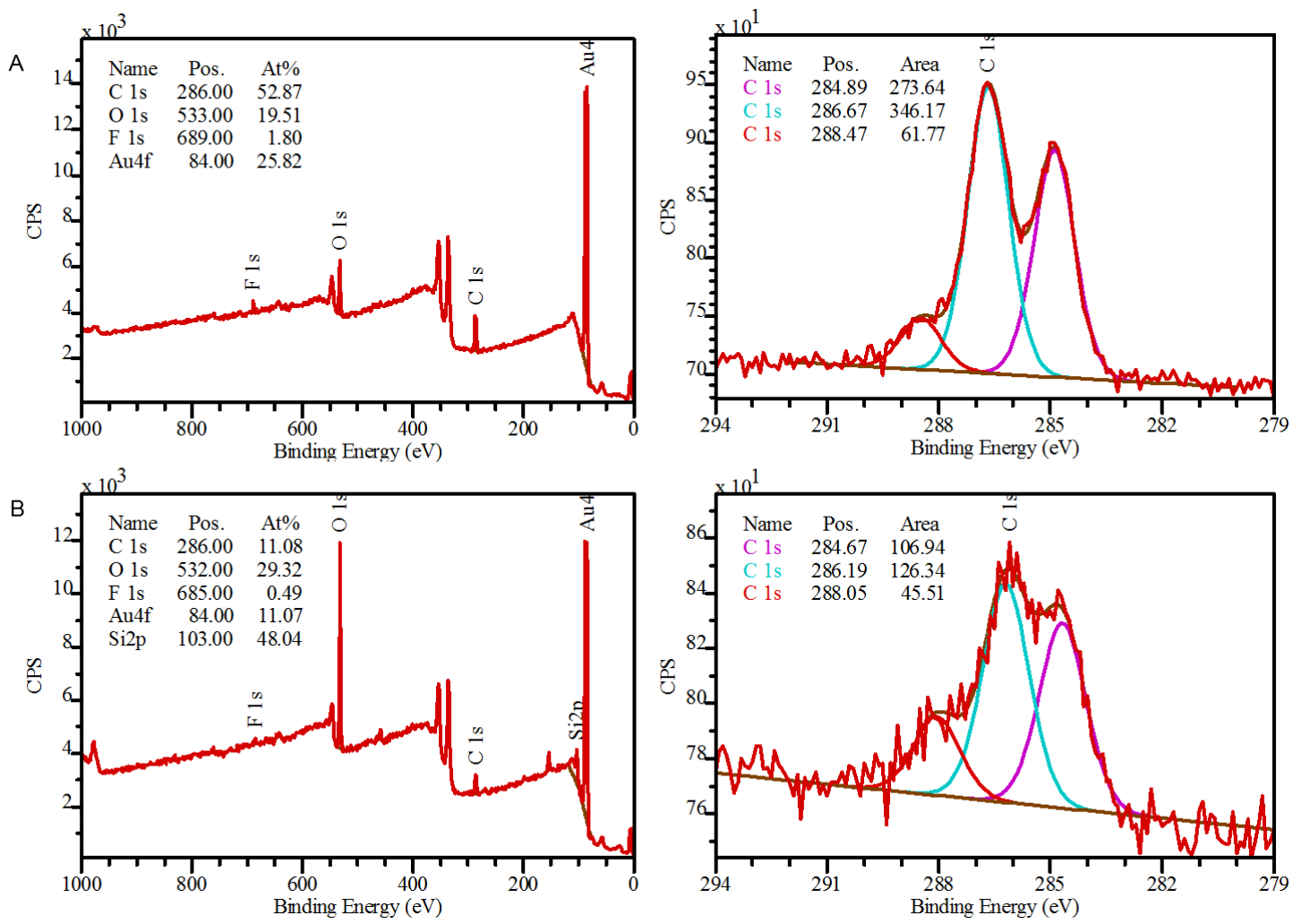

Figure 2. XPS survey spectra (left) and C1s narrow scan (right) of chips with a A) high contact angle $\left(60^{\circ}-90^{\circ}\right)$ and $\mathrm{B}$ ) low contact angle $\left(10^{\circ}-30^{\circ}\right)$ after modification with PEG3500. 


\section{Bio-functionalization of the antifouling layer}

Non-specific interactions are among the major challenges of any label-free biosensor and were therefore addressed in Chapter $\mathbf{2}$ of the thesis. In terms of antifouling coatings, chemistries well known from the surface modification of flat gold surfaces were used for the nanostructured biosensor chips. Similar to flat gold, excellent antifouling behavior following modification with both PEG and zwitterionic polymers was also observed for nanostructured gold. Another important criterion for these antifouling chemistries is their suitability for the immobilization of ligands. This turned out to be more challenging than expected from antifouling literature. Zwitterionic sulfobetaine methacrylate (SBMA) polymers, exhibiting optimal antifouling behaviour according to the literature and in our experiments, were chosen for further bio-functionalization. The protocols reported in the literature are based on functionalization of the bromide group. ${ }^{1,2}$ The first approach, starting with conversion of the bromide to amine followed by attachment of bis-NHS seemed quite straightforward (Figure $3 \mathrm{~A}$ ). However, this reaction is very difficult to monitor using conventional surface analysis techniques such as XPS, mainly because no unique atom is introduced in the modification steps. Furthermore, the activation with bisNHS is sensitive to water, making the analysis even more challenging. Therefore our assessment of the reaction was only based on the final results of biosensing. As demonstrated in the literature, ${ }^{2}$ the method works for detecting the binding of streptavidin to a biotinylated surface, using fluorescence. However, even after several modifications of the reported protocol, the indirect mycotoxin assay using OVA conjugates could not be successfully performed. This could be either due to the differences in sensitivities of the two techniques or due to the limited amount of OVA conjugates immobilized on the surface. This was also observed in a recent study where the same biotin-streptavidin interaction could not be seen using reflectometry. ${ }^{3}$ The second approach using conversion of the bromide to an azide (Figure 3B) allows immobilization using a click reaction with a bicyclononyne (BCN) probe. ${ }^{1}$ This approach requires either modification of OVA conjugates with $\mathrm{BCN}$ or an extra step where a $\mathrm{BCN}$ NHS is used for amine coupling with the conjugates thus complicating the entire procedure. In both approaches, the bromide group from the initiator that is required for the growth of the polymer is used. However the fact that it is available only in a limited amount is a constraint for further bio-functionalization. Furthermore, no success was achieved using the hydroxyl groups of the alternative PEGMA (Figure 3C) as suggested in the literature. ${ }^{4}$ Therefore, conventional PEG3500 with a carboxylic acid was chosen for the proof-of-principle experiments as well as for development of 6-plex mycotoxin assay in barley as described in Chapter 3. The activation of carboxylic acid groups using NHS/EDC allowed immobilization of larger molecules such as ovalbumin conjugates of mycotoxins via the amine groups (Figure 6A). In Chapter 4, carboxymethylated dextran 
(CMD) was chosen for direct immobilization of the toxins on the surface as CMD is a 3D hydrogel and provides more functional groups than 2D PEG. The CMD modification also helped to improve the stability of the chip by making them more resistant to scratching. Although several 2D and 3D chemistries have become commercially available, ${ }^{5} \mathrm{CMD}$ has dominated the SPR literature and continues to do so due to its wide applicability and high performance.
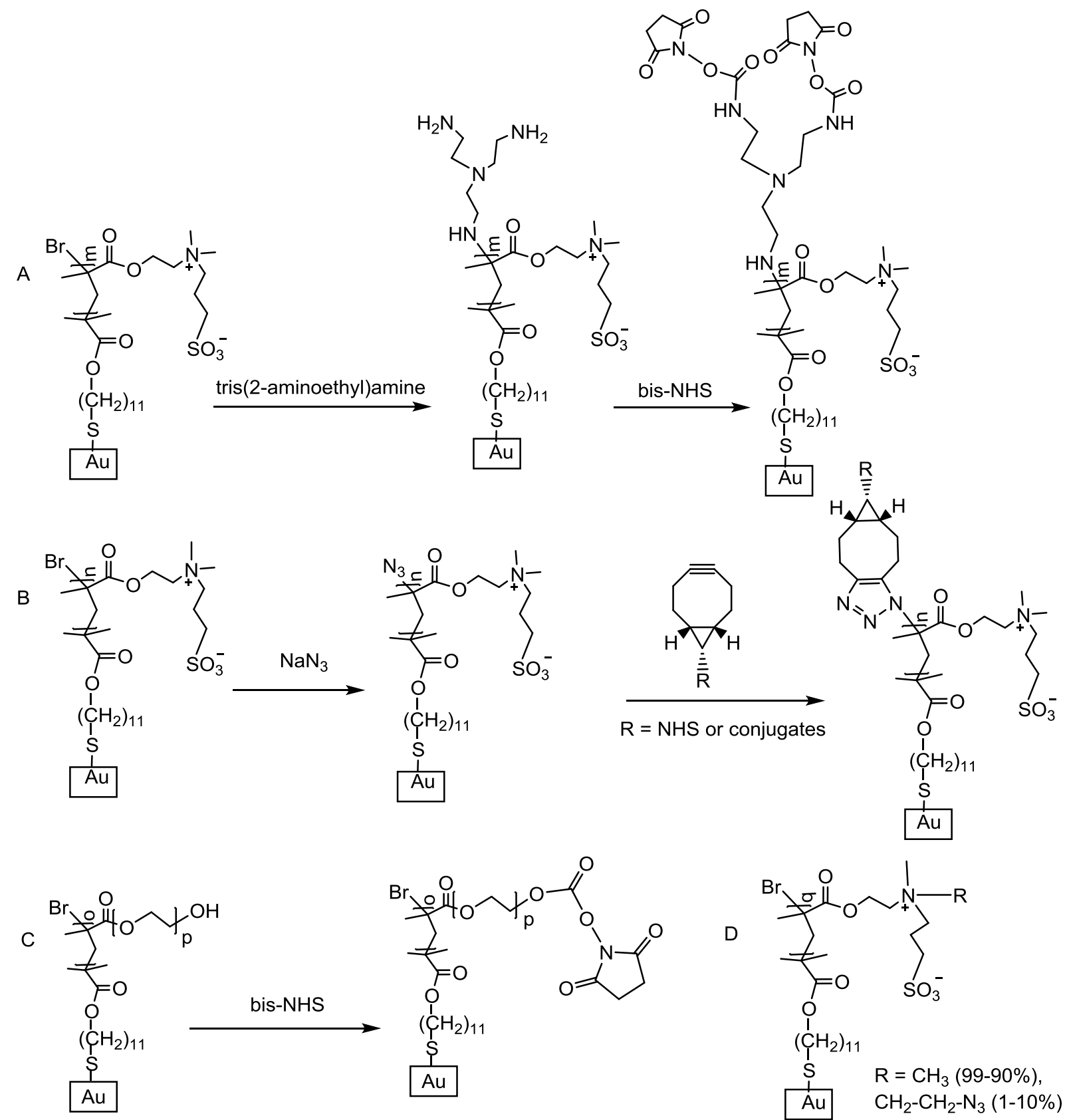

Figure 3. Different reported antifouling chemistries along with the strategies for their biofunctionalization. 


\section{Direct mycotoxin assay}

Due to the low molecular weight of mycotoxins, they do not give sufficient SPR signal upon binding to antibodies (in a direct assay) on the surface (Figure 4A). As a result, indirect assays are typically used in most applications. However, in this project the direct assay was also considered especially to study the effect of oriented antibody immobilization on the sensitivity of the assay. There is a lot of literature about different approaches for oriented antibody immobilization. ${ }^{6-9}$ Among these methods, use of protein $\mathrm{A} / \mathrm{G}^{10-12}$ and boronic acid ${ }^{13,14}$ are preferred approaches as they do not require modification of antibodies. However, the problem with both approaches is that the immobilization of antibodies is not covalent and therefore sensitive to regeneration, making them only suitable for single-use biosensor chips. There are several other methods for oriented immobilization of antibodies, often involving modification of antibodies. Most of them rely on attachment of a specific functional group (biotin, DNA, peptide) that would aid in the orientation of the antibodies. ${ }^{15-18}$ Additional modification of the antibodies complicated the protocol compared to the earlier two approaches.

The antibody against at least one of the toxins (DON) was successfully immobilized using a random amine coupling. A commercial ovalbumin conjugate of DON (DON-OVA) was first used as the competing probe and signal enhancer. However, a very low signal was obtained even at high concentrations (up to $100 \mu \mathrm{g} / \mathrm{mL}$ ) of the conjugate. Upon analyzing the solution using ESI-MS, free DON was observed. After purifying the DONOVA reagent using a $30 \mathrm{kDa}$ cut-off filter, the real-time biosensor signals were sufficient (Figure 4A) to construct a calibration curve (Figure 4B). The antibodies were randomly oriented on the surface and the chip could be used for several cycles with regeneration by a combination of $5 \mathrm{mM} \mathrm{HCl}$ and $5 \mathrm{mM} \mathrm{NaOH}$. Further research could not be performed within the timeframe of the thesis, but it would still be relevant to explore oriented antibody immobilization.

\section{Multiplex myctoxin assay}

Sample preparation still remains a bottleneck for multiplex mycotoxin detection. Firstly, the different chemical nature of the mycotoxins makes it challenging to find a "one size fits all" approach. The wide range of sensitivity (mainly influenced by the antibodyconjugate pairing) in combination with the range of legal limits complicates the process even more. For OTA and $\mathrm{AFB}_{1}$, the assay sensitivity needed to meet the maximum limits (MLs) set by the EU for unprocessed barley could not be achieved in Chapter 4 . In case of OTA, a trace enrichment step as performed in Chapter 5 helped to overcome the problem but of course complicated the sample preparation. While better antibodies might be a way to solve the problem, non-antibody based techniques ${ }^{19}$ such as those based on liposomes, ${ }^{20}$ aptamers $^{21}$ or peptides ${ }^{22}$ might also be explored. Aptamer-based SPR 
sensors are even more promising as aptamers for several mycotoxins have already been developed in the past years. ${ }^{23}$

Regeneration is one of the advantages of SPR biosensor assays over other immunoassays, since it reduces the (bio)reagent costs for at least one of the binding partners. However, it is also one of the challenges in multiplex assay development, especially when using protein conjugates of the analytes, as some of these conjugates are more labile than others. In the case of the mycotoxins studied here, the DON, T2 and $\mathrm{AFB}_{1}$ assays were the most critical ones with respect to regeneration: DON-OVA and T2OVA were very sensitive to regeneration whereas the antibodies against $\mathrm{AFB}_{1}$ were difficult to remove from the chip surface during regeneration. Following evaluation of a range of regeneration solutions, a combination of $10 \mathrm{mM} \mathrm{HCl}$ and $20 \mathrm{mM} \mathrm{NaOH}$ was found to be the best compromise. Furthermore, in the case of conventional SPR experiments using the Biacore where automated experiments can be performed, a decrease in the regeneration efficiency was observed over time. Upon further investigation, it was observed that when used in small volumes $(<1.5 \mathrm{~mL})$, the $\mathrm{pH}$ of the $\mathrm{NaOH}$ decreased over time. This is most likely due to the reaction of sodium hydroxide with carbon dioxide to form sodium carbonate, ${ }^{24}$ which results in a significant $\mathrm{pH}$ decrease in case of small volumes and subsequently decreases regeneration efficiency.

A

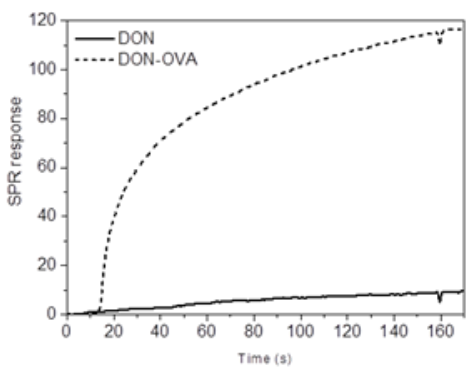

B

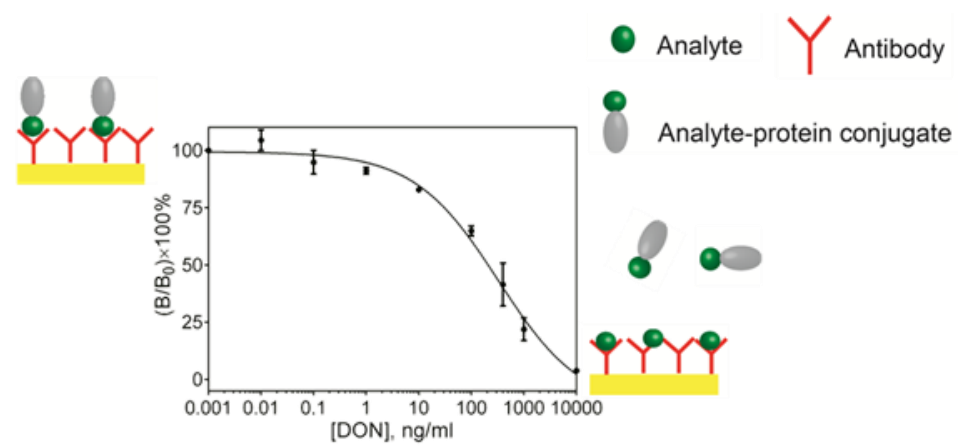

Figure 4. A) Signal obtained using a direct assay format for DON and DON-OVA. B) Calibration curve obtained for DON using a direct assay. The measurements were performed on a carboxymethylated dextran modified flat gold chip having antibodies against DON in a conventional Biacore instrument.

\section{Second generation instrument}

A new version of the instrument (Figure 5) has been recently developed consisting of modular fluidics, electronics and optics components. These can be easily combined and connected to any electronic component (laptop, tablet or smartphone) as long as the dedicated software is available. This gives the flexibility to choose whether or not to incorporate an auto-sampler as well as allowing easy maintenance of the instrument. 
Furthermore, the same flow-cell as well as the chip from the first generation instrument can be used thus simplifying transition to the second generation. However, at least in this very first new prototype version, the instrument appears bigger and more complicated due to the multiple separate components and their connections. On the bright side, the two LEDs ( $850 \mathrm{~nm}$ and $940 \mathrm{~nm}$ ) can now be used in combination, thereby somewhat improving the signal to noise ratio. With increasing refractive index, the intensity of the reflected light decreases between 750-850 nm, whereas it increases above $900 \mathrm{~nm}$. Therefore, use of the two LEDs in alternation and combining the two signals allows improvement in sensitivity by increasing the total signal. During the initial tests the signal obtained for antibodies improved such that only $5 \mu \mathrm{g} / \mathrm{mL}$ anti-DON/anti-OTA gave a similar response as $10 \mu \mathrm{g} / \mathrm{mL}$ of anti-DON/anti-OTA using one LED (850 $\mathrm{nm}$ ) in the first generation instrument. Furthermore, the updated software allows the spatial resolution to be chosen without compromising the time resolution. This can be especially important in high-throughput applications where high spatial resolution is required.

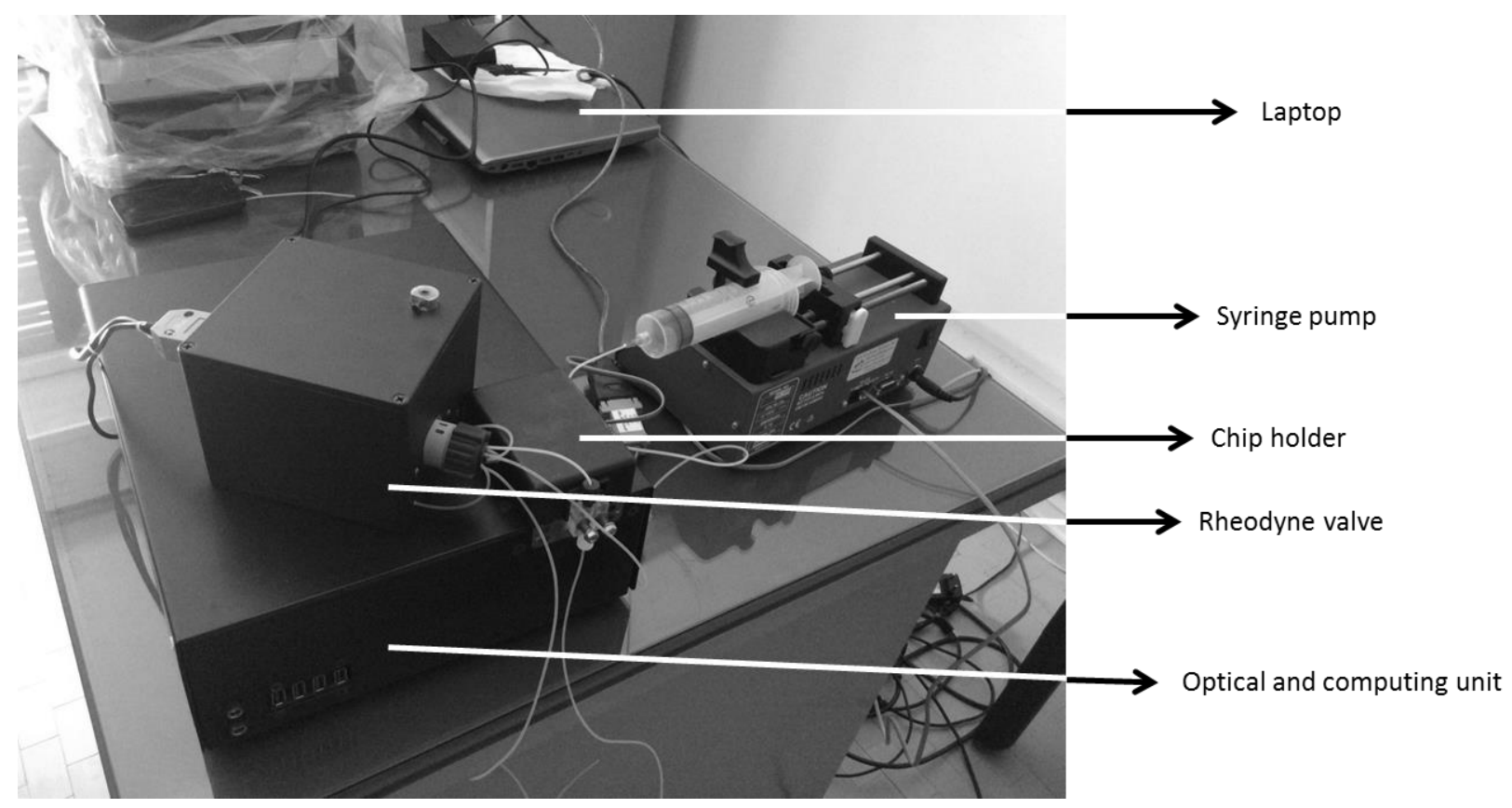

Figure 5. Picture of the first prototype of the second generation nanoplasmonics instrument.

\section{Coupling of SPR and MS}

The biochip spray described in Chapter 5 allows direct and simplified coupling of SPR with ambient MS. It is a powerful technique as it combines the selectivity of SPR biosensors with chemical identification of MS. In addition to DON, the method could be expanded to other mycotoxins and even other analytes. Furthermore, there are a few challenges that need to be overcome before the technique can be used for routine analysis. Cost of the analysis is one of the major challenges. This is mainly due to the 
easy deactivation of the antibodies, which makes the chip only suitable for single use. The cost issue was partially overcome by using four corners of the chip. In terms of cost reduction, one possible solution would be to use instruments with higher spatial resolution that would allow analysis of several interactions on a single chip. Development of either other antibodies that are reusable or non-antibody based recognition elements such as peptides might contribute to creating stable biosensor chips. Moreover, the analysis was performed manually and is less suitable for screening of multiple samples. Use of high-resolution mass spectrometry and therefore analysis of multiple analytes on the same chip would also contribute positively to the ease of analysis aspect as each analysis only takes a few seconds. These instruments could also allow automatic measurements on different parts of the chip thus reducing the need for manual operation.

\section{Future perspectives}

In addition to the research described in this thesis, there is still future research that can be performed to further develop the nanostructured iSPR instrument as well as to improve the mycotoxin assay. Some suggestions are given below.

\section{Antifouling chemistry}

In terms of antifouling chemistries, mixed zwitterionic polymers with small percentages (even as little as $1-10 \%$ ) of functional monomers seem to be promising. ${ }^{3}$ They combine the excellent antifouling properties of the traditional zwitterionic polymers with high efficiency of bio-functionalization as each branch of the functional polymer contains an azido group (Figure 3D). As demonstrated by the authors in a proof-of-principle experiment, a bicyclononyne ( $\mathrm{BCN}$ ) probe could be used for bio-functionalization. A similar approach using an azido poly-(L-lysine)-graft-poly(ethylene glycol) (APP) could be another option. ${ }^{25}$ Increasing the availability of various probes with $\mathrm{BCN}^{26}$ could help to expand the application of this chemistry. A recently reported antifouling zwitterionic peptide molecule 22 has been shown to be especially effective for site-specific immobilization of proteins.

\section{Signal enhancement}

Utilization of confinement induced enhancement of SPR signal ${ }^{27-29}$ would make the technology superior to other (i)SPR technologies. To achieve this effect, as discussed earlier (Page 138 and 139), reproducible chips with accurate control over the height of the PMMA wells is essential. ${ }^{30}$ Furthermore, one of the most popular approaches for molecular signal enhancement suggested in the literature is via the use of gold 
nanoparticles. ${ }^{31,32}$ Both electrostatic ${ }^{33,34}$ and covalent ${ }^{35}$ modification of gold nanoparticles with proteins has been suggested in the literature. However, the attachment of DON-OVA to gold nanoparticles did not show any enhancement in the SPR assay. On the other hand, the covalent approach proved experimentally difficult as aggregation of the gold nanoparticles was observed when NHS/EDC was used to activate the carboxylic acid groups on the nanoparticle surface. Based on the knowledge gathered during direct immobilization of mycotoxins, covalent attachment of mycotoxins directly on gold nanoparticles might be a feasible approach towards signal enhancement.

\section{Direct immobilization of toxins}

As demonstrated in Chapter 4, direct immobilization of DON and OTA resulted in higher sensitivity as well as far superior chip durability. However, due to the limited amount of functional groups present, direct immobilization of mycotoxins still remains a major challenge. For a surface containing carboxylic acid groups (Figure 6, iv) T2 and ZEA could, similar to DON, be directly immobilized on the surface using the hydroxyl group. The hydroxyl groups can be activated using carbonyldiimidazole (CDI) (Figure 6, ii) followed by addition to a surface containing amine groups (Figure 6, vi). On the other hand, $\mathrm{FB}_{1}$ is much more flexible with three different functional groups $(\mathrm{COOH}, \mathrm{OH}$ and $\mathrm{NH}_{2}$ ). The $\mathrm{COOH}$ groups of $\mathrm{FB}_{1}$ can be activated using EDC/NHS (Figure $6, \mathrm{i}$ ) as done for OTA while the $\mathrm{NH}_{2}$ groups can be attached directly on a NHS activated surface (Figure 6, v). Aflatoxin is even more challenging due to the lack of an easily modifiable functional group thus requiring an additional linker. One of the reactions used in the literature ${ }^{36}$ converts the carbonyl group into a carboxylic acid using O-(carboxymethyl) hydroxylamine (Figure $6 \mathrm{~B}$ ) so that the same strategy as OTA (Figure 6, i) can be used. The choice of strategy would be influenced by information about the binding sites of the antibodies as well as sensitivity of the functional groups. 
A

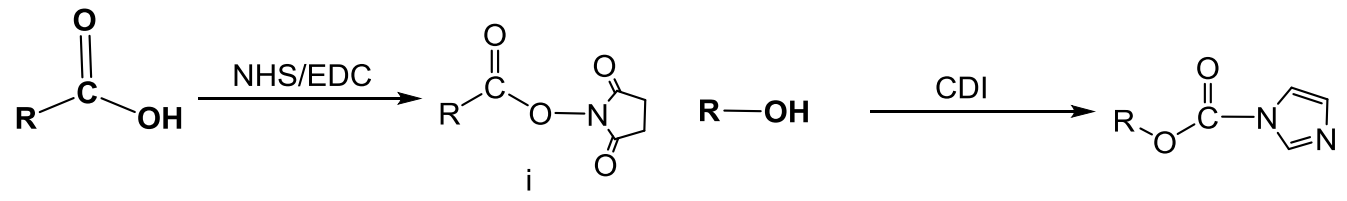

ii

B<smiles>COc1cc2c(c3c1CCC3=O)C1=CC=CC1OC(=O)C2=O</smiles><smiles>COc1cc2c(c3c1CCC3=NOCC(=O)O)C1=C(CCC1)OC(=O)O2</smiles>

iii

C
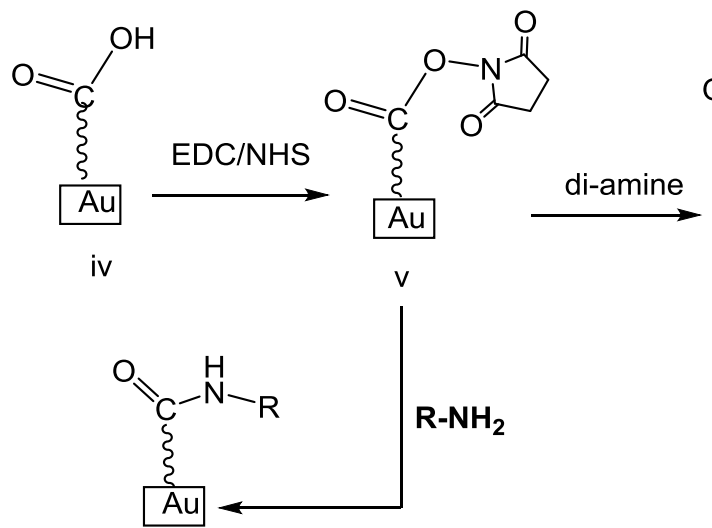<smiles>[R]C(=O)NCCOCCNC(=O)C1CCC1</smiles>

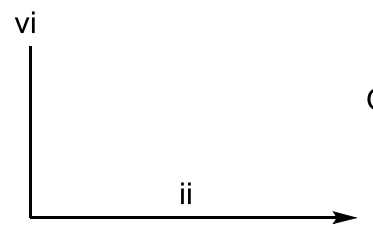<smiles>[R]OC(=O)NCCOCCNC(=O)C1CC1</smiles>

Figure 6. A) Activation of carboxylic acid and hydroxyl groups and $B$ ) modification of aflatoxin $B_{1}$ to $\mathrm{AFB}_{1}$-(O-carboxymethyl) oxime derivative before being immobilized on the surface. C) Chemical strategies for direct immobilization of mycotoxins with different functional groups on a surface containing carboxymethylated groups.

\section{Practical implications}

The CMD modification of the nanostructured iSPR chips were attempted using the protocols reported in the literature ${ }^{37,38}$ (Figure 7) but with no success. Therefore, custom modification was performed by Xantec (Düsseldorf, Germany). This adds additional cost and should be addressed in the future in view of the potential of valorization of the technology.

The immobilization of toxins/conjugates on the nanostructured gold chips performed in this thesis was done manually on the bench. A contact printer (continuous flow microspotter; Wastach Microfluidics, USA), used in the initial phase of the project, suffered from the drawback of requiring large volumes $(80 \mu \mathrm{L})$ compared to $1-2 \mu \mathrm{L}$ in the manual approach. A Scienion S3 (Scienion AG, Berlin, Germany) non-contact printer helped to overcome the problem of volume consumption, but was not suitable for hydrophilic surfaces (such as CMD) as the solution was prone to spreading. Therefore, use of a contact printer that allows spotting of toxins/conjugates using low volumes 
would offer a suitable compromise. This is an essential step towards making the technology suitable for kit suppliers. A chip with toxins would be part of such a kit.

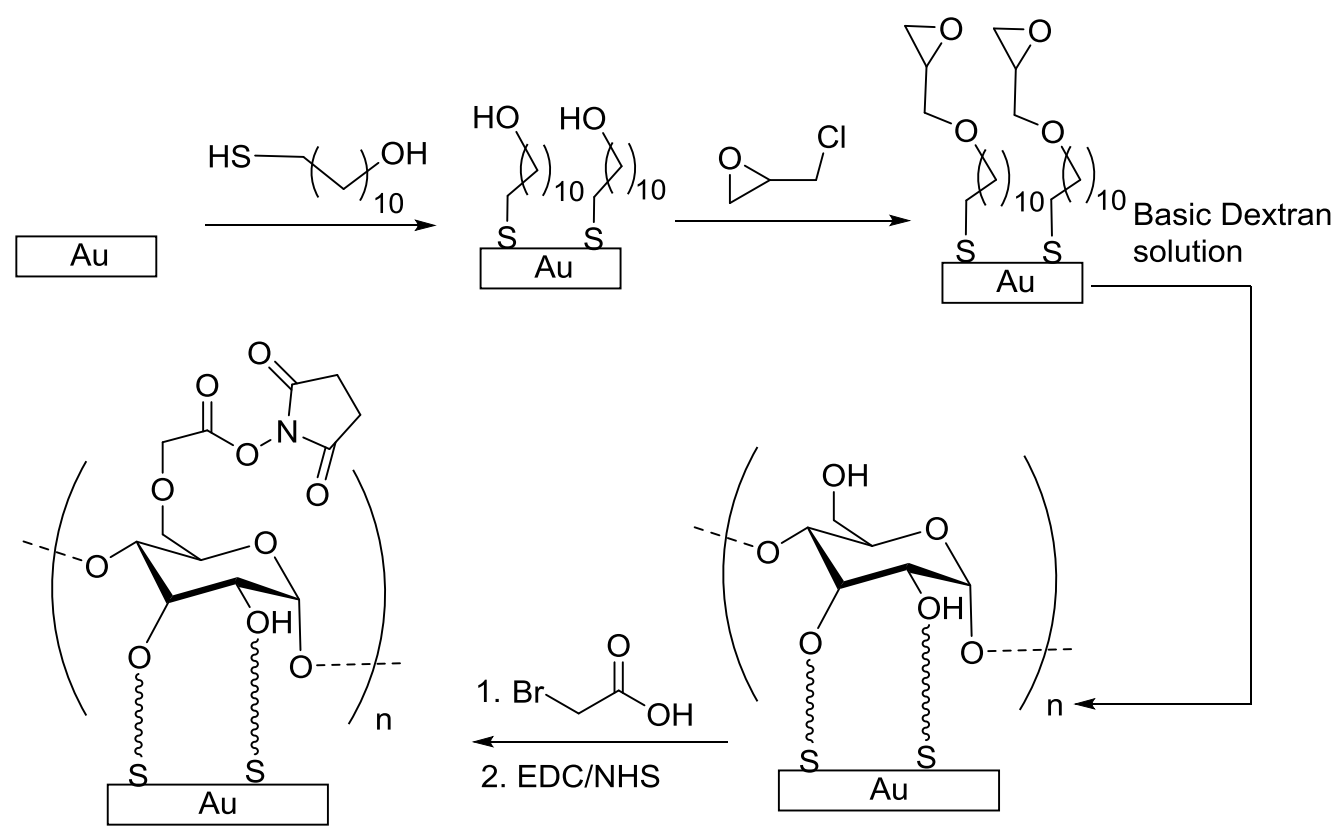

Figure 7. One of the chemical routes based on the literature ${ }^{37,38}$ used for modification of iSPR chips with carboxymethylated dextran (CMD).

\section{Optimization of hardware and software}

The current iSPR set-up only allows a single flow-cell. Therefore, if two toxins require different sample preparation they need to be injected separately thus increasing the analysis time. Keeping in mind the range in legal limits, sample preparation, and sensitivity of the immobilized analytes towards regeneration, having access to at least two or three independent flow-cells with iSPR capabilities would be advantageous. Of course, this would require considerable changes in the fluidics if all the channels were to be used at the same time, but the potential might be worth the investments required to achieve this.

At the beginning of this project, a portable instrument attached to a laptop computer was technically advanced. However, in recent years technology is moving towards mobile phone or tablet platforms to make a truly handheld device. The current setup only allows manual injection of the analytes and requires user attention during the run. Therefore, automation is an integral step towards future wider use of this instrument. This, of course, would not be compatible with the handheld format of the instrument, but would be more suitable for at-line applications where an additional automation unit can be connected to the handheld device. Automation would greatly benefit the DON assay as only mixing of sample with antibodies is required and many samples could be analyzed within a few hours. This concept change has already been partially addressed in the new version of the instrument where the start of the injection 
has to be done manually but the stop of the injection is done by the software based on the defined flow rate and injection volume. In terms of data, the current software generates an .xls file after the experiment consisting of the data required for generating a sensorgram (average intensity for each ROI for each time point). Automated data analysis will help to make the technique usable even for less-trained personnel. Furthermore, the current software does not include any tools for kinetic data analysis. Information about real-time kinetics is crucial in evaluating performance of new antibody-antigen pairs or in finding information about the binding sites. In the time span of the project, several SPR instruments have already been developed and the technology is moving towards smartphone-based SPR. ${ }^{39,40}$ This might also make the technology more appealing for the field of biodiagnostics. ${ }^{41}$ One of such devices reported in the literature $^{39}$ (Figure 8 ) uses a red image displayed on the screen as a light source and the front camera as the detector. A separate miniaturized optical coupling unit consisting of a prism, installed on top of the screen, allows attachment of a gold chip to the optics. Finally a microfluidic cell is added to the top to allow analysis of different samples.

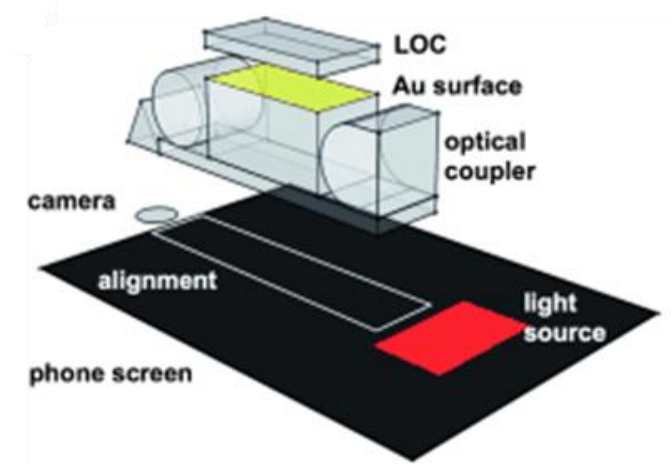

Figure 8. Schematic representation of a mobile phone based SPR biosensor (reproduced with permission from ref. 39).

\section{Conclusions}

In conclusion, this thesis provides the outcomes of the first steps towards the development of a nanostructured iSPR instrument for real-life food applications. Extensive optical and chemical characterization of the nanostructured iSPR chips was performed along with modification using well-known antifouling chemistries. A multiplex assay for in-field or at-line detection of myctoxins in beer and barley was successfully developed. Whenever relevant, a comparison was made with conventional (i)SPR instruments and with established reference methods such as LC-MS/MS, and these proved the substantial potential of the portable iSPR. Finally, the coupling of SPR with ambient mass spectrometry was developed, to add to the biosensing abilities of SPR, and to enable rapid identification of any SPR-observed analytes. 


\section{References}

1. Li, Y.; Giesbers, M.; Gerth, M.; Zuilhof, H. Generic top-functionalization of patterned antifouling zwitterionic polymers on indium tin oxide. Langmuir 2012, 28, 12509-12517.

2. Nguyen, A. T.; Baggerman, J.; Paulusse, J. M. J.; Zuilhof, H.; van Rijn, C. J. M. Bioconjugation of protein-repellent zwitterionic polymer brushes grafted from silicon nitride. Langmuir 2012, 28, 604-610.

3. Lange, S.; van Andel, E.; Smulders, M.; Zuilhof, H. Efficient and tunable 3D functionalization of fully zwitterionic antifouling surface coatings. Langmuir 2016, 32, 10199-10205.

4. Lee, B. S.; Chi, Y. S.; Lee, K.-B.; Kim, Y.-G.; Choi, I. S. Functionalization of poly(oligo(ethylene glycol) methacrylate) films on gold and $\mathrm{Si} / \mathrm{SiO} 2$ for immobilization of proteins and cells: SPR and QCM studies. Biomacromolecules 2007, 8, 3922-3929.

5. SPR sensorchips. http://www.xantec.com/products/spr_sensorchips.php (Accessed on: 19 August 2016).

6. Kausaite-Minkstimiene, A.; Ramanaviciene, A.; Kirlyte, J.; Ramanavicius, A. Comparative study of random and oriented antibody immobilization techniques on the binding capacity of immunosensor. Anal. Chem. 2010, 82, 6401-6408.

7. Vashist, S. K.; Dixit, C. K.; MacCraith, B. D.; O'Kennedy, R. Effect of antibody immobilization strategies on the analytical performance of a surface plasmon resonance-based immunoassay. Analyst 2011, 136, 4431-4436.

8. Makaraviciute, A.; Ramanaviciene, A. Site-directed antibody immobilization techniques for immunosensors. Biosens. Bioelectron. 2013, 50,460-471.

9. Trilling, A. K.; Beekwilder, J.; Zuilhof, H. Antibody orientation on biosensor surfaces: A minireview. Analyst 2013, 138, 1619-1627.

10. Lin, P.-C.; Chen, S.-H.; Wang, K.-Y.; Chen, M.-L.; Adak, A. K.; Hwu, J.-R. R.; Chen, Y.-J.; Lin, C.-C. Fabrication of oriented antibody-conjugated magnetic nanoprobes and their immunoaffinity application. Anal. Chem. 2009, 81, 8774-8782.

11. Ryu, Y.; Jin, Z.; Kang, M.; Kim, H.-S. Increase in the detection sensitivity of a lateral flow assay for a cardiac marker by oriented immobilization of antibody. BioChip J. 2011, 5, 193-198.

12. Shen, G.; Cai, C.; Wang, K.; Lu, J. Improvement of antibody immobilization using hyperbranched polymer and protein A. Anal. Biochem. 2011, 409, 22-27.

13. Ho, J.-a. A.; Hsu, W.-L.; Liao, W.-C.; Chiu, J.-K.; Chen, M.-L.; Chang, H.-C.; Li, C.-C. Ultrasensitive electrochemical detection of biotin using electrically addressable site-oriented antibody immobilization approach via aminophenyl boronic acid. Biosens. Bioelectron. 2010, 26, 1021-1027.

14. Duval, F.; van Beek, T. A.; Zuilhof, H. Key steps towards the oriented immobilization of antibodies using boronic acids. Analyst 2015, 140, 6467-6472.

15. Cho, I.-H.; Paek, E.-H.; Lee, H.; Kang, J. Y.; Kim, T. S.; Paek, S.-H. Site-directed biotinylation of antibodies for controlled immobilization on solid surfaces. Anal. Biochem. 2007, 365, 14-23.

16. Seo, M.-H.; Han, J.; Jin, Z.; Lee, D.-W.; Park, H.-S.; Kim, H.-S. Controlled and oriented immobilization of protein by site-specific incorporation of unnatural amino acid. Anal. Chem. 2011, 83, 2841-2845.

17. Seymour, E.; Daaboul, G. G.; Zhang, X.; Scherr, S. M.; Ünlü, N. L.; Connor, J. H.; Ünlü, M. S. DNAdirected antibody immobilization for enhanced detection of single viral pathogens. Anal. Chem. 2015, $87,10505-10512$.

18. Kruis, I. C.; Lowik, D. W. P. M.; Boelens, W. C.; van Hest, J. C. M.; Pruijn, G. J. M. An integrated, peptide-based approach to site-specific protein immobilization for detection of biomolecular interactions. Analyst 2016, 141, 5321-5328.

19. Chen, H.; Huang, J.; Palaniappan, A.; Wang, Y.; Liedberg, B.; Platt, M.; Tok, A. I. A review on electronic bio-sensing approaches based on non-antibody recognition elements. Analyst 2016, 141, 2335-2346.

20. Oh, E. H.; Lee, S. H.; Ko, H. J.; Park, T. H. Odorant detection using liposome containing olfactory receptor in the SPR system. Sens. Actuat. B: Chem. 2014, 198, 188-193.

21. Zhu, Z.; Feng, M.; Zuo, L.; Zhu, Z.; Wang, F.; Chen, L.; Li, J.; Shan, G.; Luo, S.-Z. An aptamer based surface plasmon resonance biosensor for the detection of ochratoxin $A$ in wine and peanut oil. Biosens. Bioelectron. 2015, 65, 320-326.

22. Dudak, F. C.; Boyaci, İ. H. Peptide-based surface plasmon resonance biosensor for detection of staphylococcal enterotoxin B. Food Anal. Methods 2014, 7, 506-511.

23. Ruscito, A.; Smith, M.; Goudreau, D. N.; DeRosa, M. C. Current status and future prospects for aptamerbased mycotoxin detection. J. AOAC Int. 2016, 99, 865-877.

24. Yoo, M.; Han, S.-J.; Wee, J.-H. Carbon dioxide capture capacity of sodium hydroxide aqueous solution. J. Environ. Manage. 2013, 114, 512-519.

25. van Dongen, S. F. M.; Janvore, J.; van Berkel, S. S.; Marie, E.; Piel, M.; Tribet, C. Reactive proteinrepellent surfaces for the straightforward attachment of small molecules up to whole cells. Chem. Sci. 2012, 3, 3000-3006.

26. Synaffix Reagents, BCN reagents. http://synaffix.biedmeer.nl/Webwinkel-Category-1277450/BCNAdaptable-probes.html (Accessed on: 18 August 2016).

27. Shalabney, A.; Abdulhalim, I. Sensitivity-enhancement methods for surface plasmon sensors. Laser Photon. Rev. 2011, 5, 571-606.

28. Breault-Turcot, J.; Masson, J.-F. Nanostructured substrates for portable and miniature SPR biosensors. Anal. Bioanal. Chem. 2012, 403, 1477-1484.

29. Ertsgaard, C. T.; McKoskey, R. M.; Rich, I. S.; Lindquist, N. C. Dynamic placement of plasmonic hotspots for super-resolution surface-enhanced raman scattering. ACS Nano 2014, 8, 10941-10946.

30. Valsesia, A.; Marabelli, F.; Giudicatti, S.; Marchesini, G. R.; Rossi, F.; Colpo, P. SPR sensor device with nanostructure. World Patent 2013, WO 2013/007448 A1. 
31. Hong, X.; Hall, E. A. H. Contribution of gold nanoparticles to the signal amplification in surface plasmon resonance. Analyst 2012, 137, 4712-4719.

32. Springer, T.; Ermini, M. L.; Spackova, B.; Jablonku, J.; Homola, J. Enhancing sensitivity of surface plasmon resonance biosensors by functionalized gold nanoparticles: Size matters. Anal. Chem. 2014, 86, 10350-10356.

33. Moon, J.; Kim, G.; Lee, S. A gold nanoparticle and aflatoxin B1-BSA conjugates based lateral flow assay method for the analysis of aflatoxin B1. Materials 2012, 5, 634-643.

34. Wang, X.; Niessner, R.; Knopp, D. Magnetic bead-based colorimetric immunoassay for aflatoxin B1 using gold nanoparticles. Sensors 2014, 14, 21535-21548.

35. Li, D.; He, Q.; Cui, Y.; Duan, L.; Li, J. Immobilization of glucose oxidase onto gold nanoparticles with enhanced thermostability. Biochem. Biophys. Res. Comm. 2007, 355, 488-493.

36. Khademi, F.; Mohammadi, M.; Kiani, A.; Haji Hosseini Baghdadabadi, R.; Parvaneh, S.; Mostafaie, A. Efficient conjugation of aflatoxin M1 with bovine serum albumin through aflatoxin M1-(o-carboxymethyl) oxime and production of anti-aflatoxin M1 antibodies. Jundishapur J. Microbiol. 2015, 8, 168501168507.

37. Schartner, J.; Hoeck, N.; Guldenhaupt, J.; Mavarani, L.; Nabers, A.; Gerwert, K.; Kotting, C. Chemical functionalization of germanium with dextran brushes for immobilization of proteins revealed by attenuated total reflection fourier transform infrared difference spectroscopy. Anal. Chem. 2015, 87, 7467-7475.

38. Löfås, S.; Johnsson, B. A novel hydrogel matrix on gold surfaces in surface plasmon resonance sensors for fast and efficient covalent immobilization of ligands. J. Chem. Soc., Chem. Comm. 1990, 1526-1528.

39. Preechaburana, P.; Gonzalez, M. C.; Suska, A.; Filippini, D. Surface plasmon resonance chemical sensing on cell phones. Angew. Chem. Int. Ed. 2012, 51, 11585-11588.

40. Liu, Y.; Liu, Q.; Chen, S.; Cheng, F.; Wang, H.; Peng, W. Surface plasmon resonance biosensor based on smart phone platforms. Sci. Rep. 2015, 5, 128641-128649.

41. Howes, P. D.; Rana, S.; Stevens, M. M. Plasmonic nanomaterials for biodiagnostics. Chem. Soc. Rev. 2014, 43, 3835-3853. 



\section{Summary}

The testing and further development of a prototype nanostructured imaging surface plasmon resonance (iSPR) biosensor, with a focus on surface modification and detailed characterization of the biosensor chip and in-field and at-line applicability in the food industry is described. Furthermore, a simplified coupling of SPR and MS is described that allows identification of the mycotoxins of interest along with any other cross-reacting analytes. Chapter 1 describes general information about SPR, SPR instruments along with their components, development of a multiplex SPR biosensor and coupling of SPR to mass spectrometry.

In Chapter 2, the surface modification, in-depth characterization and the antifouling performance of the nanostructured iSPR chip is described. Different types of polyethylene glycol (PEG) and zwitterionic polymers were chosen as antifouling chemistries. Various surface characterization techniques such as atomic force microscopy, scanning electron microscopy, water contact angle, X-ray photoelectron spectroscopy and direct analysis in real time high resolution mass spectrometry provided complementary information about the chip before and after the modification. Antifouling chemistry, an essential first step in the development of an SPR biosensor, prevents false positive results arising from non-specific binding of sample components to the SPR chip. Upon comparison of the surface modification and antifouling behavior with conventional flat SPR chips, the latter were only slightly better. Zwitterionic polymers and long chain PEG had the best antifouling performance. A proof-of-principle experiment was done to demonstrate the selective detection of streptavidin binding to a surface partially modified with biotin.

A 6-plex SPR assay for the detection of mycotoxins in barley was developed in Chapter 3. A benchmark double 3-plex assay was developed for the detection of deoxynivalenol (DON), zearalenone (ZEA), T-2 toxin (T-2), ochratoxin A (OTA), fumonisin $B_{1}\left(F B_{1}\right)$ and aflatoxin $B_{1}\left(A F B_{1}\right)$ using benchtop SPR instrument (Biacore). Preliminary in-house validation of the competitive inhibition assay developed using ovalbumin conjugates of the mycotoxins showed that the method is suitable for detection of DON, ZEA, T-2 and $\mathrm{FB}_{1}$ whereas further improvement is required for OTA and $A F B_{1}$. The method was then transferred to the nanostructured iSPR, which although less sensitive than the benchtop SPR, was able to detect DON, T-2, ZEA and $\mathrm{FB}_{1}$ at the relevant levels.

In Chapter 4, the assay developed in Chapter $\mathbf{3}$ was further optimized and an entire assay along with in-house validation and measurement of naturally contaminated was developed using the nanostructured iSPR. The antifouling chemistry used in Chapter 3, PEG, was replaced by carboxymethylated dextran (CMD) that not only allowed direct 
immobilization of toxins but also helped to improve the stability of the chip whereby the chip could be used for more than 450 cycles. DON could be detected at the relevant levels in beer with minimal sample preparation whereas for OTA an enrichment step using solid phase extraction was required.

As demonstrated in Chapter $\mathbf{3}$ and $\mathbf{4}$, the nanostructured iSPR instrument can be used for screening of different mycotoxins in beer and related ingredients. However, SPR is not able to provide chemical information of the binding analyte especially in cases where the antibodies have cross-reactivity towards conjugates of the analyte. Therefore, a simplified coupling for SPR with ambient mass spectrometry was developed in Chapter 5. The method allowed identification of DON as well as its cross-reacting conjugates such as deoxynivalenol-3-glucoside and 3-acetyl DON.

The research presented in this thesis is an important step towards the use of the nanostructured iSPR instrument for label free in-field and at-line detection of various analytes. In Chapter 6, discussion of the main achievements of this thesis, challenges and future perspectives of the technology is described. 


\section{Acknowledgements}

I would like to start by thanking my three supervisors Michel Nielen, Teris van Beek and Han Zuilhof for their help and support during the last four years. Michel and Teris thanks to your fast responses, I was always able to make my deadlines, even during the busy times of September teaching. Michel, thank you for helping me to come back on track when I got lost trying to do everything at once. Teris, I will never forget your help with the experiments during my last months that set the first steps for Chapter 5. Also, thank you for showing me a little bit of the Netherlands as part of the day trips you organized and for all the get together events we had at your house. Han, thank you for helping with all the surface chemistry related problems and for always finding time in your busy schedule to read my manuscripts.

I would also like to thank all my colleagues at ORC, Ton, Maurice, Tom, Cees, Maarten, Floris, Bauke, Elly, Aleida, Anita, Barend, Anne-Marie, Cees, Carel, Erik, Hendra, Marcel, Ronald, Remco, Elbert, Pepijn, Frank, Judith, Willem, Alexandre, Anke, Jaime, Sidhu, Umesh, Radostina, Saurabh, Nagendra, Satesh, Yao, Steven, Wilco, Florine, Aline, Bas, Kuldeep, Txema, Bram, Frank, Jorin, Tjerk, Rickdeb, Christie, Wang, Fatima, Sjoerd, Medea, Esther, Digvijay, Fred, Stefanie, Jorge, Rui, Jorick, Milou and Pepijn for creating a nice working environment. Barend, Pepijn, Ton, Maarten, Bas and Esther, it was great fun to supervise practicals with all of you. Txema thank you for your help in the lab and additionally for modifying some surfaces for me. Thank you Elbert for your help with the fluidics of my instrument, I learned a lot from it. And Frank thank you for being there when I jumped from the MS room to your office asking for help. Tjerk, Digvijay and Medea, it was great organizing the PhD trip and of course getting to know a little bit more about all the PhDs during these trips. Wilco thank you for being such a nice office mate and being there throughout the PhD journey. Thank you for answering all the questions and helping me during the entire thesis submission process. Jorin, I will miss our dinner and board game nights but hope that we can still find time to see each other. Aline and Fatima, it was a lot of fun to go for Zumba classes; without you guys I am sure I would have missed quite a few of them. Esther Roeven and Esther van Andel for accepting to be my paranymphs and I wish you all the best for your own PhD. Thank you to Surfix and Aquamarijn team for being part of all the fun activities both inside and outside the lab.

Thanks to my colleagues at RIKILT, Susann, Ana, Jeroen and Nathalie for helping me get around in your lab, if I first managed to get past the reception of course. Willem Haasnoot and Jeroen Peters thank you for all the discussions and ideas during the project. Jeroen also thank you for the collaboration and providing naturally contaminated beer samples. 
I would like to thank all my project partners for the discussions and feedback. Jeroen Kool, Dina Lakayan and Dick Iperen, thank you for your help in designing and making the flow cell. I would like to thank the Netherlands Organization for Scientific Research (NWO) and COAST for the financial support and the project partners of VU Amsterdam, RIKILT, Heineken, Synthon, Technex, EuroProxima, Waterproef and associated partners Plasmore and Bionavis for stimulating discussions during the project meetings. I would also like to additionally thank Europroxima, Heineken, Plasmore, RIKILT and VU for hosting me during the secondments.

I am grateful to my students Laura Schijven, Anna Segarra Fas, Marloes van Adrichem and Rumaisha Annida for their hard work and wish them all the best for their future. All of your support has contributed to finalizing my thesis. Anna, I really appreciated your enthusiasm, which resulted in Chapter 3. Nida, thank you for all the hours you worked on the mycotoxin assay for beer, which made Chapter 4 possible.

I would also like to thank my former colleagues in Germany and Spain who have helped to pave the path until I decided to pursue a PhD degree. Patricia, I would not have survived 3 months in Spain without speaking a word of Spanish if it wasn't for you and your wonderful family. Special thanks to Prof. Dr Werner Nau without whose coaching I would not be the scientist that I am today and Dr Maik Jacob for being so supportive and believing in me during my stay in the Nau group as well as during my PhD application.

I would like to thank my Dutch teacher and classmates who made the learning process so much fun.

A big thank you to all my friends and my host family for making Bremen my second home. Amita di and Saksham dai thank you for coming to all my graduation ceremonies, you make me feel like my family is there. I would like to thank Abhinandan Shrestha for the friendship and academic career we shared and for going through all my ups and downs together in the past nine years. Alexandra thank you for being such a good friend and an awesome house mate, I miss you and our never ending conversations.

I would like to thank all my new colleagues at AkzoNobel for creating a nice working environment. Working extra hours during the evening did not feel so bad when we knew there would be cake the next day. Maaike thank you for thinking along for the design of my cover, it motivated me to get going with it.

Wouter, I am glad I chose to come to Wageningen for my PhD as there was something even more important I found here. Thank you for being so supportive and having dinner ready when I arrived home late, sometimes even five days a week. I would also like to thank your family, Marjan, Theo, Charlotte and Robin who have been like a family away from home. And of course little Noudje for bringing a smile to all our faces. 
Of course last but not the least my parents and my brother for their love and support. The highest credit goes to my mother, the inspiration and motivation of my life. You made the choice to rather skip my graduation and come to support me during the difficult last months when I was trying to manage a new house, new job and finalize my $\mathrm{PhD}$; this is just one of the many things you have done to help me fulfil my dreams. 



\section{Curriculum vitae}

Sweccha Joshi was born on 25 August 1987 in Kathmandu, Nepal. After finishing her high school in 2007, she moved to Germany to pursue a bachelor studies in Chemistry at Jacobs University Bremen. In 2010, she decided to continue at Jacobs University and obtained a Master of Science degree in Nanomolecular Science in 2012. During her bachelor and masters studies she conducted research in the group of Prof. Dr

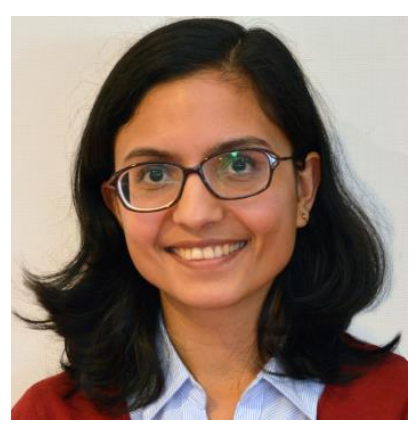

Werner Nau working on the study of interaction of metal oxide nanoparticles with short peptides and amino acids. In 2009, she spent three months in the lab of Prof. Dr Uwe Pischel as an intern working on the synthesis and photophysical characterization of a novel fluorescent probe for $\mathrm{pH}$ modulated switching of supramolecular host-guest complexes. In July 2012, Sweccha moved to the Netherlands to start a PhD project under the supervision of Prof. Dr Michel Nielen, Prof. Dr Han Zuilhof and Dr Teris van Beek, the results of which are shown in this thesis. Since July 2016, she works as a researcher in the team of Dr Michel Rosso at the Decorative Coatings business unit of AkzoNobel in Sassenheim. 



\section{List of publications}

1. Joshi, S.; Ghosh, I.; Pokhrel, S.; Mädler, L.; Nau, W. M., Interactions of amino acids and polypeptides with metal oxide nanoparticles probed by fluorescent indicator adsorption and displacement. ACS Nano 2012, 6, 5668-5679.

2. Manova, R. K.; Joshi, S.; Debrassi, A.; Bhairamadgi, N. S.; Roeven, E.; Gagnon, J.; Tahir, M. N.; Claassen, F. W.; Scheres, L. M. W.; Wennekes, T.; Schroën, K.; van Beek, T. A.; Zuilhof, H.; Nielen, M. W. F., Ambient surface analysis of organic monolayers using direct analysis in real time orbitrap mass spectrometry. Analytical Chemistry 2014, 86, 2403-2411.

3. Joshi, S.; Pellacani, P.; van Beek, T. A.; Zuilhof, H.; Nielen, M. W. F., Surface characterization and antifouling properties of nanostructured gold chips for imaging surface plasmon resonance biosensing. Sensors and Actuators $B$ : Chemical 2015, 209, 505-514.

4. Joshi, S.; Segarra-Fas, A.; Peters, J.; Zuilhof, H.; van Beek, T. A.; Nielen, M. W. $F$, Multiplex surface plasmon resonance biosensing and its transferability towards imaging nanoplasmonics for detection of mycotoxins in barley. Analyst 2016, $141,1307-1318$.

5. Joshi, S.; Annida, R.; Zuilhof, H.; van Beek, T. A.; Nielen, M. W. F, Analysis of mycotoxins in beer using a portable nanostructured imaging surface plasmon resonance biosensor. Journal of Agricultural and Food Chemistry 2016, 64, 82638271.

6. Joshi, S.; Zuilhof, H.; van Beek, T. A.; Nielen, M. W. F, Biochip spray: Simplified coupling of surface plasmon resonance biosensing and mass spectrometry. Analytical Chemistry 2017, doi:10.1021/acs.analchem.6b04012. 



\section{Overview of completed training activities}

\section{Discipline specific activities}

Advanced Organic Chemistry (VLAG), Wageningen, the Netherlands, 2012-2014

Advanced Food Analysis (VLAG), Wageningen, the Netherlands, 2013

NWO Study Group Analytical Chemistry (NWO), Lunteren, the Netherlands, 2012-2013

TA-COAST Programme Meeting (NWO, TI-COAST), Lunteren/Amersfoort, the Netherlands, 2012-2016

Advances in Biodetection and Biosensors (Selectbio), Berlin, Germany, 2014

CHAINS (NWO), Veldhoven, the Netherlands, 2014-2015

Micronano Conference (MinacNed), Amsterdam, the Netherlands, 2015

$26^{\text {th }}$ Anniversary World Congress on Biosensors (Elsevier), Gothenburg, Sweden, 2016

\section{General courses}

VLAG PhD Week (VLAG), Baarlo, the Netherlands, 2012

Techniques for Writing and Presenting (WGS), Wageningen, the Netherlands, 2013

Project and Time Management (WGS), Wageningen, the Netherlands, 2013

ACS on Campus (ACS), Utrecht, the Netherlands, 2013

Mini-symposium: How to Write a World Class Paper (WUR Library), Wageningen, the Netherlands, 2013

Guide to Scientific Artwork (WUR Library), Wageningen, the Netherlands, 2015

Career Perspectives (WGS), Wageningen, the Netherlands, 2015

\section{Optionals}

Preparation of PhD Research Proposal, 2012

Colloquia (ORC), 2012-2016

Project Meetings, 2012-2016

Group Meetings (ORC), 2012-2016

PhD trip (ORC) to Germany and Switzerland, 2013

PhD trip (ORC) to Canada, 2015

Organizing committee PhD trip (ORC) to Canada, 2015 
The research presented in this thesis was financially supported by The Netherlands Organization for Scientific Research (NWO) in the framework of the Technology Area COAST (project nr 053.21.107) with WU, VU Amsterdam, RIKILT, Heineken, Synthon, Technex, EuroProxima, Waterproef as partners and Plasmore and Bionavis as associated partners.

Financial support from Wageningen University for printing this thesis is gratefully acknowledged.

Cover design: Sweccha Joshi

Printed by Digiforce, Proefschriftmaken.nl, Wageningen, the Netherlands 
